\title{
Gastrointestinal Tract and Abdomen
}

\section{ESOPHAGUS}

\section{STOMACH}

G-1 Retention of Barium in the Hypopharynx

(Esp. Cricopharyngeal Achalasia) (Roentgen Counterpart of Dysphagia)

G-2 Esophageal Motility Disorder (Aperistalsis, Tertiary

Contractions, Spasm, and other Forms)

G-3 Achalasia (Cardiospasm) of the Esophagus

G-4 Air in the Esophagus

G-5

G-6

G-7

G-8

G-9

G-10-1

G-10-2

G-10-3

G-10-4

G-11

G-12

G-13

G-14

G-15

G-16

G-17

G-18

G-19

G-20
Double-Barrel Esophagus

Diverticulum of the Esophagus-Upper Third

Diverticulum of the Esophagus-Middle Third

Diverticulum of the Esophagus-Lower Third

Diverticula of the Esophagus-Diffuse

Solitary Intramural or Intraluminal Filling Defect of the Esophagus

Localized Constriction or Narrowing of the Esophagus

Thickening of the Esophageal Wall (CT, US, MRI)

Esophageal Ulceration(s)

Transverse Mucosal Folds in the Esophagus

Widespread Irregular or Nodular Esophageal Mucosa

Esophageal Plaques

Esophageal Varices

Esophageal Lesion in a Child 
Filling Defect(s) in the Stomach (Intraluminal, Mucosal, or Intramural)

G-22 Filling Defect in a Gastric Remnant Lesion Involving the Gastric Fundus (Intrinsic or Extrinsic) Gastric Ulceration

\section{Erosive Gastritis}

Large Gastric Folds (Local or Widespread); Also Thickening of the Stomach Wall on CT, US, or MRI

G-27 Linitis Plastica Pattern of the Stomach (See G-28)

G-28 Narrowing or Deformity of the Gastric Antrum (See G-27)

Combined Gastric Antral and Duodenal Disease Gastric Outlet Obstruction Dilatation of the Stomach without Obstruction Interstitial Emphysema of the Stomach Gastrocolic or Gastroduodenocolic Fistula Increased Retrogastric or Retroduodenal Space

\section{DUODENUM}

Diminished or Absent Fold Pattern in the Duodenum and Small Bowel

Nodular or Thickened Folds in the Duodenum; Also Thickening of the Duodenal Wall on CT, US, or MRI Postbulbar Duodenal Ulceration

\section{G-46 G-47 \\ G-48 \\ G-49-S1 \\ G-49-S2 \\ G-50}

G-51-1

G-51-2
Abnormal Position of Small Bowel Loops

Separation or Displacement of Small Bowel Loops

Solitary Mass in the Small Bowel with Preserved Mucosa

Benign Tumors of the Small Bowel

Malignant Tumors of the Small Bowel

Multiple Intraluminal, Mucosal, or Intramural Filling

Defects in the Small Bowel

Small Bowel Diverticulum

Small Bowel Pseudodiverticulum 
G-52 Thickening of the Small Bowel Wall, Generalized or Localized (Barium, US, CT, MRI)

G-53 Mucosal Destruction of the Small Bowel with or without Stricture (Local or Widespread)

G-54 Regular Thickening of Small Bowel Folds (>3 mm)

G-55 Generalized Irregular or Distorted Small Bowel Folds

G-56 Simultaneous Fold Thickening of the Stomach and Small Bowel

G-57 Malabsorption Pattern in the Small Bowel

G-58 Blind Loop Syndrome

G-59 Small Bowel Dilatation with Thickened Mucosal Fold Pattern

G-60

G-61

Small Bowel Dilatation with Normal Fold Pattern

Acute Nonobstructive Small Bowel Distention

("Paralytic Ileus")

G-62 Chronic Nonobstructive Small Bowel Distention

G-63 Terminal Ileum Lesion

SMALL AND LARGE BOWEL

\begin{tabular}{|c|c|}
\hline G-64-S & Nondiaphragmatic Hernias \\
\hline G-65 & Abnormalities of Bowel Rotation \\
\hline G-66 & Congenital Syndromes Associated with Intestinal Malrotation \\
\hline G-67 & Aphthoid Ulcers in the Small Bowel or Colon \\
\hline G-68 & $\begin{array}{l}\text { Innumerable Tiny Nodules (Sand-like or Granular Lucencies } \\
\text { Smaller Than } 5 \mathrm{~mm} \text { ) in the Small Bowel or Colon }\end{array}$ \\
\hline-69 & $\begin{array}{l}\text { Mesenteric Vascular Compromise (Intestinal Ischemia, } \\
\text { Infarction) }\end{array}$ \\
\hline-70 & Gas in the Bowel Wall (Pneumatosis Intestinalis) \\
\hline G-71-1 & $\begin{array}{l}\text { Small and Large Bowel Wall Thickening: Homogeneous } \\
\text { (Stratified or Mixed) Attenuation on Postcontrast CT }\end{array}$ \\
\hline 71 ? & $\begin{array}{l}\text { Small and Large Bowel Wall Thickening: Heterogeneous } \\
\text { (Stratified or Mixed) Attenuation on Postcontrast CT }\end{array}$ \\
\hline G-72-1 & Mild Thickening $(<2 \mathrm{~cm})$ of Bowel Wall on Postcontrast CT \\
\hline G-72-2 & $\begin{array}{l}\text { Marked Thickening }(>2 \mathrm{~cm}) \text { of Bowel Wall on } \\
\text { Postcontrast CT }\end{array}$ \\
\hline G-73 & $\begin{array}{l}\text { Symmetric versus Asymmetric Thickening of Bowel Wall on } \\
\text { Postcontrast CT }\end{array}$ \\
\hline G-74-1 & Focal Bowel Wall Thickening $(<10 \mathrm{~cm})$ on Postcontrast CT \\
\hline G-74-2 & $\begin{array}{l}\text { Segmental Bowel Wall Thickening }(10-30 \mathrm{~cm}) \text { on } \\
\text { Postcontrast CT }\end{array}$ \\
\hline G-74-3 & Diffuse Bowel Wall Thickening on Postcontrast CT \\
\hline G-75 & $\begin{array}{l}\text { Residual Intestinal Barium After Gastrointestinal Study } \\
\text { (More Than One Week) }\end{array}$ \\
\hline 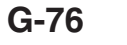 & Intestinal Obstruction in a Newborn \\
\hline G-77 & Intestinal Obstruction in a Child (See G-76) \\
\hline
\end{tabular}




\section{G-78 \\ G-79}

G-80

\section{APPENDIX, COLON, AND RECTUM} AND RECTUM
G-81

G-82-S

G-83

G-84-S

G-85

G-86

G-87

G-88

G-89

G-90

G-91

G-92

G-93

G-94

G-95-1

G-95-2

G-96

G-97

G-98

G-99

G-100

G-101

G-102

G-103

G-104

G-105

G-106

G-107

Intestinal Obstruction in an Adult

Intestinal Pseudo-Obstruction (Ogilvie Syndrome); Bowel Obstruction in the Absence of Mechanical Blockage Sentinel Loop (Localized Dilatation of Small and/or Large Bowel)

Appendiceal Lesion or Mass Adjacent to Appendix Congenital Anomalies and Variations of the Appendix Solitary Filling Defect in the Colon Classification of Colonic Tumors and Tumor-Like Lesions Annular ("Apple Core" or "Napkin Ring") Lesion of the Colon

Segmental Narrowing of the Colon

Multiple Filling Defects in the Colon

Colitis (Ulceration, Edema, Spasm); Also Thickening of the Colon Wall on CT, US, or MRI

Pericolic Abscess

Double Tracking of Barium in the Distal Colon

Smooth Colon

Colonic Distention without Obstruction

Megacolon

Toxic Megacolon

Colon of Reduced Caliber (Microcolon) in a Newborn

Meconium Plug in a Newborn

Cecal Lesion

Conical or Contracted Cecum

Enlargement of the Ileocecal Valve

Anterior Indentation on the Rectosigmoid Junction

Rectal Disease on Barium Enema

Rectovaginal Fistula

Increased Retrorectal or Presacral Space (See C-50) 

Biliary Obstruction Without Dilatation

G-133-1 Cystic and Saccular Lesions of the Bile Ducts-With Normal-sized Intrahepatic Bile Ducts (CAG, MRCP)

G-133-2 Cystic and Saccular Lesions of the Bile Ducts-With Dilatation of Intrahepatic Bile Ducts (CAG, MRCP)

G-134 Filling Defect or Segmental Lesion in the Bile Ducts (CAG, MRCP, US, CT)

G-135 Thickening of Bile Duct Walls (US)

G-136 Echoes Within the Bile Ducts (US)

G-137 Bile Duct Narrowing or Obstruction (CAG, MRCP) 
G-138 Enlarged Papilla of Vater

G-139 Biliary-Enteric Fistula (See G-128)

G-140 Biliary-Pleural (or Bronchial) Fistula

\section{LIVER}

DIFFUSE OR MULTIFOCAL LIVER DISEASE

$\begin{array}{ll}\text { G-141-1 } & \text { Hepatomegaly (See G-141-2) } \\ \text { G-141-2 } & \begin{array}{l}\text { Congenital Hepatomegaly } \\ \text { G-142 }\end{array} \\ \text { Diffuse Hepatic Calcifications (PF, US, CT) } \\ \text { G-143 } & \text { Fetal or Neonatal Liver Calcification } \\ \text { G-144 } & \begin{array}{l}\text { Fatty Change in the Liver (Steatosis) (US, CT, MRI) } \\ \text { G-145 }\end{array} \\ \text { Perfusion Abnormalities of the Liver } \\ \text { Patchy Hepatogram (Areas of Low Density on Angiography } \\ \text { G-147 } & \begin{array}{l}\text { or Postcontrast CT) } \\ \text { Generalized or Multifocal Decreased Echogenicity of the } \\ \text { Liver on Ultrasound (Hypoechoic) }\end{array} \\ \text { G-148 } & \begin{array}{l}\text { Generalized or Multifocal Increased Echogenicity of the } \\ \text { Liver on Ultrasound (Hyperechoic) } \\ \text { Generalized High Density Liver (Nonenhanced CT) }\end{array} \\ \text { G-149 } & \begin{array}{l}\text { Generalized or Multifocal Low Density Liver } \\ \text { (Nonenhanced CT) }\end{array} \\ \text { G-151 } & \begin{array}{l}\text { Multiple Hypointense Liver Lesions on T2-Weighted MR } \\ \text { Images }\end{array} \\ \text { G-152 } & \text { Diffusely Decreased Liver Intensity on MRI } \\ \text { G-153 } & \text { Spontaneous Liver Rupture }\end{array}$

FOCAL LIVER LESIONS

$\begin{array}{ll}\text { G-154 } & \text { Neoplasm of the Liver (Child or Adult) } \\ \text { G-155-1 } & \text { Solid Liver Lesion-Adult (US, CT, MRI) } \\ \text { G-155-2 } & \text { Solid Liver Lesion-Older Child or Adolescent } \\ \text { G-155-3 } & \text { Solid Liver Lesion_-Infant or Young Child (Under Age 5) } \\ \text { G-156 } & \text { Solid Liver Masses_-Multiple } \\ \text { G-157 } & \text { Cystic Liver Lesion(s) (US, CT, MRI) } \\ \text { G-158-1 } & \text { Liver Lesion Characterized by Linear or Stellate Central Scar } \\ & \text { (CT, US, Angio) } \\ \text { G-158-2 } & \text { Liver Lesion With Central Scar on MRI } \\ \text { G-159 } & \text { Liver Lesion Characterized by Blood or Hemorrhage } \\ \text { G-160 } & \text { Liver Lesion Characterized by Calcification } \\ \text { G-161 } & \text { Liver Lesion Characterized by Fat (CT, MRI) } \\ \text { G-162 } & \text { Liver Lesion Characterized by Bull's-Eye Appearance } \\ \text { G-163 } & \text { Liver Lesion Characterized by Fluid-fluid Level } \\ \text { G-164 } & \text { Liver Lesion Characterized by "Fill-in" (Angio, Postcontrast } \\ & \text { CT or MRI) } \\ \text { G-165-S } & \text { Liver Lesions-Clinical Considerations }\end{array}$




$\begin{array}{ll}\text { G-166 } & \text { Liver Metastases_Calcified } \\ \text { G-167 } & \text { Liver Metastases_Hypervascular } \\ \text { G-168 } & \text { Liver Metastases_Hemorrhagic } \\ \text { G-169 } & \text { Liver Metastases_Cystic } \\ \text { G-170 } & \text { Liver Metastases_-Ultrasound Characteristics } \\ \text { G-171 } & \text { Avascular or Hypovascular Liver Lesion (Angio, } \\ & \text { Postcontrast CT or MRI) } \\ \text { G-172 } & \text { Hypervascular or Hyperdense Liver Lesion (Angio, } \\ \text { G-173 } & \text { Postcontrast CT or MRI) } \\ \text { G-174 } & \text { Hypoechoic Liver Lesion (Usually Cystic on US) } \\ \text { G-175 } & \text { Hyperechoic Liver Lesion (US) } \\ \text { G-176 } & \text { Isoechoic Liver Lesion (US) } \\ \text { G-177 } & \text { Heterogeneous Hepatic Echogenicity (Parenchyma or Mass) } \\ & \text { on US } \\ \text { G-178-S } & \text { Hepatic Pseudolesion (US) } \\ \text { G-179 } & \text { Intrahepatic Acoustic Shadowing (Linear versus Focal) } \\ & \text { on US } \\ \text { G-180 } & \text { Focal Low Density (Decreased Attenuation) Liver Lesion } \\ & \text { (Nonenhanced CT) } \\ \text { G-181 } & \text { Focal Low Density Liver Lesion (Postcontrast CT) } \\ \text { G-182 } & \text { Focal High Density Liver Lesion (Nonenhanced CT) } \\ \text { G-184-S } & \text { MRI Characteristics of Various Liver Lesions } \\ \text { G-185 } & \text { Liver Lesions with Circumferential Rim on MRI } \\ \text { G-186 } & \text { Focal High Signal Intensity Liver Lesion on T1-Weighted } \\ & \text { MR Images }\end{array}$

HEPATIC AND PORTAL VEINS; PERIPORTAL AREA

\footnotetext{
G-187 Damping of Hepatic Vein Doppler Waveform (US)

G-188 Hepatic Vein Dilatation

G-189 Hepatic Vein Thromboembolism or Obstruction (BuddChiari S.) (Incl. Obstruction of Upper Inferior Vena Cava)

G-190 Portal Vein Thrombosis or Obstruction

G-191 Portal Hypertension

G-192 Gas in the Portal Veins

G-193 Periportal Hypoechogenicity on US/Periportal Low Density on CT

G-194 Prominent Periportal Echoes (Starry Sky Liver) in Adults on Ultrasound

G-195 Prominent Periportal Echoes in Neonates on Ultrasound

G-196 Increased Periportal Signal Intensity on MRI
} 
Splenomegaly: A Classification (See G-198)

G-199

Congenital Syndromes with Splenomegaly

G-200

Spenomegaly with Normal Echogenicity (US)

G-201

G-202

G-203

G-204

G-205

G-206

Splenomegaly with Diffuse Hypoechoic Pattern (US)

G-207

G-208

G-209

G-210

G-211

Splenomegaly with Diffuse Hyperechoic Pattern (US)

Small or Nonvisualized Spleen

Splenic Calcification-Solitary

Splenic Calcifications-Multiple

Solitary Lesion of the Spleen (US, CT, MRI)

Focal Cystic or Low Density Splenic Lesion (CT, MRIUsually Hypoechoic on US)

Focal Hyperechoic Splenic Lesion (US)

Diffuse Increased Splenic Density on CT

Multiple Splenic Hypointensities on MRI

G-212

PANCREAS

G-213

G-214

G-215 Complex Pancreatic Lesion (US)

G-216 Increased Echogenicity of Pancreas (US)

G-217 Focal Shadowing Pancreatic Lesions (US)

G-218 Pancreatic Calcification Without Mass

G-219 Pancreatic Lesion Characterized by Calcification

G-220 Pancreatic Lesion Characterized by Blood or Hemorrhage

G-221 Pancreatic Lesion Characterized by Fat

G-222 Fatty Replacement or Infiltration of Pancreas

G-223 Hypervascular Pancreatic Lesion (Postcontrast CT, MRI, or Angio)

G-224 Pancreatic Angiographic Abnormality (Displacement of Vessels, Encasement, Extravasation, Hypervascularity, Hypovascularity, Other)

G-225 Dilated Pancreatic Duct

G-226 Pancreatic Duct Stricture (ERCP, MRCP)

G-227 Gas in Pancreatic Duct 
MESENTERY AND

PERITONEUM
G-228

G-229

G-230

G-231

G-232

G-233

G-234

G-235

\section{ABDOMINAL AIR,} GAS, OR ABSCESS

G-237
Cystic Mesenteric or Intraperitoneal Lesion (US, CT, MRI)

Solid Lesion of the Mesentery or Mesenteric Root (US, CT, MRI)

Alteration in Density of the Mesenteric Fat on CT ("Misty Mesentery")

Peritoneal Disease

Diffuse Peritoneal Thickening (US, CT, MRI)

Peritoneal Fluid Collection (Ascites) in an Adult (Esp. on US, CT, MRI)

Peritoneal Fluid (Ascites) in an Infant or Child

Chylous or Lymphatic Ascites

G-236 Spontaneous Pneumoperitoneum without Peritonitis

G-238

G-239

G-240

G-241

G-242

G-243

Pneumoperitoneum with Peritonitis

Perforated Hollow Viscus in an Infant

Abnormal Gas Collection in the Right Upper Quadrant

Abnormal Gas Collection in the Left Upper Quadrant

Large Abdominal Gas Pocket

Abdominal or Pelvic Abscess

Right Anterior Pararenal Space Abscess

(Esp. on US, CT, or MRI)

G-244-S Abscess Mimics on Abdominal US, CT, or MRI

G-245

G-246

\section{ABDOMINAL \\ CALCIFICATIONS}

G-247-1 Abdominal Calcification(s) in an Infant or Child (See G-143, G-203, 204, G-247-2)

G-247-2 Congenital Syndromes with Abdominal Calcifications

G-248 Nonvisceral Abdominal Calcification

G-249 Abdominal Calcifications that Layer in the Upright Position

G-250

G-251 Widespread Abdominal Calcifications

G-252 Abdominal Wall Calcification

\section{ABDOMEN, MISCELLANEOUS}


G-256 Lower Abdominal Mass in a Neonate or Child

G-257 Abdominal Lymphadenopathy (US, CT, MRI)

G-258 Fat Density in the Abdomen (CT, MRI)

G-259 "Pseudokidney" or "Bull's-Eye" Sign in the Abdomen (US)

G-260 Abnormal Abdominal Vessels on Angiography

G-261 Complications of AIDS in the Gastrointestinal Tract and Abdomen 


\section{Gamut G-1 \\ RETENTION OF BARIUM \\ IN THE HYPOPHARYNX (ESP. CRICOPHARYNGEAL ACHALASIA) (ROENTGEN COUNTERPART OF DYSPHAGIA)}

\section{COMMON}

1. Connective tissue disease (collagen vascular

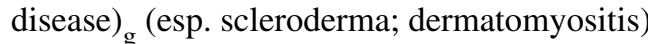

2. Cricopharyngeal achalasia (minor to severe muscular incoordination)

3. Esophageal obstruction (eg, achalasia; carcinoma)

4. Muscular disorder ${ }_{\mathrm{g}}$ (eg, myasthenia gravis; myotonic dystrophy; steroid or thyrotoxic myopathy; oculopharyngeal myopathy)

5. Neurologic disorder ${ }_{g}$ (eg, stroke; bulbar or pseudobulbar palsy; bulbar poliomyelitis; high unilateral cervical vagotomy; multiple sclerosis; parkinsonism; amyotrophic lateral sclerosis; syringomyelia; Riley-Day S. (familial dysautonomia); peripheral or central cranial nerve palsy; diphtheria; tetanus)

6. Postradiation therapy for pharyngeal or neck malignancy

7. Zenker's diverticulum

\section{UNCOMMON}

1. Abscess; cellulitis (esp. pharyngeal; peritonsillar)

2. Esophageal web (eg, congenital; Plummer-Vinson S.)

3. Foreign body

4. Hematoma of neck

5. Lymphadenopathy, cervical

6. [Pseudodefect from total laryngectomy]

7. Stricture (eg, lye)

8. Thyroid neoplasm

[ ] This condition does not actually cause the gamuted imaging finding, but can produce imaging changes that simulate it.

\section{References}

1. Eisenberg RL: Gastrointestinal Radiology: A Pattern Approach. (ed 3) Philadelphia: Lippincott, 1996, pp 5-8
2. Felson B: Chest Roentgenology. Philadelphia: WB Saunders, 1973

3. Jones B: Functional abnormalities of the pharynx. In: Gore RM, Levine MS: Textbook of Gastrointestinal Radiology. (ed 2) Philadelphia: WB Saunders, 2000, pp 316-328

4. Rubesin SE: Pharynx. In: Levine MS, Rubesin SE, Laufer I: Double Contrast Gastrointestinal Radiology. (ed 3) Philadelphia: WB Saunders, 2000, pp 61-91

\section{Gamut G-2}

\section{ESOPHAGEAL MOTILITY DISORDER (APERISTALSIS, TERTIARY CONTRACTIONS, SPASM, AND OTHER FORMS)}

\section{COMMON}

+*1. Achalasia (cardiospasm) (See G-3)

2. Chalasia (infantile gastroesophageal regurgitation)

3. Cricopharyngeal achalasia

+4 . Diffuse esophageal spasm, idiopathic

$+* 5$. Esophagitis (eg, reflux or peptic; radiation; caustic; monilial; herpes; viral)

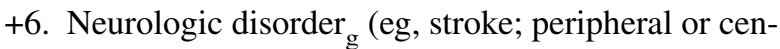
tral cranial nerve palsy; pseudobulbar palsy; bulbar poliomyelitis; syringomyelia; high unilateral cervical vagotomy; multiple sclerosis; parkinsonism; amyotrophic lateral sclerosis; cerebral disease; Riley-Day S. \{familial dysautonomia\})

+7. Obstructive lesion, extrinsic or intrinsic (eg, Schatzki ring; stricture; esophageal neoplasm; foreign body; web; mediastinal tumor, cyst, or lymphadenopathy; mediastinitis)

$+* 8$. Postsurgical repair of esophageal atresia, tracheoesophageal fistula, or hiatal hernia

+9. Presbyesophagus

*10. Scleroderma

11. Zenker's diverticulum

\section{UNCOMMON}

1. Alcoholic neuropathy

*2. Amyloidosis 
3. Behçet $\mathrm{S}$.

*4. Chagas' disease

5. Congenital syndromes (eg, Ehlers-Danlos S.; cutis laxa; G syndrome)

*6. Connective tissue disease (collagen vascular dis-

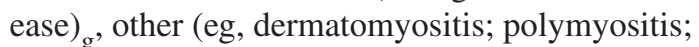
mixed connective tissue disease \{MCTD\}; lupus erythematosus; rheumatoid arthritis)

*7. Crohn's disease

+8 . Diabetic neuropathy

*9. Drug reaction (atropine; curare; Pro-Banthine)

+10 . Hypertensive lower esophageal sphincter

11. Iatrogenic (eg, postvagotomy S.; sclerotherapy for varices)

12. Intramural diverticulosis of esophagus

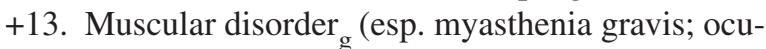
lopharyngeal myopathy; muscular or myotonic dystrophy; steroid or thyrotoxic myopathy)

+14. Neoplasm, infiltrative (eg, diffuse esophageal carcinoma; leukemia)

+15 . Nutcracker esophagus

16. Paraneoplastic S.

17. Thyroid disease (myxedema; thyrotoxicosis)

18. Tylosis (keratosis palmaris et plantaris familiaris)

* Esophagus may be aperistaltic.

+ Tertiary contractions often present.

\section{References}

1. Aly YA: Digital radiography in the evaluation of oesophageal motility disorders. Clin Radiol 2000;55:561-568

2. Bennett JR, Hendricks TR: Diffuse esophageal spasm: A disorder with more than one cause. Gastroenterology 1970; 59:273-279

3. Clouse RE, Diamant NE: Motor physiology and motor disorders of the esophagus. In: Feldman M, Scharschmidt BF, Sleisenger MH: Gastrointestinal and Liver Disease. (ed 6) Philadelphia: WB Saunders, 2000, pp 61-91

4. Eisenberg RL: Gastrointestinal Radiology: A Pattern Approach (ed 3). Philadelphia: Lippincott, 1996, pp 3-24

5. Gelfand DW: Gastrointestinal Radiology. New York: Churchill Livingstone, 1984, p 170

6. Hurwitz AL, Duranceau A, Haddad JK: Disorders of Esophageal Motility, vol 16. In: Smith LH Jr (ed): Major Problems in Internal Medicine series. Philadelphia: WB Saunders, 1979

7. Merhar G: Disorders of esophageal motility. Semin Roentgenol 1981;16:162-163
8. Ogle SJ, Kirk CJC, Bailey RJ, et al: Esophageal function in cirrhotic patients undergoing injection sclerotherapy for esophageal varices. Digestion 1978;18:178-185

9. Ott DJ: Esophageal motility disorders. Semin Roentgenol 1994:29:321-331

10. Ott DJ: Motility disorders of the esophagus. In: Gore RM, Levine MS: Textbook of Gastrointestinal Radiology. (ed 2) Philadelphia: WB Saunders, 2000, pp 316-328

11. Simeone JF, Burrell M, Toffler R, et al: Aperistalsis and esophagitis. Radiology 1977; 123:9-14

12. Teplick JG, Haskin NM: Roentgenologic Diagnosis. (ed. 3) Philadelphia: WB Saunders, 1976

13. Zboralske FF, Dodds WJ: Roentgenographic diagnosis of primary disorders of esophageal motility. Radiol Clin North Am 1969;7:147-162

\section{Gamut G-3}

\section{ACHALASIA (CARDIOSPASM) OF THE ESOPHAGUS}

\section{COMMON}

1. [Carcinoma, esophageal or gastric]

2. Idiopathic

3. [Stricture from esophagitis]

\section{UNCOMMON}

1. Amyloidosis

2. Cerebrovascular accident (stroke)

3. Chagas' disease

4. Chronic idiopathic intestinal pseudo-obstruction

5. Connective tissue disease (collagen disease) $)_{\mathrm{g}}$ (esp. scleroderma)

6. Diabetic neuropathy

7. Drug reaction (esp. atropine; Pro-Banthine)

8. Hypertensive lower esophageal sphincter

9. latrogenic (eg, vagotomy)

10. Nerve destruction (myenteric plexus, sympathetic, or vagus), esp. by neoplasm

[ ] This condition does not actually cause the gamuted imaging finding, but can produce imaging changes that simulate it. 


\section{References}

1. Eisenberg RL: Gastrointestinal Radiology: A Pattern Approach (ed 3). Philadelphia: Lippincott, 1996, pp 11-19

2. Gelfand DW: Gastrointestinal Radiology. New York: Churchill Livingstone, 1984, p 170

3. Ott DJ: Motility disorders of the esophagus. In: Gore RM, Levine MS: Textbook of Gastrointestinal Radiology. (ed 2) Philadelphia: WB Saunders, 2000, pp 316-328

4. Woodfield CA, Levine MS, Rubesin SE, et al: Diagnosis of primary versus secondary achalasia: reassessment of clinical and radiographic criteria. AJR 2000;175:727-731

\section{Gamut G-4}

\section{AIR IN THE ESOPHAGUS}

\section{COMMON}

1. Achalasia (See G-3)

2. Infant respiratory distress syndrome (hyaline membrane disease)

3. Normal

4. Respirator therapy; intubation

5. Scleroderma

\section{UNCOMMON}

1. Caustic esophagitis

2. Chagas' disease

3. Diverticulum, esophageal

4. Gastroesophageal regurgitation, infantile or adult

5. Hypertensive lower esophageal sphincter

6. Obstruction of esophagus (eg, neoplasm of esophagus; mediastinal tumor, cyst, or lymphadenopathy; mediastinitis)

7. Postoperative (eg, esophageal or pulmonary surgery)

\section{References}

1. Meredith HC, Anderson RJ, Vujic I: Another look at the segmental air esophagram. AJR 1960;136:23-26

2. Ott DJ: Motility disorders of the esophagus. In: Gore RM, Levine MS: Textbook of Gastrointestinal Radiology. (ed 2) Philadelphia: WB Saunders, 2000, pp 316-328

\section{Gamut G-5}

\section{EXTRINSIC IMPRESSION ON THE CERVICAL ESOPHAGUS}

\section{COMMON}

1. Cricopharyngeal muscle

2. [Esophageal web]

3. Lymph node enlargement

4. Osteophyte of cervical spine

5. Pharyngeal venous plexus (postcricoid impression)

6. Soft tissue abscess or hematoma (esp. retrolaryngeal)

7. Thyroid mass (eg, goiter; adenoma; carcinoma; thyroiditis)

\section{UNCOMMON}

1. Aneurysm or buckling of carotid or innominate artery

2. Cervical spine lesion, other (eg, osteomyelitis; neoplasm; anteriorly herniated disk)

3. Ectopic gastric mucosa

4. Parathyroid tumor (eg, adenoma; carcinoma)

5. [Gastrointestinal stromal tumor $_{\mathrm{g}}$ (esp. leiomyoma) or lipoma of esophagus]

6. Tracheal or laryngeal neoplasm

[ ] This condition does not actually cause the gamuted imaging finding, but can produce imaging changes that simulate it.

\section{References}

1. Gelfand DW: Gastrointestinal Radiology. New York: Churchill Livingstone, 1984

2. Eisenberg RL: Clinical Imaging: An Atlas of Differential Diagnosis. (ed 3) Philadelphia: Lippincott-Raven, 1997, pp 284-285 


\section{Gamut G-6 \\ EXTRINSIC IMPRESSION ON THE THORACIC ESOPHAGUS}

\section{COMMON}

1. Cardiac enlargement (esp. left atrium)

2. Duplication cyst (bronchogenic or enteric)

3. Hiatal hernia (esp. paraesophageal)

4. Mediastinal lymphadenopathy (eg, metastasis; lymphoma $_{\mathrm{g}}$; tuberculosis; histoplasmosis; sarcoidosis)

5. Mediastinal mass (eg, tumor; cyst; mediastinitis)

6. Normal structure (left main stem bronchus; aortic knob; confluence of left pulmonary veins)

7. Pericardial lesion (eg, effusion; cyst; tumor) (See E-43)

8. Pleuropulmonary fibrosis at lung apex, esp. tuberculosis (pseudoimpression)

9. Pulmonary mass (esp. carcinoma of lung)

10. Vascular impression, abnormal (eg, aortic aneurysm or tortuosity; coarctation of aorta; right, cervical, or double aortic arch; truncus arteriosus; aberrant right or left subclavian artery; pulmonary sling) (See G-7)

\section{UNCOMMON}

1. Neurinoma of vagus or phrenic nerve

2. Spinal abnormality (eg, kyphosis; scoliosis; osteophyte; DISH; neoplasm; osteomyelitis)

3. Tracheal neoplasm (See B-111, F-81-1)

\section{References}

1. Gelfand DW: Gastrointestinal Radiology. New York: Churchill Livingstone, 1984, p 190

2. Eisenberg RL: Clinical Imaging: An Atlas of Differential Diagnosis. (ed 3) Philadelphia: Lippincott-Raven, 1996, pp 286-291

\section{Gamut G-7}

\section{EXTRINSIC VASCULAR IMPRESSION ON THE ESOPHAGUS (See E-18)}

\section{COMMON}

1. Aberrant right or left subclavian artery

2. Aortic abnormality, acquired (eg, aneurysm; tortuosity)

3. Aortic knob

4. Coarctation of aorta

5. Right aortic arch (esp. posterior or type II aortic arch)

\section{UNCOMMON}

1. Anomalous innominate artery

2. Aortic diverticulum

3. APVC, total (below diaphragm)

4. Arteriovenous malformation

5. Azygos or hemiazygos vein dilatation

6. Cervical aortic arch

7. Corrected transposition (medially placed pulmonary artery)

8. Double aortic arch

9. Enlarged "bronchial" artery (incl. truncus arteriosus; absent main pulmonary artery)

10. Pulmonary artery "sling" (anomalous origin of left pulmonary artery)

11. Pulmonary vein confluence draining into back of left atrium

12. Sequestration of lung (anomalous artery from aorta)

\section{References}

1. Birnholz JC, Ferrucci JT, Wyman SM: Roentgen features of dysphagia aortica. Radiology 1974;111:93-99

2. Levine MS, Laufer I: Esophagus. In: Levine MS, Rubesin SE, Laufer I: Double Contrast Gastrointestinal Radiology. (ed 3) Philadelphia: WB Saunders, 2000, pp 90-125 


\section{Gamut G-8}

\section{TRACHEOESOPHAGEAL OR ESOPHAGOBRONCHIAL FISTULA}

\section{COMMON}

1. Carcinoma of esophagus, lung or trachea

2. Congenital, with or without esophageal atresia

\section{UNCOMMON}

1. Abscess, pulmonary or mediastinal

2. Actinomycosis

3. Behçet $\mathrm{S}$.

4. Caustic esophagitis (esp. lye)

5. Crohn's disease

6. Diverticulum of esophagus, perforated

7. Esophageal lung (sequestration)

8. Granulomatous lymphadenitis (esp. histoplasmosis; tuberculosis; syphilis)

9. Infected pulmonary or mediastinal cyst or sequestration of lung

10. Lymphoma

11. Radiation therapy

12. Rupture of esophagus, "spontaneous" or traumatic (eg, foreign body; missile), or iatrogenic (surgery; instrumentation)

\section{References}

1. Cameron DC: Non-malignant oesophago-bronchial fistulae in the adult: Case reports and review of the literature. Australas Radiol 1983; 27:143-153

2. DeBacker AI, De Schepper AM, Vaneerdeweg W: Esophagobronchial fistula following redo Nissen fundoplication. Abdom Imaging 2000;25:116-118

3. Eisenberg RL: Gastrointestinal Radiology: A Pattern Approach. (ed 3) Philadelphia: Lippincott, 1996, pp 131-139

4. Ramakantan R, Shah P: Tuberculous fistulas of the pharynx and esophagus. Gastrointest Radiol 1990;15:145-152

5. Spalding AR, Burney DP, Richie RE: Acquired benign bronchoesophageal fistulas in the adult. Ann Thorac Surg $1979 ; 28: 378-383$

\section{Gamut G-9}

\section{DOUBLE-BARREL ESOPHAGUS*}

\section{COMMON}

1. Dissecting intramural hematoma or hemorrhage

a. Severe vomiting (eg, Boerhaave S. with esophageal perforation, or Mallory-Weiss S. with esophageal tear)

b. Trauma

c. Instrumentation (eg, nasogastric intubation; endoscopy)

d. Ingestion of sharp foreign body

e. Spontaneous (eg, bleeding diathesis)

\section{UNCOMMON}

1. Esophageal duplication

2. Crohn's disease

3. Intraluminal diverticulum

4. Intramural abscess (eg, postendoscopy or foreign body perforation)

5. Intramural pseudodiverticulosis

* Barium opacification of an intramural dissecting channel separated from the normal esophageal lumen by an intervening radiolucent mucosal stripe.

\section{References}

1. Canon CL, Levine MS, Cherukuri R, et al: Intramural tracking: a feature of esophageal intramural pseudodiverticulosis. AJR 2000;175:371-374

2. Eisenberg RL: Gastrointestinal Radiology. A Pattern Approach. (ed 3) Philadelphia: Lippincott, 1996, pp 140-145

3. Ghahremani GG: Esophageal trauma. Semin Roentgenol 1994;24:387-400

4. Levine MS: Other esophagitides. In: Gore RM, Levine MS: Textbook of Gastrointestinal Radiology. (ed 2) Philadelphia: WB Saunders, 2000, pp 364-386 


\section{Gamut G-10-1}

\section{DIVERTICULUM OF THE ESOPHAGUS- UPPER THIRD}

\section{COMMON}

1. Zenker's diverticulum (pulsion type, posterior wall)

\section{UNCOMMON}

1. Killian-Jamieson diverticulum

2. Lateral pharyngeal pouch (pharyngocele), congenital or acquired (eg, glass blower; trumpet player)

3. Traction diverticulum (esp. after upper lobectomy, laryngectomy, or neck infection)

\section{References}

1. Eisenberg RL: Gastrointestinal Radiology: A Pattern Approach. (ed 3) Philadelphia: Lippincott, 1996, pp 119-124

2. Rubesin SE: Structural abnormalities of the pharynx. In: Gore RM, Levine MS: Textbook of Gastrointestinal Radiology. (ed 2) Philadelphia: WB Saunders, 2000, pp 227-255

\section{Gamut G-10-2}

\section{DIVERTICULUM OF THE ESOPHAGUS- MIDDLE THIRD}

\section{COMMON}

1. Acquired pulsion diverticulum due to esophageal dysmotility

2. Traction diverticulum (esp. from adherent granulomatous lymph node)

\section{UNCOMMON}

1. Congenital pulsion diverticulum (interaorticobronchial)

2. [Neurenteric or duplication cyst, communicating]

[ ] This condition does not actually cause the gamuted imaging finding, but can produce imaging changes that simulate it.

\section{References}

1. Eisenberg RL: Gastrointestinal Radiology: A Pattern Approach. (ed 3) Philadelphia: Lippincott, 1996, pp 119-124

2. Levine MS: Miscellaneous abnormalities of the esophagus. In: Gore RM, Levine MS: Textbook of Gastrointestinal Radiology. (ed 2) Philadelphia: WB Saunders, 2000, pp 465-483

\section{Gamut-10-3}

\section{DIVERTICULUM OF THE ESOPHAGUS- LOWER THIRD}

1. Epiphrenic ("lower Zenker's")

2. [Mucosal tear, spontaneous (Mallory-Weiss S.) or postinstrumentation]

3. [Penetrating peptic ulcer]

4. Postoperative (eg, for achalasia)

[ ] This condition does not actually cause the gamuted imaging finding, but can produce imaging changes that simulate it.

\section{References}

1. Bruggeman LL, Seaman WB: Epiphrenic diverticula: An analysis of 80 cases. AJR 1973; 119:266-276

2. Eisenberg RL: Gastrointestinal Radiology: A Pattern Approach. (ed 2) Philadelphia: Lippincott, 1996, pp 119-124

3. Kreel L: Outline of Radiology. New York: AppletonCentury-Crofts, 1971, pp 110-112

4. Reeders JW, Joosten FB, Rosenbusch G: Radiology of the esophagus. Radiologe 2000;40:479-493

\section{Gamut G-10-4}

\section{DIVERTICULA OF THE ESOPHAGUS- DIFFUSE}

1. Intramural diverticulosis

2. [Pseudodiverticulosis, esp. with Candida or herpes esophagitis]

[ ] This condition does not actually cause the gamuted imaging finding, but can produce imaging changes that simulate it. 


\section{References}

1. Eisenberg RL: Gastrointestinal Radiology: A Pattern Approach. (ed 2) Philadelphia: Lippincott, 1996, pp 119-124

2. Graham DY, Goyal RK, Sparkman J, et al: Diffuse intramural esophageal diverticulosis. Gastroenterology 1975; 68:781-785.

3. Levine MS, Moolten DN, Herlinger H, Laufer I: Esophageal intramural pseudodiverticulosis: a reevaluation. AJR 1986;147:1165-1170

4. Levine MS: Other esophagitides. In: Gore RM, Levine MS: Textbook of Gastrointestinal Radiology. (ed 2) Philadelphia: WB Saunders, 2000, pp 364-386

\section{Gamut G-11}

\section{SOLITARY INTRAMURAL OR INTRALUMINAL FILLING DEFECT OF THE ESOPHAGUS}

\section{COMMON}

1. Air bubble; meat impaction; coin; other foreign body

2. Carcinoma of esophagus or stomach

3. Extrinsic lesion invading the esophageal wall (eg, carcinoma of lung; granulomatous lymph node)

4. Gastrointestinal stromal tumor ${ }_{g}$ (esp. leiomyoma)

5. Papilloma, squamous

6. Plaque-like lesion (eg, candidiasis-usually multiple) (See G-17)

7. Polyp $_{\mathrm{g}}$ (eg, adenomatous; fibrovascular; inflammatory esophagogastric)

8. Varix

\section{UNCOMMON}

1. Abscess

2. Angioma

3. Duplication cyst (bronchogenic or enteric)

4. Hematoma, intramural

5. Lipoma

6. Lymphoma ${ }_{\mathrm{g}}$; leukemia

7. Melanoma

8. Metastasis
9. Prolapsed gastric fold

10. Sarcoma (eg, leiomyosarcoma; Kaposi sarcoma)

11. Ulcer with edema (See G-14)

12. Villous adenoma

\section{References}

1. Eisenberg RL: Gastrointestinal Radiology: A Pattern Approach. (ed 3) Philadelphia: Lippincott, 1996, pp 98-118

2. Gelfand DW: Gastrointestinal Radiology. New York: Churchill Livingstone, 1984, p 182

3. Levine MS: Esophagus: Differential diagnosis. In: Gore RM, Levine MS: Textbook of Gastrointestinal Radiology. (ed 2) Philadelphia: WB Saunders, 2000, pp 509-511

4. Styles RA, Gibb SP, Tarshis A, et al: Esophagogastric polyps: Radiographic and endoscopic findings. Radiology 1985;154:307-311

\section{Gamut G-12}

\section{LOCALIZED CONSTRICTION OR NARROWING OF THE ESOPHAGUS}

\section{COMMON}

1. Achalasia (cardiospasm)

2. Congenital atresia or stenosis, with or without T-E fistula

3. Duplication cyst (eg, bronchogenic cyst; gastroenteric cyst; neurenteric cyst)

4. Extrinsic pressure (eg, aortic knob; left main stem bronchus; aortic aneurysm or tortuosity, right aortic arch) (See G-5 to G-7)

5. Lower esophageal ring (Schatzki ring)

6. Neoplasm, benign (esp. gastrointestinal stromal tumor $_{\mathrm{g} \text {-leiomyoma; lipoma) }}$

7. Neoplasm, malignant (esp. carcinoma or leiomyosarcoma of esophagus or gastric cardia)

8. Physiologic (muscular ring; inferior esophageal sphincter; normal sling fibers of diaphragm)

9. Postoperative (fundoplication; repair of hiatal hernia or esophageal atresia); postgastrectomy alkaline reflux esophagitis

10. Spasm, localized (eg, lower esophageal spasm) 
11. Stricture (eg, peptic or reflux esophagitis; corrosive esophagitis-lye; oral medication; postradiation; nasogastric intubation; Barrett esophagus; congenital)

12. Web or diaphragm of esophagus

\section{UNCOMMON}

1. Benign mucous membrane pemphigoid

2. Cartilaginous ring (tracheobronchial rest)

3. Chronic granulomatous disease of childhood

4. Crohn's disease

5. Epidermolysis bullosa

6. Graft-versus-host disease

7. Hemorrhage in distal esophagus with adherent thrombus (eg, Mallory-Weiss S.; varices)

8. Hiatal hernia (esp. with short esophagus)

9. Infectious or inflammatory esophagitis (eg, candidiasis; herpes simplex; tuberculosis; histoplasmosis; actinomycosis; syphilis; eosinophilic esophagitis; Behçet S.)

10. Intramural esophageal pseudodiverticulosis

11. Lymphoma ${ }_{\mathrm{g}}$

12. Metastasis or direct spread from adjacent malignancy

13. Peptic esophageal ulcer

14. Postinstrumentation stricture (eg, nasogastric intubation; endoscopic perforation)

15. Sclerotherapy of esophageal varices

16. Tylosis (keratosis palmaris et plantaris familiaris)

17. Zollinger-Ellison S.

\section{References}

1. Eisenberg RL: Gastrointestinal Radiology: A Pattern Approach. (ed 3) Philadelphia: Lippincott, 1996, pp 70-97

2. Gelfand DW: Gastrointestinal Radiology. New York: Churchill Livingstone, 1984, p 188

3. Levine MS: Esophagus: Differential diagnosis. In: Gore RM, Levine MS: Textbook of Gastrointestinal Radiology. (ed 2) Philadelphia: WB Saunders, 2000, pp 509-511

4. Picus D, Frank PH: Eosinophilic esophagitis. AJR 1981; 136:1001-1003

5. Rohl L, Aksglaede K, Fundi-Jensen P, et al: Esophageal rings and strictures. Acta Radiol 2000;41:275-279

\section{Gamut G-13}

\section{THICKENING OF THE ESOPHAGEAL WALL (CT, US, MRI)}

\section{COMMON}

1. Carcinoma (squamous cell carcinoma in proximal 4/5; adenocarcinoma in distal fifth)

2. Corrosive esophagitis (eg, lye; oral medication)

3. Duplication cyst (eg, bronchogenic cyst; gastroenteric cyst; neurenteric cyst)

4. Infectious or inflammatory esophagitis (eg, candidiasis; herpes simplex; tuberculosis; histoplasmosis; actinomycosis; syphilis; eosinophilic esophagitis; Behçet S.)

5. Metastasis or direct spread from adjacent malignancy

6. Neoplasm, other (esp. gastrointestinal stromal

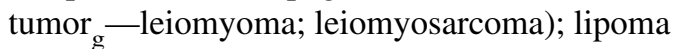

7. Postoperative (fundoplication; repair of hiatal hernia or esophageal atresia)

8. Reflux esophagitis (incl. Zollinger-Ellison S.; postgastrectomy alkaline reflux esophagitis)

9. Varices

\section{UNCOMMON}

1. Barrett esophagus

2. Benign mucous membrane pemphigoid

3. Chronic granulomatous disease of childhood

4. Crohn's disease

5. Epidermolysis bullosa

6. Hemorrhage or hematoma involving esophagus

7. [Hiatal hernia (esp. with short esophagus)]

8. Intramural esophageal pseudodiverticulosis

9. Lymphoma

10. Papillomatosis of esophagus

11. Peptic esophageal ulcer with edema

12. Tylosis (keratosis palmaris et plantaris familiaris)

[ ] This condition does not actually cause the gamuted imaging finding, but can produce imaging changes that simulate it.

\section{References}

1. Halpert RD, Feczko PJ: Gastrointestinal Radiology: The Requisites. (ed 2) St. Louis: Mosby-Year Book, 1999 
2. Levine MS: Esophagus: Differential diagnosis. In: Gore RM, Levine MS: Textbook of Gastrointestinal Radiology. (ed 2) Philadelphia: WB Saunders, 2000, pp 509-513

\section{Gamut G-14 ESOPHAGEAL ULCERATION(S)}

\section{COMMON}

1. Barrett esophagus

2. Carcinoma of esophagus

3. Corrosive esophagitis

4. Drug-induced esophagitis (eg, potassium chloride; tetracycline; quinidine; ascorbic acid; ferrous sulfate; bromide)

5. Intubation esophagitis

6. Opportunistic esophagitis (esp. Candida; herpes; cytomegalovirus)

7. Reflux or peptic esophagitis (eg, hiatal hernia; vomiting; chalasia; scleroderma; pregnancy; surgery)

\section{UNCOMMON}

1. Alcoholic esophagitis, acute

2. Behçet syndrome

3. Benign mucous pemphigoid

4. Eosinophilic esophagitis

5. Epidermolysis bullosa

6. Granulomatous esophagitis (eg, tuberculosis; histoplasmosis; syphilis; Crohn's disease)

7. Human immunodeficiency virus (HIV) infection

8. [Intramural esophageal pseudodiverticulosis]

9. Lymphoma

10. Metastasis

11. Radiation esophagitis

12. Sclerotherapy of esophageal varices

[ ] This condition does not actually cause the gamuted imaging finding, but can produce imaging changes that simulate it.

\section{References}

1. Berkovich GY, Levine MS, Miller WT: CT findings in patients with esophagitis. AJR 2000;175:1431-1434

2. Collazzo LA, Levine MS, Rubesin SE, et al: Acute radiation esophagitis: radiographic findings. AJR 1997;169:10671070
3. Eisenberg RL: Gastrointestinal Radiology: A Pattern Approach. (ed 3) Philadelphia: Lippincott, 1996, pp 45-69

4. Frager D, Kotler DP, Baer J. Idiopathic esophageal ulceration in the acquired immunodeficiency syndrome. Abdom Imaging 1994;19:2

5. Levine MS, Caroline D, Thompson JJ, et al: Adenocarcinoma of the esophagus: Relationship to Barrett mucosa. Radiology 1984;150:305-309

6. Levine MS: Esophagus: Differential diagnosis. In: Gore RM, Levine MS: Textbook of Gastrointestinal Radiology. (ed 2) Philadelphia: WB Saunders, 2000, pp 509-511

7. Sam JW, Levine MS, Rubesin SE, et al: The "foamy" esophagus: a radiographic sign of Candida esophagitis. AJR 2000; 174:999-1002

\section{Gamut G-15}

\section{TRANSVERSE MUCOSAL FOLDS IN THE ESOPHAGUS}

\section{COMMON}

1. Esophagitis (eg, reflux; candidiasis)

2. Normal (feline esophagus)

3. Scleroderma

4. [Tertiary contractions]

\section{UNCOMMON}

1. Achalasia

2. Corrosive esophagitis

3. [Linear transverse ulcerations]

[ ] This condition does not actually cause the gamuted imaging finding, but can produce imaging changes that simulate it.

\section{References}

1. Levine MS, Goldstein HM: Fixed transverse folds in the esophagus: a sign of reflux esophagitis. AJR 1984;143: 275-278

2. Levine MS: Infectious esophagitis. Semin Roentgenol 1994;24:341-350

3. Reeder JD, Kramer SS, Dudgeon DL: Transverse esophageal folds: Association with corrosive injury. Radiology 1985; 159:303-304

4. Reeders JW, Joosten FB, Rosenbusch G: Radiology of the esophagus. Radiologe 2000;40:479-493 


\section{Gamut G-16}

\section{WIDESPREAD IRREGULAR OR NODULAR ESOPHAGEAL MUCOSA}

\section{COMMON}

1. [Artifacts (esp. air bubbles)]

2. Corrosive esophagitis

3. Glycogenic acanthosis

4. Intubation esophagitis

5. Opportunistic esophagitis (eg, Candida; herpes; cytomegalovirus)

6. Peptic esophagitis (incl. Barrett type)

7. Reflux esophagitis (eg, hiatal hernia; chalasia; scleroderma)

8. Varices

\section{UNCOMMON}

1. Acanthosis nigricans

2. Behçet $S$.

3. Carcinoma, superficial spreading type

4. Cowden S. (multiple hamartoma S.)

5. Crohn's disease

6. Diverticulosis, intramural; pseudodiverticulosis

7. Ectopic sebaceous glands

8. Eosinophilic esophagitis

9. Epidermolysis bullosa

10. Esophagitis cystica

11. [Feline esophagus]

12. Granulomatous esophagitis (eg, tuberculosis; histoplasmosis; syphilis)

13. Hirsute esophagus ("skin tube" esophagus created during reconstructive surgery of pharynx and esophagus)

14. Leukoplakia

15. Lymph follicles

16. Lymphoma

17. Papillomatosis of esophagus

18. Pemphigus; bullous pemphigoid

19. Radiation esophagitis

20. Scleroderma

[ ] This condition does not actually cause the gamuted imaging finding, but can produce imaging changes that simulate it.
References

1. Gelfand DW: Gastrointestinal Radiology. New York: Churchill Livingstone, 1984, p 173

2. Itai Y, Kogure T, Okuyama Y: Radiological manifestations of oesophageal involvement in acanthosis nigricans. $\mathrm{Br} \mathbf{J}$ Radiol 1976:49:592-593

3. Itai Y, Kogure T, Okuyama Y, et al: Diffuse finely nodular lesions of the esophagus. AJR 1977;128:563-566

4. Levine MS: Esophagus: Differential diagnosis. In: Gore RM, Levine MS: Textbook of Gastrointestinal Radiology. (ed 2) Philadelphia: WB Saunders, 2000, pp 509-511

5. Meyers C, Durkin MG, Love L: Radiographic findings in herpetic esophagitis. Radiology 1976;119:21-22

\section{Gamut G-17}

\section{ESOPHAGEAL PLAQUES}

\section{COMMON}

1. Candidiasis (moniliasis)

\section{UNCOMMON}

1. Acanthosis nigricans

2. Barrett esophagus

3. Carcinoma, early or superficial spreading

4. Corrosive esophagitis

5. Crohn's disease

6. Leukoplakia

7. Reflux esophagitis

8. Tuberculosis

9. Viral esophagitis (herpes; cytomegalovirus)

\section{References}

1. Graziani L, Bearzi I, Romagnoli A, et al: Significance of diffuse granularity and nodularity of the esophageal mucosa at double-contrast radiography. Gastrointest Radiol 1985;10: $1-8$

2. Reeders JW, Joosten FB, Rosenbusch G: Radiology of the esophagus. Radiologe 2000;40:479-493 


\section{Gamut G-18}

\section{ESOPHAGEAL VARICES}

\section{COMMON}

1. Portal hypertension (esp. cirrhosis; portal vein thrombosis; schistosomiasis) (See G-191)

*2. Superior vena cava obstruction (downhill varices) (esp. bronchogenic carcinoma, mediastinal tumor or fibrosis; retrosternal goiter) (See E-70)

\section{UNCOMMON}

1. Arteriovenous malformation

2. Idiopathic

3. Noncirrhotic liver disease (eg, primary or metastatic carcinoma of liver; heart failure)

*4. Postsurgical (resection of retrosternal tumor)

5. [Varicoid lesions of esophagus (esp. varicoid carcinoma; lymphoma; esophagitis)] (See G-16)

* Varices may be confined to upper esophagus.

[ ] This condition does not actually cause the gamuted imaging finding, but can produce imaging changes that simulate it.

\section{References}

1. Baron RL, Gore RM: Diffuse liver disease. In: Gore RM, Levine MS: Textbook of Gastrointestinal Radiology. (ed 2) Philadelphia: WB Saunders, 2000, pp 1590-1638

2. Felson B, Lessure AP: "Downhill" varices of the esophagus. Dis Chest 1964;46:740

3. Eisenberg RL: Gastrointestinal Radiology: A Pattern Approach (ed 3). Philadelphia: Lippincott, 1996, pp 125-130

4. Reeders JW, Joosten FB, Rosenbusch G: Radiology of the esophagus. Radiologe 2000;40:479-493

\section{Gamut G-19}

\section{ESOPHAGEAL LESION IN A CHILD}

\section{COMMON}
1. Atresia
2. Chalasia (infantile gastroesophageal regurgitation)
3. Duplication cyst (enteric; neurenteric; bronchogenic)

4. Esophagitis (corrosive; reflux; peptic; radiation; infectious; intubation; instrumentation)

5. Extrinsic compression (See G-5-7)

6. Foreign body (esp. coin)

7. Hiatal hernia (esp. with short esophagus)

8. Opportunistic esophagitis (eg, Candida; herpes; cytomegalovirus)

9. Postsurgical repair of esophageal atresia, tracheoesophageal fistula, or hiatal hernia

10. Stricture (eg, congenital or secondary to esophagitis)

11. Vascular impression (eg, right aortic arch; double aortic arch; cervical aortic arch; aberrant right or left subclavian artery; pulmonary sling) (See G-7)

\section{UNCOMMON}

1. Achalasia

2. Congenital syndromes (eg, Riley-Day S. \{familial dysautonomia\}; Ehlers-Danlos S.)

3. Diverticulum (See G-10)

4. Epidermolysis bullosa

5. Metastasis

6. Neoplasm, benign or malignant (incl. lymphoma ${ }_{\mathrm{g}}$ )

7. Pemphigus

8. Peptic ulcer; Barrett esophagus

9. Trauma

10. Varices

11. Web; diaphragm; Schatzki ring

\section{References}

1. Fernbach SK, Zawin JK: Diseases of the pediatric esophagus. In: Gore RM, Levine MS: Textbook of Gastrointestinal Radiology. (ed 2) Philadelphia: WB Saunders, 2000, pp 2074-2089

2. Silverman FN, (ed:) Caffey's Pediatric X-ray Diagnosis: An Integrated Imaging Approach. (ed 8) Chicago: Year Book Medical Publ, 1985

3. Swischuk LE, John SD: Differential Diagnosis in Pediatric Radiology. (ed 2) Baltimore: Williams \& Wilkins, 1995, pp 175-176 


\section{Gamut G-20 \\ ABNORMAL POSITION OF THE STOMACH (ROTATION OR DISPLACEMENT)}

\section{COMMON}

1. Cascade stomach

2. Displacement by enlarged adjacent organ (eg, liver; spleen; left kidney; pancreas; aorta) or by adjacent mass, or lesser sac abscess or hernia

3. Eventration or paralysis of left hemidiaphragm

4. Hernia (eg, hiatal; paraesophageal; Morgagni; Bochdalek; traumatic; intrapericardial)

5. Inversion of left hemidiaphragm (pleural effusion; thoracic mass)

6. Obesity; emphysema (anterior displacement)

\section{UNCOMMON}

1. Absent hemidiaphragm

2. "Upside-down" stomach

3. Volvulus (organoaxial or mesenteroaxial)

\section{References}

1. Burgener FA, Kormano M: Differential Diagnosis in Conventional Radiology. (ed 2) New York: Thieme Medical Publ, 1991, pp 568-571

2. Kreel L: Outline of Radiology. New York: AppletonCentury-Crofts, 1971, pp 133-134

\section{Gamut G-21}

\section{FILLING DEFECT(S) IN THE STOMACH (INTRALUMINAL, MUCOSAL, OR INTRAMURAL)}

\section{COMMON}

*1. Adenomatous polyp (eg, in chronic atrophic gastritis; familial polyposis of colon; Cronkhite-Canada S.)

*2. [Areae gastricae]
3. Bezoar

*4. Blood clot; intramural hematoma

5. Carcinoma

6. Ectopic pancreas

7. [Extrinsic mass (eg, from spleen, liver, pancreas, kidney, colon)]

*8. Foreign body (eg, coin)

9. Giant rugal fold; hypertrophied prepyloric antral fold

*10. Hyperplastic polyp

*11. Leiomyoma, other gastrointestinal stromal tumor $_{\mathrm{g}}$

*12. Lymphoma

*13. Metastasis (esp. melanoma; Kaposi sarcoma; carcinoma of lung or breast)

*14. Neoplasm, other (eg, carcinoid; tubular adenoma; angioma; lipoma; villous adenoma; plasmacytoma)

*15. Peptic ulcer

16. Postoperative defect (eg, suture granuloma; fundoplication)

17. Sarcoma (esp. leiomyosarcoma)

*18. Varix

\section{UNCOMMON}

*1. Amyloidosis

*2. Candidiasis

3. [Double pylorus]

4. Duplication cyst

*5. Fundic gland polyposis

6. Gallstone

7. Granuloma with eosinophils (inflammatory fibroid polyp)

*8. Hamartoma (eg, Peutz-Jeghers S. (alimentary tract polyposis); Cowden S. (multiple hamartoma S.); Ruvalcaba-Myhre-Smith S.)

9. Jejunogastric intussusception

*10. Lymphoid hyperplasia; pseudolymphoma

*11. Parasites (esp. Ascaris; Anisakis)

12. Prolapse of esophageal mucosa

*13. Thickened folds simulating nodules or filling defects (eg, Ménétrier's disease; Crohn's disease; 
tuberculosis; sarcoidosis; eosinophilic gastritis)

(See G-26)

14. Tumefactive extramedullary hematopoiesis

* May be multiple.

[ ] This condition does not actually cause the gamuted imaging finding, but can produce imaging changes that simulate it.

\section{References}

1. Cherukuri R, Levine MS, Furth EE, et al: Giant hyperplastic polyps in the stomach: radiographic findings in seven patients. AJR 2000;175:1445-1448

2. Eisenberg RL: Gastrointestinal Radiology: A Pattern Approach. (ed 3) Philadelphia: Lippincott, 1996, pp 243-278

3. Gelfand DW: Gastrointestinal Radiology. New York: Churchill Livingstone, 1984, pp 221, 229

4. Lau CF, Hui PK, Mak KL, et al: Gastric polypoid lesions. Am J Gastroenterol 1998:93:2559-2564

5. Levine MS: Benign tumors of the stomach and duodenum. In: Gore RM, Levine MS: Textbook of Gastrointestinal Radiology. (ed 2) Philadelphia: WB Saunders, 2000, pp 575-600

6. Park SH, Han JK, Kim TK, et al: Unusual gastric tumors: radiologic-pathologic correlation. RadioGraphics 1999;19: 1435-1436

\section{Gamut G-22}

\section{FILLING DEFECT IN A GASTRIC REMNANT}

\section{COMMON}

1. Bezoar

2. Postoperative deformity or defect (eg, suture granuloma; scar; prolapse; mural inversion)

3. Ulcer (esp. marginal ulcer)

\section{UNCOMMON}

1. Carcinoma (recurrent; gastric stump)

2. Gastritis, postoperative (incl. bile reflux gastritis)

3. Gastrojejunal mucosal prolapse (antegrade prolapse into efferent or afferent loop)

4. Jejunogastric intussusception (usually efferent loop in Billroth II anastamosis)
5. Lymphoma (gastric stump)

6. Polyp (esp. hyperplastic)

\section{References}

1. Eisenberg RL: Gastrointestinal Radiology: A Pattern Approach. (ed 3) Philadelphia: Lippincott, 1996, pp 279-288

2. Smith CH, Gore RM: Postoperative stomach and duodenum. In: Gore RM, Levine MS: Textbook of Gastrointestinal Radiology. (ed 2) Philadelphia: WB Saunders, 2000, pp 682-697

\section{Gamut G-23}

\section{LESION INVOLVING THE GASTRIC FUNDUS (INTRINSIC OR EXTRINSIC)}

\section{COMMON}

1. Bezoar

2. Carcinoma of stomach (adenocarcinoma) or esophagus (squamous cell)

3. Diverticulum of fundus

4. Extragastric malignancy (eg, carcinoma of tail or body of pancreas or splenic flexure of colon; liver, kidney, or adrenal neoplasm; lymphoma ${ }_{\mathrm{g}}$ or metastases involving adjacent nodes)

5. Extrinsic pressure from normal or enlarged structure (eg, liver; spleen; splenic flexure of colon; left kidney; heart; aortic aneurysm)

6. Giant rugal folds (incl. Ménétrier's disease)

7. Hiatal hernia with esophagogastric herniation

8. Leiomyoma; neurofibroma; other gastrointestinal stromal tumor $_{\mathrm{g}}$; lipoma

9. Lymphoma

10. Peptic ulcer

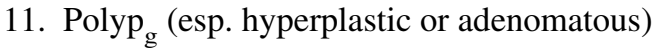

12. Postsurgical deformity (eg, Nissen repair of hiatal hernia with fundoplication; postsplenectomy)

13. Varices (eg, cirrhosis; schistosomiasis); portal hypertensive gastropathy 


\section{UNCOMMON}

1. Hematoma, intramural or extrinsic

2. Sarcoma (esp. leiomyosarcoma)

3. Splenosis following splenectomy

4. Subphrenic abscess

\section{References}

1. Chang R, Levine MS, Ginsberg GG, et al: Portal hypertensive gastropathy: radiographic findings in eight patients. AJR 2000;175:1609-1612

2. Eisenberg RL: Gastrointestinal Radiology: A Pattern Approach. (ed 3) Philadelphia: Lippincott, 1996, pp 305-316

3. Gore RM, Levine MS, Ghahremani GG, et al: Gastric cancer: radiologic diagnosis. Radiol Clin North Am 1997;35: $311-331$

\section{Gamut G-24}

\section{GASTRIC ULCERATION}

\section{COMMON}

1. Carcinoma of stomach

*2. Gastritis (eg, alcohol; aspirin; anti-inflammatory drugs)

3. Lymphoma

4. Marginal ulcer (postsubtotal gastrectomy)

5. Peptic ulcer

\section{UNCOMMON}

1. [Ectopic pancreas (duct)]

2. Carcinoid

3. Chemotherapy (hepatic arterial infusion)

4. Corrosive gastritis

*5. Crohn's disease

6. Cryptosporidium antritis

7. Eosinophilic gastritis

8. Kaposi sarcoma

9. Ménétrier's disease (giant hypertrophic gastritis)

10. Metastasis (often bull's-eye lesion, esp. melanoma) (See G-105)

11. Pseudolymphoma
12. Radiation therapy

13. Gastrointestinal stromal tumor ${ }_{g}$, benign or malignant (esp. leiomyoma; leiomyosarcoma; neurofibroma)

14. Suture line ulceration (esp. after gastric surgery for morbid obesity)

15. Tuberculosis

* May cause aphthoid ulcerations.

[ ] This condition does not actually cause the gamuted imaging finding, but can produce imaging changes that simulate it.

\section{References}

1. Eisenberg RL: Gastrointestinal Radiology: A Pattern Approach. (ed 3) Philadelphia: Lippincott, 1996, pp 181-203

2. Gelfand DW: Gastrointestinal Radiology. New York: Churchill Livingstone, 1984, p 216

3. Levine MS: Peptic ulcers. In: Gore RM, Levine MS: Textbook of Gastrointestinal Radiology. (ed 2) Philadelphia: WB Saunders, 2000, pp 509-513

\section{Gamut G-25 \\ EROSIVE GASTRITIS*}

\section{COMMON}

1. Acute gastritis (eg, alcohol abuse)

2. Crohn's disease

3. Drugs (eg, aspirin; NSAID; steroids)

4. Helicobacter pylori infection

5. Idiopathic

6. [Normal areae gastricae]

7. Peptic ulcer; hyperacidity

\section{UNCOMMON}

1. Corrosive gastritis

2. Cryptosporidium antritis

3. [Lymphoma]

4. Opportunistic infection (eg, candidiasis; herpes simplex; cytomegalovirus)

5. Postoperative gastritis 
6. Radiation therapy

7. Zollinger-Ellison S.; multiple endocrine neoplasia (MEN) S.

* Superficial erosions or aphthoid ulcerations seen especially with double contrast technique.

[ ] This condition does not actually cause the gamuted imaging finding, but can produce imaging changes that simulate it.

\section{References}

1. Eisenberg RL: Gastrointestinal Radiology: A Pattern Approach. (ed 3) Philadelphia: Lippincott, 1996, pp 204-207

2. Levine MS: Inflammatory conditions of the stomach and duodenum. In: Gore RM, Levine MS: Textbook of Gastrointestinal Radiology. (ed 2) Philadelphia: WB Saunders, 2000, pp 546-574

3. McLean AM, Paul RE Jr, Philips E: Chronic erosive gastritis-Clinical and radiologic features. J Can Assoc Radiol 1982;33:158-162

\section{Gamut G-26}

\section{LARGE GASTRIC FOLDS (LOCAL OR WIDESPREAD); ALSO THICKENING OF THE STOMACH WALL ON CT, US, OR MRI}

\section{COMMON}

1. Carcinoma

2. Gastritis (esp. hypertrophic; alcoholic; antral; Helicobacter pylori)

3. Lymphoma

4. Ménétrier's disease (giant hypertrophic gastritis)

5. Normal variant (hyperrugosity of fundus and greater curvature)

6. Pancreatitis, acute

7. Peptic ulcer disease; hyperacidity

8. Postoperative stomach

9. Varices (eg, cirrhosis; schistosomiasis); portal hypertensive gastropathy

\section{UNCOMMON}

1. Amyloidosis

2. Diffuse cystic gastric disease
3. Drug related gastritis (eg, aspirin; NSAID); chemotherapy toxicity

4. [Food retention]

5. Gastritis, other (eg, eosinophilic, corrosive, phlegmonous, or postradiation)

6. Granulomatous infiltration of stomach wall (eg, Crohn's disease; sarcoidosis; tuberculosis; histoplasmosis; actinomycosis; syphilis)

7. Infectious gastritis, other (eg, botulism; dysentery; diphtheria; candidiasis, cryptosporidiosis or cytomegalovirus-esp. in AIDS)

8. Metastasis; extension from carcinoma of pancreas

9. Neoplasm, other (eg, gastrointestinal stromal tumor $_{\mathrm{g}}$ - esp. leiomyoma, leiomyosarcoma; carcinoid; Kaposi sarcoma)

10. Parasitic disease (esp. strongyloidiasis; anisakiasis; schistosomiasis with varices)

11. Polyposis of stomach (See G-21, G-106)

12. Pseudolymphoma

13. Zollinger-Ellison S.; multiple endocrine neoplasia (MEN) S. (See J-5)

[ ] This condition does not actually cause the gamuted imaging finding, but can produce imaging changes that simulate it.

\section{References}

1. Chang MS, Levine MS, Ginsberg GG, et al: Portal hypertensive gastropathy: radiographic findings in eight patients. AJR 2000;175:1609-1612

2. Eisenberg RL: Gastrointestinal Radiology: A Pattern Approach. (ed 3 ) Philadelphia: Lippincott, 1996, pp 227-242

3. Farman J, Lerner ME, Ng C, et al: Cytomegalovirus gastritis. Gastrointest Radiol 1992;17:202

4. Levine MS: Stomach and duodenum: Differential diagnosis. In: Gore RM, Levine MS: Textbook of Gastrointestinal Radiology. (ed 2) Philadelphia: WB Saunders, 2000, pp 698-703

5. Levine MS, Laufer I: Stomach. In: Levine MS, Rubesin SE, Laufer I: Double Contrast Gastrointestinal Radiology. (ed 3) Philadelphia: WB Saunders, 2000, pp 149-203

6. Reese DF, Hodgson JR, Dockerty MB: Giant hypertrophy of the gastric mucosa (Ménétrier's disease): Correlation of the roentgenographic, pathologic and clinical findings. AJR 1962;88:619-626

7. Sato T, Sakai Y, Ishiguro S, Furukawa H: Radiologic manifestations of early gastric lymphoma. AJR 1986;146: $513-517$ 


\section{Gamut G-27}

\section{LINITIS PLASTICA PATTERN OF THE STOMACH (See G-28)}

\section{COMMON}

1. Carcinoma of stomach

2. Peptic ulcer (acute ulcer with spasm; chronic ulcer with fibrosis)

3. Stenosing antral gastritis

\section{UNCOMMON}

1. Amyloidosis

2. Chemotherapy (hepatic arterial infusion)

3. Corrosive gastritis (esp. acids; ferrous sulfate)

4. Eosinophilic gastritis

5. [Extrinsic mass compressing stomach (esp. marked hepatomegaly)]

6. Granulomatous infiltration of stomach wall (eg, Crohn's disease; sarcoidosis; tuberculosis; histoplasmosis; actinomycosis; syphilis)

7. Idiopathic gastritis

8. Intramural gastric hematoma

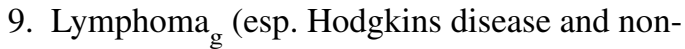
Hodgkins lymphoma)

10. Metastasis (esp. breast carcinoma); direct extension from carcinoma of pancreas or transverse colon; omental "cakes"

11. Opportunistic infection, esp. in AIDS (eg, cytomegalovirus; Cryptosporidium gastritis)

12. Parasitic disease (eg, strongyloidiasis; schistosomiasis)

13. Perigastric adhesions

14. Phlegmonous gastritis

15. Postoperative (eg, gastroplasty)

16. Postradiation or postfreezing gastritis

17. Pseudolymphoma

[ ] This condition does not actually cause the gamuted imaging finding, but can produce imaging changes that simulate it.

\section{References}

1. Balthazar EJ, Rosenberg H, Davidian MM: Scirrhous carcinoma of the pyloric channel and distal antrum. AJR 1980; 134:669-673
2. Eisenberg RL: Gastrointestinal Radiology: A Pattern Approach. (ed 3) Philadelphia: Lippincott, 1996, pp 208-226

3. Gelfand DW: Gastrointestinal Radiology. New York: Churchill Livingstone, 1984, p 229

4. Lagasse JP, Causse X, Legoux JL, et al: Cytomegalovirus gastritis simulating cancer of the linitis plastica type on endoscopic ultrasonography. Endoscopy 1998;30:S101-102

5. Levine MS: Stomach and duodenum: Differential diagnosis. In: Gore RM, Levine MS: Textbook of Gastrointestinal Radiology. (ed 2) Philadelphia: WB Saunders, 2000, pp 698-702

6. Levine MS, Laufer I: Tumors of the stomach. In: Levine MS, Rubesin SE, Laufer I: Double Contrast Gastrointestinal Radiology. (ed 3) Philadelphia: WB Saunders, 2000, pp 204-238

\section{Gamut G-28}

\section{NARROWING OR DEFORMITY OF THE GASTRIC ANTRUM (See G-27)}

\section{COMMON}

1. Antral gastritis

2. Carcinoma of stomach

3. Hypertrophic pyloric stenosis (infantile, adult)

4. Pancreatitis; carcinoma of pancreas; pseudocyst

5. Peptic ulcer scarring

6. Prolapse of gastric mucosa or polyp $\mathrm{p}_{\mathrm{g}}$

7. Pylorospasm

\section{UNCOMMON}

1. Aberrant pancreatic tissue

2. Adhesions

3. Amyloidosis

4. Antral diaphragm or web

5. Congenital peritoneal bands (Ladd's bands)

6. Corrosive gastritis

7. Cryptosporidium antritis

8. Duplication cyst of stomach

9. Eosinophilic gastritis

10. Gastroenterostomy

11. Granulomatous disease of infancy (neutrophil dysfunction) 
12. Granulomatous infiltration of stomach wall (eg, Crohn's disease; sarcoidosis; tuberculosis; histoplasmosis; actinomycosis; syphilis)

13. Lymphoma ${ }_{\mathrm{g}}$

14. Metastasis

15. Parasitic disease (esp. strongyloidiasis; schistosomiasis; anisakiasis)

16. Peptic ulcer perforation (walled off)

17. Radiation therapy

\section{References}

1. Gelfand DW: Gastrointestinal Radiology. New York: Churchill Livingstone, 1984, p 229

2. Levine MS: Stomach and duodenum: Differential diagnosis. In: Gore RM, Levine MS: Textbook of Gastrointestinal Radiology. (ed 2) Philadelphia: WB Saunders, 2000, pp 698-702

3. Teplick JG, Haskin ME: Roentgenologic Diagnosis. (ed 3) Philadelphia: WB Saunders, 1976

\section{Gamut G-29}

\section{COMBINED GASTRIC ANTRAL AND DUODENAL DISEASE}

\section{COMMON}

1. Carcinoma of stomach extending to involve duodenum

2. Involvement of stomach and duodenum from adjacent malignancy (esp. carcinoma of pancreas) or pancreatitis

3. Lymphoma

4. Peptic ulcer disease (incl. Zollinger-Ellison S.)

5. Prolapse of gastric mucosa, inflamed antral-pyloric fold, or polyp $\mathrm{g}_{\mathrm{g}}$ into duodenal bulb

\section{UNCOMMON}

1. Crohn's disease

2. Eosinophilic gastroenteritis

3. Ménétrier's disease
4. Parasitic disease (eg, strongyloidiasis; schistosomiasis)

5. Tuberculosis

\section{Reference}

1. Eisenberg RL: Gastrointestinal Radiology: A Pattern Approach. (ed 3) Philadelphia: Lippincott 1996, pp 324-328

2. Levine MS: Inflammatory conditions of the stomach and duodenum. In: Gore RM, Levine MS: Textbook of Gastrointestinal Radiology. (ed 2) Philadelphia: WB Saunders, 2000, pp 546-574

\section{Gamut G-30}

\section{GASTRIC OUTLET OBSTRUCTION}

\section{COMMON}

1. Extrinsic compression (eg, pancreatic, renal, retroperitoneal, duodenal, or colonic lesion)

2. Neoplasm, esp. malignant (eg, carcinoma of gastric antrum or head of pancreas; lymphoma $\mathrm{g}_{\mathrm{g}}$ )

3. Peptic ulcer disease (eg, antral, pyloric, or duodenal)

4. [Physiologic (eg, gastric atony with poor peristalsis and emptying; post-drug therapy; gastric distention)] (See G-31)

5. Prepyloric inflammation, scarring or stricture (eg, corrosive gastritis; Crohn's disease; tuberculosis; sarcoidosis; syphilis; amyloidosis)

6. Pyloric hypertrophy, adult or infantile (hypertrophic pyloric stenosis)

7. Pylorospasm

\section{UNCOMMON}

1. Annular pancreas

2. Bezoar

3. Diaphragm or web, antral or duodenal

4. Gastric duplication

5. Hematoma, intramural

6. Intussusception, gastroduodenal

7. Pancreatitis; cholecystitis 
8. Prolapsed antral mucosa or polyp

9. [Proximal small bowel obstruction]

10. Volvulus (with or without hiatal hernia)

[ ] This condition does not actually cause the gamuted imaging finding, but can produce imaging changes that simulate it.

\section{References}

1. Eisenberg RL: Gastrointestinal Radiology: A Pattern Approach. (ed 3) Philadelphia: Lippincott, 1996, pp 289-298

2. Teplick JG, Haskin ME: Roentgenologic Diagnosis. (ed 3) Philadelphia: WB Saunders, 1976

\section{Gamut G-31}

\section{DILATATION OF THE STOMACH WITHOUT OBSTRUCTION}

\section{COMMON}

1. Aerophagia; emotional distress; hyperventilation; crying

2. Carbonated beverages; bicarbonate of soda; double contrast; gas pills

3. Coma (uremic or hepatic)

4. Diabetic gastropathy (gastric paresis)

5. Drug therapy (eg, Atropine; morphine; ganglionblocking agent; Pro-Banthine)

6. Gastritis, acute

7. Iatrogenic (intubation; oxygen tube in esophagus)

8. Immobilization (eg, body cast; paraplegia)

9. Inflammation, acute (eg, pancreatitis; cholecystitis; subphrenic abscess; septicemia)

10. Pain (eg, colic due to renal, ureteral, or biliary stone; porphyria; lead poisoning; sickle cell crisis; migraine)

11. Peritonitis (eg, perforated appendix or peptic ulcer)

12. Postoperative, recent (incl. vagotomy)

13. Small bowel obstruction, proximal

14. Traumatic gastric ileus (eg, spine fracture; ruptured spleen; retroperitoneal hematoma; renal injury)

\section{UNCOMMON}

1. Chagas' disease

2. Electrolyte or acid-base imbalance (eg, hypercalcemia; hypocalcemia; hypokalemia; uremia; insulin shock; diabetic ketoacidosis)

3. Idiopathic

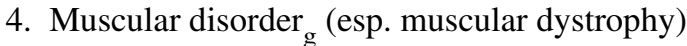

5. Myxedema (hypothyroidism)

6. Neurologic disorder ${ }_{\mathrm{g}}$ (eg, brain tumor; cerebral palsy; bulbar poliomyelitis; tabes dorsalis)

7. Scleroderma; dermatomyositis

8. Tracheoesophageal fistula with esophageal atresia

\section{References}

1. Eisenberg RL: Gastrointestinal Radiology: A Pattern Approach. (ed 3) Philadelphia: Lippincott, 1996, pp 300-304

2. Marie I, Levesque H, Ducrotté P, et al: Gastric involvement in systemic sclerosis. Am J Gastroenterol 2001:96: $77-81$

\section{Gamut G-32 \\ INTERSTITIAL EMPHYSEMA OF THE STOMACH}

\section{COMMON}

1. Emphysematous or phlegmonous gastritis (gas-forming organism, esp. in diabetic or alcoholic)

2. Traumatic or iatrogenic emphysema (eg, gastroscopy; intubation; recent surgery; respiratory therapy-esp. PEEP)

\section{UNCOMMON}

1. Corrosive gastritis

2. Distention of stomach (eg, bicarbonate of soda; oxygen tube in esophagus) (See G-31)

3. Gastric outlet obstruction (eg, malignancy; volvulus; prepyloric inflammation or stricture) (See G-30)

4. Ischemic gastritis; infarction 
5. Necrotizing gastroenterocolitis

6. Peptic ulcer with intramural perforation

7. Perforated appendicitis

8. Pneumatosis cystoides

9. Pneumomediastinum (eg, from emphysema or asthma)

\section{References}

1. Eisenberg RL: Gastrointestinal Radiology: A Pattern Approach. (ed 3) Philadelphia: Lippincott, 1996, pp 320-323

2. Tuck JS, Boobis LH: Case report: Interstitial emphysema of the stomach due to perforated appendicitis. Clin Radiol 1987;38:315-317

3. Vaughn BF: Emphysema of the stomach with portal vein gas. Australas Radiol 1972;16:377-378

\section{Gamut G-33}

\section{GASTROCOLIC OR}

\section{GASTRODUODENOCOLIC FISTULA}

\section{COMMON}

1. Carcinoma of colon, stomach, pancreas, or kidney

2. Pancreatitis; pancreatic abscess

3. Peptic ulcer, perforated (incl. aspirin or NSAIDinduced greater curvature ulcer)

4. Postoperative (eg, gastrostomy; retained sponge)

5. Trauma with gastric, duodenal, or colonic perforation

\section{UNCOMMON}

1. Actinomycosis

2. Amebic colitis

3. Biliary tract perforation (eg, calculus)

4. Crohn's disease

5. Diverticulum, perforated (eg, colonic, duodenal)

6. Foreign body

7. Idiopathic

8. Lymphoma $_{\mathrm{g}}$

9. Marginal ulcer

10. Metastatic disease
11. Tuberculosis

12. Typhoid fever

\section{References}

1. Alexander ES, Weinberg S, Clark RA, et al: Fistulas and sinus tracts: Radiographic evaluation, management, and outcome. Gastrointest Radiol 1982;7:135-140

2. Laufer I, Joffe N, Stolberg H: Unusual causes of gastrocolic fistula. Gastrointest Radiol 1977;2:21-25

3. Levine MS: Inflammatory conditions of the stomach and duodenum. In: Gore RM, Levine MS: Textbook of Gastrointestinal Radiology. (ed 2) Philadelphia: WB Saunders, 2000, pp 546-574

\section{Gamut G-34}

\section{INCREASED RETROGASTRIC OR RETRODUODENAL SPACE}

\section{COMMON}

1. Ascites

2. Hepatomegaly, marked (esp. caudate lobe)

3. Normal variant

4. Obesity

5. Pancreatic mass (esp. carcinoma; pancreatitis; abscess; pseudocyst; cystadenoma) (See G-213, 214)

6. Renal or adrenal mass (eg, renal cell carcinoma (hypernephroma); renal cyst; hydronephrosis; adenoma or carcinoma of adrenal gland; pheochromocytoma; neuroblastoma; Wilms' tumor; perinephric abscess or hematoma) (See H-38-44, H-118)

7. Retroperitoneal mass (eg, sarcoma; lymphoma ${ }_{\mathrm{g}}$; metastasis; lymphadenopathy; tuberculosis; cyst; abscess; hematoma)

\section{UNCOMMON}

1. Aortic aneurysm

2. Choledochal cyst

3. Gastrointestinal stromal tumor ${ }_{\mathrm{g}}$ of posterior wall of stomach (esp. leiomyoma; leiomyosarcoma)

4. Herniation of omentum (eg, Morgagni hernia) 
5. Postoperative

6. Retroperitoneal edema, cellulitis, urinary leakage

7. Retroperitoneal fibrosis

\section{Reference}

1. Eisenberg RL: Gastrointestinal Radiology: A Pattern Approach (ed 3). Philadelphia: Lippincott, 1996, pp 317-319

\section{Gamut G-35}

\section{EXTRINSIC INDENTATION ON THE DUODENUM (See G-36)}

\section{COMMON}

1. Coloduodenal apposition

2. Colon lesion (esp. carcinoma of hepatic flexure or transverse colon; amebic pericolic abscess or ameboma)

3. Common duct dilatation or neoplasm (See G-130, G-134)

4. Duodenal diverticulitis or abscess (esp. with giant duodenal diverticulum)

5. Gallbladder, normal or enlarged (eg, hydrops; carcinoma; "Courvoisier gallbladder")

6. Hematoma, intramural or mesenteric

7. Hepatic enlargement (esp. caudate lobe) (eg, liver abscess; hepatocellular carcinoma \{hepatoma\} (See G-141)

8. Renal or adrenal mass (eg, renal cell carcinoma \{hypernephroma \}; renal cyst; hydronephrosis; adenoma or carcinoma of adrenal gland; pheochromocytoma; neuroblastoma; Wilms' tumor; perinephric abscess or hematoma) (See H-38-44, 118)

9. Lymph node enlargement (eg, metastasis; lymphoma $_{\mathrm{g}}$; tuberculosis)

10. Pancreatic mass (eg, carcinoma; pseudocyst; pancreatitis; abscess; annular pancreas) (See G-213, 214)

11. Papilla of Vater enlargement (See G-138)

12. [Postbulbar peptic ulcer]
13. Retroperitoneal mass (eg, sarcoma; lymphadenopathy; abscess; cyst)

14. Superior mesenteric artery compression (See G-44)

\section{UNCOMMON}

1. Aortic aneurysm

2. Choledochal cyst

3. Congenital peritoneal bands (Ladd's bands)

4. Gastric neoplasm (esp. leiomyosarcoma; carcinoma)

5. Idiopathic

6. Mesenteric or celiac artery collaterals

7. Pericholecystic abscess

8. Varices, duodenal or retroperitoneal

[ ] This condition does not actually cause the gamuted imaging finding, but can produce imaging changes that simulate it.

\section{Reference}

1. Eisenberg RL: Gastrointestinal Radiology: A Pattern Approach. (ed 3) Philadelphia: Lippincott, 1996, pp 365-369

\section{Gamut G-36 \\ WIDENING OF THE DUODENAL C-LOOP (See G-35)}

\section{COMMON}

1. Normal variant

2. Pancreatic mass (eg, acute or chronic pancreatitis; abscess; pseudocyst; carcinoma; cystadenoma; cystadenocarcinoma) (See G-213, 214)

\section{UNCOMMON}

1. Aortic aneurysm

2. Choledochal cyst

3. Duodenal diverticulitis

4. Duodenal hematoma

5. Gastrointestinal stromal tumor ${ }_{\mathrm{g}}$ of duodenum (esp. leiomyosarcoma)

6. Mesenteric or celiac artery collaterals 
7. Mesenteric or omental mass (eg, metastasis; hematoma; cystic lymphangioma)

8. Neoplasm of stomach, colon, or kidney (esp. with spread to head of pancreas)

9. Parasitic disease (eg, strongyloidiasis; amebiasis with pericolic abscess)

10. Retroperitoneal lymphadenopathy (eg, metastasis; lymphoma $_{\mathrm{g}}$; tuberculosis; sarcoidosis)

11. Retroperitoneal cyst or neoplasm, primary or metastatic

\section{Reference}

1. Eisenberg RL: Gastrointestinal Radiology: A Pattern Approach. (ed 3) Philadelphia: Lippincott, 1996, pp 348-364

\section{Gamut G-37
SOLITARY INTRINSIC
DUODENAL MASS}

\section{COMMON}

1. Ectopic pancreas

2. Metastasis (esp. hypernephroma; melanoma)

3. Neoplasm, primary (Brunner's gland adenoma; leiomyoma; neurofibroma; lipoma; myxoma; hamartoma; hemangioma; lymphangioma; islet cell tumor; carcinoid; villous adenoma)

4. Normal variant (eg, redundant duodenal fold; flexure "defect"; normal papilla of Vater)

5. Pancreatic lesion, attached or invading (esp. carcinoma of pancreas; pancreatitis; pseudocyst) (See G-213, 214)

6. Papilla of Vater enlargement (See G-138)

7. Peptic ulcer with edema or deformity

8. Polyp

9. Prolapsed gastric mucosa or polyp
4. Cyst (eg, duplication; choledochalcholedochocele)

5. Foreign body in lumen (eg, fruit pit)

6. Gallstone impaction at papilla

7. Intraluminal diverticulum

8. Intramural hematoma

9. Intussusception, gastroduodenal

10. Lymphoma $_{\mathrm{g}}$

11. Mesenteric or celiac artery collateral

12. Parasite (eg, Ascaris)

13. Postoperative defect; stitch abscess; prolapsed gastrostomy tube

14. Sarcoma (gastrointestinal stromal tumor ${ }_{g}$, esp. leiomyosarcoma; Kaposi sarcoma)

15. Varix

\section{References}

1. Eisenberg RL: Gastrointestinal Radiology: A Pattern Approach. (ed 3) Philadelphia: Lippincott, 1996, pp 370-396

2. Gelfand DW: Gastrointestinal Radiology. New York: Churchill Livingstone, 1984, p 254

3. Levine MS: Benign tumors of the stomach and duodenum. In: Gore RM, Levine MS: Textbook of Gastrointestinal Radiology. (ed 2) Philadelphia: WB Saunders, 2000, pp 575-600

\section{Gamut G-38 \\ MULTIPLE OR DIFFUSE FILLING DEFECTS IN THE DUODENUM}

\section{COMMON}

1. Brunner's gland hyperplasia

2. Heterotopic gastric mucosa

3. Metastases (esp. malignant melanoma)

4. Nodular, thickened, or edematous folds (incl. nonerosive duodenitis) (See G-40)

5. Prolapsed gastric mucosa

\section{UNCOMMON}

1. Abscess, juxtaduodenal

2. Blood clot

3. Carcinoma of duodenum or ampulla

(continued) 


\section{UNCOMMON}

1. Ascariasis

2. Blood clots

3. Crohn's disease (cobblestone pattern)

4. Cronkhite-Canada S.

5. Foreign bodies in lumen (eg, fruit pits)

6. Lymphoma ${ }_{\mathrm{g}}$; Kaposi sarcoma; other bull's-eye lesions (See G-105)

7. Mastocytosis

8. Mesenteric or celiac artery collaterals

9. Nodular lymphoid hyperplasia (esp. in dysgammaglobulinemia)

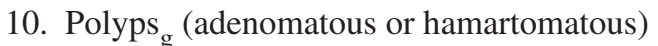

11. Varices

\section{Reference}

1. Glick SN, Gohel VK, Laufer I: Mucosal surface patterns of the duodenal bulb. Radiology 1984;150:317-322

2. Levine MS, Laufer I, Stevenson G: Duodenum. In: Levine MS, Rubesin SE, Laufer I: Double Contrast Gastrointestinal Radiology. (ed 3) Philadelphia: WB Saunders, 2000, pp 239-274

\section{Gamut G-39}

\section{DIMINISHED OR ABSENT FOLD PATTERN IN THE DUODENUM AND SMALL BOWEL}

\section{COMMON}

1. Crohn's disease

2. Scleroderma

3. Small bowel obstruction or ileus

4. Sprue

\section{UNCOMMON}

1. Amyloidosis

2. Cystic fibrosis (mucoviscidosis)

3. Strongyloidiasis, chronic

\section{Gamut G-40}

\section{NODULAR OR THICKENED FOLDS IN THE DUODENUM; ALSO THICKENING OF THE DUODENAL WALL ON CT, US, OR MRI}

\section{COMMON}

1. Brunner's gland hyperplasia

2. Cystic fibrosis (mucoviscidosis)

3. Duodenitis

4. Edema (eg, hypoproteinemia; cirrhosis; nephrotic S.; uremia; chronic dialysis; angioneurotic edema; heart failure)

5. Metastatic disease or direct invasion from adjacent neoplasm (esp. carcinoma of pancreas, stomach, or colon)

6. Normal variant

7. Pancreatitis, acute or chronic

8. Parasitic disease (esp. giardiasis; strongyloidiasis; hookworm disease; intestinal capillariasis)

9. Peptic ulcer disease

10. Zollinger-Ellison S.; multiple endocrine neoplasia (MEN) S. (See J-5)

\section{UNCOMMON}

1. AIDS-related infection (eg, Cryptosporidium; cytomegalovirus; atypical mycobacterial infection) or neoplasm (eg, Kaposi sarcoma; lymphoma ${ }_{\mathrm{g}}$ )

2. Amyloidosis

3. Cholecystitis

4. Corrosive disease

5. Crohn's disease

6. Cronkhite-Canada S.

7. Drug therapy (eg, Pro-Banthine); chemotherapy toxicity

8. Duodenal diverticulitis

9. Ectopic gastric mucosa

10. Eosinophilic enteritis

11. Intestinal lymphangiectasia

12. Intramural hemorrhage (eg, trauma; hemophilia or other bleeding disorder ${ }_{\mathrm{g}}$; anticoagulant therapy) 
13. Ischemia; vasculitis (eg, connective tissue disease (collagen vascular disease) ${ }_{\mathrm{g}}$; Henoch-Schönlein purpura)

14. Neoplasm, primary (eg, carcinoma; lymphoma ; Kaposi sarcoma; gastrointestinal stromal tumor ${ }_{\mathrm{g}}$ esp. leiomyoma, leiomyosarcoma, or neurofibroma; lipoma; carcinoid)

15. Mastocytosis

16. Ménétrier's disease

17. Mesenteric or celiac artery collaterals following occlusion of main trunks

18. Nodular lymphoid hyperplasia (esp. in dysgammaglobulinemia)

19. Postoperative

20. Radiation injury, acute or chronic

21. Sprue; celiac disease

22. Tuberculosis

23. Varices

24. Whipple's disease

\section{References}

1. Eisenberg RL: Gastrointestinal Radiology: A Pattern Approach. (ed 3) Philadelphia: Lippincott, 1996, pp 337-347

2. Glick SN, Gohel VK, Laufer I: Mucosal surface patterns of the duodenal bulb. Radiology 1984;150:317-322

3. Halpert RD, Feczko PJ: Gastrointestinal Radiology: The Requisites. (ed 2) St. Louis: Mosby-Year Book, 1999

4. Levine MS, Laufer I, Stevenson G: Duodenum. In: Levine MS, Rubesin SE, Laufer I: Double Contrast Gastrointestinal Radiology. (ed 3) Philadelphia: WB Saunders, 2000, pp 239-274

5. Levine MS: Stomach and duodenum: Differential diagnosis. In: Gore RM, Levine MS: Textbook of Gastrointestinal Radiology. (ed 2) Philadelphia: WB Saunders, 2000, pp 698-703

6. Rubesin SE, Herlinger H: Small bowel: Differential diagnosis. In: Gore RM, Levine MS: Textbook of Gastrointestinal Radiology. (ed 2) Philadelphia: WB Saunders, 2000, pp 884-891

\section{Gamut G-41 \\ POSTBULBAR DUODENAL ULCERATION}

\section{COMMON}

1. [Diverticulum]

2. Neoplasm, malignant, extrinsic (eg, invasion from pancreas, colon, right kidney, or gallbladder)

3. Peptic ulcer

4. Zollinger-Ellison S.

\section{UNCOMMON}

1. Aorticoduodenal fistula (esp. aortic graft)

2. Carcinoma of duodenum

3. Ectopic pancreas

4. Fistula, duodenocolic or other (See G-107)

5. Gastrointestinal stromal tumor ${ }_{\mathrm{g}}$ (esp. leiomyoma; leiomyosarcoma; neurofibroma)

6. Granulomatous disease (eg, Crohn's disease; tuberculosis)

7. Intramural duodenal pseudodiverticulosis

8. Lymphoma $_{\mathrm{g}}$

9. Metastasis (eg, melanoma; Kaposi sarcoma)

10. Parasitic disease (eg, strongyloidiasis)

[ ] This condition does not actually cause the gamuted imaging finding, but can produce imaging changes that simulate it

\section{References}

1. Eisenberg RL: Gastrointestinal Radiology: A Pattern Approach (ed 3). Philadelphia: Lippincott, 1996, pp 331-336

2. Gelfand DW: Gastrointestinal Radiology. New York: Churchill Livingstone, 1984, p 243

3. Solomon DJ, Kottler RE: Duodenal intramural pseudodiverticulosis. Gastrointest Radiol 1992;17:217 


\section{Gamut G-42}

\section{DUODENAL NARROWING OR OBSTRUCTION (See G-43, 44)}

\section{COMMON}

1. Congenital atresia, esp. with trisomy $21 \mathrm{~S}$. (Down S.), stenosis, diaphragm or web

2. Extrinsic mass (eg, mesenteric or para-aortic lymphadenopathy; invasive neoplasm from pancreas, kidney, or colon; aortic aneurysm; choledochal cyst)

3. Pancreatitis, acute or chronic; pseudocyst

4. Postbulbar duodenal ulcer or scar

5. Superior mesenteric artery syndrome (See G-44)

\section{UNCOMMON}

1. Adhesions

2. Annular pancreas

3. Aorticoduodenal fistula

4. Cholecystitis

5. Congenital peritoneal bands (Ladd's bands)

6. Duplication cyst

7. Gallstone impaction

8. Hematoma (intramural or extrinsic)

9. Inflammatory disease of duodenum (eg, Crohn's disease; tuberculosis; strongyloidiasis; giardiasis with spasm)

10. Internal hernia (eg, paraduodenal)

11. Intraluminal diverticulum

12. Midgut volvulus with malrotation

13. Neoplasm of duodenum, primary (esp. carcinoma; leiomyosarcoma) or metastatic; also Burkitt lymphoma

14. Preduodenal portal vein

15. Prolapsed gastric lesion (eg, polyp $\mathrm{p}_{\mathrm{g}}$ )

16. Pseudo-obstruction, idiopathic

17. Stricture (eg, traumatic; radiation)

\section{Gamut G-43}

\section{DUODENAL OBSTRUCTION IN AN INFANT (DOUBLE BUBBLE SIGN)}

\section{COMMON}

1. Annular pancreas

2. Congenital peritoneal bands (Ladd's bands)

3. Duodenal atresia or stenosis, esp. with trisomy $21 \mathrm{~S}$. (Down S.)

4. Midgut volvulus with malrotation

\section{UNCOMMON}

1. Choledochal cyst

2. Diaphragm or web; intraluminal diverticulum

3. Duplication cyst

4. Intramural hematoma

5. Preduodenal portal vein

6. Retroperitoneal tumor (eg, teratoma) or lymphadenopathy

\section{References}

1. Kassner EG, Sutton AL, DeGroot TJ: Bile duct anomalies associated with duodenal atresia; paradoxical presence of small bowel gas. AJR 1972;116:577-583

2. Silverman FN (ed): Caffey's Pediatric X-ray Diagnosis: An Integrated Imaging Approach. (ed 8) Chicago: Year Book Medical Publ, 1985

3. Swischuk LE: Imaging of the Newborn, Infant, and Young Child. (ed 3) Baltimore: Williams \& Wilkins, 1989

4. Teele RL, Share JC: Diseases of the pediatric stomach and duodenum. In: Gore RM, Levine MS: Textbook of Gastrointestinal Radiology. (ed 2) Philadelphia: WB Saunders, 2000, pp 2090-2107 


\section{Gamut G-44}

SUPERIOR MESENTERIC ARTERY SYNDROME (BAND-LIKE CONSTRICTION OF TRANSVERSE DUODENUM)

\section{COMMON}

1. Immobilization or prolonged bed rest (eg, postsurgery; severe burn; body cast)

2. Normal variant

3. Pancreatic mass; pancreatitis

4. Scleroderma; dermatomyositis; lupus erythematosus

5. Severe weight loss with loss of retroperitoneal fat

\section{UNCOMMON}

1. Adhesions; congenital peritoneal bands (Ladd's bands)

2. Aortic aneurysm; postrepair aorticoduodenal fistula

3. Chronic idiopathic intestinal pseudo-obstruction

4. Internal hernia, paraduodenal

5. Loss of abdominal muscle tone (eg, multiple pregnancies)

6. Retroperitoneal inflammatory or neoplastic disease

7. Small vascular (aorticomesenteric) angle

8. Thickening of root of mesentery (eg, Crohn's disease; pancreatitis; tuberculosis; metastatic disease; other lymphadenopathy)

\section{References}

1. Anderson JR, Earnshaw PM, Fraser GM: Extrinsic compression of the third part of the duodenum. Clin Radiol 1982;33:75-81

2. Eisenberg RL: Gastrointestinal Radiology: A Pattern Approach. (ed 3) Philadelphia: Lippincott, 1996, pp 411-417

3. Wallace RG, Howard WB: Acute superior mesenteric artery syndrome in the severely burned patient. Radiology 1970;94:307-310

\section{Gamut G-45}

\section{DUODENAL DILATATION WITHOUT OBSTRUCTION}

\section{COMMON}

1. Drug therapy (eg, Pro-Banthine; atropine; morphine; Lomotil)

2. Idiopathic

3. Ileus, localized (eg, acute pancreatitis; cholecystitis; peptic ulcer disease; severe trauma or burn)

4. Immobilization (eg, body cast; burn; paraplegia)

5. Normal variant

6. Postoperative (incl. vagotomy)

7. Scleroderma; dermatomyositis; lupus erythematosus

\section{UNCOMMON}

1. Chagas' disease (aganglionosis)

2. Diabetes (incl. acidosis; coma; insulin shock)

3. Emotional state alteration; hyperventilation; aerophagia

4. Pain (eg, lead colic; tabetic crisis; porphyria)

5. Sprue; other malabsorption syndromes

6. Thiamine deficiency neuropathy

7. Zollinger-Ellison S.

\section{Reference}

1. Levine MS: Stomach and duodenum: Differential diagnosis. In: Gore RM, Levine MS: Textbook of Gastrointestinal Radiology. (ed 2) Philadelphia: WB Saunders, 2000, pp 698-702

\section{Gamut G-46}

\section{ABNORMAL POSITION OF SMALL BOWEL LOOPS}

\section{COMMON}

1. Anterior abdominal hernia (eg, umbilical; ventral; postoperative incisional)

2. Inguinal or femoral hernia

(continued) 
3. Lesser sac hernia

4. Malrotation (incl. midgut volvulus)

5. Paraduodenal hernia (right or esp. left)

\section{UNCOMMON}

1. Diaphragmatic hernia

2. Internal hernias (pericecal; small bowel mesentery; sigmoid mesentery; pelvic [broad ligament])

3. Obturator hernia (esp. on right)

4. Omphalocele; Cantrell S.

5. Spigelian hernia

\section{References}

1. Burgener FA, Kormano M: Differential Diagnosis in Conventional Radiology. (ed 2) New York: Thieme Medical Publ, 1991, pp 576577

2. Eisenberg RL: Gastrointestinal Radiology: A Pattern Approach. (ed 3) Philadelphia: Lippincott,1996, pp 909-924

\section{Gamut G-47}

\section{SEPARATION OR DISPLACEMENT OF SMALL BOWEL LOOPS}

\section{COMMON}

1. Abscess, intraperitoneal (eg, appendiceal; diverticular; interloop)

2. Adhesions

3. Ascites or other peritoneal fluid (eg, cirrhosis; congestive heart failure; peritoneal carcinomatosis)

4. Bladder enlargement

5. Crohn's disease

6. Intestinal neoplasm, primary (eg, carcinoid or carcinoma of small bowel)

7. Lymphadenopathy in mesentery or retroperitoneum

8. Mesenteric mass (eg, cyst; leiomyosarcoma; lipomatosis; lymphangioma) (See G-228, 229)

9. Metastatic disease to mesentery, bowel, peritoneum, or retroperitoneal nodes

10. Neoplasm or cyst in abdomen, pelvis or retroperitoneum, other (eg, gastrointestinal stromal tumor $_{\mathrm{g}}$; ovarian cyst or tumor; retroperitoneal sarcoma; renal mass; pancreatic pseudocyst; hydatid cyst; mesothelioma; plexiform neurofibroma)

11. Peritonitis (eg, bacterial; tuberculous; typhoid fever)

12. Postoperative (eg, resection)

\section{UNCOMMON}

1. Amyloidosis

2. Graft-versus-host disease

3. Hematoma or hemorrhage (trauma or bleeding disorder ${ }_{\mathrm{g}}$ involving abdominal wall, mesentery, or bowel wall)

4. Hernia (internal; retroperitoneal)

5. Lymphoma

6. Mesenteric infarction

7. Mesenteritis (eg, retractile); Weber-Christian disease

8. Radiation enteritis

9. Tuberculosis

10. Whipple's disease

\section{References}

1. Burgener FA, Kormano M: Differential Diagnosis in Conventional Radiology. (ed 2) New York: Thieme Medical Publ, 1991, pp 574-577

2. Eisenberg RL: Gastrointestinal Radiology: A Pattern Approach. (ed 3) Philadelphia: Lippincott, 1996, pp 529-540

\section{Gamut G-48}

\section{SOLITARY MASS IN THE SMALL BOWEL WITH PRESERVED MUCOSA}

\section{COMMON}

1. Benign neoplasm (eg, adenoma; angioma; hamartoma; lipoma; leiomyoma, neurofibroma or other gastrointestinal stromal tumor ${ }_{\mathrm{g}}$ )

2. Carcinoid (esp. in ileum)

3. Food particle; fruit pit; bezoar; enterolith; pill; foreign body

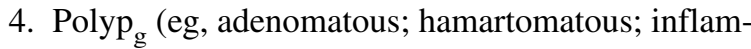
matory fibroid) 


\section{UNCOMMON}

1. Carcinoma, early or atypical (usually destroys mucosa)

2. Cyst (eg, duplication)

3. Endometrial implant

4. Gallstone

5. Heterotopic gastric mucosa; ectopic pancreas

6. Intraluminal diverticulum

7. Intramural hematoma; blood clot

8. Inverted Meckel's diverticulum

9. Lymphoma

10. Meconium ileus (cystic fibrosis \{mucoviscidosis

11. Metastasis (esp. melanoma)

12. Parasite (eg, Ascaris bolus; tapeworm-Taenia aginata or T. solium; Anisakis with ileocecal phlegmon)

13. Sarcoma (eg, leiomyosarcoma; Kaposi sarcoma)

14. Varix

\section{Reference}

1. Eisenberg RL: Gastrointestinal Radiology: A Pattern Approach. (ed 3) Philadelphia: Lippincott, 1996, pp 493-506.

\section{Gamut G-49-S1 \\ BENIGN TUMORS OF THE SMALL BOWEL}

1. Adenoma

2. Carcinoid

3. Fibroma

4. Gastrointestinal stromal tumor ${ }_{\mathrm{g}}$ (esp. leiomyoma; neurofibroma)

5. Hamartoma (Peutz-Jeghers S.)

6. Hemangioma; lymphangioma

7. Lipoma

\section{References}

1. Eisenberg RL: Gastrointestinal Radiology: A Pattern Approach. (ed 3) Philadelphia: Lippincott, 1996, pp 493-506

2. Good CA: Tumors of the small intestine. AJR 1963; 89:685-705
Gamut G-49-S2

\section{MALIGNANT TUMORS OF THE SMALL} BOWEL

1. Adenocarcinoma

2. Carcinoid

3. Gastrointestinal stromal tumor (esp. leiomyosarcoma)

4. Kaposi sarcoma

5. Lymphoma

6. Metastasis (esp. melanoma)

\section{References}

1. Eisenberg RL: Gastrointestinal Radiology: A Pattern Approach. (ed 3) Philadelphia: Lippincott, 1996, pp 493-506

2. Good CA: Tumors of the small intestine. AJR 1963; 89: 685-705

3. Maglinte DDT, Kelvin FM, Herlinger H: Malignant tumors of the small bowel. In: Gore RM, Levine MS: Textbook of Gastrointestinal Radiology. (ed 2) Philadelphia: WB Saunders, 2000, pp 792-814

\section{Gamut G-50 \\ MULTIPLE INTRALUMINAL, MUCOSAL, OR INTRAMURAL FILLING DEFECTS IN THE SMALL BOWEL}

\section{COMMON}

1. Brunner's gland hyperplasia (duodenum)

2. Food particles; seeds; foreign bodies; pills

3. Meconium ileus (cystic fibrosis (mucoviscidosis)

4. Metastases (esp. melanoma; carcinoma of breast, lung, or ovary; Kaposi sarcoma)

5. Nodular lymphoid hyperplasia (esp. in dysgammaglobulinemia)

6. Parasites (ascarids; tapeworms-Taenia saginata)

7. Polyposis syndromes (esp. Peutz-Jeghers S.; Gardner S.; Cronkhite-Canada S.,) (See G-106) 


\section{UNCOMMON}

1. Amyloidosis

2. Behçet $\mathrm{S}$.

3. Benign neoplasms (eg, hemangiomas; lipomas; leiomyomas, neurofibromas and other gastrointestinal stromal tumors ${ }_{\mathrm{g}}$ )

4. Blood clots

5. Carcinoids

6. Crohn's disease (cobblestone pattern)

7. Gallstones

8. Hyperplastic Peyer's patches in ileum (typhoid fever)

9. Lymphoma

10. Mastocytosis (duodenum)

11. Varices

\section{References}

1. Eisenberg RL: Gastrointestinal Imaging: A Pattern Approach (ed 3). Philadelphia: Lippincott, 1996, pp 507-517

2. Rubesin SE, Herlinger H: Small bowel: Differential diagnosis. In: Gore RM, Levine MS: Textbook of Gastrointestinal Radiology. (ed 2) Philadelphia: WB Saunders, 2000, pp 884-891

\section{Gamut G-51-1}

\section{SMALL BOWEL DIVERTICULUM}

\section{COMMON}

1. Duodenal diverticulum

\section{UNCOMMON}

1. Diverticulosis of small bowel (esp. jejunal diverticulosis)

2. Giant duodenal diverticulum

3. Jejunal diverticulum

4. Ileal diverticulum

5. Meckel's diverticulum

\section{Reference}

1. Eisenberg RL: Gastrointestinal Radiology: A Pattern Approach. (ed 3) Philadelphia: Lippincott, 1996, pp 541-551

\section{Gamut G-51-2 \\ SMALL BOWEL PSEUDODIVERTICULUM}

\section{COMMON \\ 1. Chronic duodenal ulcer disease with pseudo- diverticular outpouchings \\ 2. Crohn's disease (pseudodiverticula; fistulae)}

\section{UNCOMMON}

1. Communicating ileal duplication

2. [Giant duodenal ulcer]

3. Intraluminal duodenal diverticulum

4. [Lymphoma ("aneurysmal" dilatation)]

5. Scleroderma

[ ] This condition does not actually cause the gamuted imaging finding, but can produce imaging changes that simulate it.

\section{Reference}

1. Eisenberg RL: Gastrointestinal Radiology: A Pattern Approach. (ed 3) Philadelphia: Lippincott, 1996, pp 541-551
THICKENING OF THE SMALL BOWEL WALL, GENERALIZED OR LOCALIZED (BARIUM, US, CT, MRI)

\section{COMMON}

1. Crohn's disease

2. Edema (eg, heart failure; constrictive pericarditis; angioneurotic edema; portal hypertension)

3. Eosinophilic enteritis; amyloidosis (fold thickening may be regular in early stages of these diseases)

4. Hemorrhage, intramural (eg, trauma; anticoagulant therapy; hemophilia; other bleeding or clotting disorder $_{\mathrm{g}}$; vasculitis; Henoch-Schönlein purpura) 
5. Hypoproteinemia (eg, cirrhosis; Budd-Chiari S.; nephrosis; malnutrition; burn; dysproteinemia)

6. Intestinal lymphangiectasia, primary or secondary (eg, mesenteric neoplasm)

7. Ischemic bowel disease or infarction (eg, atherosclerosis; thromboembolism; vasculitis; polyarteritis nodosa; hypotension)

8. Metastatic disease (esp. malignant melanoma; carcinoma of breast, lung or ovary; Kaposi sarcoma)

9. Neoplasm, primary (eg, carcinoma; carcinoid; lipoma; gastrointestinal stromal tumor $_{\mathrm{g}}$ leiomyoma; leiomyosarcoma)

10. Opportunistic infection, esp, in AIDS (eg, Cryptosporidium; Campylobacter fetus [jejuni]; Candida; Mycobacterium avium-intracellulare; cytomegalovirus)

11. Parasitic disease (giardiasis; strongyloidiasis; intestinal capillariasis; hookworm disease; schistosomiasis); terminal ileum—amebiasis; anisakiasis; angiostrongyliasis costaricensis

12. Peptic ulcer; Zollinger-Ellison S.; multiple endocrine neoplasia (MEN) S. (See J-5)

\section{UNCOMMON}

1. A-beta-lipoproteinemia

2. Alpha chain disease

3. Amyloidosis

4. Behçet disease

5. Cystic fibrosis (mucoviscidosis)

6. Enterocolitis (eg, typhoid fever; Yersinia)

7. Eosinophilic enteritis

8. Graft-versus-host disease

9. Infections, other (eg, E. coli; Vibrio; histoplasmosis)

10. Interloop abscess

11. Intestinal lymphangiectasia, primary or secondary (eg, mesenteric neoplasm)

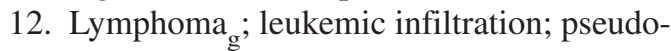
lymphoma

13. Mastocytosis

14. Menetrier's disease (stomach and duodenum)

15. Pancreatitis

16. Radiation enteropathy, acute or chronic

17. Tuberculous enteritis or peritonitis
18. Waldenström's macroglobulinemia

19. Whipple's disease

20. Xanthomatosis

\section{References}

1. Berk RN, Wall SD, McArdle CB, et al: Cryptosporidiosis of the stomach and small intestine in patients with AIDS. AJR 1984;143:549-554

2. Chen MYM, Ott DJ, Gelfand DW: Radiologic interpretation of diffuse small bowel diseases. Appl Radiol Sept 1990; 30-37

3. Eisenberg RL: Gastrointestinal Radiology: A Pattern Approach (ed 3). Philadelphia: Lippincott, 1996, pp 478-492

4. Goldberg HI, Sheft DJ: Abnormalities in small intestine contour and caliber. Radiol Clin North Am 1976;14: 461-475

5. Halpert RD, Feczko PJ: Gastrointestinal Radiology: The Requisites. (ed 2) St. Louis: Mosby-Year Book, 1999

6. Olmsted WW, Reagin DE: Pathophysiology of enlargment of the small bowel fold. AJR 1976;127:423-428

7. Rubesin SE, Herlinger H: Small bowel: Differential diagnosis. In: Gore RM, Levine MS: Textbook of Gastrointestinal Radiology. (ed 2) Philadelphia: WB Saunders, 2000, pp 884-891

\section{Gamut G-53}

\section{MUCOSAL DESTRUCTION OF THE SMALL BOWEL WITH OR WITHOUT STRICTURE (LOCAL OR WIDESPREAD)}

\section{COMMON}

*1. Crohn's disease; other nonspecific enteritis

*2. Lymphoma $_{\mathrm{g}}$

\section{UNCOMMON}

1. [Abscess, interloop]

2. [Adhesions]

*3. Amyloidosis

4. Carcinoid

5. Carcinoma

*6. Eosinophilic enteritis

(continued) 
7. Fungus disease ${ }_{\mathrm{g}}$ (eg, histoplasmosis; actinomycosis)

*8. Mastocytosis

*9. Metastatic disease (esp. from melanoma; carcinoma of breast, lung, ovary, uterus, pancreas, or GI tract)

10. [Pancreatitis]

*11. Parasitic disease (giardiasis; strongyloidiasis; intestinal capillariasis; hookworm disease; schistosomiasis; also amebiasis, anisakiasis, angiostrongyliasis costaricensis in terminal ileum)

12. Potassium enteritis

*13. Radiation enteritis

14. Sarcoma (eg, leiomyosarcoma; Kaposi sarcoma)

*15. Scleroderma

*16. Tuberculosis

17. Typhoid fever

18. Ulcerative colitis ("backwash ileitis")

19. Vascular occlusion; ischemia

20. Yersinia enterocolitis

* May be widespread.

[ ] This condition does not actually cause the gamuted imaging finding, but can produce imaging changes that simulate it.

\section{References}

1. Gelfand DW: Gastrointestinal Radiology. New York: Churchill Livingstone, 1984, p 284

2. Papadopoulos VD, Nolan DJ: Carcinoma of the small intestine. Clin Radiol 1985;36:409 413

3. Palmer PES, Reeder MM: The Imaging of Tropical Diseases. Heidelberg: Springer-Verlag, 2001

\section{Gamut G-54}

\section{REGULAR THICKENING OF SMALL BOWEL FOLDS ( $>3 \mathrm{~mm}$ )}

1. Angioneurotic edema

2. Eosinophilic enteritis; amyloidosis (fold thickening may be regular in early stages of these diseases)

3. Heart failure; constrictive pericarditis
4. Hypoproteinemia (eg, cirrhosis; Budd-Chiari S.; nephrosis; malnutrition; burn; dysproteinemia; A-beta-lipoproteinemia)

5. Hemorrhage, intramural (eg, trauma; anticoagulant therapy; hemophilia; other bleeding or clotting disorder $_{\mathrm{g}}$; vasculitis; Henoch-Schönlein purpura)

6. Infection (eg, giardiasis; typhoid fever)

7. Intestinal lymphangiectasia, primary or secondary (eg, mesenteric neoplasm)

8. Ischemic bowel disease or infarction (eg, atherosclerosis; thromboembolism; vasculitis; polyarteritis nodosa; hypotension)

9. Radiation enteropathy

10. Xanthomatosis

\section{References}

1. Eisenberg RL: Gastrointestinal Radiology: A Pattern Approach (ed 3). Philadelphia: Lippincott, 1996, pp 468477

2. Goldberg HI, Sheft DJ: Abnormalities in small intestine contour and caliber. Radiol Clin North Am 1976;14: 461-475

3. Olmsted WW, Reagin DE: Pathophysiology of enlargement of the small bowel fold. AJR 1976;127:423-428

4. Rubesin SE, Herlinger H: Small bowel: Differential diagnosis. In: Gore RM, Levine MS: Textbook of Gastrointestinal Radiology. (ed 2) Philadelphia: WB Saunders, 2000, pp 884-891

\section{Gamut G-55}

\section{GENERALIZED IRREGULAR OR DISTORTED SMALL BOWEL FOLDS}

\section{COMMON}

1. Crohn's disease

2. Opportunistic infection, esp, in AIDS (eg, Cryptosporidium, Candida, Mycobacterium aviumintercellulare, cytomegalovirus)

3. Parasitic disease (giardiasis; strongyloidiasis; intestinal capillariasis; hookworm disease; schistosomiasis); terminal ileum-amebiasis; anisakiasis; angiostrongyliasis costaricensis 
4. Peptic ulcer; Zollinger-Ellison S.; multiple endocrine neoplasia (MEN) S. (See J-5)

\section{UNCOMMON}

1. A-beta-lipoproteinemia

2. Alpha chain disease

3. Amyloidosis

4. Cystic fibrosis (mucoviscidosis)

5. Enterocolitis (eg, typhoid fever; Yersinia)

6. Eosinophilic enteritis

7. Infections, other (eg, Campylobacter fetus [jejuni]; Shigella; E. coli; Vibrio; histoplasmosis)

8. Interloop abscess

9. Intestinal lymphangiectasia, primary or secondary (eg, mesenteric neoplasm)

10. Lymphoma ${ }_{\mathrm{g}}$; pseudolymphoma

11. Mastocytosis

12. Ménétrier's disease (stomach and duodenum)

13. Pancreatitis

14. Radiation injury, acute or chronic

15. Tuberculous enteritis or peritonitis

16. Waldenström's macroglobulinemia

17. Whipple's disease

\section{References}

1. Berk RN, Wall SD, McArdle CB, et al: Cryptosporidiosis of the stomach and small intestine in patients with AIDS. AJR 1984;143:549-554

2. Chen MYM, Ott DJ, Gelfand DW: Radiologic interpretation of diffuse small bowel diseases. Appl Radiol Sept 1990;30-37

3. Eisenberg RL: Gastrointestinal Radiology: A Pattern Approach (ed 3). Philadelphia: Lippincott, 1996, pp 478-492

4. Goldberg HI, Sheft DJ: Abnormalities in small intestine contour and caliber. Radiol Clin North Am 1976;14: 461-475

5. Olmsted WW, Reagin DE: Pathophysiology of enlargment of the small bowel fold. AJR 1976;127:423-428

6. Palmer PES, Reeder MM: The Imaging of Tropical Diseases. Heidelberg: Springer-Verlag, 2001

\section{Gamut G-56}

\section{SIMULTANEOUS FOLD THICKENING OF THE STOMACH AND SMALL BOWEL}

\section{COMMON}

1. Crohn's disease

2. Zollinger-Ellison S.; peptic ulcer disease

\section{UNCOMMON}

1. Amyloidosis

2. Eosinophilic gastroenteritis

3. Gastric varices with hypoproteinemia

4. Lymphoma ${ }_{\mathrm{g}}$; pseudolymphoma

5. Ménétrier's disease

6. Opportunistic infection, esp, in AIDS (eg, Cryptosporidium; Candida; Mycobacterium aviumintercellulare; cytomegalovirus)

7. Pancreatitis

8. Parasitic disease (eg, strongyloidiasis; schistosomiasis; anisakiasis)

9. Tuberculosis

10. Whipple's disease

\section{Reference}

1. Eisenberg RL: Clinical Imaging: An Atlas of Differential Diagnosis. (ed 3) Philadelphia: Lippincott-Raven, 1997, pp 384-385

\section{Gamut G-57}

\section{MALABSORPTION PATTERN IN THE SMALL BOWEL}

\section{COMMON}

1. Blind loop S. (See G-58)

2. Crohn's disease

3. Cystic fibrosis (mucoviscidosis)

4. Pancreatic disease (insufficiency; chronic pancreatitis; carcinoma; gastrinoma) 
5. Parasitic disease (giardiasis; hookworm disease; strongyloidiasis; schistosomiasis japonica; intestinal capillariasis)

6. Postoperative (eg, postgastrectomy steatorrhea; gastroileostomy; short bowel S.; pancreatectomy)

7. Sprue, tropical or nontropical (celiac disease)

8. Steatorrhea, idiopathic

\section{UNCOMMON}

1. A-beta-lipoproteinemia

2. Acrodermatitis enteropathica

3. Acute bacterial infection

4. AIDS

5. Allergy (eg, angioneurotic edema)

6. Amyloidosis

7. Bile duct obstruction

8. Carcinoid syndrome

9. Chronic granulomatous disease of childhood

10. Cronkhite-Canada S.

11. Diabetes

12. Disaccharidosis (eg, lactase deficiency)

13. Drug therapy (eg, antimetabolites)

14. Ehlers-Danlos S.

15. Emotional states; anorexia nervosa

16. Eosinophilic gastroenteritis

17. Fistula (See G-107)

18. Henoch-Schönlein purpura

19. Hepatobiliary disease (eg, biliary cirrhosis; biliary atresia)

20. Hypoparathyroidism

21. Hypopituitarism

22. Hypothyroidism (cretinism); hyperthyroidism

23. Immunologic disorder ${ }_{g}$

24. Intestinal lymphangiectasia

25. Ischemia of intestine, chronic

26. Jejunal diverticulosis

27. Johanson-Blizzard S.

28. Lymphoma

29. Mastocytosis

30. Metastases, peritoneal

31. Multiple endocrine neoplasia (MEN) S. (eg, gastrinoma; pancreatic non-beta islet cell tumor (VIPoma) or hyperplasia) (See J-5)
32. Nephrotic syndrome

33. Nutritional deficiency (kwashiorkor; pellagra)

34. Protein-losing enteropathy

35. Radiation gastroenteritis

36. Scleroderma; dermatomyositis

37. Tuberculous peritonitis

38. Waldenström's macroglobulinemia; alpha chain disease

39. Whipple's disease

40. Wolman's disease (familial xanthomatosis)

41. Zollinger-Ellison S.

\section{References}

1. Herlinger H: Malabsorption. In: Gore RM, Levine MS: Textbook of Gastrointestinal Radiology. (ed 2) Philadelphia: WB Saunders, 2000, pp 759-784

2. Meyer B, Cerda JJ: Malabsorption: Pathophysiologic considerations and diagnosis. Compr Ther 1985;11:49-54

3. Riley SA, Marsh MN: Maldigestion and malabsorption. In: Feldman M, Scharschmidt BF, Sleisenger MH: Gastrointestinal and Liver Disease. (ed 6) Philadelphia: WB Saunders, 2000, pp 1501-1522

4. Taybi H, Lachman RS: Radiology of Syndromes, Metabolic Disorders, and Skeletal Dysplasias. (ed 4) St. Louis: MosbyYear Book, 1996, pp 963-964

5. Teplick JG, Haskin ME: Roentgenologic Diagnosis. (ed 3) Philadelphia: WB Saunders, 1976

\section{Gamut G-58}

\section{BLIND LOOP SYNDROME}

\section{COMMON}

1. Postoperative (eg, side-to-side anastomosis; gastroileostomy; bypass procedure; short bowel syndrome)

2. Small bowel stricture with proximal dilatation

\section{UNCOMMON}

1. Diverticulosis of small bowel (esp. jejunum)

2. Duplication of intestine

3. Meckel's diverticulum, large 
4. Sluggish transit (eg, myxedema; cretinism; scleroderma)

\section{Reference}

1. Challacombe DN, Richardson JM, Edkins S, et al: Ileal blind loop in childhood. Am J Dis Child 1974;123:719-723

\section{Gamut G-59}

\section{SMALL BOWEL DILATATION WITH THICKENED MUCOSAL FOLD PATTERN}

\section{COMMON}

1. Crohn's disease

2. Infectious enteritis, esp. in AIDS (eg, cryptosporidiosis; cytomegalovirus; Candida; Salmonella; Mycobacterium avium-intracellulare)

3. Mesenteric infarction (venous or embolic arterial) or advanced ischemia (atherosclerosis)

4. Metastatic disease to bowel wall or mesentery

5. Zollinger-Ellison S.

\section{UNCOMMON}

1. A-beta-lipoproteinemia; A-alpha-lipoproteinemia

2. Amyloidosis

3. Compensatory dilatation of remaining bowel after extensive small bowel resection

4. Hypoalbuminemia (eg, cirrhosis; nephrotic S.)

5. Lymphoma $_{\mathrm{g}}$

6. Parasitic disease (eg, thickened folds with malabsorption and dilated loops can be seen in strongyloidiasis, hookworm disease, intestinal capillariasis, and schistosomiasis japonica)

7. Radiation enteritis

8. Tropical sprue

9. Tuberculosis

\section{Reference}

1. Eisenberg RL: Gastrointestinal Radiology: A Pattern Approach. (ed 3) Philadelphia: Lippincott, 1996, pp 464-467

\section{Gamut G-60}

\section{SMALL BOWEL DILATATION WITH NORMAL FOLD PATTERN}

\section{COMMON}

1. Adynamic ("paralytic") ileus

2. Drug effect (esp. anticholinergics)

3. Mechanical obstruction

4. Mesenteric ischemia (eg, atherosclerosis; lupus erythematosus)

5. Postvagotomy; gastrectomy (dumping syndrome)

6. Sprue (esp. nontropical); celiac disease; other malabsorption syndromes (See G-57)

\section{UNCOMMON}

1. Amyloidosis

2. Chagas' disease

3. Chronic idiopathic intestinal pseudo-obstruction

4. Hypokalemia (esp. in diabetic)

5. Lactase deficiency

6. Scleroderma; dermatomyositis

\section{References}

1. Chen MYM, Ott DJ, Gelfand DW: Radiologic interpretation of diffuse small bowel diseases. Appl Radiol Sept 1990; 30-37

2. Eisenberg RL: Gastrointestinal Radiology: A Pattern Approach. (ed 3) Philadelphia: Lippincott, 1996, pp 453-463

\section{Gamut G-61 \\ ACUTE NONOBSTRUCTIVE SMALL BOWEL DISTENTION (“PARALYTIC ILEUS”)}

\section{COMMON}

1. Drug effect (eg, atropine; morphine; barbiturates; Lomotil; Pro-Banthine; hexamethonium; L-dopa)

2. Electrolyte imbalance (eg, hypokalemia; hypochloremia; calcium or magnesium abnormality)

(continued) 
3. Gastroenteritis, acute; food poisoning

4. Pain in abdomen (eg, renal, ureteral, or common bile duct stone; torsion of uterine fibroid or ovarian cyst or tumor; lead colic; sickle cell crisis; tabetic crisis; porphyria)

5. Peritonitis, acute

6. Pneumonia; other acute thoracic disease (eg, myocardial infarction; heart failure)

7. Postoperative (abdominal or pelvic surgery)

8. Retroperitoneal hemorrhage

9. "Sentinel loop," localized ileus (eg, acute cholecystitis; appendicitis; acute pancreatitis; acute diverticulitis) (See G-80)

10. Shock; gram-negative septicemia; hypoxia

11. Trauma (esp. spine or lower rib; abdominal contusion; intramural hematoma)

12. Vascular occlusion (eg, mesenteric infarction)

\section{UNCOMMON}

1. Adrenal insufficiency

2. Aerophagia; assisted respiration

3. Ceroidosis (malabsorption with prolonged vitamin E depletion)

4. Chronic idiopathic intestinal pseudo-obstruction

5. Diabetic acidosis; insulin shock

6. Enterocolitis (eg, typhoid fever; Yersinia)

7. Hypoparathyroidism

8. Hypothyroidism

9. Interloop abscess

10. Myotonic dystrophy; muscular dystrophy

11. Neonatal adynamic ileus (eg, septicemia; hypoxiainduced vasculitis; infantile respiratory distress S.; intestinal infection; peritonitis; mesenteric thrombosis)

12. Neonatal necrotizing enterocolitis

13. Renal failure; uremia; acute glomerulonephritis

14. Urinary retention

\section{References}

1. Burgener FA, Kormano M: Differential Diagnosis in Conventional Radiology. (ed 2) New York: Thieme Medical Publ, 1991, pp 601-602

2. Eisenberg RL: Gastrointestinal Radiology: A Pattern Approach. (ed 3) Philadelphia: Lippincott, 1996, pp 442-452

\section{Gamut G-62}

\section{CHRONIC NONOBSTRUCTIVE SMALL BOWEL DISTENTION}

\section{COMMON}

1. Ascites

2. Connective tissue disease (collagen disease) $)_{\mathrm{g}}$ (esp. scleroderma)

3. Neurologic or muscular disorder ${ }_{\mathrm{g}}$ (eg, parkinsonism; myotonic dystrophy; tabes; spinal cord lesion)

4. Sprue, tropical or nontropical (celiac disease)

5. Vagotomy

\section{UNCOMMON}

1. Adrenal insufficiency

2. Allergic enterocolitis

3. Amyloidosis

4. Ceroidosis (prolonged malabsorption and vitamin E depletion)

5. Chronic idiopathic intestinal pseudo-obstruction

6. Congenital short intestine

7. Cystic fibrosis (mucoviscidosis)

8. Diabetes with hypokalemia

9. Disaccharidase deficiency (eg, lactase deficiency)

10. Drug effect (eg, Lomotil; morphine)

11. Eosinophilic gastroenteritis

12. Hypoparathyroidism

13. Hypoproteinemia (eg, cirrhosis; nephrosis; malnutrition; burn; A-beta-lipoproteinemia)

14. Intestinal lymphangiectasia

15. Jejunal diverticulosis

16. Lymphoma

17. Malrotation; internal hernia

18. Mesenteritis

19. Myxedema; hypothyroidism

20. Parasitic disease (esp. Chagas' disease; chronic strongyloidiasis; schistosomiasis japonica)

21. Postoperative (eg, gastrectomy; colectomy; intestinal bypass)

22. Renal failure; uremia; peritoneal dialysis

23. Vascular insufficiency (mesenteric ischemia); vasculitis (eg, lupus erythematosus; polyarteritis nodosa) 
24. Waldenström's macroglobulinemia

25. Whipple's disease

\section{References}

1. Eisenberg RL: Clinical Imaging: An Atlas of Differential Diagnosis. (ed 3) Philadelphia: Lippincott-Raven, 1997 , pp 358-361

2. Rubesin SE, Herlinger H: Small bowel: Differential diagnosis. In: Gore RM, Levine MS: Textbook of Gastrointestinal Radiology. (ed 2) Philadelphia: WB Saunders, 2000, pp 884-891

3. Seaman WB: Motor dysfunction of the gastrointestinal tract. AJR 1972;116:235-244

\section{Gamut G-63}

\section{TERMINAL ILEUM LESION}

\section{COMMON}

1. Appendicitis

2. Carcinoid

3. Crohn's disease

4. Intussusception

5. Mass, extrinsic (eg, ovarian or other pelvic neoplasm; aneurysm of iliac artery)

6. Meconium ileus (cystic fibrosis \{mucoviscidosis

7. Nodular lymphoid hyperplasia; normal lymphoid follicles

\section{UNCOMMON}

1. Diverticulitis

2. Endometrial implant

3. Food particles; foreign body; gallstone

4. Fungus disease (eg, actinomycosis; histoplasmosis)

5. Intramural hematoma

6. Laxative abuse

7. Meckel's diverticulum

8. Mesenteric infarction; ischemic enteritis

9. Metastasis (esp. from gastric, colonic or ovarian neoplasm)

10. Neoplasm, benign or malignant (eg, gastrointestinal stromal tumor ${ }_{\mathrm{g}}$; carcinoma; sarcoma; lymphoma $_{\mathrm{g}}$ )
11. Parasitic disease

a. Intraluminal worms (eg, Ascaris; tapewormTaenia saginata)

b. Inflammatory changes (eg, schistosomiasis; amebiasis; strongyloidiasis; rarely giardiasis; intestinal capillariasis; anisakiasis; angiostrongyliasis costaricensis)

12. Polyp $_{\mathrm{g}}$ (See G-106)

13. Radiation enteritis

14. Tuberculosis

15. Typhoid fever

16. Ulcerative colitis ("backwash ileitis")

17. Yersinia enterocolitis

\section{References}

1. Calenoff L: Rare ileocecal lesions. AJR 1970;110:343-351

2. Gelfand DW: Gastrointestinal Radiology. New York: Churchill Livingstone, 1984, p 335

3. Herlinger H, Ekberg OT: Other inflammatory conditions of the small bowel. In: Gore RM, Levine MS: Textbook of Gastrointestinal Radiology. (ed 2) Philadelphia: WB Saunders, 2000, pp 704-725

4. Jeffree MA, et al: Primary carcinoid tumors of the ileum: the radiological appearances. Clin Radiol 1984;35:451-455

5. Marshak RH, Lindner AE, Maklansky D: Radiology of the Colon. Philadelphia: WB Saunders, 1980

6. Williams RH, Dixit JK: Filling defect in terminal ileum due to an aneurysm of right common iliac artery. South Med J $1975 ; 68: 783-785$

\section{Gamut G-64-S}

\section{NONDIAPHRAGMATIC HERNIAS}

\section{COMMON}

1. Femoral

2. Incisional

3. Inguinal

4. Umbilical (incl. omphalocele)

5. Ventral

\section{UNCOMMON}

1. Lesser sac (foramen of Winslow)

2. Lumbar

(continued) 
3. Mesenteric (small bowel; sigmoid)

4. Obturator

5. Paracecal

6. Paraduodenal

7. Pelvic (broad ligament)

8. Perineal

9. Retroperitoneal

10. Sciatic

11. Spigelian

\section{Reference}

1. Eisenberg RL: Gastrointestinal Radiology: A Pattern Approach. (ed 3) Philadelphia: Lippincott, 1996, pp 909-924

\section{Gamut G-65}

\section{ABNORMALITIES OF BOWEL ROTATION}

\section{COMMON}

1. Malrotation

2. Midgut volvulus with malrotation

3. Mobile cecum (high or midline)

4. Nonrotation

\section{UNCOMMON}

1. Exomphalos

2. Extroversion of cloaca

3. Paraduodenal hernia

4. Reverse rotation

\section{References}

1. Gaines PA, Saunders AJ, Drake D: Midgut malrotation diagnosed by ultrasound. 1987; 38:51-53

2. Houston CS, Wittenborg MH: Roentgen evaluation of anomalies of rotation and fixation of the bowel in children. Radiology 1965;84:1-17

3. Javors BR: Applied embryology of the gastrointestinal tract. In: Gore RM, Levine MS: Textbook of Gastrointestinal Radiology. (ed 2) Philadelphia: WB Saunders, 2000, p 2020

\section{Gamut G-66}

\section{CONGENITAL SYNDROMES ASSOCIATED WITH INTESTINAL MALROTATION}

1. Abdominal heterotaxy

2. Asplenia or polysplenia $S$.

3. Brachmann-de Lange $S$.

4. Cantrell S.

5. Coffin-Siris $S$.

6. FG syndrome

7. Mobile cecum S.

8. Prune-belly S. (Eagle-Barrett S.)

9. Trisomy $13 \mathrm{~S}$.

10. Trisomy $18 \mathrm{~S}$.

11. Trisomy 21 S. (Down S.)

\section{References}

1. Fernbach SK: Neonatal gastrointestinal radiology. In: Gore RM, Levine MS: Textbook of Gastrointestinal Radiology. (ed 2) Philadelphia: WB Saunders, 2000, pp 2042-2074

2. Jones KL: Smith's Recognizable Patterns of Human Malformation. Philadelphia: WB Saunders, 1988

3. Taybi H, Lachman RS: Radiology of Syndromes, Metabolic Disorders, and Skeletal Dysplasias. (ed 4) St. Louis: MosbyYear Book, 1996, p 964

\section{Gamut G-67}

\section{APHTHOID ULCERS IN THE SMALL BOWEL OR COLON*}

\section{COMMON}

1. Crohn's disease

\section{UNCOMMON}

1. Amebiasis

2. [Artifacts (eg, fecal debris; flocculated barium; innominate grooves of colon)]

3. Behçet $\mathrm{S}$. 

4. Candidiasis
5. Cytomegalovirus infection of ileum and colon (also esophagus and stomach)
6. Ischemic colitis
7. [Lymphoid hyperplasia of colon]
8. Salmonellosis
9. Tuberculosis
10. Yersinia enterocolitis

* Tiny discrete central ulcer containing barium surrounded by a halo of edematous mucosa, best seen on air-contrast examination.

[ ] This condition does not actually cause the gamuted imaging finding, but can produce imaging changes that simulate it.

\section{References}

1. Eisenberg RL: Gastrointestinal Radiology: A Pattern Approach. (ed 3) Philadelphia: Lippincott, 1996

2. Gedgaudas-McClees EK: Aphthoid ulcerations in ileocecal candidiasis. AJR 1983; 141:973-977

3. Gore RM: Inflammatory disease. In: Margulis AR: Modern Imaging of the Alimentary Tube. New York: SpringerVerlag, 1998, pp 185-216

4. Simpkins KC: Aphthoid ulcers in Crohn's colitis. Clin Radiol 1977;28:601-608

\section{Gamut G-68}

\section{INNUMERABLE TINY NODULES (SAND-LIKE OR GRANULAR LUCENCIES SMALLER THAN 5 MM) IN THE SMALL BOWEL OR COLON}

\section{COMMON}

1. Crohn's disease ("cobblestone" pattern)

2. [Food particles; seeds; air bubbles]

3. Nodular lymphoid hyperplasia (eg, dysgammaglobulinemia)

4. Normal lymphoid follicles

5. Polyposis syndromes (esp. Cronkhite-Canada S.) (See G-106)

\section{UNCOMMON}

1. A-beta-lipoproteinemia

2. Amyloidosis
3. Cystic fibrosis (mucoviscidosis)

4. Eosinophilic enteritis

5. Histoplasmosis

6. Hyperplastic Peyer's patches (eg, typhoid fever)

7. Intestinal lymphangiectasia

8. Lymphoma $_{\mathrm{g}}$

9. Mastocytosis

10. Mycobacterial enteritis

11. Ulcerative colitis

12. Waldenström's macroglobulinemia; heavy chain disease

13. Whipple's disease

14. Yersinia enterocolitis

[ ] This condition does not actually cause the gamuted imaging finding, but can produce imaging changes that simulate it.

\section{References}

1. Eisenberg RL: Gastrointestinal Radiology: A Pattern Approach. (ed 3) Philadelphia: Lippincott, 1996, pp 518-524

2. Kenney PJ, Koehler RE, Shackelford GD: The clinical significance of large lymphoid follicles of the colon. Radiology 1982;142:41-46

3. Reeder MM: RPC of the Month from the AFIP: Nodular lymphoid hyperplasia of the small intestine. Radiology 1969:93:427-433

4. Rubesin SE, Herlinger H: Small bowel: Differential diagnosis. In: Gore RM, Levine MS: Textbook of Gastrointestinal Radiology. (ed 2) Philadelphia: WB Saunders, 2000, pp 884-890

\section{Gamut G-69 \\ MESENTERIC VASCULAR COMPROMISE (INTESTINAL ISCHEMIA OR INFARCTION)}

\section{COMMON}

1. Arterial thromboembolism (eg, secondary to myocardial infarction; rheumatic heart disease; atrial fibrillation)

2. Arteriosclerosis

3. Digitalis toxicity 
4. Heart failure

5. Iatrogenic (eg, catheter arteriography; drug instillation)

6. Idiopathic (normal vessels)

7. Intestinal obstruction (esp. strangulation)

8. Peritoneal band or adhesion

9. Septicemia (eg, drug abuse; bacterial endocarditis)

10. Vascular compression by extrinsic mass

11. Venous thrombosis

\section{UNCOMMON}

1. Abdominal or pelvic inflammatory disease

2. Arteritis (eg, Takayasu's arteritis; polyarteritis nodosa)

3. Coarctation of aorta (esp. postoperative)

4. Dissecting aneurysm

5. Fibromuscular hyperplasia of mesenteric artery

6. Polycythemia

7. Postoperative (esp. surgical ligation)

8. Radiation injury

9. Transient ischemia of children

10. Trauma to the intestine or its vessels

\section{References}

1. Kaufman SL, Harrington DP, Siegelman SS: Superior mesenteric artery embolization: An angiography emergency. Radiology 1977;124:625-630

2. Smith SL, Tutton RH, Ochsner SF: Roentgenographic aspects of intestinal ischemia. AJR 1972;116:249-255

3. Szucs RA, Wolf EL, Gramm HF, et al: Miscellaneous abnormalities of the colon. In: Gore RM, Levine MS: Textbook of Gastrointestinal Radiology. (ed 2) Philadelphia: WB Saunders, 2000, pp 1084-1122

\section{Gamut G-70 \\ GAS IN THE BOWEL WALL (PNEUMATOSIS INTESTINALIS)}

\section{COMMON}

1. Colitis (eg, ulcerative; tuberculous; amebic; Crohn's disease)

2. Necrosis of the intestine

a. Necrotizing enterocolitis (esp. in premature or debilitated infants)

b. Mesenteric thrombosis with infarction

c. Gangrenous, pseudomembranous, or other enterocolitis

d. Strangulated hernia, volvulus, or other intestinal obstruction

e. Primary infection of bowel wall

f. Ingestion of corrosives

3. Primary, idiopathic (pneumatosis cystoides intestinalis)

4. Toxic megacolon (See G-94)

\section{UNCOMMON}

1. Adynamic ileus (esp. postoperative)

2. Air hose injury of rectum ("goose")

3. Colonic irrigation; hydrogen peroxide enema

4. Congenital obstruction (eg, atresia; stenosis; web; diaphragm; imperforate anus; meconium plug)

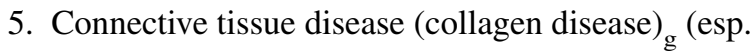
scleroderma)

6. Diabetes with gas-forming organism

7. Graft-versus-host disease (esp. involving cecum)

8. Hirschsprung's disease or other megacolon (See G-93)

9. Iatrogenic (eg, endoscopy; colonoscopy; catheter jejunostomy; umbilical artery catheterization)

10. Idiopathic

11. Leukemia

12. Malabsorption (eg, sprue)

13. Obstructive pulmonary disease, esp. with coughing (eg, emphysema; bullous disease; asthma; chronic bronchitis) 
14. Perforated jejunal diverticulum

15. Pneumomediastinum with abdominal extension of air

16. Postoperative (esp. jejunoileal bypass; postoperative intramural leakage)

17. Pyloroduodenal obstruction (eg, peptic ulcer, esp. with intramural perforation; pyloric stenosis)

18. Steroid and other immunosuppressive therapy

19. Trauma to gut

\section{References}

1. Bryk D: Unusual causes of small-bowel pneumatosis: Perforated duodenal ulcer and perforated jejunal diverticula. Radiology 1973;106:299-302

2. Eisenberg RL: Gastrointestinal Radiology: A Pattern Approach. (ed 3) Philadelphia: Lippincott, 1996, pp 925-937

3. Felson B: Abdominal gas: A roentgen approach. Ann NY Acad Sci 1968;150:141-161

4. Gupta A: Pneumatosis intestinalis in children. Br J Radiol 1978; 51:589-595

5. Keats TE, Smith TH: Benign pneumatosis intestinalis in childhood leukemia. AJR 1974;122;150-152

6. Marshak RH, Lindner AE, Maklansky D: Pneumatosis cystoides coli. Gastrointest Radiol 1977;2:85-89

7. Mueller CF, et al: Pneumatosis intestinalis in collagen disorders. AJR 1972;115:300-305

8. Naggar CZ: Pneumatosis intestinalis following common upper-respiratory-tract infection. JAMA 1976;235;2221-2222

9. Shallal JA, Van Heerden JA, Bartholomew LG, et al: Pneumatosis cystoides intestinalis. Mayo Clin Proc 1974;49: 180-184

10. Strain JD, Rudikoff JC, Moore EE, et al: Pneumatosis intestinalis associated with intracatheter jejunostomy feeding. AJR 1982;139:107-109

\section{Gamut G-71-1}

\section{SMALL AND LARGE BOWEL WALL} THICKENING: HOMOGENEOUS

\section{ATTENUATION ON POSTCONTRAST CT}

\section{COMMON}

1. Carcinoma of small bowel and/or colon

2. Lymphoma

3. Submucosal hemorrhage or hematoma

\section{UNCOMMON}

1. Crohn's disease, chronic

2. Infarcted bowel

3. [Pseudothickening related to incomplete distention and residual fluid]

4. Radiation injury, chronic

[ ] This condition does not actually cause the gamuted imaging finding, but can produce imaging changes that simulate it.

\section{Reference}

1. Macari M, Baltazar EJ: CT of bowel wall thickening: Significance and pitfalls of interpretation. AJR 2001;176: $1105-1116$

Gamut G-71-2

\section{SMALL AND LARGE BOWEL WALL} THICKENING: HETEROGENEOUS (STRATIFIED OR MIXED) ATTENUATION ON POSTCONTRAST CT

\section{STRATIFIED ATTENUATION*}

\section{COMMON}

1. Bowel edema related to cirrhosis or hypoproteinemia

2. Crohn's disease

3. Henoch-Schönlein purpura

4. Infectious enterocolitis

5. Ischemia

6. Lupus erythematosus

7. Radiation injury

8. Ulcerative colitis

9. Vasculitis

\section{UNCOMMON}

1. Infiltrating scirrhous carcinoma (usually colon or rectum)

2. Pneumatosis

3. [Residual fluid and contrast material]

4. Submucosal fat deposition 


\section{MIXED ATTENUATION+}

1. Carcinoma (esp. mucinous adenocarcinoma)

2. Gastrointestinal stromal tumor ${ }_{\mathrm{g}}$

* Alternating (stratified) layers of attenuation in a thickened bowel segment may take the form of a double halo or a target configuration. The double halo sign represents an inner low-attenuation (edema) ring surrounded by an outer higher attenuation ring on postcontrast CT. In the target sign, inner and outer layers of high attenuation surrround a central area of decreased (edema) attenuation. The high attenuation in these signs is related to hyperemia.

+ The grossly thickened bowel wall has several irregular zones of lower attenuation haphazardly located next to areas of higher attenuation. These findings are related to ischemia and necrosis and are seen in highgrade, poorly differentiated neoplasms such as adenocarcinoma and stromal cell tumors.

[ ] This condition does not actually cause the gamuted imaging finding, but can produce imaging changes that simulate it.

\section{Reference}

1. Macari M, Baltazar EJ: CT of bowel wall thickening: Significance and pitfalls of interpretation. AJR 2001;176: $1105-1116$

\section{Gamut G-72-1}

\section{MILD THICKENING ( $<2$ CM) OF BOWEL WALL ON POSTCONTRAST CT}

\section{COMMON}

1. Bowel edema in cirrhosis

2. Crohn's disease

3. Infectious enterocolitis

4. Ischemia

5. Radiation injury

6. Submucosal hemorrhage

7. Ulcerative colitis

\section{UNCOMMON}

1. Adenocarcinoma

2. Lymphoma ${ }_{\mathrm{g}}$

\section{Reference}

1. Macari M, Baltazar EJ: CT of bowel wall thickening: Significance and pitfalls of interpretation. AJR 2001;176: 1105-1116
Gamut G-72-2

\section{MARKED THICKENING ( $>2 \mathrm{CM}$ ) OF BOWEL WALL ON POSTCONTRAST CT}

\section{COMMON}

1. Carcinoma

2. Colitis, severe

3. Gastrointestinal stromal tumor ${ }_{\mathrm{g}}$

4. Lupus erythematosus

5. Lymphoma

6. Metastatic disease involving bowel wall

\section{UNCOMMON}

1. Crohn's disease

2. Cytomegalovirus infection

3. Histoplasmosis

4. Submucosal hemorrhage

5. Tuberculosis

\section{Reference}

1. Macari M, Baltazar EJ: CT of bowel wall thickening: Significance and pitfalls of interpretation. AJR 2001;176: $1105-1116$

\section{Gamut G-73}

\section{SYMMETRIC VERSUS ASYMMETRIC THICKENING OF BOWEL WALL ON POSTCONTRAST CT}

\section{SYMMETRIC}

1. Bowel edema in cirrhosis

2. Crohn's disease

3. Infectious enterocolitis

4. Ischemia

5. Lymphoma

6. Radiation injury

7. Submucosal hemorrhage

8. Ulcerative colitis 


\section{ASYMMETRIC}

1. Carcinoma

2. Gastrointestinal stromal tumor ${ }_{\mathrm{g}}$

\section{Reference}

1. Macari M, Baltazar EJ: CT of bowel wall thickening: Significance and pitfalls of interpretation. AJR 2001;176: 1105-1116

\section{Gamut G-74-1}

\section{FOCAL BOWEL WALL THICKENING (<10 CM) ON POSTCONTRAST CT}

\section{COMMON}

1. Carcinoma (esp. adenocarcinoma)

2. Appendicitis

3. Diverticulitis

\section{UNCOMMON}

1. Crohn's disease

2. Lymphoma

3. Tuberculosis

\section{Reference}

1. Macari M, Baltazar EJ: CT of bowel wall thickening: Significance and pitfalls of interpretation. AJR 2001;176: 1105-1116

\section{Gamut G-74-2}

\section{SEGMENTAL BOWEL WALL THICKENING (10-30 CM) ON POSTCONTRAST CT}

\section{COMMON}

1. Carcinoma (eg, mucinous or colloid carcinoma of colon; scirrhous carcinoma of colon or rectum)

2. Crohn's disease

3. Infectious ileitis

4. Ischemia

5. Lymphoma $_{\mathrm{g}}$

6. Radiation injury

7. Submucosal hemorrhage

\section{UNCOMMON}

1. Lupus erythematosus

\section{Reference}

1. Macari M, Baltazar EJ: CT of bowel wall thickening: Significance and pitfalls of interpretation. AJR 2001;176: 1105-1116

\section{Gamut G-74-3}

\section{DIFFUSE BOWEL WALL THICKENING ON POSTCONTRAST CT}

\section{COMMON}

1. Edema from cirrhosis or hypoproteinemia

2. Infectious enterocolitis

3. Lupus erythematosus

4. Ulcerative colitis 


\section{UNCOMMON}

1. Ischemia

2. Pseudomembranous colitis

\section{Reference}

1. Macari M, Baltazar EJ: CT of bowel wall thickening: Significance and pitfalls of interpretation. AJR 2001;176: 1105-1116

\section{Gamut G-75}

\section{RESIDUAL INTESTINAL BARIUM AFTER GASTROINTESTINAL STUDY (MORE THAN ONE WEEK)}

\section{COMMON}

1. Barium in appendix or diverticula of small bowel or colon

2. Fecal impaction

3. Gastric obstruction

4. Nonobstructive ileus (See G-61)

\section{UNCOMMON}

1. Aganglionosis of colon (eg, Hirschsprung disease; Chagas' disease)

2. Blind loop syndrome (See G-58)

3. [Calcification (esp. milk of calcium)]

4. Cryptosporidiosis

5. Drug effect (eg, morphine)

6. Duplication of intestine

7. Hypothyroidism (eg, myxedema; cretinism)

8. Meckel's diverticulum

9. [Medication, opaque]

10. Perforation of intestine

11. Postoperative (eg, side-to-side anastomosis; gastroileostomy; bypass procedure)

12. Scleroderma

13. Stricture of intestine

[ ] This condition does not actually cause the gamuted imaging finding, but can produce imaging changes that simulate it.

\section{Gamut G-76}

\section{INTESTINAL OBSTRUCTION IN A NEWBORN*}

\section{COMMON}

*1. Congenital stenosis or atresia of stomach, duodenum, small bowel, colon*, rectum*, or anus (imperforate anus)*

2. Hernia, incarcerated, internal or external (eg, inguinal, femoral, umbilical, diaphragmatic, mesenteric defects)

*3. Hirschsprung disease

4. Malrotation with midgut volvulus

*5. Meconium ileus (cystic fibrosis $\{$ mucoviscidosis $\}$ )

*6. Meconium plug S.

*7. Small left colon S.

\section{UNCOMMON}

1. Apple peel intestinal atresia

2. Choledochal cyst

3. Congenital peritoneal bands (Ladd's bands)

4. Inspissated milk S.

5. Intestinal duplication

6. Intraluminal diaphragm or web

7. Intramural hematoma (eg, trauma)

*8. Intussusception (rare in newborn)

9. Meconium peritonitis (eg, meconium ileus)

*10. Megacystis-microcolon-intestinal hypoperistalsis S. (Berdon S.)

11. Neoplasm (usually distention without obstruction)

12. [Paralytic ileus (eg, from drugs given during labor)]

13. Preduodenal portal vein

14. Segmental dilatation of ileum

* Low intestinal obstruction in a newborn.

[ ] This condition does not actually cause the gamuted imaging finding, but can produce imaging changes that simulate it.

\section{References}

1. Carty H, Brereton RJ: The distended neonate. Clin Radiol 1983;34:367-380 
2. Fernbach SK: Neonatal gastrointestinal radiology. In: Gore RM, Levine MS: Textbook of Gastrointestinal Radiology. (ed 2) Philadelphia: WB Saunders, 2000, pp 2042-2074

3. Silverman FN (ed): Caffey's Pediatric X-ray Diagnosis: An Integrated Imaging Approach. (ed 8) Chicago: Year Book Medical Publ, 1985

4. Swischuk LE: Radiology of the Newborn, Infant, and Young Child. (ed 3) Baltimore: Williams \& Wilkins, 1989

5. Taybi H, Lachman RS: Radiology of Syndromes, Metabolic Disorders, and Skeletal Dysplasias. (ed 4) St. Louis: MosbyYear Book, 1996, p 963

\section{Gamut G-77}

\section{INTESTINAL OBSTRUCTION IN A CHILD (See G-76)}

\section{COMMON}

1. Adhesions (inflammatory; postoperative); congenital peritoneal bands (Ladd's bands)

2. Appendicitis (esp. perforated)

3. Hernia, incarcerated (internal or external)

4. Hirschsprung disease

5. Intussusception (eg, ameboma; intestinal duplication; Henoch-Schönlein purpura; idiopathic; Meckel's diverticulum; polyp $_{\mathrm{g}}$; lymphoma $_{\mathrm{g}}$; other neoplasm)

\section{UNCOMMON}

1. Cast S. (cast treatment for scoliosis causing superior mesenteric artery S.)

2. Chronic granulomatous disease of childhood

3. Crohn's disease

4. Cystic fibrosis (mucoviscidosis)

5. Familial Mediterranean fever

6. Fecal impaction

7. Foreign body; bezoar

8. Kawasaki S.

9. Neoplasm, benign or malignant (eg, gastrointestinal stromal tumor ${ }_{\mathrm{g}} ;$ lymphoma $_{\mathrm{g}}$; sarcoma)

10. Neurofibromatosis I (von Recklinghausen disease)

11. Parasitic disease (esp. Ascaris bolus; ameboma)
12. [Pseudo-obstruction, idiopathic]

13. Stenosis, congenital (eg, duodenum; small bowel; rectum)

14. Tuberculous enteritis

15. Volvulus, midgut or other

[ ] This condition does not actually cause the gamuted imaging finding, but can produce imaging changes that simulate it.

\section{References}

1. Silverman FN (ed): Caffey's Pediatric X-ray Diagnosis: An Integrated Imaging Approach. (ed 8) Chicago: Year Book Medical Publ, 1985

2. Swischuk LE, John SD: Differential Diagnosis in Pediatric Radiology. (ed 2) Baltimore: Williams \& Wilkins, 1995

3. Taybi H, Lachman RS: Radiology of Syndromes, Metabolic Disorders, and Skeletal Dysplasias. (ed 4) St. Louis: MosbyYear Book, 1996, p 963

\section{Gamut G-78 \\ INTESTINAL OBSTRUCTION IN AN ADULT}

\section{COMMON}

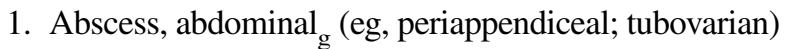

2. Adhesions (inflammatory; postoperative); congenital peritoneal bands (Ladd's bands)

3. Carcinoma of colon, rectum, or rarely, small bowel

4. Crohn's disease

5. Diverticulitis (esp. colonic)

6. Fecal impaction

7. Hernia, incarcerated, internal or external (See G-64-S )

8. Intussusception

9. Metastatic disease (esp. melanoma; carcinoma of ovary, breast, lung; peritoneal carcinomatosis) or invasion of bowel by adjacent pelvic or abdominal malignancy

10. Stricture (eg, neoplastic; inflammatory-lymphogranuloma venereum; radiation; potassium-induced; ischemic; posttraumatic; postoperative)

11. Volvulus (esp. cecal or sigmoid)

(continued) 


\section{UNCOMMON}

1. Amyloidosis

2. Bezoar; enterolith; foreign body

3. Endometriosis

4. Extrinsic pressure from large adjacent neoplasm, distended bladder, or pregnant uterus

5. Familial Mediterranean fever

6. Gallstone ileus

7. Granulomatous disease (eg, actinomycosis; tuberculosis)

8. Hirschsprung disease

9. [Immobilization; Cast S.]

10. Intestinal duplication

11. [Intestinal pseudo-obstruction, idiopathic] (See G-79)

12. Intramural hematoma

13. Lymphoma

14. Neoplasm, other (gastrointestinal stromal tumor ${ }_{\mathrm{g}}$ esp. leiomyoma; carcinoid; sarcoma); cyst

15. Neurofibromatosis I (von Recklinghausen disease)

16. Parasitic disease (Ascaris bolus; amebiasis; Chagas' disease; schistosomiasis)

17. Retractile mesenteritis

18. [Spasm (eg, sickle cell crisis; plumbism; porphyria; tabes; diabetic ketosis; potassium deficiency)]

19. Superior mesenteric artery S.

20. Ulcerative colitis, chronic

21. Vascular occlusion (arterial or venous); mesenteric infarction; ischemic colitis (See G-69)

[ ] This condition does not actually cause the gamuted imaging finding, but can produce imaging changes that simulate it.

\section{References}

1. Eisenberg RL: Gastrointestinal Radiology: A Pattern Approach. (ed 3) Philadelphia: Lippincott, 1996, pp 421-441, 748-767

2. Herlinger H, Rubesin SE, Morris JB: Small bowel obstruction. In: Gore RM, Levine MS: Textbook of Gastrointestinal Radiology. (ed 2) Philadelphia: WB Saunders, 2000, pp 815-837

\section{Gamut G-79}

\section{INTESTINAL PSEUDO-OBSTRUCTION (OGILVIE SYNDROME); BOWEL OBSTRUCTION IN THE ABSENCE OF MECHANICAL BLOCKAGE}

\section{COMMON}

1. Idiopathic ("primary")

2. Paralytic ileus (eg, trauma; hypokalemia; pneumonia; myocardial infarction; pancreatitis; sickle cell crisis)

\section{UNCOMMON}

1. Amyloidosis

2. Celiac disease; sprue

3. Ceroidosis (prolonged malabsorption and vitamin E depletion)

4. Connective tissue disorder (collagen disease)

5. Diverticulosis of small bowel

6. Drug reaction (eg, phenothiazine; antidepressant; anti-Parkinsonism drugs; morphine)

7. Endocrine disorder (eg, myxedema; diabetes; hypoparathyroidism; pheochromocytoma)

8. Enteric muscle disorder (eg, myotonic dystrophy; scleroderma)

9. Jejunoileal bypass

10. Neonatal adynamic ileus

11. Neurologic disorder (eg, parkinsonism, stroke, paralysis, brain damage)

12. Parasitic disease (eg, Chagas' disease; chronic strongyloidiasis)

13. Pelvic surgery (eg, hysterectomy)

14. Porphyria, acute intermittent

15. Retractile mesenteritis

16. Urinary retention

17. Vitamin D deficiency

\section{References}

1. Eisenberg RL: Gastrointestinal Radiology: A Pattern Approach. (ed 3) Philadelphia: Lippincott, 1996, pp 447-452 
2. Gilchrist AM, Mills JOM, Russell CFJ: Acute large bowel pseudo-obstruction. Clin Radiol 1985;36:401-404

3. Spechler SJ, Nath BJ: Case records of the Massachusetts General Hospital, Case 43. N Engl J Med 1985;313: 1070-1079

\section{Gamut G-80}

\section{SENTINEL LOOP (LOCALIZED DILATATION OF SMALL AND/OR LARGE BOWEL)}

\section{COMMON}

1. Acute appendicitis (right lower quadrant)

2. Acute cholecystitis (right upper quadrant)

3. Acute diverticulitis (left lower quadrant)

4. Acute pancreatitis (upper or mid-abdomen)

5. Acute ureteral colic (stone)

6. Infarction or ischemia of bowel

7. "Paralytic ileus" (See G-61)

8. Perforated peptic ulcer (upper abdomen)

9. [Small bowel obstruction, early or incomplete]

\section{UNCOMMON}

1. Abdominal trauma

2. Drug effect

3. Gastroenteritis

4. [Normal variant]

5. [Volvulus]

[ ] This condition does not actually cause the gamuted imaging finding, but can produce imaging changes that simulate it.

\section{References}

1. Baker SR, Cho KC: The Abdominal Plain Film with Correlative Imaging. Norwalk, CT: Appleton \& Lange, 1998

2. Eisenberg RL: Gastrointestinal Radiology: A Pattern Approach. (ed 3) Philadelphia: Lippincott, 1996, p 447

\section{Gamut G-81}

\section{APPENDICEAL LESION OR MASS ADJACENT TO APPENDIX}

\section{COMMON}

1. Appendiceal abscess

2. Appendiceal fecalith (calculus)

3. Appendicitis, acute or resolving

4. Carcinoid

5. Ileocecal valve, normal or prolapsed

6. Postoperative (eg, inverted stump; surgical deformity; adhesions)

\section{UNCOMMON}

1. [Appendix hernia]

2. Crohn's disease

3. [Diverticulum]

4. Endometrial implant

5. Extrinsic mass

6. Foreign body

7. Invagination or intussusception of appendix

8. Mucocele

9. Myxoglobulosis

10. Neoplasm, benign (eg, gastrointestinal stromal tumor $_{\mathrm{g}}$; lipoma; polyp $_{\mathrm{g}}$ )

11. Neoplasm, malignant, primary (eg, carcinoma; lymphoma ${ }_{\mathrm{g}}$ ) or metastatic implant or extension

12. Parasitic disease (eg, amebiasis; trichuriasis; ascariasis; anisakiasis; schistosomiasis; angiostrongyliasis costaricensis)

13. Tuberculosis

14. Typhoid fever

15. Ulcerative colitis

16. Yersinia enterocolitis

[ ] This condition does not actually cause the gamuted imaging finding, but can produce imaging changes that simulate it.

\section{References}

1. Balthazar EJ: Diseases of the appendix. In: Gore RM, Levine MS: Textbook of Gastrointestinal Radiology. (ed 2) Philadelphia: WB Saunders, 2000, pp 1123-1150 
2. Govoni AF: Radiology of para and pericecal lesions. Rev Interamer Radiol 1984; 9:181-185

3. Jeffrey RB, Jr. Chapter 19. Gastrointestinal tract and peritoneal cavity. In: McGahan JP, Goldberg BB (eds): Diagnostic Ultrasound. Philadelphia: Lippincott-Raven, 1997

4. Wolverson MK, Jagannadharao B, Sundaram M, et al: CT as a primary diagnostic method in evaluating intraabdominal abscess. AJR 1979;133:1089-1095

\section{Gamut G-82-S}

\section{CONGENITAL ANOMALIES AND VARIATIONS OF THE APPENDIX}

\section{COMMON}

1. Abnormal length (under $2 \mathrm{~cm}$ or over $25 \mathrm{~cm}$ )

2. Abnormal location (eg, retrocecal appendix; malposition or malrotation of cecum)

3. Abnormal origin (eg, close to or far from ileocecal valve; high on cecum)

\section{UNCOMMON}

1. Absence of appendix

2. Double appendix

3. Primitive appendix (very thin lumen)

\section{References}

1. Balthazar EJ, Gade M: The normal and abnormal development of the appendix. Radiology 1976;121:599-604.

2. Balthazar EJ: The appendix. In: Gore RM, Levine MS: Textbook of Gastrointestinal Radiology. (ed 2) Philadelphia: WB Saunders, 2000, pp 1123-1150

\section{Gamut G-83}

\section{SOLITARY FILLING DEFECT IN THE COLON}

\section{COMMON}

1. Carcinoma

2. Diverticulitis; pericolic abscess (See G-89)

3. Fecal mass or impaction

4. Intussusception; ileal prolapse

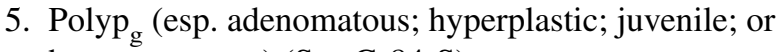
hamartomatous) (See G-84-S)

\section{UNCOMMON}

1. Amyloidoma

2. Carcinoid

3. Crohn's disease

4. Cyst (duplication or other)

5. Endometrioma

6. Extramedullary plasmacytoma

7. Foreign body; gallstone; food particle; bezoar

8. Intramural hematoma

9. Inverted colonic diverticulum

10. Lymphoma

11. Metastasis (incl. invasive neoplasm)

12. Mucormycoma

13. Neoplasm, benign (eg, lipoma; hemangioma; leiomyoma or other gastrointestinal stromal tumor $_{\mathrm{g}}$ )

14. Parasitic disease (esp. ameboma; schistosomal granuloma; helminthoma; anisakiasis, Ascaris bolus)

15. Periappendiceal abscess

16. Postoperative (eg, anastomosis; suture granuloma)

17. Pseudopolyp, "giant" type (in ulcerative colitis)

18. Pseudotumor (eg, fibrous band; adhesion)

19. Sarcoma (incl. Kaposi)

20. Solitary rectal ulcer syndrome

21. Tuberculoma

22. Varix; hemorrhoid

23. Villous adenoma

\section{References}

1. Eisenberg RL: Gastrointestinal Radiology: A Pattern Approach. (ed 3) Philadelphia: Lippincott, 1996, pp 683-717 
2. Gelfand DW: Gastrointestinal Radiology. New York: Churchill Livingstone, 1984, p 325

3. Rubesin SE, Saul SH, Laufer I: Carpet lesions of the colon. Radiographics 1985;5:537-552

\section{Gamut G-84-S}

CLASSIFICATION OF COLONIC TUMORS AND TUMOR-LIKE LESIONS

\section{EPITHELIAL TUMORS}

\section{BENIGN-ADENOMA}

1. Tubular

2. Villous

3. Tubulovillous

\section{MALIGNANT}

1. Adenocarcinoma

2. Adenoacanthoma

3. Adenosquamous carcinoma

4. Squamous cell carcinoma

5. Basaloid (cloacogenic) carcinoma

6. Carcinosarcoma

\section{NEUROENDOCRINE TUMORS}

1. Adenocarcinoid tumor

2. Carcinoid tumor

\section{NONEPITHELIAL TUMORS}

\section{BENIGN}

1. Smooth-muscle tumor

2. Neurilemmoma

3. Lipoma

4. Vascular tumor

\section{HEMATOPOIETIC AND LYMPHOID TUMORS}

\section{UNCLASSIFIED TUMORS}

\section{SECONDARY (METASTATIC) TUMORS}

VII. NONNEOPLASTIC (TUMOR-LIKE)

\section{LESIONS}

\section{HAMARTOMA}

1. Peutz-Jeghers polyp

2. Juvenile polyp

\section{HETEROTOPIA}

1. Colitis cystica profunda

2. Hyperplastic polyp

3. Lymphoid polyp

4. Inflammatory polyp

\section{References}

1. Fengolio-Preiser CM: Gastrointestinal Pathology (ed 2) Philadelphia: Lippincott-Raven, 1999

2. Olmsted WW, Ros PR, Sobin LH, et al: The solitary colonic polyp: Radiologic-histologic differentiation and significance. Radiology 1986;160:9-16

\section{Gamut G-85}

\section{ANNULAR ("APPLE CORE" OR “NAPKIN RING”) LESION OF THE COLON}

\section{COMMON}

1. Carcinoma

\section{UNCOMMON}

1. Ameboma

2. [Circular muscle contraction (colonic "sphincter")]

3. Diverticulitis (esp. with submucosal abscess)

4. Helminthoma

5. Lymphoma

6. Stricture, chronic localized (eg, Crohn's disease; ulcerative colitis; ischemic colitis; lymphogranuloma venereum)

7. Tuberculoma

8. Villous adenoma

[ ] This condition does not actually cause the gamuted imaging finding, but can produce imaging changes that simulate it. 


\section{References}

1. Eisenberg RL: Gastrointestinal Radiology: A Pattern Approach (ed 3). Philadelphia: Lippincott, 1996, pp 640-682

2. Gore RM: Colon: Differential diagnosis. In: Gore RM, Levine MS: Textbook of Gastrointestinal Radiology. (ed 2) Philadelphia: WB Saunders, 2000, pp 1159-1165

\section{Gamut G-86}

\section{SEGMENTAL NARROWING OF THE COLON}

\section{COMMON}

1. Abscess or other extrinsic inflammatory process (eg, pericolic) (See G-89)

2. Carcinoma (esp. "apple-core" adenocarcinoma or scirrhous)

3. [Circular muscle contraction or spasm, usually transient]

4. Crohn's disease

5. Diverticulitis

6. Endometriosis

7. Ischemic colitis (See G-69)

8. Postoperative deformity (eg, adhesion; narrow anastomosis)

9. Ulcerative colitis

\section{UNCOMMON}

1. Actinomycosis

2. Adhesive bands

3. Amyloidosis

4. Carcinoid

5. Carcinoma developing in ulcerative colitis or Crohn's disease or adjacent to ureterosigmoidostomy stoma

6. Cathartic colon; caustic colitis

7. Colitis, other (eg, herpes; cytomegalovirus; fungal)

8. Foreign body perforation with pericolic abscess

9. Intramural hematoma

10. Lymphogranuloma venereum

11. Lymphoma ${ }_{\mathrm{g}}$
12. Metastatic disease (eg, hematogenous or lymphangitic spread; peritoneal seeding; or direct extension to colon)

13. Neoplasm of colon, benign (eg, lipoma, gastrointestinal stromal tumor $\mathrm{r}_{\mathrm{g}}$ )

14. Neoplasm, extrinsic (eg, pancreatic; ovarian; renal)

15. Pancreatitis with direct spread to transverse colon or splenic flexure

16. Parasitic disease (eg, amebiasis/ameboma; schistosomiasis; helminthoma; strongyloidiasis; angiostrongyliasis costaricensis)

17. Pelvic lipomatosis

18. Radiation fibrosis

19. Retractile mesenteritis

20. Sarcoma (incl. Kaposi)

21. Stricture, idiopathic

22. Tuberculosis

23. Typhlitis

24. Ulcer of colon (esp. solitary rectal ulcer syndrome)

[ ] This condition does not actually cause the gamuted imaging finding, but can produce imaging changes that simulate it.

\section{References}

1. Eisenberg RL: Gastrointestinal Radiology: A Pattern Approach. (ed 3) Philadelphia: Lippincott, 1996, pp 640-682

2. Gore RM: Colon: Differential diagnosis. In: Gore RM, Levine MS: Textbook of Gastrointestinal Radiology. (ed 2) Philadelphia: WB Saunders, 2000, pp 1159-1165

3. McDonald JB, Middleton PJ: Tuberculosis of the colon simulating carcinoma. Radiology 1976;118:293-294

4. Simpkins KC, Young AC: The differential diagnosis of large bowel strictures. Clin Radiol 1971;22:449-457

5. Twersky J, Himmelfarb E: Right colonic adhesions. Radiology 1976;120:37-40

\section{Gamut G-87}

\section{MULTIPLE FILLING DEFECTS IN THE COLON}

\section{COMMON}

1. Artifacts (eg, feces; air bubbles; corn or other food particles; mucus strands or globules; oil droplets; foreign bodies)

2. [Diverticulosis] 
3. Normal lymphoid follicles

4. Polyposis, familial

5. Polyps $_{\mathrm{g}}$ (See G-84-S)

6. Pseudopolyps (esp. in ulcerative colitis)

\section{UNCOMMON}

1. Amebomas

2. Amyloidosis

3. Bannayan-Riley-Ruvalcaba S.

4. Carcinomas, multiple

5. Colitis cystica profunda

6. Colitis, other types (eg, pseudomembranous; ischemic; Yersinia; cytomegalovirus; Behçet S.)

7. Cowden S. (multiple hamartoma S.)

8. Cronkhite-Canada S.

9. Cystic fibrosis (mucoviscidosis)

10. Endometriosis

11. Gardner S.

12. Hemangiomas

13. Juvenile polyposis

14. Lipomatous polyposis

15. Lymphoma ${ }_{\mathrm{g}}$; leukemic infiltration

16. Malakoplakia

17. Metastases

18. Neurofibromatosis

19. Nodular lymphoid hyperplasia (if over $4 \mathrm{~mm}$ in size, may be due to AIDS with lymphoid hyperplasia); lymphoid polyps

20. Parasites, intraluminal (eg, Ascaris; Trichuris)

21. Peutz-Jeghers S. (alimentary tract polyposis)

22. Pneumatosis cystoides intestinalis

23. Polyposis syndromes, other (See G-106)

24. Schistosomiasis (inflammatory polyps)

25. Turcot $\mathrm{S}$.

26. Urticaria, incl. hives, blebs, submucosal edema (secondary to chronic ischemia; colon obstruction; herpes zoster or Yersinia colitis; Chagas' disease)

27. Varices; hemorrhoids

[ ] This condition does not actually cause the gamuted imaging finding, but can produce imaging changes that simulate it.

\section{References}

1. Danoff DM, Nisenbarum BL, Stewart WB, et al: Segmental polypoid lipomatosis of the colon. AJR 1977;128:858-860
2. Eisenberg RL: Gastrointestinal Radiology: A Pattern Approach. (ed 3) Philadelphia: Lippincott, 1996, pp 718-747

3. Gelfand DW: Gastrointestinal Radiology. New York: Churchill Livingstone, 1984, p 329

4. Taybi H, Lachman RS: Radiology of Syndromes, Metabolic Disorders, and Skeletal Dysplasias. (ed 4) St. Louis: MosbyYear Book, 1996, p 964

5. Teplick JG, Haskin ME: Roentgenologic Diagnosis. (ed 3) Philadelphia: WB Saunders, 1976

6. Wolfson JJ, Goldstein G, Krivit W, et al: Lymphoid hyperplasia of the large intestine associated with dysgammaglobulinemia: Report of a case. AJR 1970;108:610-614

7. Yousefzadeh DK: Urticaria of the colon. Radiology 1979; 132:315-316

\section{Gamut G-88}

\section{COLITIS (ULCERATION, EDEMA, SPASM); ALSO THICKENING OF THE COLON WALL ON CT, US, OR MRI}

\section{COMMON}

1. Amebiasis

2. Carcinoma, esp. scirrhous or mucinous adenocarcinoma

3. Crohn's disease

4. Diverticulitis

5. Helicobacter fetus colitis

6. Ischemic colitis (See G-69)

7. Ulcerative colitis

\section{UNCOMMON}

1. Actinomycosis

2. Amyloidosis

3. Bacillary dysentery (shigellosis)

4. Behçet S.

5. Bowel edema from cirrhosis, protein loss, portal hypertension

6. Cathartic colon; enema abuse (caustic colitis)

7. Chronic granulomatous disease of childhood

8. Colitis cystica profunda

9. Collagenous colitis 
10. Drug therapy (esp. steroids; NSAIDs; antibiotics; chemotherapeutic agents; cimetidine; anti-fungal flucytosine; gold; methyldopa)

11. Fungus disease (eg, histoplasmosis; mucormycosis; candidiasis)

12. Graft-versus-host disease

13. Lymphogranuloma venereum (chlamydia infection)

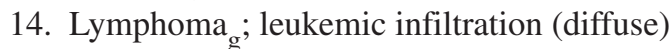

15. Mercury poisoning

16. Metastatic disease

17. Neoplasm, other (eg, gastrointestinal stromal tumor $_{\mathrm{g}}$; Kaposi sarcoma)

18. Nonspecific benign ulceration of colon

19. Pancreatitis with involvement of transverse colon or splenic flexure

20. Parasitic disease, other (esp. schistosomiasis; strongyloidiasis; helminthoma; Chagas' disease; anisakiasis and angiostrongyliasis costaricensisileocecal region)

21. Postoperative colitis (incl. diversion colitis in isolated segment after proximal colostomy or ileostomy; postrectal biopsy ulceration)

22. Proctitis (nonspecific; chemical-paraldehyde; in male homosexuals, often due to gonorrhea, lymphogranuloma venereum, or herpes simplex)

23. Pseudomembranous or necrotizing colitis (eg, Clostridium difficile infection, esp. after antibiotic therapy; postoperative; or proximal to colon obstruction)

24. Radiation colitis

25. Salmonella colitis

26. Solitary rectal ulcer $\mathrm{S}$.

27. Staphylococcal colitis (eg, after oral tetracycline therapy)

28. Stercoral colitis (in obstruction)

29. Tuberculosis

30. Typhlitis

31. Uremic colitis; hemolytic-uremic S.

32. Urticaria ("colon hives" secondary to chronic ischemia; colon obstruction; herpes zoster or Yersinia colitis; Chagas' disease)

33. Viral infection (eg, herpes simplex-anorectal herpes; herpes zoster; cytomegalovirus-esp. in AIDS; rotavirus)

34. Yersinia colitis

\section{References}

1. Eisenberg RL: Gastrointestinal Radiology: A Pattern Approach. (ed 3) Philadelphia: Lippincott, 1996, pp 601-639

2. Gelfand DW: Gastrointestinal Radiology. New York: Churchill Livingstone, 1984, p 315

3. Gore RM: Inflammatory disease. In: Margulis AR: Modern Imaging of the Alimentary Tube. New York: Springer-Verlag, 1998, pp 185-216

4. Gore RM: Colon: Differential diagnosis. In: Gore RM, Levine MS: Textbook of Gastrointestinal Radiology. (ed 2) Philadelphia: WB Saunders, 2000, pp 1159-1165

5. Halpert RD, Feczko PJ: Gastrointestinal Radiology; The Requisites. (ed 2) St. Louis: Mosby-Year Book, 1999

6. Marshak RH, Lindner AE, Maklansky D: Radiology of the Colon. Philadelphia: WB Saunders, 1980

7. Miller VE, Han SY, Witten DM: Reticular mosaic (urticarial) pattern of the colonic mucosa in Yersinia colitis. Radiology 1983;146:307-308

8. Palmer PES, Reeder MM: The Imaging of Tropical Diseases. (ed 2) Heidelberg: Springer-Verlag, 2001

9. Teplick JG, Haskin ME: Roentgenologic Diagnosis. (ed 3) Philadelphia: WB Saunders, 1976

\section{Gamut G-89 \\ PERICOLIC ABSCESS}

\section{COMMON}

1. Appendicitis

2. Crohn's disease

3. Diverticulitis

4. Neoplasm of colon, perforated primary (esp. carcinoma) or metastatic

5. Pancreatitis

6. Trauma, external or iatrogenic (eg, enema)

7. Tubo-ovarian abscess

\section{UNCOMMON}

1. Actinomycosis

2. Foreign body perforation

3. Idiopathic

4. Ischemic colitis 
5. Lymphogranuloma venereum (Chlamydia infection)

6. Parasitic disease (esp. amebiasis; schistosomiasis; helminthoma; anisakiasis or angiostrongyliasis costaricensis-ileocecal region)

7. Postoperative (eg, suture leak; talc granuloma)

8. Renal infection or abscess

9. Tuberculosis

10. Ulcerative colitis

\section{References}

1. Ryan JM, Mueller PR: Abdominal abscess. In: Gore RM, Levine MS: Textbook of Gastrointestinal Radiology. (ed 2) Philadelphia: WB Saunders, 2000, pp 1234-1249

2. Wolverson MK, Jagannadharao B, Sundaram M, et al: CT as a primary diagnostic method in evaluating intraabdominal abscess. AJR 1979;133:1089-1095

\section{Gamut G-90}

\section{DOUBLE TRACKING OF BARIUM IN THE DISTAL COLON*}

\section{COMMON}

1. Crohn's disease

2. Diverticulitis

\section{UNCOMMON}

1. Carcinoma of colon

* Barium in an extraluminal sinus tract paralleling the bowel lumen.

\section{Reference}

1. Eisenberg RL: Gastrointestinal Radiology: A Pattern Approach. (ed 3) Philadelphia: Lippincott, 1996, pp 784-787

\section{Gamut G-91}

\section{SMOOTH COLON}

\section{COMMON}

1. Cathartic or enema abuse

2. Chronic obstruction (eg, megacolon; Chagas' disease)

3. Ischemic colitis, late (See G-69)

4. Ulcerative colitis, chronic

\section{UNCOMMON}

1. Amyloidosis

2. Bacillary dysentery, chronic (shigellosis)

3. Carcinoma, scirrhous

4. Lymphogranuloma venereum, late (Chlamydia infection)

5. Lymphoma

6. Parasitic disease, late (eg, schistosomiasis; strongyloidiasis; amebic stricture)

7. Radiation colitis, late

\section{References}

1. Caroline DF, Evers K: Colitis: radiographic features and differentiation of idiopathic inflammatory bowel disease. Radiol Clin North Am 1987;25:47-63

2. Kim SK, Gerle RD, Rozanski R: Cathartic colitis. AJR 1978;130:825-829

\section{Gamut G-92}

\section{COLONIC DISTENTION WITHOUT OBSTRUCTION}

\section{COMMON}

1. Acute nonobstructive distention, "paralytic ileus" (eg, postoperative; peritonitis; appendicitis; pancreatitis; typhoid fever) (See G-61)

*2. Chronic constipation; cathartic abuse

3. Electrolyte imbalance (hypokalemia; hypochloremia; calcium abnormality) 
*4. Functional megacolon (psychogenic; idiopathic)

*5. Hirschsprung's disease

6. Mesenteric infarction (See G-69)

7. Shock; septicemia; hypoxia

8. Toxic megacolon (See G-94)

9. Trauma (esp. spine or lower rib injury; intramural hematoma)

10. Ureteral colic

\section{UNCOMMON}

*1. Aerophagia

*2. Amyloidosis; familial Mediterranean fever (familial recurrent polyserositis)

*3. Ceroidosis (vitamin E depletion)

*4. Chagas' disease

*5. Connective tissue disease (collagen disease) (esp. scleroderma; polyarteritis nodosa)

*6. Cystic fibrosis (mucoviscidosis)

*7. Drug therapy (eg, Pro-Banthine; hexamethonium; chlorpromazin; benztropine; morphine; L-dopa; atropine)

*8. Endocrine disturbance (eg, adrenal insufficiency; multiple endocrine neoplasia (MEN) S.; hypothyroidism; hypoparathyroidism)

9. Functional (eg, diabetic coma; lead colic; sickle cell crisis; tabes; porphyria; pheochromocytoma)

10. Idiopathic intestinal pseudo-obstruction

11. Kawasaki disease

*12. Muscular, neurologic, or psychiatric disorder ${ }_{g}$ (eg, amyotonia congenita \{Oppenheim's disease\}; multiple sclerosis; parkinsonism, Riley-Day S. \{familial dysautonomia\}; senility; schizophrenia; brain damage; paralysis)

13. Neurofibromatosis (esp. plexiform)

14. Pneumonia or other acute thoracic disease (eg, myocardial infarction; heart failure)

*15. Renal failure; uremia; urinary retention

16. Retroperitoneal hemorrhage

17. Spinal cord lesion; paraplegia

*18. Sprue

* Chronic.

\section{References}

1. Baker DA, Morin ME, Tan A, et al: Colonic ileus. Indication for prompt decompression. JAMA 1979; 241:2633-2634

2. Baker SR, Cho KC: The Abdominal Plain Film with Correlative Imaging. Norwalk, CT: Appleton \& Lange, 1998

3. Bode WE, Beart RW, Spencer RJ, et al: Colonoscopic decompression for acute pseudobstruction of the colon (Ogilvie's syndrome): Report of 22 cases and review of the literature. Am J Surg 1984;147:243-254

4. Gilchrist AM, Mills JOM, Russell CFJ: Acute large-bowel pseudo-obstruction. Clin Radiol 1985;36:401-404

5. Meyers MA: Colonic ileus. Gastrointest Radiol 1977;2: 37-40

6. Passail G, Benacerraf R: Acute pseudobstruction of the colon. Ann Radiol 1979; 22:508-514

7. Vanek VW, Al-Salti M: Acute pseudobstruction of the colon (Ogilvie's syndrome): An analysis of 400 cases. Dis Colon Rectum 1986; 29:203-210

\section{Gamut G-93 MEGACOLON}

\section{COMMON}

1. Distal obstructing lesion (esp. carcinoma)

*2. Functional (psychogenic; idiopathic)

*3. Hirschsprung disease

*4. Imperforate anus; colon or rectal atresia; anal stenosis

*5. Nonobstructive distention ("paralytic ileus")

6. Scleroderma; dermatomyositis

*7. Toxic megacolon (See G-94)

\section{UNCOMMON}

*1. Aerophagia

2. Amyloidosis

3. Chagas' disease

*4. Chronic idiopathic intestinal pseudo-obstruction

*5. Cystic fibrosis (mucoviscidosis); meconium plug S. (dilated colon proximal to plug)

6. Diabetes

*7. Drug therapy (esp. Pro-Banthine; phenothiazine; anti-Parkinsonism agents) 
*8. Duplication

*9. Fetal cytomegalovirus infection

*10. Hypothyroidism (esp. myxedema; cretinism)

*11. Immobilization, prolonged (eg, cast S.)

*12. Multiple endocrine neoplasia (MEN) S. (See J-5)

*13. Muscular disorder ${ }_{\mathrm{g}}$ (eg, muscular dystrophy)

*14. Neurologic disorder ${ }_{\mathrm{g}}$ (eg, plexiform neurofibromatosis; meningomyelocele; spina bifida; paralysis; neuronal intestinal dysplasia; Parkinsonism, brain damage)

*15. Obstruction of colon from chronic stricture (eg, lymphogranuloma venereum or other rectal stricture; Crohn's disease; postoperative scarring*; post-necrotizing enterocolitis*)

*16. Purgative abuse

*17. Riley-Day S. (familial dysautonomia)

*18. Sacrococcygeal teratoma; other pelvic neoplasm

*19. Small left colon S. (dilated colon proximally)

*20. Sotos S. (cerebral gigantism)

*21. Steatorrhea; celiac disease; sprue

* Megacolon in an infant or child.

\section{References}

1. Gelfand DW: Gastrointestinal Radiology. New York: Churchill Livingstone, 1984, p 304

2. Messmer JM: Gas and soft tissue abnormalities. In: Gore RM, Levine MS: Textbook of Gastrointestinal Radiology. (ed 2) Philadelphia: WB Saunders, 2000, pp 157-177

3. Solano Jr FX, Starling RC, Levey GS: Myxedema megacolon. Arch Intern Med 1985;145:231

4. Taybi H, Lachman RS: Radiology of Syndromes, Metabolic Disorders, and Skeletal Dysplasias. (ed 4) St. Louis: MosbyYear Book, 1996, p 964

\section{Gamut G-94
TOXIC MEGACOLON}

\section{COMMON}

1. Ulcerative colitis

\section{UNCOMMON}

1. Amebic colitis
2. Bacillary dysentery, acute (shigellosis)

3. Behçet $\mathrm{S}$.

4. Cholera

5. Crohn's disease

6. Enterocolitis, other (necrotizing; pseudomembranous; hemorrhagic)

7. Helicobacter fetus colitis

8. Hirschsprung disease

9. Ischemic colitis

10. Strongyloidiasis

11. Typhoid fever

\section{References}

1. Eisenberg RL: Gastrointestinal Radiology: A Pattern Approach. (ed 3) Philadelphia: Lippincott, 1996, pp 768-772

2. Gelfand DW: Gastrointestinal Radiology. New York: Churchill Livingstone, 1984, p 308

\section{Gamut G-95-1}

\section{COLON OF REDUCED CALIBER (MICROCOLON) IN A NEWBORN}

1. "Apple peel" intestinal atresia $S$.

2. Berdon S. (megacystis-microcolon-intestinal hypoperistalsis $\mathrm{S}$.)

3. Colon atresia (microcolon to point of atresia)

4. Congenital ileal stenosis, web

5. Distal jejunal atresia (occas. total microcolon of disuse)

6. Hirschsprung disease (aganglionic distal segment may be small)

7. Ileal atresia (total microcolon of disuse)

8. Meconium ileus, esp. with cystic fibrosis \{mucoviscidosis\} (total microcolon of disuse)

9. Meconium plug S. (entire colon may be slightly small)

10. Microcolon of prematurity

11. Small left colon S. (rectum normal, colon small to splenic flexure)

12. Total aganglionosis coli (entire colon may be slightly small—question mark colon) 


\section{Reference}

1. Taybi H, Lachman RS: Radiology of Syndromes, Metabolic Disorders, and Skeletal Dysplasias. (ed 4) St. Louis: MosbyYear Book, 1996, p 964

\section{Gamut G-95-2}

\section{MECONIUM PLUG IN A NEWBORN}

1. Cystic fibrosis (mucoviscidosis) with meconium ileus

2. Hirschsprung disease

3. Meconium plug $S$.

4. Small left colon $S$.

\section{References}

1. Fernbach SK: Neonatal gastrointestinal radiology. In: Gore RM, Levine MS: Textbook of Gastrointestinal Radiology. (ed 2) Philadelphia: WB Saunders, 2000, pp 2042-2073

2. Taybi H, Lachman RS: Radiology of Syndromes, Metabolic Disorders, and Skeletal Dysplasias. (ed 4) St. Louis: MosbyYear Book, 1996, p 964

\section{Gamut G-96 \\ CECAL LESION}

\section{COMMON}

1. Amebiasis

2. Appendiceal stump or lesion (See G-81)

3. Carcinoma

4. Crohn's disease

5. [Fecal matter; pill; food particle; foreign body]

6. Ileocecal valve, normal or fatty ("lipoma"); ileocecal prolapse

7. Ileocecal intussusception (eg, idiopathic; lymphoma; Meckel's diverticulum; appendix)

8. Polyp

9. Ulcerative colitis

10. Volvulus

\section{UNCOMMON}

1. Actinomycosis

2. Benign neoplasm (esp. villous adenoma; angioma; gastrointestinal stromal tumor ${ }_{\mathrm{g}}$; lipoma)

3. Carcinoid

4. Cathartic abuse

5. Cecal diaphragm, web, or adhesion

6. Cytomegalovirus infection (esp. in AIDS)

7. Diverticulosis; diverticulitis

8. Duplication

9. Endometriosis

10. Enterolith

11. Foreign body perforation

12. [Gallstone]

13. Ileus of cecum

14. Lymphoma (incl. Burkitt lymphoma)

15. Metastasis (esp. colon, stomach, pancreas, ovary)

16. Parasitic disease, other (eg, schistosomiasis; strongyloidiasis; ascariasis; trichuriasis; anisakiasis; helminthoma; angiostrongyliasis costaricensis)

17. Pneumatosis cystoides intestinalis

18. Tuberculosis

19. Typhlitis

20. Typhoid fever

21. Ulcer, solitary

22. Yersinia enterocolitis

[ ] This condition does not actually cause the gamuted imaging finding, but can produce imaging changes that simulate it.

\section{References}

1. Eisenberg RL: Gastrointestinal Radiology: A Pattern Approach. (ed 3) Philadelphia: Lippincott, 1996, pp 555-598

2. Felson B, Wiot JF: Case of the Day. Springfield, IL: CC Thomas, 1967, p3

3. Felson B, Wiot JF: Some interesting right lower quadrant entities: Myxoglobulosis of the appendix, ileal prolapse, diverticulitis, lymphoma, endometriosis. Radiol Clin North Am 1969;7:83-95

4. Govoni AF: Radiology of para and pericecal lesions. Rev Interamer Radiol 1984;9:181-185

5. Margolies MN, Welch CE: Case records of the Massachusetts General Hospital. Case 38-1976. N Engl J Med 1976; 295:666-670

6. Szucs RA, Wolf EL, Gramm HF, et al: Miscellaneous abnormalities of the colon. In: Gore RM, Levine MS: Textbook of Gastrointestinal Radiology. (ed 2) Philadelphia: WB Saunders, 2000, pp 1089-1122 


\section{Gamut G-97}

\section{CONICAL OR CONTRACTED CECUM}

\section{COMMON}

1. Amebiasis

2. Appendicitis; appendiceal abscess

3. Crohn's disease

\section{UNCOMMON}

1. Actinomycosis; South American blastomycosis

2. Anisakiasis; angiostrongyliasis costaricensis

3. Carcinoma

4. Cathartic abuse

5. Cytomegalovirus colitis (esp. in AIDS)

6. Diverticulitis of cecum (esp. following perforation)

7. Idiopathic

8. Lymphoma ${ }_{\mathrm{g}}$; leukemia (esp. in children after treatment)

9. Metastatic disease (eg, from carcinoma of stomach, colon, pancreas, ovary)

10. Radiation therapy

11. Tuberculosis (Stierlin sign)

12. Typhlitis

13. Typhoid fever

14. Ulcerative colitis

15. Yersinia enterocolitis

\section{References}

1. Eisenberg RL: Gastrointestinal Radiology: A Pattern Approach. (ed 3) Philadelphia: Lippincott, 1996, pp 589-598

2. Gore RM: Colon: Differential diagnosis. In: Gore RM, Levine MS: Textbook of Gastrointestinal Radiology. (ed 2) Philadelphia: WB Saunders, 2000, pp 1159-1165

3. Kreel L: Outline of Radiology. New York: AppletonCentury-Crofts, 1971, p 158

\section{Gamut G-98}

\section{ENLARGEMENT OF THE ILEOCECAL VALVE}

\section{COMMON}

1. Amebiasis

2. Crohn's disease

3. Fatty infiltration (lipomatosis)

4. Intussusception

5. Neoplasm, benign or malignant (esp. villous adenoma; polyp $\mathrm{g}_{\mathrm{g}}$; lipoma; carcinoid; adenocarcinoma; lymphoma $_{\mathrm{g}}$ )

6. Normal variant ("hypertrophy")

\section{UNCOMMON}

1. Actinomycosis

2. Anisakiasis

3. Cathartic abuse

4. Foreign body, impacted

5. Gallstone, impacted

6. Ileocolic prolapse

7. Intramural hematoma

8. Lymphoid hyperplasia

9. Tuberculosis

10. Typhoid fever

11. Ulcerative colitis

12. Yersinia enterocolitis

\section{Reference}

1. Eisenberg RL: Gastrointestinal Radiology: A Pattern Approach. (ed 3) Philadelphia: Lippincott, 1996, pp 555-565 


\section{Gamut G-99}

\section{ANTERIOR INDENTATION ON THE RECTOSIGMOID JUNCTION}

\section{COMMON}

1. Abscess, pelvic (eg, bacterial; PID; amebiasis; schistosomiasis; LGV)

2. Ascites (in erect position)

3. Carcinoma of ovary, cervix, uterus, bladder, or rectosigmoid colon

4. Endometriosis

5. Extrinsic pelvic neoplasm, other (eg, teratoma; dermoid or other ovarian cyst; uterine fibroid)

6. Metastasis, peritoneal (Blumer shelf) (esp. from carcinoma of colon, stomach, pancreas, ovary)

\section{UNCOMMON}

1. Aneurysm of internal iliac artery

2. Hematoma

3. Hydatid cyst

4. Lymphocele

5. Lymphoma $\mathrm{g}_{\mathrm{g}}$, other lymphadenopathy

6. Pelvic lipomatosis

7. Postsurgical sling repair for rectal prolapse

8. Rectovaginal fistula (eg, LGV) (See G-101)

9. Retroperitoneal fibrosis

10. Urinoma

11. Vaginal lesion (eg, carcinoma; hematocolpos)

\section{Reference}

1. Schulman A, Fataar S: Extrinsic stretching, narrowing, and anterior indentation of the rectosigmoid junction. Clin Radiol 1979;30:463-469

\section{Gamut G-100}

\section{RECTAL DISEASE ON BARIUM ENEMA}

\section{COMMON}

1. Abscess

2. Carcinoma (adenocarcinoma; scirrhous; cloacogenic; anal)

3. Congenital anomaly (eg, Hirschsprung disease; imperforate anus)

4. Crohn's disease

5. Endometriosis

6. Metastasis; invasion from adjacent neoplasm

7. Polyp

8. Prolapse

9. Radiation proctitis

10. Trauma (sexual; iatrogenic; puerperal or other)

11. Ulcerative colitis

12. Villous adenoma

\section{UNCOMMON}

1. Actinomycosis

2. Amyloidosis

3. Bacillary dysentery (shigellosis)

4. Carcinoid

5. Colitis cystica profunda

6. Diverticulitis

7. Ischemic colitis

8. Lymphogranuloma venereum (Chlamydia infection)

9. Lymphoma ${ }_{\mathrm{g}}$

10. Neoplasm, benign (eg, gastrointestinal stromal tumor ${ }_{g}$; lipoma; angioma)

11. Opportunistic infection (eg, herpes)

12. Parasitic disease (esp. amebiasis; schistosomiasis; trichuriasis; Chagas' disease)

13. Pelvic lipomatosis

14. Pneumatosis cystoides intestinalis

15. Proctitis (idiopathic; gonorrheal; chemicalparaldehyde)

16. Retroperitoneal fibrosis 
17. Solitary rectal ulcer syndrome

18. Tuberculosis

19. Varices; hemorrhoids

\section{Reference}

1. Hama Y, Okizuka H, Odajima K, et al: Gastrointestinal stromal tumor of the rectum. Eur Radiol 2001;11:216-219

\section{Gamut G-101}

\section{RECTOVAGINAL FISTULA}

\section{COMMON}

1. Crohn's disease

2. Diverticulitis

3. Lymphogranuloma venereum (Chlamydia infection)

4. Neoplasm, malignant (eg, carcinoma of rectum, cervix, or vagina; other pelvic carcinoma)

5. Radiation therapy

6. Trauma (external; sexual; puerperal; iatrogenic)

\section{UNCOMMON}

1. Abscess (eg, appendiceal; tubovarian; perirectal)

2. Actinomycosis

3. Endometriosis

4. Foreign body perforation

5. Imperforate anus or other cloacal anomaly

6. Metastasis

7. Schistosomiasis

8. Tuberculosis

9. Ulcerative colitis

\section{Reference}

1. Greenall MJ, Levine AW, Nolan DJ: Complications of diverticular disease: a review of the barium enema findings. Gastrointest Radiol 1983;8:353-361

\section{Gamut G-102}

\section{INCREASED RETRORECTAL OR PRESACRAL SPACE (See C-50)}

\section{COMMON}

1. Abscess, presacral or pelvic (eg, perforated colon from diverticulitis or carcinoma; tubovarian or periappendiceal abscess)

2. Carcinoma of rectum (esp. adenocarcinoma; also scirrhous; cloacogenic; anal)

3. Crohn's disease

4. Diverticulitis

5. Extrinsic soft tissue mass (incl. ovarian cyst or neoplasm; dermoid; teratoma; enteric duplication cyst; tail gut cyst; lipoma)

6. Hematoma (eg, sacral fracture)

7. Lymphadenopathy

8. Metastatic or invasive malignant neoplasm (eg, from bladder, prostate, ovary, cervix)

9. Normal variant

10. Postoperative (eg, resection of rectosigmoid)

11. Radiation proctitis or fibrosis

12. Sacral or coccygeal lesion (eg, metastasis; myeloma; chordoma; osteosarcoma; chondrosarcoma; giant cell tumor; neurofibroma; sacrococcygeal teratoma; hydatid cyst)

13. Trauma (external; sexual; puerperal; iatrogenicinstrumentation)

14. Ulcerative colitis

15. Urinoma

\section{UNCOMMON}

1. Amebiasis

2. Amyloidosis of colon

3. Anterior sacral meningocele

4. Colitis cystica profunda; pneumatosis intestinalis

5. Cushing S. (fat deposition)

6. Endometriosis

7. Hemorrhoidal injection

8. Inferior vena cava obstruction (pelvic edema)

9. Inguinal hernia with rectal traction 
10. Ischemic colitis (See G-69)

11. Lymphocele

12. Lymphogranuloma venereum (Chlamydia infection)

13. Lymphoma ${ }_{g}$ of rectum or retrorectal soft tissues

14. Neoplasm, benign, of soft tissues or rectum (incl. hemangioma; hemangioendothelioma; gastrointestinal stromal tumor ${ }_{\mathrm{g}}$; lipoma)

15. Pelvic lipomatosis

16. Pneumoretroperitoneum

17. Proctitis (idiopathic; traumatic; gonorrheal; chemical-paraldehyde)

18. Retroperitoneal fibrosis

19. Sarcoma (eg, rhabdomyosarcoma)

20. Schistosomiasis

21. Tuberculosis

\section{References}

1. Burgener FA, Kormano M: Differential Diagnosis in Conventional Radiology. (ed 2) New York: Thieme Medical Publ, 1991, pp 584-585

2. Eisenberg RL: Clinical Imaging: An Atlas of Differential Diagnosis. (ed 3) Philadelphia: Lippincott-Raven, 1997, pp 420-421

3. Swischuk LE, John SD: Differential Diagnosis in Pediatric Radiology. (ed 2) Baltimore: Williams \& Wilkins, 1995, pp 145-146

4. Teplick SK, Stark P, Clark RE, Metz JR, Shapiro JH: The retrorectal space. Clin Radiol 1978;29:177-184

\section{Gamut G-103}

\section{"THUMBPRINTING" OF THE GASTROINTESTINAL TRACT (MULTIPLE INTRAMURAL DEFECTS)}

\section{COMMON}

1. Crohn's disease

2. Diverticulitis

3. Ischemic colitis with hemorrhage into bowel wall (See G-69)

4. Ulcerative colitis

\section{UNCOMMON}

1. Amyloidosis

2. Angioneurotic edema

3. Carcinoid S.

4. Cytomegalovirus colitis in AIDS

5. Endometriosis

6. Hemolytic-uremic S. (ischemic enterocolitis preceding onset of renal failure in infants)

7. Intramural hematoma or hemorrhage (eg, trauma; hemophilia or other bleeding diathesis; anticoagulant therapy)

8. Lymphoma

9. Metastasis, mural or peritoneal

10. Parasitic disease (esp. amebiasis; strongyloidiasis; schistosomiasis; anisakiasis)

11. Pericolic abscess (See G-89)

12. Pneumatosis cystoides intestinalis

13. Pseudomembranous or necrotizing colitis

14. Retractile mesenteritis, other mesenteric or peritoneal lesion (See G-228 to G-231)

15. Toxic megacolon (See G-94)

16. Typhlitis

17. Urticaria ("colon hives" secondary to chronic ischemia; colon obstruction; herpes zoster or Yersinia colitis; Chagas' disease)

\section{References}

1. Eisenberg RL: Gastrointestinal Radiology: A Pattern Approach. (ed 3) Philadelphia: Lippincott, 1996, pp 773-783

2. Marshak RH, Lindner AE, Maklansky D: Radiology of the Colon. Philadelphia: WB Saunders, 1980

3. Schwartz S, Boley SJ, Schultz L, et al: A survey of vascular diseases of the small intestine. Semin Radiol 1966;1: $178-218$ 


\section{Gamut G-104}

\section{INTRAMURAL HEMATOMA OF THE GASTROINTESTINAL TRACT}

\section{COMMON}

1. Anticoagulant therapy

2. Hemophilia; Christmas disease; other bleeding or clotting disorder ${ }_{\mathrm{g}}$

3. Trauma

\section{UNCOMMON}

1. Connective tissue disease (collagen vascular disease) $)_{\mathrm{g}}$ (esp. polyarteritis nodosa)

2. Drug therapy (eg, cytotoxin)

3. Henoch-Schönlein purpura

4. Idiopathic thrombocytopenic purpura

5. Leukemia

\section{Reference}

1. Felson B, Levin EJ: Intramural hematoma of the duodenum: A diagnostic roentgen sign. Radiology 1954; 63:823-830.

\section{Gamut G-105}

\section{BULL'S-EYE LESION (SOLITARY OR MULTIPLE NODULES IN THE GASTROINTESTINAL TRACT WITH LARGE CENTRAL ULCERATION)}

\section{COMMON}

*1. Gastrointestinal stromal tumor ${ }_{\mathrm{g}}$ (esp. leiomyoma; leiomyosarcoma; neurofibroma)

*2. Lymphoma ${ }_{\mathrm{g}}$

*3. Metastatic melanoma

*4. Peptic ulcer

\section{UNCOMMON}

1. Amyloid tumor

2. Carcinoid
3. Carcinoma

4. Ectopic pancreas

5. Eosinophilic granuloma

*6. Kaposi sarcoma

*7. Metastases (esp. from kidney; pancreas; breast; lung)

* May be multiple.

\section{References}

1. Balthazar EJ, Megibow A, Bryk D, et al: Gastric carcinoid tumors: Radiographic features in eight cases. AJR 1982; 139:1123-1127

2. Cavanagh RC, Buchignani JS Jr, Rulon DB: Metastatic melanoma of the small intestine. Radiology 1971;101: 195-200

3. Eisenberg RL: Gastrointestinal Radiology: A Pattern Approach. (ed 3) Philadelphia: Lippincott, 1996, pp 903-908

4. Marshak RH, Lindner AE: Polypoid lesions of the stomach. Semin Roentgenol 1971; 6:151-167

5. Pandarinath GS, Levine SM, Sorokin JJ, et al: Selective massive amyloidosis of the small intestine mimicking multiple tumors. Radiology 1978;129:609-610

6. Pomerantz H, Margolin HN: Metastases to the gastrointestinal tract from malignant melanoma. AJR 1962;88: 712-717

7. Wolf BS: Observations on roentgen features of benign and malignant gastric ulcers. Semin Roentgenol 1971;6: $140-150$

\section{Gamut G-106}

\section{POLYPOSIS SYNDROMES}

1. Behçet $S$.

2. Blue rubber bleb nevus $S$.

3. Cowden S. (multiple hamartoma S.)

4. Cronkhite-Canada S.

5. Familial adenomatous polyposis (eg, familial polyposis; Gardner S.)

6. Juvenile polyposis of infancy; generalized juvenile polyposis

7. Lipomatous polyposis

8. Peutz-Jeghers S. (alimentary tract polyposis)

9. [Polyps, multiple adenomatous]

(continued) 
10. Ruvalcaba-Myhre-Smith S.

11. Turcot $\mathrm{S}$.

[ ] This condition does not actually cause the gamuted imaging finding, but can produce imaging changes that simulate it.

\section{References}

1. Buck JL, Harned RK: Polyposis syndromes. In: Gore RM, Levine MS: Textbook of Gastrointestinal Radiology. (ed 2) Philadelphia: WB Saunders, 2000, pp 1075-1088

2. Dodds WJ: Clinical and roentgen features of the intestinal polyposis syndromes. Gastrointest Radiol 1976;1:127-142

3. Itzkowitz SH, Kim YS: Colon polyps and polyposis syndromes. In: Feldman M, Scharschmidt BF, Sleisenger MH: Gastrointestinal and Liver Diseases. (ed 6) Philadelphia: WB Saunders, 2000, pp 1865-1905

4. Taybi H, Lachman RS: Radiology of Syndromes, Metabolic Disorders, and Skeletal Dysplasias. (ed 4) St. Louis: MosbyYear Book, 1996, p 964

\section{Gamut G-107}

\section{INTERNAL OR EXTERNAL FISTULA INVOLVING THE GASTROINTESTINAL TRACT}

\section{COMMON}

1. Abscess $_{\mathrm{g}}$ (eg, appendiceal; perirenal)

2. Carcinoma; other malignant neoplasm, primary or metastatic

3. Crohn's disease

4. Diverticulitis

5. Postoperative (eg, surgical complication; dehiscence; ileostomy; colostomy; gastrostomy)

\section{UNCOMMON}

1. Actinomycosis

2. Amebiasis

3. Biliary-enteric fistula (See G-139)

4. Colovesical fistula (esp. diverticulitis; malignancy)

5. Duodenal-renal fistula (eg, tuberculosis; pyelonephritis; duodenal ulcer)

6. Entero-ovarian fistula
7. Foreign body (eg, pin; bone; toothpick)

8. Infarction; ischemic colitis (See G-69)

9. Lymphoma ${ }_{\mathrm{g}}$

10. Peptic ulcer; marginal ulcer

11. Pancreatic fistula (pancreatitis; ruptured pseudocyst; posttraumatic; postsurgical; external drainage of pseudocyst)

12. Prosthetic aortic graft

13. Radiation therapy

14. Rectovaginal, pelvic, or perineal fistula (esp. pelvic inflammatory disease; lymphogranuloma venereum; diverticulitis; Crohn's disease; malignancy) (See G-101)

15. Schistosomiasis

16. Trauma, external or iatrogenic (eg, enema)

17. Tuberculosis

18. Ulcerative colitis

\section{References}

1. Eisenberg RL: Gastrointestinal Radiology: A Pattern Approach (ed 3). Philadelphia: Lippincott, 1996, pp 971-981

2. Gore RM, Calenoff L, Rogers LF: Roentgenographic manifestations of ischemic colitis. JAMA 1979;241:1171-1173

Gamut G-108

\section{NONVISUALIZATION OF THE GALLBLADDER (US, CT, NM)}

\section{COMMON}

1. [Calcified gallbladder wall (porcelain gallbladder); milk of calcium bile]

2. Cholecystitis (acute or chronic; gangrenous; emphysematous)

3. Contracted gallbladder (esp. postprandial)

4. Prior cholecystectomy

5. Technical factors, esp. on ultrasound (eg, gallbladder obscured by gas; obese patient or thin patient with superficial gallbladder) 


\section{UNCOMMON}

1. [Anomalous position of gallbladder (eg, ectopic gallbladder; situs inversus)]

2. Biliary atresia

3. Carcinoma of gallbladder

4. Compression by adjacent mass

5. Congenital absence or hypoplasia

6. Fibrosis of gallbladder

7. Hepatization of the gallbladder (sludge-filled)

8. Hyperalimentation; nonfasting patient

9. Metastatic disease involving gallbladder

10. Pregnancy (last trimester)

11. Syndromes (eg, Dubin-Johnson S.; Kawasaki S.; Mirizzi S.)

[ ] This condition does not actually cause the gamuted imaging finding, but can produce imaging changes that simulate it.

\section{References}

1. Abbitt PL: Ultrasound: A Pattern Approach. New York: McGraw-Hill, 1995

2. Balthazar U, Dunn J, Gonzalez-Diaz S, et al: Agenesis of the gallbladder. South Med J 2000;93:914-915

3. Eisenberg RL: Gastrointestinal Radiology: A Pattern Approach. (ed 3) Philadelphia: Lippincott, 1996, pp 803-808

4. Gore RM: Gallbladder and biliary tract: Differential diagnosis. In: Gore RM, Levine MS: Textbook of Gastrointestinal Radiology. (ed 2) Philadelphia: WB Saunders, 2000, pp 1408-1414

5. Laing FC: Chapter 6. The gallbladder and bile ducts. The liver. In: Rumack CM, Wilson SR, Charboneau JW (eds): Diagnostic Ultrasound. St. Louis: Mosby, 1998, pp 175-223

6. Parulekar SG: Gallbladder and bile ducts. In: McGahan JP, Goldberg BB (eds): Diagnostic Ultrasound. Philadelphia: Lippincott-Raven, 1997, chapter 22

7. Shehadi WH: Radiologic examination of the biliary tract. Radiol Clin North Am 1966;4:463-482

8. Williamson, MR: Abdominal ultrasound. In: Essentials of Ultrasound. Philadelphia: WB Saunders, 1996, p 86

\section{Gamut G-109 \\ SMALL OR CONTRACTED GALLBLADDER (US, CT, MRI)}

\section{COMMON}

1. Acute hepatitis

2. Chronic cholecystitis

3. Postprandial status

\section{UNCOMMON}

1. Adenomyomatosis of gallbladder

2. Congenital hypoplasia or multiseptate gallbladder

3. Cystic fibrosis (mucoviscidosis)

\section{References}

1. Eisenberg RL: Gastrointestinal Radiology: A Pattern Approach. (ed 3) Philadelphia: Lippincott, 1996, pp 809-812.

2. Gore RM: Gallbladder and biliary tract disease: Differential diagnosis. In: Gore RM, Levine MS (eds): Textbook of Gastrointestinal Radiology. ed 2 Philadelphia: WB Saunders, 2000, pp 1408-1415

3. Irie H, Honda H, Kuroiwa T, et al: Pitfalls in MR cholangiopancreatographic interpretation. RadioGraphics 2001; $21: 23-38$

\section{Gamut G-110}

\section{ENLARGED GALLBLADDER (US, CT, MRI)}

\section{COMMON}

1. Acute cholecystitis with cholelithiasis causing obstruction of cystic or common duct

2. Diabetes

3. Drugs (eg, anticholinergics; narcotics)

4. Hydrops; empyema

5. Hyperalimentation

6. Neoplasm arising in head of pancreas, ampulla of Vater, or lower common bile duct (Courvoisier gallbladder) 
7. Pancreatitis obstructing Vaterian segment

8. Postvagotomy; postsurgical

9. Pregnancy

10. Prolonged fasting

\section{UNCOMMON}

1. Acromegaly

2. AIDS-related cholangiopathy (cytomegalovirus; Cryptosporidium)

3. Alcoholism

4. Bedridden patient with chronic illness

5. Chagas' disease

6. Kawasaki S.

7. Leptospirosis

8. Mucocele

9. Normal variant

\section{References}

1. Eisenberg RL: Gastrointestinal Radiology: A Pattern Approach. (ed 3) Philadelphia: Lippincott, 1996, pp 809-811

2. Gore RM: Gallbladder and biliary tract: Differential diagnosis. In: Gore RM, Levine MS: Textbook of Gastrointestinal Radiology. (ed 2) Philadelphia: WB Saunders, 2000, pp 1408-1414

3. Kapicioglu S, Gurbuz S, Danalioglu A, et al: Measurement of gallbladder volume with ultrasonography in pregnant women. Can J Gastroenterol 2000;14:403-405

4. Parulekar SG: Gallbladder and bile ducts. In: McGahan JP, Goldberg BB (eds): Diagnostic Ultrasound. Philadelphia: Lippincott-Raven, 1997, chapter 22

\section{Gamut G-111}

\section{DISTENDED GALLBLADDER IN A CHILD (US, CT)}

\section{COMMON}

1. Kawasaki S.

2. Total parenteral nutrition

\section{UNCOMMON}

1. Gallstone in cystic duct

2. Gastroenteritis
3. Leptospirosis

4. Normal variant

5. Scarlet fever

6. Upper respiratory infection

\section{References}

1. Donaldson JS: Diseases of the pediatric gallbladder. In: Gore RM, Levine MS (eds): Textbook of Gastrointestinal Radiology. (ed 2) Philadelphia: WB Saunders, 2000, pp 2140-2148

2. Gilger MA: Diseases of the gallbladder. In: Wyllie R, Hyams JS: Pediatric Gastrointestinal Disease. Philadelphia: WB Saunders, 1999

3. Parulekar SG: Gallbladder and bile ducts. In: McGahan JP, Goldberg BB (eds): Diagnostic Ultrasound. Philadelphia: Lippincott-Raven, 1997, chapter 22

4. Siegel MJ: Pediatric Body CT. Philadelphia: Lippincott Williams \& Wilkins, 1999

\section{Gamut G-112 \\ MULTISEPTATE GALLBLADDER (US, CT)}

1. Adenomyomatosis

2. Cholesterolosis

3. Congenital malformation

4. Desquamated gallbladder mucosa

5. Normal folded gallbladder (incl. Phrygian cap)

\section{References}

1. Chapman S, Nakielny R: Aids to Radiological Differential Diagnosis. London: Bailliere Tindall, 1990

2. Rumack CM, Nilson SR, Charboneau JW (eds): Diagnostic Ultrasound. (ed 2) St. Louis: Mosby, 1998

3. Gore RM: Gallbladder and biliary tract disease: Differential diagnosis. In: Gore RM, Levine MS (eds): Textbook of Gastrointestinal Radiology. (ed 2) Philadelphia: WB Saunders, 2000, pp 1408-1415

4. Gore RM, Fulcher AS, Taylor AJ, et al: Anomalies and anatomic variants of the gallbladder and biliary tract. In Gore RM, Levine MS (eds): Textbook of Gastrointestinal Radiology. (ed 2) Philadelphia: WB Saunders, 2000, pp 1305-1320 


\section{Gamut G-113}

\section{GALLBLADDER DISEASE SECONDARY TO CYSTIC DUCT OR INFUNDIBULUM OBSTRUCTION}

\section{COMMON}

1. Cholecystitis

2. Hydrops; empyema; mucocele of gallbladder

3. Milk of calcium bile

4. Porcelain gallbladder (calcified gallbladder wall)

\section{UNCOMMON}

1. Choledochoenteric fistula; gallstone ileus (eg, from gallstone perforation)

2. Emphysematous cholecystitis

3. Ruptured gallbladder

\section{Reference}

1. Zeman RK: Cholelithiasis and cholecystitis. In Gore RM, Levine MS (eds): Textbook of Gastrointestinal Radiology. (ed 2) Philadelphia: WB Saunders, 2000, pp 1321-1345

\section{Gamut G-114 \\ CALCIFICATION IN THE GALLBLADDER OR COMMON BILE DUCT}

\section{COMMON}

1. Gallstone (eg, calcium bilirubinate or calcium carbonate)

2. [Other right upper quadrant density (eg, stone in kidney or retrocecal appendix; calcified aneurysm; barium in diverticulum)]

\section{UNCOMMON}

1. Milk of calcium bile

2. Mucinous adenocarcinoma of gallbladder
3. Porcelain gallbladder

4. Schistosomiasis

5. Stone in common duct or cystic duct remnant

[ ] This condition does not actually cause the gamuted imaging finding, but can produce similar imaging changes that simulate it.

\section{References}

1. Eisenberg RL: Gastrointestinal Radiology: A Pattern Approach. (ed 3) Philadelphia: Lippincott, 1996, pp 10021007

2. Fatoar S, Al Ansari AG, Bassiony H, et al: Calcified pancreas and bile ducts from schistosomiasis. Br J Radiol 1996;59:1064-1066

\section{Gamut G-115-S1}

\section{ARTIFACTS THAT MIMIC GALLSTONES}

1. Inspissated sludge

2. Intraluminal defect, any cause (See G-116)

3. Partial volume artifact with duodenal impression

4. Refraction from folds in gallbladder neck

\section{References}

1. Rumack CM, Nilson SR, Charboneau JW (eds): Diagnostic Ultrasound. (ed 2) St. Louis: Mosby, 1998

2. Gore RM: Gallbladder and biliary tract disease: Differential diagnosis. In: Gore RM Levine MS (eds): Textbook of Gastrointestinal Radiology. (ed 2) Philadelphia: WB Saunders, 2000, pp 1408-1415

3. Zeman RK: Cholelithiasis and cholecystitis. In Gore RM, Levine MS (eds): Textbook of Gastrointestinal Radiology. (ed 2) Philadelphia: WB Saunders, 2000, pp 1321-1345

4. Palanduz A, Yalcin I, Tonguc E, et al: Sonographic assessment of ceftriaxane-associated biliary pseudolithiasis in children. J Clin Ultrasound 2000;28:166-168 


\section{Gamut G-115-S2 \\ STRUCTURES THAT \\ SONOGRAPHICALLY MIMIC \\ THE GALLBLADDER (US)}

1. Abscess (esp. Near ligamentum teres)

2. Choledochal cyst

3. Dilated cystic duct remnant

4. Fluid-filled duodenal bulb

5. Hepatic cyst

6. Omental cyst

7. Renal cyst

\section{Reference}

1. Gore RM: Gallbladder and biliary tract disease: Differential diagnosis. In Gore RM, Levine MS (eds): Textbook of Gastrointestinal Radiology. (ed 2) Philadelphia: WB Saunders, 2000, pp 1408-1415

\section{Gamut G-116 \\ FIXED POLYPOID LESION(S) OR FILLING DEFECT(S) IN THE GALLBLADDER (US, CT, MRI)}

\section{COMMON}

*1. Adenomyoma; adenomyomatosis; hyperplastic cholecystosis

*2. Cholesterol polyp

*3. Cholesterolosis ("strawberry" gallbladder)

*4. Gallstone, adherent

5. [Phrygian cap]

\section{UNCOMMON}

*1. Adenoma (incl. adenomatous polyp; villous adenoma; papilloma; fibroadenoma; cystadenoma)

*2. Cholecystitis glandularis proliferans

3. Congenital fold or septum

4. Cyst (epithelial; mucous retention)
5. Ectopic pancreatic or gastric tissue

*6. Hamartomas in Peutz-Jeghers S.

*7. Inflammatory polyp

8. Metachromatic leukodystrophy

*9. Metastasis (esp. melanoma)

10. Neoplasm, benign (eg, carcinoid; neurinoma; angioma)

11. Neoplasm, malignant (eg, adenocarcinoma; leiomyosarcoma)

*12. Parasitic granuloma (eg, Ascaris)

13. Postoperative defect

14. [Pseudodefect in neck of gallbladder; other pseudopolyps]

*15. Vascular lesion (eg, varix; aneurysm; tortuous artery)

16. Xanthogranulomatous cholecystitis

* May be multiple.

[ ] This condition does not actually cause the gamuted imaging finding, but can produce imaging changes that simulate it.

\section{References}

1. Berk RN, van der Vegt JH, Lichtenstein JE: The hyperplastic cholecystoses: Cholesterolosis and adenomyomatosis. Radiology 1983;146:593-601

2. Eisenberg RL: Gastrointestinal Radiology: A Pattern Approach. (ed 3) Philadelphia: Lippincott, 1996, pp 817-836

3. Lichtenstein JE: Adenomyomatosis and cholesterolosis: the "hyperplastic cholecystoses." In: Gore RM, Levine MS: Textbook of Gastrointestinal Radiology. (ed 2) Philadelphia: WB Saunders, 2000

4. Parra JA, Acinas O, Bueno J, et al: Xanthogranulomatous cholecystitis: clinical, sonographic, and CT findings in 26 patients. AJR 2000;174:979-983

5. Vogel T, Schumacher V, Saleh A, et al: Extraintestinal polyps in Peutz-Jeghers syndrome: presentation of four cases and review of the literature. Int J Colorectal Dis 2000;15:118-123 


\section{Gamut G-117}

\section{NONSHADOWING LESION IN THE GALLBLADDER (US)}

\section{COMMON}

1. Gallstone, nonshadowing or not in the transducer focal zone

2. Polyp (adenomatous or cholesterol)

3. Sludge

\section{UNCOMMON}

1. Adenomyomatosis

2. Carcinoma of gallbladder

3. Desquamated mucosa

4. Fibrinous debris

5. Hematoma

6. Inspissated pus

7. Metastasis

8. Parasites (eg, Ascaris; Clonorchis; Fasciola)

9. Precipitated contrast medium from ERCP

\section{References}

1. Abbitt PL: Ultrasound: A Pattern Approach. New York: McGraw-Hill, 1995

2. Filly RA, Allen B, Minton MJ, et al: In vitro investigation of the origin of echoes within biliary sludge. J Clin Ultrasound 1980;8:193-200

3. Gore RM: Gallbladder and biliary tract: Differential diagnosis. In: Gore RM, Levine MS: Textbook of Gastrointestinal Radiology. (ed 2) Philadelphia: WB Saunders, 2000, pp 1408-1414

4. Rumack CM, Nilson SR, Charboneau JW (eds): Diagnostic Ultrasound. (ed 2) St. Louis: Mosby, 1998

\section{Gamut G-118 \\ ECHO(ES) WITHIN \\ THE GALLBLADDER (US)}

\author{
COMMON \\ 1. Acute cholecystitis \\ 2. Calculus \\ 3. Fold in gallbladder wall \\ 4. Polyp (adenomatous) \\ 5. Sludge
}

\section{UNCOMMON}

1. AIDS-related cholangiopathy

2. Carcinoma of gallbladder

3. Clonorchiasis; fascioliasis

4. Ectopic pancreas or gastric mucosa

5. Emphysematous cholecystitis

6. Empyema of gallbladder

7. Feces (via fistula)

8. Food particles after cholecystojejunostomy

9. Gangrenous cholecystitis

10. Hemobilia

11. Hemorrhagic cholecystitis

12. Metastasis

13. Milk of calcium

14. Papilloma

15. Sarcoma of gallbladder

\section{References}

1. Gore RM: Gallbladder and biliary tract disease: Differential diagnosis. In: Gore RM, Levine MS (eds): Textbook of Gastrointestinal Radiology. (ed 2) Philadelphia: WB Saunders, 2000, pp 1408-1415

2. Kabaalio GB, Cubuk M, Senol U, et al: Fascioliasis: US, $\mathrm{CT}$, and MRI findings with new observations. Abdom Imaging 2000;25:400-404

3. Williamson MR: Abdominal ultrasound. In: Essentials of Ultrasound. Philadelphia: WB Saunders, 1996, p 87 


\section{Gamut G-119-1}

\section{ECHOGENIC BILE/GALLBLADDER SLUDGE (US); HIGH DENSITY BILE (CT)}

1. [Artifact (side lobe and slice thickness artifact on US; volume averaging of normal liver on CT)]

2. Cholelithiasis

3. Extrahepatic biliary obstruction

4. Hemolysis

5. Hemorrhage; hematobilia (esp. posttraumatic)

6. Inflammatory debris or pus

7. Milk of calcium

8. Prolonged fasting

9. Sickle cell disease; thalassemia

10. Vicarious excretion of contrast media (eg, renal failure; recent ERCP; occasionally normal)

[ ] This condition does not actually cause the gamuted imaging finding, but can produce imaging changes that simulate it.

\section{References}

1. Parulekar SG: Gallbladder and bile ducts. In: McGahan JP, Goldberg BB (eds): Diagnostic Ultrasound. Philadelphia: Lippincott-Raven, 1997, chapter 22

2. Rumack CM, Nilson SR, Charboneau JW (eds): Diagnostic Ultrasound. (ed 2) St. Louis: Mosby, 1998

3. Slone RM, Fisher AJ: Pocket Guide to Body CT Differential Diagnosis. New York: McGraw-Hill, 1999, p 230

4. Van Beers BE, Pringot JH: Imaging of cholelithiasis: helical CT. Abdom Imaging 2001;26:15-20

5. Zeman RK: Cholelithiasis and cholecystitis. In Gore RM, Levine MS (eds): Textbook of Gastrointestinal Radiology. (ed 2) Philadelphia: WB Saunders, 2000, pp 1321-1345

\section{Gamut G-119-2}

\section{GALLSTONE OR SLUDGE IN FETAL OR NEONATAL GALLBLADDER (US)}

1. Administration of parenteral nutrition and furosemide to premature infant

2. Biliary tree malformation

3. Cholestasis
4. Familial predisposition

5. Hemolysis (eg, Rh or ABO blood group alloimmunization; sickle cell disease; spherocytosis; thalassemia)

6. Hepatic dysfunction

7. Idiopathic

8. Methadone intake

9. Monochorionic twin after intrauterine death of its co-twin

10. Sepsis

\section{References}

1. Petrikoveky S, Klein V, Holsten N: Sludge in fetal gallbladder: natural history and neonatal outcome. Br J Radiol 1996; 69:1017-1018

2. Devonald KJ, Ellwood DA, Colditz PB: The variable appearance of fetal gallstones. J Ultrasound Med 1992; 11:579-585

3. Schweizer P, Lenz MP, Kirschner HJ: Pathogenesis and symptomatology of cholelithiasis in childhood. Dig Surg 2000;17:459-467

\section{Gamut G-120}

\section{INCREASED ATTENUATION OF GALLBLADDER LUMEN (CT)}

\section{COMMON}

1. Gallstones

2. Prior endoscopic retrograde cholangiopancreatography $(\mathrm{ERCP})$ or oral cholecystography

3. Sludge; debris

\section{UNCOMMON}

1. Empyema of gallbladder with pus

2. Feces (via fistula)

3. Food particles after cholecystojejunostomy

4. Hemobilia

5. Hemorrhagic cholecystitis

6. Hydrops of gallbladder

7. Milk of calcium bile

8. Mucinous adenocarcinoma of the gallbladder

9. Polyp or papilloma projecting into lumen 
10. Vicarious excretion of contrast medium

11. Volume averaging with adjacent structures

\section{References}

1. Gore RM: Gallbladder and biliary tract disease. In Gore RM, Levine MS (eds): Textbook of Gastrointestinal Radiology. (ed 2) Philadelphia: WB Saunders, 2000, pp 14081415

2. Van Beers BE, Pringot JH: Imaging of cholelithiasis: helical CT. Abdom Imaging 2001;26:15-20

\section{Gamut G-121 \\ HYPERECHOIC FOCUS IN THE GALLBLADDER WALL (US)}

\section{COMMON}

1. Adenomyomatosis, cholesterolosis ("hyperplastic cholecystoses")

2. Gallstone (embedded)

3. Polyp, cholesterol or other

\section{UNCOMMON}

1. Cholecystitis glandularis proliferans

2. Emphysematous cholecystitis

3. Microabscess

4. Rokitansky-Aschoff sinuses

\section{References}

1. Graif M, Horovitz A, Itzchak Y, et al: Hyperechoic foci in the gallbladder wall as a sign of microabscess formation or diverticula. Radiology 1984;152:781-784

2. Lichtenstein JE: Adenomyomatosis and cholesterolosis: the "hyperplastic cholecystoses." In: Gore RM, Levine MS: Textbook of Gastrointestinal Radiology. (ed 2) Philadelphia: WB Saunders, 2000, pp 1353-1359

\section{Gamut G-122}

\section{FOCAL THICKENING OF THE} GALLBLADDER WALL (> 3MM) (US, CT)

\section{COMMON}

1. Adenomyomatosis

2. Adherent gallstone or sludge

3. Carcinoma of gallbladder

4. Polyp, inflammatory

\section{UNCOMMON}

1. Benign neoplasm (eg, adenoma; carcinoid; papilloma)

2. Ectopic mucosa

3. Gangrenous or hemorrhagic cholecystitis

4. Hematoma

5. Metastasis (esp. melanoma)

6. Varices

\section{References}

1. Abbitt PL: Ultrasound: A Pattern Approach. New York: McGraw-Hill, 1995

2. Pandey M, Sood BP, Shukla RC, et al: Carcinoma of the gallbladder: role of sonography in diagnosis and staging. J Clin Ultrasound 2000;28:227-232

3. Parulekar SG: Gallbladder and bile ducts. In: McGahan JP, Goldberg BB (eds): Diagnostic Ultrasound. Philadelphia: Lippincott-Raven, 1997, chapter 22

4. Slone RM, Fisher AJ: Pocket Guide to Body CT Differential Diagnosis. New York: McGraw-Hill, 1999, p 229

5. Ward LM, Fulcher AS, Pereles FS, et al: Neoplasms of the gallbladder and biliary tract. In Gore RM, Levine MS (eds): Textbook of Gastrointestinal Radiology. (ed 2) Philadelphia: WB Saunders, 2000, pp 1360-1374 


\section{Gamut G-123}

\section{DIFFUSE THICKENING OF THE GALLBLADDER WALL (US, CT, MRI)}

\section{COMMON}

1. AIDS-related cholangiopathy (cytomegalovirus; Cryptosporidium)

2. Ascites with edema of gallbladder wall (eg, cirrhosis; renal failure; hypoalbuminemia; heart failure)

3. Carcinoma of gallbladder

4. Cholecystitis, acute or chronic, usually with cholelithiasis

5. Cirrhosis; schistosomiasis; liver failure

6. Hepatitis (viral or alcoholic)

7. Portal hypertension

8. Postprandial (physiolologic contraction); incomplete distension; inadequate fasting

9. Total parenteral nutrition

\section{UNCOMMON}

1. Adenomyomatosis; hyperplastic cholecystosis

2. Brucellosis

3. Extrahepatic portal vein obstruction (eg, pancreatitis; carcinoma of pancreas)

4. Folds in gallbladder wall

5. Gangrenous gallbladder

6. Graft-versus-host disease

7. Hemorrhagic cholecystitis

8. Infectious mononucleosis

9. Kaposi sarcoma (in AIDS)

10. Lymphatic obstruction at porta hepatis

11. Lymphoma

12. Multiple myeloma

13. Necrotizing enterocolitis in infants

14. Peptic ulcer adjacent to gallbladder

15. Pyelonephritis of right kidney

16. Sclerosing cholangitis

17. Torsion of gallbladder

18. Varices of gallbladder wall

19. Xanthogranulomatous cholecystitis
References

1. Abbitt PL: Ultrasound: A Pattern Approach. New York: McGraw-Hill, 1995

2. Cohen SM, Kurtz AB: Biliary sonography. Radiol Clin North Am 1991;29:1171-1192

3. Eisenberg RL: Gastrointestinal Radiology: A Pattern Approach. (ed 3) Philadelphia: Lippincott, 1996, pp 1067-1071

4. Fiske CE, Laing FC, Brown TW: Ultrasonographic evidence of gallbladder wall thickening in association with hypoalbuminemia. Radiology 1980;135:713-716

5. Gore RM: Gallbladder and biliary tract: Differential diagnosis. In: Gore RM, Levine MS: Textbook of Gastrointestinal Radiology. (ed 2) Philadelphia: WB Saunders, 2000, pp 1408-1414

6. Parulekar SG: Gallbladder and bile ducts. In: McGahan JP, Goldberg BB (eds): Diagnostic Ultrasound. Philadelphia: Lippincott-Raven, 1997, chapter 22

7. Ralls PW, Quinn MF, Juttner HU, et al: Gallbladder wall thickening: Patients without intrinsic gallbladder disease. AJR 1981;137:65-68

8. Sanders RC: The significance of sonographic gallbladder wall thickening. J Clin Ultrasound 1980;8:143-146

9. Shlaer WJ, Leopold GR, Scheible FW: Sonography of the thickened gallbladder wall: A nonspecific finding. AJR 1981;136:337-339

10. Williamson MR: Abdominal ultrasound. In: Essentials of Ultrasound. Philadelphia: WB Saunders, 1996, p 93

\section{Gamut G-124}

\section{STRIATIONS IN A THICKENED GALLBLADDER WALL (US, CT)}

\section{COMMON}

1. AIDS-related cholangiopathy (cytomegalovirus; Cryptosporidium)

2. Gangrenous cholecystitis

3. Non-biliary related edema or inflammation (eg, heart failure; renal failure; liver failure; hypoalbuminemia; ascites)

\section{UNCOMMON}

1. Adenomyomatosis

2. Blockage of lymphatic and venous drainage of gallbladder 
3. Hepatitis

4. Pancreatitis

5. Varices of gallbladder wall

6. Xanthogranulomatous cholecystitis

\section{References}

1. MacCarty RL: Inflammatory disorders of the biliary tract. In: Gore RM, Levine MS: Textbook of Gastrointestinal Radiology. (ed 2) Philadelphia: WB Saunders, 2000, pp 13751395

2. Teefey SA, Baron RL, Bigler SA: Sonography of the gallbladder: significance of striated (layered) thickening of the gallbladder wall. AJR 1991;156:945-951

3. Williamson MR: Abdominal Ultrasound. In: Essentials of Ultrasound. Philadelphia: WB Saunders, 1996, p 90

\section{Gamut G-125}

\section{EXTRINSIC DEFORMITY OR DISPLACEMENT OF THE GALLBLADDER (CAG, MRCP, CT)}

\section{COMMON}

1. Liver mass (eg, hepatocellular carcinoma; hemangioma; metastases; regenerating nodule; abscess; hydatid cyst; polycystic disease)

2. Normal duodenum or colon

\section{UNCOMMON}

1. Choledochal cyst

2. Duodenal mass (eg, neoplasm; hematoma)

3. Lymphadenopathy (eg, lymphoma $\mathrm{g}_{\mathrm{g}}$; metastases)

4. Pancreatic mass (eg, neoplasm; pseudocyst)

5. Retroperitoneal tumor or cyst (eg, renal, adrenal, soft tissue); polycystic kidney

\section{Reference}

1. Eisenberg RL: Gastrointestinal Radiology: A Pattern Approach. (ed 3) Philadelphia: Lippincott, 1996, pp 813-816

\section{Gamut G-126}

\section{PERICHOLECYSTIC FLUID ON ULTRASOUND}

\section{COMMON}

1. Acute cholecystitis (with or without perforation)

2. Ascites

\section{UNCOMMON}

1. AIDS-related cholangiopathy (cytomegalovirus; Cryptosporidium)

2. Hematoma

3. Pancreatitis

4. Peptic ulcer disease

5. Perforated appendix or diverticulum

6. Pericholecystic abscess

7. Peritonitis

8. Torsion of gallbladder

\section{References}

1. Rumack CM, Nilson SR, Charboneau JW (eds): Diagnostic Ultrasound. (ed 2) St. Louis: Mosby, 1998

2. Usui M, Matsuda S, Suzuki H, et al: Preoperative diagnosis of gallbladder torsion by magnetic resonance cholangiopancreatography. Scand J Gastroenterol 2000;35:218222

3. Williamson MR: Abdominal ultrasound. In: Essentials of Ultrasound. Philadelphia: WB Saunders, 1996, p 90

\section{Gamut G-127}

DELAYED VISUALIZATION OF THE GALLBLADDER ON SCINTIGRAPHY

\section{COMMON}

1. Chronic cholecystitis

\section{UNCOMMON}

1. Acalculus cholecystitis

2. Carcinoma of gallbladder

(continued) 
3. Dubin-Johnson syndrome

4. Hepatocellular disease (eg, cirrhosis; hepatitis)

5. Pancreatitis

6. Total parenteral nutrition

\section{Reference}

1. Gore RM: Gallbladder and biliary tract disease. In Gore RM, Levine MS (eds): Textbook of Gastrointestinal Radiology. (ed 2) Philadelphia: WB Saunders, 2000, pp 14081415

\section{Gamut G-128}

\section{GAS IN THE GALLBLADDER OR BILIARY TRACT}

\section{COMMON}

1. Biliary-enteric fistula to duodenum or colon (eg, perforated ulcer or adenocarcinoma of duodenum, ampulla, bile duct, gallbladder, stomach, pancreas, or colon; metastasis; lymphoma ${ }_{\mathrm{g}}$ ) (See G-139)

2. Cholecystitis with perforation

3. Emphysematous cholecystitis, cholangitis (esp. in diabetic)

4. [Gas in portal vein]

5. Postoperative (eg, sphincterotomy; biliary-intestinal anastomosis \{cholecystoenterostomy; choledochoenterostomy; Whipple procedure $\}$; internal or external biliary drainage)

6. Recent passage of gallstone from gallbladder or common duct (eg, gallstone ileus)

\section{UNCOMMON}

1. Common duct entry into duodenal diverticulum

2. Crohn's disease

3. [Gas in gallstone]

4. [Normal periductal fat]

5. Pancreatitis

6. Parasitic disease (clonorchiasis; ascariasis; ruptured amebic abscess of liver; strongyloidiasis of duodenum)

7. Peptic ulcer with perforation into common duct and fistula
8. Physiologic (incompetent, patulous sphincter, esp. in elderly)

9. Postbulbar duodenal ulcer adjacent to ampulla with spasm (acute) or fibrosis (healing)

10. Trauma, external penetrating or iatrogenic (eg, intubation; ERCP)

[ ] This condition does not actually cause the gamuted imaging finding, but can produce imaging changes that simulate it.

\section{References}

1. Eisenberg RL: Gastrointestinal Radiology: A Pattern Approach. (ed 3) Philadelphia: Lippincott, 1996, pp 888-893

2. Laing FC: The gallbladder and bile ducts. The liver. In: Rumack CM, Wilson SR, Charboneau JW (eds): Diagnostic Ultrasound. St. Louis: Mosby, 1998, pp 175-223

3. Martin DF, Tweedle DEF: The aetiology and significance of distal choledochoduodenal fistula. Br J Surg 1984;71: 632-634

4. Shimono T, Nishimura, Hayakawa K: CT imaging of biliary enteric fistula. Abdom Imaging 1998;23:172-176

5. Tamada K, Tomiyama T, Wada S, et al: Hyperechoic lines as a sonographic confirmatory sign during percutaneous transhepatic biliary drainage. Abdom Imaging 2001;26:3942

\section{Gamut G-129}

\section{CONGENITAL DISORDERS OR SYNDROMES WITH AN ABNORMAL BILIARY TRACT}

\section{COMMON}

1. Asplenia S. (Ivemark S.); polysplenia S.

2. Biliary atresia

3. Caroli disease

4. Choledochal cyst

5. Cystic fibrosis (mucoviscidosis)

\section{UNCOMMON}

1. Bardet-Biedl S.

2. Bile duct hypoplasia (Alagille S. or arteriohepatic S.)

3. Bile plug $\mathrm{S}$.

4. Hepatic fibrosis-renal cystic disease 
5. Meckel S.

6. Spontaneous (idiopathic) perforation of common bile duct

\section{References}

1. Arcemont CM, Meza MP, Arumanla S, et al: MRCP in the evaluation of pancreaticobiliary disease in children. Pediatr Radiology 2001;31:92-97

2. Nicotra JJ, Kramer SS, Bellah RD, Redd DCB: Congenital and acquired biliary disorders in children. Semin Roentgenol 1997;32:215-227

3. Park KB, Auh YH, Kim JH, et al: Diagnostic pitfalls in the cholangiographic diagnosis of choledochoceles: cholangiographic quality and its effect on visualization. Abdom Imaging 2001;26:48-54

4. Rosenthal P: Biliary atresia and neonatal disorders of the bile ducts. In: Wyllie R, Hyams JS: Pediatric Gastrointestinal Disease. Philadelphia: WB Saunders, 1999, pp 568-578

5. Siegel MJ: Pediatric Body CT. Philadelphia: Lippincott Williams \& Wilkins, 1999

6. Taybi H, Lachman RS: Radiology of Syndromes, Metabolic Disorders, and Skeletal Dysplasias. (ed 4) St. Louis: MosbyYear Book, 1996, p 962

\section{Gamut G-130 \\ DILATATION OF BILE DUCTS (CAG, MRCP, US, CT, MRI)}

\section{COMMON}

1. Advanced age

2. Calculus in biliary duct (choledocholithiasis)

3. Chronic pancreatitis

4. Lymphadenopathy with extrinsic compression

5. Neoplasm of pancreas, ampulla of Vater, common duct, or major bile duct (eg, papilloma; adenocarcinoma; mucin-producing cholangiocarcinoma)

6. Papillitis or fibrosis of ampulla of Vater

7. Parasitic disease (eg, ascariasis; clonorchiasis; opisthorchiasis; fascioliasis; hydatid disease)

8. Sclerosing cholangitis

9. Stricture of distal biliary duct (eg, postoperative; intubation; inflammatory; congenital)

\section{UNCOMMON}

1. Aneurysm of hepatic artery or aorta (compression)

2. Biliary atresia, extrahepatic

3. Biliary duct web or diaphragm

4. Caroli disease

5. Cholangitis, infectious (eg, AIDS-related; bacterial; parasitic)

6. Choledochal cyst

7. Choledochocele

8. Diverticulum of duodenum or biliary duct (compression)

9. Duodenal ulcer, penetrating

10. Hepatic fibrosis-renal cystic disease

11. Liver abscess (pyogenic, amebic, or fungal)

12. Liver infarcts following transcatheter embolization of hepatic artery branches

13. Lymphadenopathy in periportal area with ductal compression (eg, metastatic carcinoma of liver, stomach, or pancreas; lymphoma ${ }_{\mathrm{g}}$; sarcoidosis)

14. Metastasis

15. Mirizzi syndrome

16. Papillitis or fibrosis of ampulla

17. [Periportal edema]

18. Retroperitoneal fibrosis

[ ] This condition does not actually cause the gamuted imaging finding, but can produce imaging changes that simulate it.

\section{References}

1. Alexander ES, Mitchell SE: Dilatation of biliary ducts (extrahepatic, intrahepatic). Semin Roentgenol 1981;16:3-4

2. Berk RN, Clemett AR: Radiology of the Gallbladder and Bile Ducts. Philadelphia: WB Saunders, 1977

3. Eisenberg RL: Gastrointestinal Radiology: A Pattern Approach. (ed 3) Philadelphia: Lippincott, 1996, pp 872-881

4. Gore RM: Gallbladder and biliary tract: Differential diagnosis. In: Gore RM, Levine MS: Textbook of Gastrointestinal Radiology. (ed 2) Philadelphia: WB Saunders, 2000, pp 1408-1414

5. Lidofsky S, Scharschmidt BF: Jaundice. In: Feldman M, Scharschmidt BF, Sleisenger MH: Gastrointestinal and Liver Disease. (ed 6) Philadelphia: WB Saunders, 2000

6. Parulekar SG: Gallbladder and bile ducts. In: McGahan JP, Goldberg BB (eds): Diagnostic Ultrasound. Philadelphia: Lippincott-Raven, 1997, chapter 22

7. Slone RM, Fisher AJ: Pocket Guide to Body CT Differential Diagnosis. New York: McGraw-Hill, 1999, pp 226-227

8. Vitellas KM, Keogan MT, Spritzer CE, et al: MR cholangiopancreatography of bile and pancreatic duct abnormalities with emphasis on the single-shot fast spin-echo technique. RadioGraphics 2000;20:939-957 


\section{Gamut G-131}

\section{BILIARY DILATATION WITHOUT JAUNDICE OR OBSTRUCTION}

1. Advanced age

2. Choledochal cyst (type I)

3. Common duct exploration sequela

4. Early ductal obstruction

5. Nonobstructive gallstone

6. Normal variant

7. Parasitic disease (eg, Ascaris; Clonorchis; Fasciola; Opisthorchis; ruptured hydatid cyst or amebic abscess into duct)

8. Postcholecystectomy

9. Post-ductal obstruction

10. Recent passage of stone with ampullary edema

\section{Reference}

1. Gore RM: Gallbladder and biliary tract disease. In Gore RM, Levine MS (eds): Textbook of Gastrointestinal Radiology. (ed 2) Philadelphia: WB Saunders, 2000, pp 14081415

\section{Gamut G-132}

\section{BILIARY OBSTRUCTION WITHOUT DILATATION}

1. Acute severe biliary obstruction (first 3 days)

2. Cholangiocarcinoma with tumor encasement

3. Cholangitis (eg, ascending; sclerosing; recurrent pyogenic)

4. Hemobilia

5. Pancreatitis

6. Parasitic disease (eg, solitary or few Ascaris, Clonorchis, or Fasciola; ruptured hydatid cyst or amebic abscess into duct with debris)

\section{Reference}

1. Gore RM: Gallbladder and biliary tract disease. In Gore RM, Levine MS (eds): Textbook of Gastrointestinal Radiology. (ed 2) Philadelphia: WB Saunders, 2000, pp 1408-1415

\section{Gamut G-133-1}

\section{CYSTIC AND SACCULAR LESIONS OF THE BILE DUCTS-WITH NORMAL-SIZED INTRAHEPATIC BILE DUCTS (CAG, MRCP)}

1. Choledochal cyst (common duct)

2. Choledochocele (intraduodenal)

3. Cystic duct remnant

4. Diverticulum of common duct or rarely, an intrahepatic duct

5. Simple central dilatation of common duct

\section{References}

1. Burgener FA, Kormano M: Differential Diagnosis in Conventional Radiology. (ed 2) New York: Thieme Medical Publ, 1991, pp 730-731

2. DeBacker AI, Van den Abbeele K, DeSchopper AM: Choledochocele: diagnosis by magnetic resonance imaging. Abdom Imaging 2000;25:508-510

3. Gore RM, Fulcher AS, Taylor AJ: Anomalies and anatomic variants of the gallbladder and biliary tract. In: Gore RM, Levine MS: Textbook of Gastrointestinal Radiology. (ed 2) Philadelphia: WB Saunders, 2000

\section{Gamut G-133-2}

\section{CYSTIC AND SACCULAR LESIONS OF THE BILE DUCTS-WITH DILATATION OF INTRAHEPATIC BILE DUCTS (CAG, MRCP)}

1. Bacterial cholangitis with tiny saccular abscesses (acute, suppurative, ascending cholangitis)

2. Caroli disease

3. Choledochal cyst

4. Hepatic fibrosis-renal cystic disease

5. Parasitic disease (esp. opisthorchiasis; also clonorchiasis; ascariasis)

6. Recurrent pyogenic cholangitis (Oriental cholangiohepatitis) 
7. Reversible dilatation of intrahepatic bile ducts

8. Sclerosing cholangitis with prestenotic saccular outpouchings

\section{References}

1. Burgener FA, Kormano M: Differential Diagnosis in Conventional Radiology. (ed 2) New York: Thieme Medical Publ, 1991, pp 730-731

2. Donaldson JS: Diseases of the pediatric gallbladder and biliary tract. In: Gore RM, Levine MS: Textbook of Gastrointestinal Radiology. (ed 2) Philadelphia: WB Saunders, 2000

3. Jequier S, Capusten B, Guttman F, et al: Childhood choledochal cyst with intrahepatic enlarged cyst-like bile ducts. J Can Assoc Radiol 1984;35:73-76

4. Turner MA, Fulcher AS: The cystic duct: normal anatomy and disease processes. RadioGraphics 2001;21:3-22

\section{Gamut G-134}

\section{FILLING DEFECT OR SEGMENTAL LESION IN THE BILE DUCTS (CAG, MRCP, CT)}

\section{COMMON}

1. Air bubble

2. Calculus

3. Contraction of choledochal sphincter (pseudocalculus) in distal common duct

4. Edema of ampullary segment (eg, after passage of calculus; pancreatitis)

5. Extrinsic vascular impression (eg, right hepatic artery; bile duct varices)

6. Malignant neoplasm of bile duct (cholangiocarcinoma), gallbladder, ampulla, duodenum, or pancreas; hepatocellular carcinoma (hepatoma); Klatzkin tumor

7. Stricture (eg, cholangitis or Oriental cholangiohepatitis with dilated ducts and calculi)

\section{UNCOMMON}

1. Blood clot

2. Congenital membranous diaphragm (web) of common hepatic duct
3. Debris or mucus in ducts from obstructing tumor or parasites

4. Foreign body or food particle

5. Lymphadenopathy in porta hepatis

6. Metastasis (eg, lung; melanoma; lymphoma $_{\mathrm{g}}$ )

7. Mirizzi syndrome

8. Neoplasm, benign (eg, adenoma; papilloma; carcinoid; gastrointestinal stromal tumor ${ }_{\mathrm{g}}$; lipoma; hamartoma; polyp)

9. Normal variant (eg, cystic duct insertion; valves of Heister; redundant walls of tortuous duct)

10. Parasite (Ascaris; Clonorchis; Fasciola; hydatid cyst)

11. Pericholedochal adhesions

12. Postoperative defect (eg, plication defect at the site of duct-to-duct anastomosis)

13. Sarcoma botryoides (child)

14. Spasm of sphincter of Oddi

\section{References}

1. Eisenberg RL: Gastrointestinal Radiology: A Pattern Approach. (ed 3) Philadelphia: Lippincott, 1996, pp 837-871

2. Fulcher AS, Turner MA: Pitfalls of MR cholangiopancreatography. J Comput Assist Tomogr 1998;22:845-850

3. Gallix BP, Régent D, Bruel J-M: Use of magnetic resonance cholangiography in the diagnosis of choledocholithiasis. Abdom Imaging 2001;26:21-27

4. Pickuth D: Radiologic diagnosis of common bile duct stones. Abdom Imaging 2000;25:613-621

5. Vilgrain V, Palazzol L: Choledocholithiasis: role of US and endoscopic ultrasound. Abdom Imaging 2001:26:7-14 


\section{Gamut G-135}

\section{THICKENING OF BILE DUCT WALLS (US)}

\section{COMMON}

1. AIDS-related cholangiopathy

2. Ascending (bacterial) cholangitis

3. Choledocholithiasis

4. Pancreatitis (common duct)

5. Recurrent pyogenic cholangitis (Oriental cholangiohepatitis)

6. Sclerosing cholangitis

\section{UNCOMMON}

1. Cholangiocarcinoma

2. Parasitic disease (liver flukes \{clonorchiasis; opisthorchiasis \}; ascariasis; schistosomiasis; ruptured amebic hepatic abscess into bile ducts)

\section{References}

1. Berger J, Linsell DRM: Thickening of the walls of nondilated bile ducts. Clin Radiol 1997;52:474-476

2. Parulekar SG: Gallbladder and bile ducts. In: McGahan JP, Goldberg BB (eds): Diagnostic Ultrasound. Philadelphia: Lippincott-Raven, 1997, chapter 2

3. Vitellas KM, Keogan MT, Freed KS, et al: Radiologic manifestations of sclerosing cholangitis with emphasis on MR cholangiopancreatography. RadioGraphics 2000;20:959975

\section{Gamut G-136 \\ ECHOES WITHIN THE BILE DUCTS (US)}

\section{COMMON}
1. Calculus
2. Hemobilia
3. Pneumobilia
4. Pus
5. Sludge

\section{UNCOMMON}

1. Cholangiocarcinoma

2. Feces (via fistula)

3. Food particles (via reflux)

4. Hepatoma

5. Mesenchymal tumor, benign

6. Metastasis

7. Parasites (Ascaris; Clonorchis; Fasciola; hydatid debris)

8. Recurrent pyogenic cholangitis (Oriental cholangiohepatitis)

9. Surgical clips

\section{References}

1. Kabaalio GB, Cubuk M, Senol U, et al: Fascioliasis: US, $\mathrm{CT}$, and MRI findings with new observations. Abdom Imaging 2000;25:400-404

2. Williamson MR: Abdominal ultrasound. In: Essentials of Ultrasound. Philadelphia: WB Saunders, 1996, p 77

\section{Gamut G-137 BILE DUCT NARROWING OR
OBSTRUCTION (CAG, MRCP)}

\section{COMMON}

1. Calculus in biliary duct (choledocholithiasis) (eg, impacted stone in Vaterian segment; papillary edema from recent passage of stone; Mirizzi syndrome)

2. Cholangitis

3. Contraction of choledochal sphincter (pseudocalculus); papillary stenosis

4. Iatrogenic or posttraumatic (eg, surgical injury; trauma; radiation therapy; hepatic artery chemotherapy or embolization)

5. Neoplasm, malignant (eg, cholangiocarcinoma; ampullary, pancreatic, duodenal, or gallbladder carcinoma; hepatocellular carcinoma; Klatzkin tumor; villous tumor)

6. Pancreatitis, acute or chronic (incl. pseudocyst)

7. Sclerosing cholangitis 


\section{UNCOMMON}

1. Abscess (pyogenic, amebic, or fungal)

2. AIDS-related cholangiopathy (eg, cytomegalovirus or Cryptosporidium infection)

3. Artifact from post-processing technique of MRCP

4. Biliary hypoplasia or atresia

5. Caroli disease (complicated)

6. Congenital membranous diaphragm (web)

7. Debris or mucus in ducts from obstructing tumor or parasites

8. Duodenal diverticulum

9. Hepatic fibrosis-renal cystic disease

10. Hepatocellular disease, advanced (eg, cirrhosis; cholangiolytic hepatitis)

11. Liver cyst, neoplasm, or abscess

12. Lymphadenopathy in porta hepatis (eg, metastasis; lymphoma $_{\mathrm{g}}$; tuberculosis; sarcoidosis)

13. Metastasis (esp. from carcinoma of pancreas, gallbladder, stomach)

14. Neoplasm, benign (incl. adenoma; papilloma; gastrointestinal stromal tumor ${ }_{\mathrm{g}}$; myoblastoma; cystadenoma)

15. Papillitis of ampulla

16. Parasitic disease of bile ducts, liver, or duodenum (esp. ascariasis; clonorchiasis; fascioliasis; hydatid disease; amebic abscess; schistosomiasis; strongyloidiasis)

17. Postbulbar duodenal ulcer with scarring or perforation

18. Recurrent pyogenic cholangitis \{Oriental cholangiohepatitis

19. Stricture, traumatic or iatrogenic (eg, postoperative; intubation)

20. Vascular compression (eg, aneurysm of aorta or hepatic artery; calcified portal vein)

\section{References}

1. Dähnert W: Radiology Review Manual. Baltimore: Williams \& Wilkins, 1999

2. Chapman S, Nakielny R: Aids to Radiological Differential Diagnosis. London: Bailliere Tindall, 1990

3. Eisenberg RL: Gastrointestinal Radiology: A Pattern Approach. (ed 3) Philadelphia: Lippincott, 1996, pp 851-871

4. Fulcher AS, Turner MA: Pitfalls of MR cholangiopancreatography. J Comput Assist Tomogr 1998;22:845-850
5. Gore RM: Gallbladder and biliary tract: Differential diagnosis. In: Gore RM, Levine MS: Textbook of Gastrointestinal Radiology. (ed 2) Philadelphia: WB Saunders, 2000, pp 1408-1414

6. Jacobson IM: ERCP and Its Applications. Philadelphia: Lippincott-Raven, 1998

7. Pavone P, Laghi A, Passariello R: MR cholangiopancreatography in malignant biliary obstruction. Semin Ultrasound CT MR 1999;20:317-323

8. Rajaram R, Ponsiden CY, Majoie CBLM, et al: Evaluation of a modified cholangiographic classification system for primary sclerosing cholangitis. Abdom Imaging 2001;26: 43-47

9. Semelka RC, Ascher SM, Reinhold C: MRI of the Abdomen and Pelvis. New York: Wiley-Liss, 1997

\section{Gamut G-138}

\section{ENLARGED PAPILLA OF VATER}

\section{COMMON}

1. Calculus impacted in distal common duct

2. Carcinoma of Vaterian segment

3. Idiopathic; normal variant

4. Mucinous (ductectatic) adenocarcinoma of pancreas

5. Pancreatitis

6. Papillitis

\section{UNCOMMON}

1. Edema secondary to active duodenal ulcer

2. Heterotopic pancreatic tissue

3. Neoplasm (eg, adenomatous polyp of papilla; carcinoid; gastrointestinal stromal tumor ${ }_{\mathrm{g}}$ of duodenum)

4. Pancreatic abscess

5. Parasitic disease (eg, strongyloidiasis; ascariasis)

6. Postoperative; instrumentation

7. Zollinger-Ellison syndrome

\section{References}

1. Eisenberg RL: Gastrointestinal Radiology: A Pattern Approach. (ed 3) Philadelphia: Lippincott, 1996, pp 882-887

2. Poppel MH, Jacobson HG, Smith RW: The Roentgen Aspects of the Papilla and Ampulla of Vater. Springfield, IL: CC Thomas, 1953 
3. Schutz SM: Papillary tumors. In: DiMarino AJ, Benjamin SB: Gastrointestinal Disease: An Endoscopic Approach. Malden, MA: Blackwell Science, 1997, pp 952-960

\section{Gamut G-139}

\section{BILIARY-ENTERIC FISTULA (See G-128)}

\section{COMMON}

1. Cholecystitis (perforative, acute or chronic)

2. Gallstone fistula from gallbladder or bile duct (eg, choledochoduodenal fistula)

3. Malignant neoplasm of gallbladder, bile duct, pancreas, or intestine

4. Postoperative (eg, sphincterotomy; biliary-intestinal anastomosis-Whipple procedure)

\section{UNCOMMON}

1. Abscess (pancreatic; hepatic; pericolic; pericholecystic)

2. Common duct entry into duodenal diverticulum

3. Diverticulitis of duodenum or hepatic flexure of colon

4. Granulomatous disease of duodenum or colon (eg, Crohn's disease; tuberculosis; actinomycosis)

5. Lymphoma

6. Parasitic disease (esp. amebiasis; ascariasis; hydatid disease)

7. Passage of common duct stone

8. Peptic ulcer perforation into biliary tract

9. [Physiologic reflux at ampulla; incompetent sphincter]

10. Trauma, external or iatrogenic (eg, intubation; ERCP)

[ ] This condition does not actually cause the gamuted imaging finding, but can produce imaging changes that simulate it.

\section{References}

1. Martin DF, Tweedle DEF: The aetiology and significance of distal choledochoduodenal fistula. Br J Surg 1984;71:632634
2. Zuckerman AM, Goldschmid S, Hunter JG, et al: Biliary fistulas. In: Pitt HA, Carr-Locke DL, Ferrucci JT, et al: Hepatobiliary and Pancreatic Disease. Boston: Little, Brown and Company, 1995

\section{Gamut G-140 BILIARY-PLEURAL (OR BRONCHIAL) FISTULA}

1. Biliary obstruction

2. Congenital

3. Parasitic disease (eg, amebic abscess; hydatid disease)

4. Trauma, external or iatrogenic

\section{References}

1. Bamberger PK, Stojadinovic A, Shaked G, et al: Biliarypleural fistula presenting as a massive pleural effusion after thoracoabdominal penetrating trauma. J Trauma 1997;43: 162-163

2. Böni RAH, Peter J, Marincek B: Amebic abscess of the liver manifested by "hemoptysis": US, CT, and MRI findings. Abdom Imag 1995;20:214-216

3. Feld R, Wechsler RJ, Bonn J: Biliary-pleural fistula without biliary obstruction. AJR 1997;169:381-383

4. Palmer PES, Reeder MM: The Imaging of Tropical Diseases. Heidelberg: Springer-Verlag, 2001

\section{Gamut G-141-1 \\ HEPATOMEGALY (See G-141-2)}

\section{COMMON}

1. Abscess, solitary or multiple (pyogenic, amebic, or fungal)

2. Cirrhosis, early

3. Congenital hepatomegaly (See G-141-2)

4. Cyst (bile duct cyst; simple; posttraumatic; hydatid) (See G-157) 
5. Elevated venous pressure (eg, heart failure; constrictive pericarditis; tricuspid stenosis or insufficiency)

6. Fatty change (steatosis) (See G-144)

7. Hemochromatosis

8. Hepatitis (viral, infectious, or serum)

9. Infectious disease, other (eg, infectious mononucleosis; candidiasis; brucellosis; miliary tuberculosis or histoplasmosis; malaria)

10. Metastases

11. Neoplasm (esp. hepatocellular carcinoma \{hepatoma\}; cholangiocarcinoma; hepatoblastoma; giant hemangioma; hemangioendothelioma; angiosarcoma)

12. Obstruction of common bile duct (biliary cirrhosis) (See G-137)

\section{UNCOMMON}

1. Amyloidosis

2. Anemia, primary (eg, thalassemia major)

3. Chronic granulomatous disease of childhood

4. Extramedullary hematopoiesis

5. Gaucher disease; Niemann-Pick disease

6. Glycogen storage disease (eg, type I-von Gierke)

7. Hematoma

8. Hydatid disease (Echinococcus granulosus and E. multilocularis)

9. Kala-azar

10. Langerhans cell histiocytosis ${ }_{\mathrm{g}}$

11. Lymphoma ${ }_{\mathrm{g}}$

12. Myeloid metaplasia; myelofibrosis

13. Polycystic disease of liver

14. Polycythemia vera

15. Reye S.

16. Sarcoidosis

17. Schistosomiasis

18. Thrombosis of hepatic vein or upper inferior cava (Budd-Chiari S.); veno-occlusive disease (See G-189)

19. Wilson disease

\section{Reference}

1. Lefkowitch JH: Pathologic diagnosis of liver disease. In: Zakim D, Boyer TD: Hepatology. (ed 3) Philadelphia: WB Saunders, 1996, pp 844-874

\section{Gamut G-141-2}

\section{CONGENITAL HEPATOMEGALY}

\section{COMMON}

1. Anemia ${ }_{\mathrm{g}}$, primary (esp. thalassemia; sickle cell disease)

2. Cystic fibrosis (mucoviscidosis)

3. Gaucher disease; Niemann-Pick disease

4. Glycogen storage disease, types I (von Gierke), III and IV

5. Infant of diabetic mother

6. Langerhans cell histiocytosis ${ }_{\mathrm{g}}$

7. Mucopolysaccharidoses (esp. Hurler S., Hunter S.); mucolipidosis II (I-cell disease) (See J-4)

8. Polycystic disease of liver

\section{UNCOMMON}

1. Aase $\mathrm{S}$.

2. Alagille S. (arteriohepatic S.)

3. Alpha-1-antitrypsin deficiency

4. Beckwith-Wiedemann S.

5. Budd-Chiari S.

6. Chédiak-Higashi S.

7. Cholesterol ester storage disease

8. Chronic granulomatous disease of childhood

9. Congenital transplacental infection (eg, toxoplasmosis; rubella; cytomegalovirus; herpes simplex)

10. Cystinosis

11. Ethanolaminosis

12. Farber disease (lipogranulomatosis)

13. Felty S.

14. Galactosemia

15. Galactosialidosis

16. $\mathrm{GM}_{1}$ gangliosidosis; fucosidosis; mannosidosis

17. Hepatic fibrosis-renal cystic disease

18. Homocystinuria

19. Hyperlipoproteinemia

20. Infantile multisystem inflammatory disease (NOMID)

21. Lipoatrophic diabetes

22. Mauriac S.

23. Osteopetrosis

(continued) 


\section{POEMS S.}

25. Pyruvate kinase deficiency

26. Sea-blue histiocyte $S$.

27. Tyrosinemia (type I)

28. Weber-Christian S.

29. Wilson disease

30. Wolman disease (familial xanthomatosis)

31. Zellweger S. (cerebrohepatorenal S.)

\section{References}

1. Siafakis CG, Jonas MM: Neonatal hepatitis. In: Wyllie R, Hyams JS: Pediatric Gastrointestinal Disease. Philadelphia: WB Saunders, 1999, pp 563-567

2. Siegel MJ: Pediatric Body CT. Philadelphia: Lippincott, Williams \& Wilkins, 1999

3. Taybi H, Lachman RS: Radiology of Syndromes, Metabolic Disorders, and Skeletal Dysplasias. (ed 4) St. Louis: MosbyYear Book, 1996, p 959

\section{Gamut G-142 DIFFUSE HEPATIC CALCIFICATIONS (PF, US, CT)}

\section{COMMON}

1. Hyperparathyroidism, secondary (incl. chronic renal failure; uremia; hemodialysis)

2. Ischemia, with or without shock; infarction (chronic)

3. Metastatic disease (eg, mucinous adenocarcinoma of colon, stomach, breast, or thyroid; carcinoma of ovary, pancreas, or lung; islet cell carcinoma; melanoma; neuroblastoma; osteosarcoma; chondrosarcoma; teratoma; also postradiation and postchemotherapy)

4. [Neoplasm of liver, large, advanced, often necrotic (eg, hepatocellular carcinoma; cholangiocarcinoma; hemangioma; infantile hemangioendothelioma; hepatoblastoma; fibrolamellar carcinoma; mesenchymal hamartoma) — calcifications may be extensive in a localized area or even multifocal, but not truly diffuse]

\section{UNCOMMON}

1. Amyloidosis

2. Chronic granulomatous disease of childhood

3. Congenital transplacental infection (eg, toxopolasmosis; rubella; cytomegalovirus; herpes simplex; varicella)

4. Granulomas, multiple healed (eg, tuberculosis; histoplasmosis; coccidioidomycosis; brucellosis)

5. Hemochromatosis

6. Hydatid disease (Echinococcus multilocularis or multiple calcified E. granulosus cysts)

7. Infection, severe (eg, multiple healed abscesses)

8. Pentastomiasis (Armillifer infection)

9. [Peritoneal calcifications overlying liver capsule in infancy (eg, meconium peritonitis; ruptured hydrometrocolpos)]

10. Schistosomiasis (esp. S. japonica)

11. [Thorotrast residual]

[ ] This condition does not actually cause the gamuted imaging finding, but can produce imaging changes that simulate it.

\section{References}

1. Gore RM: Liver: Differential diagnosis. In: Gore RM, Levine MS: Textbook of Gastrointestinal Radiology. (ed 2) Philadelphia: WB Saunders, 2000, pp 1712-1727

2. Kennan NM, Evans C: Hepatic and splenic calcification due to amyloid. Clin Radiol 1991;44:60-61

3. Milstein MJ, Moulton JS: Diffuse hepatic calcification after ischemic liver injury in a patient with chronic renal failure. AJR 1993;161:75-76

4. Parulekar SG, Bree RL: Liver. In: McGahan JP, Goldberg BB (eds): Diagnostic Ultrasound. Philadelphia: LippincottRaven, 1997, chapter 21 


\section{Gamut G-143}

\section{FETAL OR NEONATAL LIVER CALCIFICATION}

\section{COMMON}

1. Congenital transplacental infection (eg, toxopolasmosis; rubella; cytomegalovirus; herpes simplex; varicella)

2. Metastatic neuroblastoma

3. [Peritoneal calcifications overlying liver capsule (eg, meconium peritonitis; ruptured hydrometrocolpos)]

\section{UNCOMMON}

1. Infarcts

2. Primary liver tumor (eg, hemangioma; hepatoblastoma; infantile hemangioendothelioma; hamartoma; teratoma)

3. Thromboemboli in portal vein [or IVC]

[ ] This condition does not actually cause the gamuted imaging finding, but can produce imaging changes that simulate it.

\section{References}

1. Brugman SM. Bjelland JJ, Thomason JE, et al: Sonographic findings with radiologic correlation in meconium peritonitis. J Clin Ultrasound 1979;7:305-306

2. Chapman S, Nakielny R: Aids to Radiological Differential Diagnosis. (ed 3) London: WB Saunders, 1995, p 267

3. Friedman AP, Haller JO, Boyer B, Cooper R: Calcified portal vein thromboemboli in infants: radiography and ultrasonography. Radiology 1981;140:381-382

4. Nguyen DL, Leonard JC: Ischaemic hepatic necrosis: a cause of fetal liver calcification. AJR 1986:147:596-597

5. Schackelford GD, Kirks DR: Neonatal hepatic calcification secondary to transplacental infection. Radiology 1977;122: 753-757

6. Siegel MJ: Pediatric Body CT. Philadelphia: Lippincott, Williams \& Wilkins, 1999

\section{Gamut G-144}

\section{FATTY CHANGE IN THE LIVER (STEATOSIS) (US, CT, MRI)}

\author{
COMMON \\ *1. AIDS \\ 2. Alcoholism; cirrhosis \\ *3. Cystic fibrosis (mucoviscidosis) \\ 4. Diabetes mellitus \\ *5. Drug therapy (esp. tetracycline; steroids; \\ chemotherapy with cytotoxic agents) \\ *6. Idiopathic \\ *7. Obesity
}

\section{UNCOMMON}

1. Carbon tetrachloride exposure

*2. Cushing S.

*3. Fever, prolonged

*4. Hepatitis, acute or viral

*5. Hepatotoxins

6. Hyperalimentation

7. Hyperlipidemia, familial

8. Jejunoileal bypass

*9. Lipoatrophic diabetes (congenital total lipodystrophy)

*10. Malabsorption syndrome (See G-57)

11. Peritoneal dialysis

12. Pregnancy

*13. Reye S.

*14. Starvation, acute or chronic (incl. malnutrition; kwashiorkor)

*15. Storage diseases (eg, Gaucher disease; NiemannPick disease; glycogen storage disease, type Ivon Gierke)

* Children affected.

\section{References}

1. Baron RL, Gore RM: Diffuse liver disease. In: Gore RM, Levine MS: Textbook of Gastrointestinal Radiology. (ed 2) Philadelphia: WB Saunders, 2000, pp 1590-1638 
2. Bashist B, Hecht HL, Harley WD: Computed tomographic demonstration of rapid changes in fatty infiltration of the liver. Radiology 1982;142:691-692

3. Taybi H, Lachman RS: Radiology of Syndromes, Metabolic Disorders, and Skeletal Dysplasias. (ed 4) St. Louis: MosbyYear Book, 1996

4. Wilson SR, Rosen IE, Chin-Sang HB, et al: Fatty infiltration of the liver: an imaging challenge. J Can Assoc Radiol $1982 ; 33: 227-232$

\section{Gamut G-145}

\section{PERFUSION ABNORMALITIES OF THE LIVER}

\section{LOBAR OR SEGMENTAL}

1. Cirrhosis with arterial-portal shunt

2. Hypervascular gallbladder disease

3. Mass effect due to tumor, cyst, abscess within liver

4. Portal vein ligation, obstruction or thrombosis

\section{SUBSEGMENTAL}

1. Acute cholecystitis

2. Ethanol ablation

3. Obstruction of peripheral portal branches

4. Percutaneous needle biopsy

\section{References}

1. Gore RM: Liver: Differential diagnosis. In: Gore RM, Levine MS (eds): Textbook of Gastrointestinal Radiology. (ed 2) Philadelphia: WB Saunders, 2000, pp 1712-1727

2. Gore RM, Marn CS, Baron RL: Vascular disorders of the liver and splanchnic circulation. In: Gore RM, Levine MS (eds): Textbook of Gastrointestinal Radiology. (ed 2) Philadelphia: WB Saunders, 2000, pp 1639-1668

3. Gabata T, Kadoya M, Matsui O, et al: Dynamic CT of hepatic abscess: significance of transient segmental enhancement. AJR 2001;176:675-680

4. Rozeik PE, Huppert PE, Münch H: Angiographic pseudolesion in the liver in asymptomatic subacute cholecystitis. Eur Radiol 2001;11:346-347

5. Semelka RC, Chung J-J, Hussain SM, et al: Chronic hepatitis: correlation of early patchy and late enhancement patterns on gadolinium-enhanced MR images with histopathology. JMRI 2001;385-391

\section{Gamut G-146}

\section{PATCHY HEPATOGRAM (AREAS OF LOW DENSITY ON ANGIOGRAPHY OR POSTCONTRAST CT)}

\section{COMMON}

1. Budd-Chiari syndrome

2. Cirrhosis

3. Heart failure

4. Hepatitis

5. Portal vein thrombosis

\section{UNCOMMON}

1. Lymphomatous infiltration

2. Sarcoidosis

3. Schistosomiasis

4. Thyrotoxicosis

5. Tricuspid atresia

\section{References}

1. Gore RM: Liver: Differential diagnosis. In: Gore RM, Levine MS (eds): Textbook of Gastrointestinal Radiology. (ed 2) Philadelphia: WB Saunders, 2000, pp 1712-1727

2. Gore RM, Marn CS, Baron RL: Vascular disorders of the liver and splanchnic circulation. In: Gore RM, Levine MS (eds): Textbook of Gastrointestinal Radiology. (ed 2) Philadelphia: WB Saunders, 2000, pp 1639-1668

3. Gabata T, Kadoya M, Matsui O, et al: Dynamic CT of hepatic abscess: significance of transient segmental enhancement. AJR 2001;176:675-680

4. Rozeik PE, Huppert PE, Münch H: Angiographic pseudolesion in the liver in asymptomatic subacute cholecystitis. Eur Radiol 2001;11:346-347

5. Semelka RC, Chung J-J, Hussain SM, et al: Chronic hepatitis: correlation of early patchy and late enhancement patterns on gadolinium-enhanced MR images with histopathology. JMRI 2001;385-391 


\section{Gamut G-147}

\section{GENERALIZED OR MULTIFOCAL DECREASED ECHOGENICITY OF THE LIVER ON ULTRASOUND (HYPOECHOIC)}

\section{COMMON}

1. Hepatitis, acute viral

2. Malignant infiltration of liver by primary or metastatic neoplasm

3. Schistosomiasis, early

\section{UNCOMMON}

1. Amyloidosis

2. Leukemia; lymphoma ${ }_{\mathrm{g} ;}$ Burkitt lymphoma

3. [Renal disease, end stage; nephrocalcinosis]

[ ] This condition does not actually cause the gamuted imaging finding, but can produce imaging changes that simulate it.

\section{References}

1. Baron RL, Gore RM: Diffuse liver disease. In: Gore RM, Levine MS: Textbook of Gastrointestinal Radiology. (ed 2) Philadelphia: WB Saunders, 2000, pp 1590-1638

2. Cosgrove DO: Liver and biliary tree. In: Barnett E, Morley P (eds): Clinical Diagnostic Ultrasound. Oxford: Blackwell Scientific Publ, 1985, pp 365-386

3. Gore RM: Liver: Differential diagnosis. In: Gore RM, Levine MS: Textbook of Gastrointestinal Radiology. (ed 2) Philadelphia: WB Saunders, 2000, pp 1712-1726

4. Konno K, Ishida H, Sato M, et al: Macronodular hepatic deformity on normal liver. Abdom Imaging 2000;25:592-595

5. Skolnick ML: Guide to the Ultrasound Examination of the Abdomen. New York: Springer-Verlag, 1986, pp 87-88

6. Weill FS: Ultrasound Diagnosis of Digestive Diseases. (ed 3 revised) Berlin: Springer-Verlag, 1990, pp 239-246

\section{Gamut G-148}

\section{GENERALIZED OR MULTIFOCAL INCREASED ECHOGENICITY OF THE LIVER ON ULTRASOUND (HYPERECHOIC)}

\section{COMMON}

1. AIDS

2. Fatty infiltration (eg, alcoholism; various toxins; diabetes; malabsorption S.; jejunoileal bypass; protein deficiency; starvation-malnutrition, kwashiorkor; familial hyperlipidemia) (See G-144)

3. Fibrosis of liver parenchyma (eg, alcoholism; cirrhosis; schistosomiasis; chronic hepatitis; glycogen storage disease)

4. Hepatocellular carcinoma (hepatoma), diffuse

5. Hydatid disease (Echinococcosis multilocularis or multiple healed, calcified $E$. granulosus cysts)

6. Idiopathic

7. Lipoatrophic diabetes

8. Obesity

9. Technical—excessive gain

\section{UNCOMMON}

1. Budd-Chiari S. (focal)

2. Carbon tetrachloride exposure

3. Cystic fibrosis (mucoviscidosis)

4. Drug therapy (esp. tetracycline; steroids; chemotherapy)

5. Gaucher disease

6. Hyperalimentation

7. Lipoatrophic diabetes (congenital total lipodystrophy)

8. Lymphoma ; $_{\text {;eukemia }}$

9. Miliary tuberculosis

10. Pregnancy

11. Reye S.

12. Tyrosinemia

13. Wilson disease

\section{References}

1. Baron RL, Gore RM: Diffuse Liver Disease. In: Gore RM, Levine MS: Textbook of Gastrointestinal Radiology. (ed 2) Philadelphia: WB Saunders, 2000, pp 1590-1638

(continued) 
2. Cosgrove DO: Liver and biliary tree. In: Barnett E, Morley P (eds): Clinical Diagnostic Ultrasound. Oxford: Blackwell Scientific Publ, 1985, pp 365-386

3. Skolnick ML: Guide to the Ultrasound Examination of the Abdomen. New York: Springer-Verlag, 1986, pp 87-88

4. Taybi H, Lachman RS: Radiology of Syndromes, Metabolic Disorders, and Skeletal Dysplasias. (ed 4) St. Louis: MosbyYear Book, 1996, pp 959-960

5. Weill FS: Ultrasound Diagnosis of Digestive Diseases. (ed 3 revised) Berlin: Springer-Verlag, 1990, pp 239-246

6. Williamson, MR: Abdominal ultrasound. In: Essentials of Ultrasound. Philadelphia: WB Saunders, 1996, p 79

7. Wilson SR, Rosen IE, Chin-Sang HB, et al: Fatty infiltration of the liver: an imaging challenge. J Can Assoc Radiol 1982;33:227-232

8. Zweibel WJ: Sonographic diagnosis of diffuse liver disease. Semin Ultrasound CT and MRI 1995;16:8-15

\section{Gamut G-149}

\section{GENERALIZED HIGH DENSITY LIVER (NONENHANCED CT)}

\section{COMMON}

1. Hemochromatosis

2. Hemosiderosis

\section{UNCOMMON}

1. Chronic arsenic poisoning

2. Drug therapy (eg, amiodarone; gold; cisplatin)

3. Glycogen storage disease, type I-von Gierke (may be low density liver)

4. Iron overload of liver (eg, multiple transfusions)

5. Storage diseases (usually low density)

6. Thorotrast

7. Wilson disease

\section{References}

1. Bacon BR: Hemochromatosis: diagnosis and management. Gastroenterology 2001;120:718-725

2. Butler S, Smathers RL: Computed tomography of amiodarone pulmonary toxicity. J Comput Assist Tomogr 1985; 9:375-376
3. Foley WD, Jochem RJ: Computed tomography: focal and diffuse liver masses. Radiol Clin North Am 1991;20:1213

4. Gore RM: Liver: Differential diagnosis. In: Gore RM, Levine MS: Textbook of Gastrointestinal Radiology. (ed 2) Philadelphia: WB Saunders, 2000, pp 1712-1727

5. Rofsky NM, Fleishaker H: CT and MRI of diffuse liver disease. Semin Ultrasound CT and MRI 1995;16:33

\section{Gamut G-150}

\section{GENERALIZED OR MULTIFOCAL LOW DENSITY LIVER (NONENHANCED CT)}

\section{COMMON}

1. Diffuse malignancy, primary or metastatic (incl. lymphoma)

2. Fatty infiltration (steatosis) (See G-144)

3. Hepatic congestion (eg, heart failure; constrictive pericarditis; tricuspid stenosis or insufficiency)

\section{UNCOMMON}

1. Amyloidosis

2. Budd-Chiari syndrome, acute or chronic

3. Cysts, numerous (eg, hydatid disease; Caroli disease; polycystic liver disease; Von Hippel-Lindau disease)

4. Storage diseases (eg, Gaucher disease; NiemannPick disease; glycogen storage disease, type Ivon Gierke)

\section{References}

1. Baron RL, Gore RM: Diffuse Liver Disease. In: Gore RM, Levine MS: Textbook of Gastrointestinal Radiology. (ed 2) Philadelphia: WB Saunders, 2000, pp 1590-1638

2. Foley WD, Jochem RJ: Computed tomography: focal and diffuse liver masses. Radiol Clin North Am 1991;20:1213

3. Gore RM: Liver: Differential diagnosis. In: Gore RM, Levine MS: Textbook of Gastrointestinal Radiology. (ed 2) Philadelphia: WB Saunders, 2000, pp 1712-1727

4. Halvorsen RA, Korobkin M, Ram PC, Thompson WM: CT appearance of focal fatty infiltration of the liver. AJR 1982;139:277-281

5. Mergo PJ, Ros PR: Benign lesions of the liver. Radiol Clin North Am 36:319-332, 1998 
6. Siegel MJ: Pediatric Body CT. Philadelphia: Lippincott Williams \& Wilkins, 1999

7. Slone RM, Fisher AJ: Pocket Guide to Body CT Differential Diagnosis. New York: McGraw-Hill, 1999, pp 203-204

8. Suzuki S, et al: CT findings in hepatic and splenic amyloidosis. J Comput Assist Tomog 1986;10:332-334

9. Vogelzang RL, Anscheutz SL, Gore RM: Budd Chiari syndrome: CT observations. Radiology 1987;163:329-333

10. Yates CK, Streight RA: Focal fatty infiltration of the liver simulating metastatic disease. Radiology 1986;159:83-84

\section{Gamut G-151}

\section{MULTIPLE HYPOINTENSE LIVER LESIONS ON T2-WEIGHTED MR IMAGES}

\section{COMMON}

1. Calcified granulomas

2. Regenerating nodules in cirrhosis

\section{UNCOMMON}

1. Gamna-Gandy bodies

2. Gas in biliary ducts or portal vein

3. Hydatid disease (Echinococcus granulosus or E. multilocularis with multiple calcified cysts)

4. Multifocal acute intrahepatic hemorrhages

5. Osler-Weber-Rendu disease

6. Periportal vascular collaterals

\section{Reference}

1. Gore RM: Liver: Differential diagnosis. In: Gore RM, Levine MS (eds): Textbook of Gastrointestinal Radiology. (ed 2) Philadelphia: WB Saunders, 2000, pp 1712-1727

2. Gore RM, Marn CS, Baron RL: Vascular disorders of the liver and splanchnic circulation. In: Gore RM, Levine MS (eds): Textbook of Gastrointestinal Radiology (ed 2) Philadelphia: WB Saunders, 2000, pp 1639-1668

3. Semelka RC, Kelekis NL: Liver. In: Semelka RC, Ascher SM, Reinhold C (eds): MRI of the Abdomen and Pelvis: A Text-Atlas. New York: Wiley-Liss, 1997, pp 19-136

\section{Gamut G-152}

\section{DIFFUSELY DECREASED LIVER INTENSITY ON MRI}

1. Hemochromatosis

2. Hemosiderosis

3. Superparamagnetic contrast medium

4. Wilson disease

\section{References}

1. Baron RL, Gore RM: Diffuse Liver Disease. In: Gore RM, Levine MS: Textbook of Gastrointestinal Radiology. (ed 2) Philadelphia: WB Saunders, 2000, pp 1590-1638

2. Gore RM: Liver: Differential diagnosis. In Gore RM, Levine MS (eds): Textbook of Gastrointestinal Radiology. (ed 2) Philadelphia: WB Saunders, 2000, pp 1712-1727

3. Mergo PJ, Ros PR: Benign lesions of the liver. Radiol Clin North Am 36:319-332, 1998

\section{Gamut G-153}

\section{SPONTANEOUS LIVER RUPTURE}

1. Hepatic tumor (eg, hepatocellular adenoma; hemangioma; hepatocellular carcinoma \{hepatoma\}

2. Oral contraceptive use

3. Oral estrogen therapy, long-term

4. Peliosis hepatis

5. Toxemia of pregnancy

\section{Reference}

1. Lundell CJ: Spontaneous hepatic rupture in postmenopausal women receiving oral estrogen replacement. JVIR 1993;4: 245-249 


\section{Gamut G-154}

\section{NEOPLASM OF THE LIVER (CHILD OR ADULT)}

\section{COMMON}

1. Hepatocellular adenoma

2. Cholangiocarcinoma, intrahepatic

3. [Cyst (eg, simple; posttraumatic; hydatid)]

4. Hemangioma (cavernous; capillary)

5. Hepatocellular carcinoma (hepatoma)

6. Metastasis

7. Multiple bile duct hamartoma (von Meyenburg complex) (microbiliary hamartoma)

8. [Nodular regenerative hyperplasia; focal nodular hyperplasia]

\section{UNCOMMON}

1. [Adrenal rest]

2. Biliary cystadenoma

3. Carcinoid of liver, primary or metastatic

4. Cholangioma

5. Epithelioid hemangioendothelioma

6. Fibrolamellar carcinoma

7. Gastrointestinal stromal tumor $_{\mathrm{g}}$ (eg, leiomyoma; fibroma)

8. Mesenchymal hamartoma (child)

9. Infantile hemangioendothelioma of liver (child)

10. Hepatoblastoma

11. Lymphangioma

12. Lymphoma

13. [Pancreatic rest]

14. Sarcoma (esp. angiosarcoma; also nonvascular sarcomas; Kaposi sarcoma)

15. [Splenosis]

16. Teratoma

[ ] This condition does not actually cause the gamuted imaging finding, but can produce imaging changes that simulate it.

\section{References}

1. Anthony CR: Tumors of the liver. Semin Roentgenol 1983;18:67-68
2. Buetow PC, Buck JL, Ros PR, et al: Malignant vascular tumors of the liver: radiologic-pathologic correlation. RadioGraphics 1994;14:153

3. Del Pilar Fernandez M, Reduanly RD: Primary hepatic malignant neoplasms. Radiol Clin North Am 1998;36:333-348

4. Kew MC: Hepatic tumors and cysts. In: Feldman M, Scharschmidt BF, Sleisenger MH: Gastrointestinal and Liver Disease. (ed 6) Philadelphia: WB Saunders, 2000

5. Paley MR, Ros PR; Hepatic metastases. Radiol Clin North Am 1998;36:349-364

6. Ros PR, Taylor HM: Malignant tumors of the liver. In: Gore RM, Levine MS (eds): Textbook of Gastrointestinal Radiology. (ed 2) Philadelphia: WB Saunders, 2000, pp 15231568

7. Sato M, Ishida H, Konno K, et al: Liver tumors in children and young patients: sonographic and color Doppler findings. Abdom Imaging 2000;25:596-601

8. Siegel MJ: Pediatric Body CT. Philadelphia: Lippincott Williams \& Wilkins, 1999

9. Stephens DH, Sheedy PF, Hattery RR, et al: Computed tomography of the liver. AJR 1977;128:579-590

10. Taybi H, Lachman RS: Radiology of Syndromes, Metabolic Disorders, and Skeletal Dysplasias. (ed 4) St. Louis: MosbyYear Book, 1996

11. Welch TJ, Sheedy PF, Johnson CM, et al: Radiographic characteristics of benign liver tumors: focal nodular hyperplasia and hepatic adenoma. RadioGraphics 1985;5:673

\section{Gamut G-155-1 \\ SOLID LIVER LESION-ADULT (US, CT, MRI)}

\section{COMMON}

1. Abscess (pyogenic, amebic, or fungal)

2. Focal fatty change (focal steatosis)

3. Focal nodular hyperplasia; adenomatous hyperplastic nodule

4. Hemangioma, cavernous or capillary

5. Hematoma

6. Hepatocellular adenoma

7. Hepatocellular carcinoma (hepatoma) - usually in cirrhotic or other damaged liver (eg, postradiation)

8. Metastasis (esp. from carcinoma of lung, breast, colon, kidney) 
9. Multiple bile duct hamartoma (von Meyenburg complex) (microbiliary hamartoma)

10. Regenerating or dysplastic nodule (cirrhosis)

\section{UNCOMMON}

1. Aneurysm of hepatic artery

2. Angiomyolipoma of liver

3. Angiosarcoma of liver

4. Bacillary angiomatosis (in AIDS)

5. Biliary cystadenoma, cystadenocarcinoma

6. Cholangiocarcinoma

7. Extramedullary hematopoiesis

8. Fibrolamellar carcinoma

9. Fungus disease (esp. histoplasmosis; candidiasis)

10. Hydatid disease (Echinococcus multilocularis or healed E. granulosus cyst)

11. Infarct

12. Kaposi sarcoma

13. Lipoma

14. Lymphoma

15. Tuberculosis; other granulomatous disease

16. Visceral larval migrans granuloma

\section{References}

1. Kehagias D, Moulopoulos L, Antoniou A, et al: Focal nodular hyperplasia. Eur Radiol 2001;11:202-212

2. Kim CK, Lim JH, Lee WJ: Detection of hepatocellular carcinomas and dysplastic nodules in cirrhotic liver. J Ultrasound Med 2001;20:99-104

3. Ros PR, Taylor HM: Benign and malignant tumors of the liver. In: Gore RM, Levine MS: Textbook of Gastrointestinal Radiology. (ed 2) Philadelphia: WB Saunders, 2000, pp 1487-1568

4. Ros PR, Taylor HM, Barreda R, et al: Focal hepatic infections. In: Gore RM, Levine MS: Textbook of Gastrointestinal Radiology. (ed 2) Philadelphia: WB Saunders, 2000, pp 1569-1589

5. Semelka RC, Kelekis NL: Liver. In: Semelka RC, Ascher SM, Reinhold C (eds): MRI of the Abdomen and Pelvis: A Text-Atlas. New York: Wiley-Liss, 1997, pp 19-136

6. Taylor HM, Ros PR: Hepatic imaging: an overview. Radiol Clin North Am 1998;36:237-245

7. Williamson MR: Abdominal ultrasound. In: Essentials of Ultrasound. Philadelphia: WB Saunders, 1996, p 86

\section{Gamut G-155-2}

\section{SOLID LIVER LESION-OLDER CHILD OR ADOLESCENT}

\section{COMMON}

1. Abscess (pyogenic, amebic, or fungal)

2. Hepatocellular carcinoma (hepatoma)

3. Metastasis (esp. neuroblastoma; sarcoma)

\section{UNCOMMON}

1. Angiomyolipoma

2. Fibrolamellar carcinoma

3. Focal nodular hyperplasia

4. Hemangioma

5. Hematoma

6. Hepatocellular adenoma (usually associated with glycogen storage disease, type I or Fanconi anemia)

7. Hydatid disease (Echinococcus multilocularis or healed E. granulosus cyst)

8. Infarct

9. Lipoma

10. Lymphoma ${ }_{\mathrm{g}}$; leukemia; Burkitt lymphoma

11. Mesenchymal hamartoma

12. Multiple bile duct hamartoma

13. Peliosis hepatis

14. Sarcoma of liver (eg, undifferentiated (embryonal); mixed mesenchymal); rhabdomyosarcoma of bile ducts

15. Teratocarcinoma

16. Tuberculosis; other granulomatous disease

\section{References}

1. Ros PR, Taylor HM: Benign and malignant tumors of the liver. In: Gore RM, Levine MS: Textbook of Gastrointestinal Radiology. (ed 2) Philadelphia: WB Saunders, 2000, pp 1487-1568

2. Ros PR, Taylor HM, Barreda R, et al: Focal hepatic infections. In: Gore RM, Levine MS: Textbook of Gastrointestinal Radiology. (ed 2) Philadelphia: WB Saunders, 2000, pp 1569-1589

3. Sato $\mathrm{M}$, Ishida $\mathrm{H}$, Konno $\mathrm{K}$, et al: Liver tumors in children and young patients: sonographic and color Doppler findings. Abdom Imaging 2000;25:596-601 
4. Siegel MJ: Pediatric Body CT. Philadelphia: Lippincott Williams \& Wilkins, 1999

5. Taylor HM, Ros PR: Hepatic imaging: an overview. Radiol Clin North Am 1998;36:237-245

\section{Gamut G-155-3}

\section{SOLID LIVER LESION-INFANT OR YOUNG CHILD (Under Age 5)}

\section{COMMON}

*1. Hemangioma

2. Hepatoblastoma

*3. Infantile hemangioendothelioma

\section{UNCOMMON}

1. Abscess

2. Hematoma

3. Mesenchymal hamartoma

4. Metastasis (esp. neuroblastoma; sarcoma)

* Especially newborn to 6 months.

\section{References}

1. Cohen MD: Imaging of Children with Cancer. St. Louis: Mosby-Year Book, 1992, pp 20-42

2. Ros PR, Taylor HM: Benign and malignant tumors of the liver. In: Gore RM, Levine MS: Textbook of Gastrointestinal Radiology. (ed 2) Philadelphia: WB Saunders, 2000, pp 1487-1568

3. Sato M, Ishida H, Konno K, et al: Liver tumors in children and young patients: sonographic and color Doppler findings. Abdom Imaging 2000;25:596-601

4. Siegel MJ: Pediatric Body CT. Philadelphia: Lippincott Williams \& Wilkins, 1999

\section{Gamut G-156}

\section{SOLID LIVER MASSES-MULTIPLE}

\section{COMMON}

1. Abscess (pyogenic, amebic, or fungal)

2. Fatty change in liver (steatosis)

3. Focal nodular hyperplasia

4. Fungus disease (eg, candidiasis; histoplasmosis)

5. Granulomatous disease (eg, sarcoidosis; tuberculosis)

6. Hemangioma

7. Hematoma (intrahepatic or subcapsular)

8. Lymphoma $_{\mathrm{g}}$; Burkitt lymphoma

9. Metastases (esp. from carcinoma of lung, breast, colon, kidney)

10. Regenerating or dysplastic nodules (cirrhosis)

\section{UNCOMMON}

1. Angiosarcoma of liver

2. Bacillary angiomatosis (in AIDS)

3. Cholangiocarcinoma

4. Cytomegalovirus infection, multifocal

5. Drug-induced toxicity (eg, erythromycin; diphenylhydantoin)

6. Extramedullary hematopoiesis

7. Hepatic cystadenoma

8. Hepatocellular adenoma; adenomatosis

9. Hepatocellular carcinoma (hepatoma)

10. Hydatid disease (Echinococcus multilocularis or healed, calcified E. granulosus cysts)

11. Kaposi sarcoma

12. Mesenchymal hamartoma

\section{References}

1. Gore RM: Liver: Differential diagnosis. In: Gore RM, Levine MS: Textbook of Gastrointestinal Radiology. (ed 2) Philadelphia: WB Saunders, 2000, pp 1712-1727

2. Ros PR, Taylor HM: Benign and malignant tumors of the liver. In: Gore RM, Levine MS: Textbook of Gastrointestinal Radiology. (ed 2) Philadelphia: WB Saunders, 2000, pp 1487-1568

3. Ros PR, Taylor HM, Barreda R, et al: Focal hepatic infections. In: Gore RM, Levine MS: Textbook of Gastrointesti- 
nal Radiology. (ed 2) Philadelphia: WB Saunders, 2000, pp 1569-1589

4. Sato M, Ishida H, Konno K, et al: Liver tumors in children and young patients: sonographic and color Doppler findings. Abdom Imaging 2000;25:596-601

5. Semelka RC, Kelekis NL: Liver. In: Semelka RC, Ascher SM, Reinhold C (eds): MRI of the Abdomen and Pelvis: A Text-Atlas. New York: Wiley-Liss, 1997, pp 19-136

6. Williamson MR: Abdominal ultrasound. In: Essentials of Ultrasound. Philadelphia: WB Saunders, 1996, p 86

7. Withers CE, Wilson SR: The liver. In: Rumack CM, Wilson SR, Charboneau JW (eds): Diagnostic Ultrasound. (ed 2) St. Louis: Mosby, 1998, pp 87-154

\section{Gamut G-157}

\section{CYSTIC LIVER LESION(S) (US, CT, MRI) (Usually Anechoic or Hypoechoic on US and Low Density on CT)}

\section{COMMON}

1. Abscess (pyogenic, amebic, or fungal)

2. Cyst, congenital or acquired (eg, epithelial; posttraumatic; hydatid)

3. Cystic metastasis (eg, mucinous adenocarcinoma of colon, stomach; cystadenocarcinoma of pancreas, ovary, or uterus; melanoma, carcinoid, or sarcoma with necrosis)

4. [Chilaiditi disease (interposition of colon between liver and diaphragm]

5. Hematoma, acute

6. Hydatid disease (Echinococcus granulosus and E. multilocularis)

\section{UNCOMMON}

1. Aneurysm of hepatic artery or portal vein

2. Biliary cystadenoma, cystadenocarcinoma

3. Biloma

4. Caroli disease

5. Cat-scratch disease

6. Cholangiocarcinoma
7. Choledochal cyst

8. Cystic duct remnant with mucocele

9. Cystic hepatocellular carcinoma (hepatoma)

10. Cystic lymphangioma, mesenchymal hamartoma, or other unusual cystic or necrotic tumor

11. Intrahepatic gallbladder

12. Mesenchmal hamartoma

13. Multiple bile duct hamartoma (Von Meyenburg complex)

14. Polycystic liver disease

15. Undifferentiated (embryonal) sarcoma

16. Von Hippel-Lindau disease

[ ] This condition does not actually cause the gamuted imaging finding, but can produce imaging changes that simulate it.

\section{References}

1. Eisenberg RL: Clinical Imaging: An Atlas of Differential Diagnosis. (ed 3) Philadelphia: Lippincott-Raven, 1997, pp 484-487

2. Ferrozzi F, Bova D, Campodonico F: Cystic primary neoplasms of the liver of the adult: CT features. Clin Imag 1993; 17:292-296

3. Luo T, Itai Y, Eguchi N, et al: Von Meyenburg complexes of the liver: imaging findings. J Comput Assist Tomogr 1998; 22:372-378

4. Mergo PJ, Ros PR: Benign lesions of the liver. Radiol Clin North Am 1998;36:365-376

5. Parulekar SG, Bree RL: Liver. In: McGahan JP, Goldberg BB (eds): Diagnostic Ultrasound. Philadelphia: LippincottRaven, 1997, chapter 21

6. Semelka RC, Kelekis NL: Liver. In: Semelka RC, Ascher SM, Reinhold C (eds): MRI of the Abdomen and Pelvis: A Text-Atlas. New York: Wiley-Liss, 1997, pp 19-136

7. Singh Y, Winick AB, Tabbara SO: Multiloculated cystic liver lesions: radiologic-pathologic differential diagnosis. RadioGraphics 1997;17:219-224

8. Skolnick ML: Guide to the Ultrasound Examination of the Abdomen. New York: Springer-Verlag, 1986, pp 89-90

9. Withers CE, Wilson SR: The liver. In: Rumack CM, Wilson SR, Charboneau JW (eds): Diagnostic Ultrasound. (ed 2) St. Louis: Mosby, 1998, pp 87-154 


\section{Gamut G-158-1}

\section{LIVER LESION CHARACTERIZED BY LINEAR OR STELLATE CENTRAL SCAR (CT, US, ANGIO)}

1. Cholangiocarcinoma

2. Fibrolamellar carcinoma

*3. Focal nodular hyperplasia

4. Hemangioma, giant cavernous

5. Hepatocellular adenoma

*6. Hepatocellular carcinoma (hepatoma)

7. Metastasis, hypervascular

* Usually isoechoic mass with hyperechoic linear or stellate central scar on ultrasound.

\section{References}

1. Gore RM: Liver: Differential diagnosis. In: Gore RM, Levine MS: Textbook of Gastrointestinal Radiology. (ed 2) Philadelphia: WB Saunders, 2000, pp 1712-1727

2. Parulekar SG, Bree RL: Liver. In: McGahan JP, Goldberg BB (eds): Diagnostic Ultrasound. Philadelphia: LippincottRaven, 1997, chapter 21

3. Ros PR, Taylor HM: Benign and malignant tumors of the liver. In: Gore RM, Levine MS: Textbook of Gastrointestinal Radiology. (ed 2) Philadelphia: WB Saunders, 2000, pp 1487-1568

4. Withers CW, Wilson SR: The liver. In: Rumack CM, Wilson SR, Charboneau JW (eds): Diagnostic Ultrasound. St. Louis: Mosby, 1998, pp 87-154

\section{Gamut G-158-2}

\section{LIVER LESION WITH CENTRAL SCAR ON MRI}

1. Cavernous hemangioma: hypo- or hyperintense $\mathrm{T} 2$-weighted image (either inflammatory or fibrous scar)

2. Fibrolamellar hepatocellular carcinoma: hypointense T1-WI, hyperintense T2-WI (fibroticrepair of scar)
3. Focal nodular hyperplasia: hypointense T1-WI, hyperintense T2-WI (inflammatory scar)

4. Hepatocellular adenoma: variable signal

\section{References}

1. Pomeranz SJ; Gamuts and Pearls in MRI. (ed 2) Cincinnati: MRI-EFI Publications, 1993

2. Ros PR, Taylor HM: Benign and malignant tumors of the liver. In: Gore RM, Levine MS: Textbook of Gastrointestinal Radiology. (ed 2) Philadelphia: WB Saunders, 2000, pp 1487-1568

3. Semelka RC, Kelekis NL: Liver. In: Semelka RC, Ascher SM, Reinhold C (eds): MRI of the Abdomen and Pelvis: A Text-Atlas. New York: Wiley-Liss, 1997, pp 19-136

\section{Gamut G-159}

\section{LIVER LESION CHARACTERIZED BY BLOOD OR HEMORRHAGE}

\section{COMMON}

1. Hemangioma

2. Hematoma

3. Hepatocellular adenoma

4. Hepatocellular carcinoma (hepatoma)

\section{UNCOMMMON}

1. Angiosarcoma of liver

2. Bacillary angiomatosis (in AIDS)

3. Kaposi sarcoma

4. Peliosis hepatis

\section{References}

1. Mergo PJ, Ros PR: Benign lesions of the liver. Radiol Clin North Am 1998;36:365-376

2. Ros PR, Taylor HM: Benign and malignant tumors of the liver. In: Gore RM, Levine MS: Textbook of Gastrointestinal Radiology. (ed 2) Philadelphia: WB Saunders, 2000, pp 1487-1568 


\section{Gamut G-160}

\section{LIVER LESION CHARACTERIZED BY CALCIFICATION}

\section{COMMON}

1. Abscess, healed (pyogenic, amebic, or fungal)

2. Calculus(i) in biliary tract

3. Granuloma (eg, tuberculosis; histoplasmosis; coccidioidomycosis; brucellosis)

4. Hemangioma, cavernous

5. Hematoma, old

6. Hepatocellular carcinoma (hepatoma), esp. treated

7. Hydatid disease (Echinococcus granulosus or E. multilocularis)

8. Metastases (eg, from osteosarcoma; chondrosarcoma; mucinous or colloid adenocarcinoma of colon, rectum, ovary, breast, pancreas, stomach, or thyroid; islet cell carcinoma; carcinoid; teratocarcinoma; leiomyosarcoma; neuroblastoma; pheochromocytoma; treated melanoma or lymphoma)

\section{UNCOMMON}

1. Biliary cystadenocarcinoma

2. Calcified gallbladder (porcelain gallbladder)

3. Calcified hepatic artery (incl. aneurysm)

4. Cholangiocarcinoma

5. Cyst, nonparasitic, congenital or acquired

6. Fibrolamellar carcinoma

7. Gumma (hepar lobatum)

8. Hemangioendothelioma (epithelioid or infantile)

9. Hepatoblastoma

10. Hepatocellular adenoma

11. Mesenchymal hamartoma

12. Regenerating nodules in cirrhosis (rarely)
13. Schistosomiasis (turtleshell appearance, esp. $S$. japonica)

14. Thromboembolus of portal vein, calcified

\section{References}

1. Darlak JJ, Moskowitz M, Katten KR: Calcifications in the liver. Radiol Clin North Am 1980;18:209-219

2. Paley MR, Ros PR: Hepatic calcification. Radiol Clin North Am 1998;36:391-398

3. Parulekar SG, Bree RL: Liver. In: McGahan JP, Goldberg BB: Diagnostic Ultrasound. Philadelphia: LippincottRaven, 1997, chapter 21

4. Stoupis C, Taylor HM, Paley MR, et al: The rocky liver: radiologic-pathologic correlation of calcified hepatic masses. Radiographics 1998;18:675-685

\section{Gamut G-161}

\section{LIVER LESION CHARACTERIZED BY FAT (CT, MRI)}

1. Angiomyolipoma of liver

2. Focal fatty change

3. Focal nodular hyperplasia

4. Hepatocellular adenoma

5. Hepatocellular carcinoma (hepatoma)

6. Langerhans cell histiocytosis ${ }_{\mathrm{g}}$

7. Lipoma

8. Metastasis (eg, liposarcoma; teratoid tumor)

9. Myelolipoma

\section{References}

1. Roberts JL, Fishman EK, Hartman DS, et al.: Lipomatous tumors of the liver: evaluation with CT and US. Radiology 1986;158:613-617

2. Ros PR: Hepatic angiomyolipoma: is fat in the liver friend or foe? Abdom Imaging 1994;19:552-553 


\section{Gamut G-162}

\section{LIVER LESION CHARACTERIZED BY BULL'S-EYE APPEARANCE}

1. Fungus disease (eg, candidiasis, usually in immunocompromised individual); other opportunistic infections

2. Kaposi sarcoma

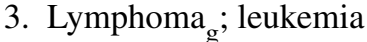

4. Metastasis

5. Sarcoidosis

6. Septic emboli

\section{References}

1. Dähnert W: Radiology Review Manual. (ed 4) Baltimore, Williams \& Wilkins, 1999

2. Paley MR, Ros PR: Hepatic metastases. Radiol Clin North Am 1998;36:349-364

\section{Gamut G-163}

\section{LIVER LESION CHARACTERIZED BY FLUID-FLUID LEVEL}

1. Biliary cystadenoma

2. Hemangioma

3. Hematoma

4. Hepatic cyst (with hemorrhage or infection)

5. Hepatocellular adenoma (hemorrhagic)

6. Hepatocellular carcinoma (hepatoma), cystic

7. Metastasis (eg, leiomyosarcoma; adenocarcinoma of lung or ovary; carcinoid)

\section{Reference}

1. Soyer P, Bluemke DA, Fishman EK, Rymer R: Fluid-fluid levels within focal hepatic lesions: imaging appearance and etiology. Abd Imag 1998;23:161-165

\section{Gamut G-164}

LIVER LESION CHARACTERIZED BY "FILL-IN"

\section{(ANGIO, POSTCONTRAST CT OR MRI)}

1. Cholangiocarcinoma

2. Focal nodular hyperplasia

3. Hemangioendothelioma (epithelioid or infantile)

4. Hemangioma

5. Hepatocellular carcinoma (hepatoma)

6. Kaposi sarcoma

7. Leiomyosarcoma

8. Lymphoma

9. Metastasis

10. Vascular malformation

\section{References}

1. Ito K, Honjo K, Fujita T, et al: Liver neoplasms: diagnostic pitfalls in cross sectional imaging. RadioGraphics 1996; $16: 273-283$

2. Shirkhoda A, Salmanzadeh A: Hepatic lesions which "fillin" on contrast-enhanced CT and MR imaging: patterns and diagnostic pitfalls

\section{Gamut G-165-S}

\section{LIVER LESIONS-CLINICAL CONSIDERATIONS}

\section{Nonsurgical Liver Lesions}

1. Abscess (except for possible drainage)

2. Cyst (eg, simple; posttraumatic; hydatid)

3. Fatty liver

4. Focal nodular hyperplasia

5. Hemangioma

\section{Painless Liver Lesions}

1. Cyst (eg, simple; hydatid)

2. Focal nodular hyperplasia

3. Hemangioma

4. Hepatocellular adenoma (small) 


\section{Liver Lesions Related to Excessive Steroids}

1. Focal nodular hyperplasia

2. Hemangioma

3. Hepatocellular adenoma

4. Hepatocellular carcinoma (hepatoma)

5. Nodular regenerative hyperplasia

\section{Liver Neoplasms With Elevated Alpha Fetal Protein (AFP)}

1. Hepatoblastoma

2. Hepatocellular carcinoma (hepatoma)

\section{Sex Predilection for Liver Neoplasms}

1. Hepatic neoplasm is usually benign in women

2. Hepatic neoplasm is usually malignant in men

\section{Reference}

1. Ros PR, Taylor HM: Benign and malignant tumors of the liver. In: Gore RM, Levine MS: Textbook of Gastrointestinal Radiology. (ed 2) Philadelphia: WB Saunders, 2000, pp 1487-1568

\section{Gamut G-166}

\section{LIVER METASTASES-CALCIFIED}

\section{COMMON}

1. Endocrine carcinoma of pancreas (esp. islet cell tumor)

2. Malignant melanoma

3. Medullary or colloid carcinoma of breast or thyroid

4. Mucinous adenocarcinoma of colon or stomach

5. Neuroblastoma

6. Papillary serous cystadenocarcinoma of ovary

7. Sarcoma (esp. osteosarcoma; chondrosarcoma; leiomyosarcoma)

\section{UNCOMMON}

1. Carcinoma of lung or testis

2. Lymphoma ${ }_{\mathrm{g}}$, treated
3. Mesothelioma

4. Renal cell carcinoma

\section{References}

1. Burgener FA, Kormano M: Differential Diagnosis in Computed Tomography. New York: Thieme, 1996

2. Dähnert W: Radiology Review Manual. (ed 4) Baltimore: Williams \& Wilkins, 1999, p 596

3. Paley MR, Ros PR: Hepatic metastases. Radiol Clin North Am 1998;36:349-364

4. Ros PR, Taylor HM: Malignant tumors of the liver. In: Gore RM, Levine MS (eds): Textbook of Gastrointestinal Radiology. (ed 2) Philadelphia: WB Saunders, 2000, pp 1523-1568

\section{Gamut G-167 \\ LIVER METASTASES- HYPERVASCULAR}

\section{COMMON}

1. Carcinoid

2. Carcinoma of breast

3. Carcinoma of colon

4. Choriocarcinoma

5. Endocrine carcinoma of pancreas (esp. islet cell tumor)

6. Malignant melanoma

7. Renal cell carcinoma (hypernephroma)

\section{UNCOMMON}

1. Cystadenocarcinoma of ovary

2. Pheochromocytoma

3. Sarcoma (esp. osteosarcoma; chondrosarcoma; leiomyosarcoma)

\section{References}

1. Dähnert W: Radiology Review Manual. (ed 4) Baltimore: Williams \& Wilkins, 1999, p 596

2. Paley MR, Ros PR: Hepatic metastases. Radiol Clin North Am 1998;36:349-364

3. Ros PR, Taylor HM: Malignant tumors of the liver. In: Gore RM, Levine MS (eds): Textbook of Gastrointestinal Radiology. (ed 2) Philadelphia: WB Saunders, 2000, pp 15231568 


\section{Gamut G-168}

\section{LIVER METASTASES-HEMORRHAGIC}

\section{COMMON}

1. Carcinoma of breast

2. Carcinoma of colon

3. Carcinoma of thyroid

4. Choriocarcinoma

5. Malignant melanoma

6. Renal cell carcinoma (hypernephroma)

\section{References}

1. Dähnert W: Radiology Review Manual. (ed 4) Baltimore: Williams \& Wilkins, 1999, p 597

2. Paley MR, Ros PR: Hepatic metastases. Radiol Clin North Am 1998;36:349-364

3. Ros PR, Taylor HM: Malignant tumors of the liver. In: Gore RM, Levine MS (eds): Textbook of Gastrointestinal Radiology. (ed 2) Philadelphia: WB Saunders, 2000, pp 15231568

\section{Gamut G-169}

\section{LIVER METASTASES-CYSTIC}

\section{COMMON}

1. Carcinoma of colon

2. Choriocarcinoma

3. Malignant melanoma

4. Mucinous carcinoma of ovary

5. Sarcoma (eg, osteosarcoma; chondrosarcoma; leiomyosarcoma)

\section{UNCOMMON}

1. Carcinoid

2. Carcinoma of lung (esp. small cell)

3. Carcinoma of stomach

4. Endometrial carcinoma of uterus

\section{Reference}

1. Dähnert W: Radiology Review Manual. (ed 4) Baltimore: Williams \& Wilkins, 1999, p 597

\section{Gamut G-170 \\ LIVER METASTASES- \\ ULTRASOUND CHARACTERISTICS}

\author{
HYPOECHOIC LESIONS (37.5\%) \\ COMMON \\ 1. Carcinoma of breast \\ 2. Carcinoma of pancreas $(36 \%)$ \\ 3. Carcinoma of cervix $(20 \%)$ \\ 4. Lymphoma ${ }_{\mathrm{g}}$
}

\section{UNCOMMON}

1. Adenocarcinoma of lung

2. Carcinoma of nasopharynx

\section{MIXED ECHOGENICITY (37.5\%)}

\section{COMMON}

1. Carcinoma of breast

2. Carcinoma of rectum

3. Carcinoma of lung

4. Carcinoma of stomach

5. Hepatocellular carcinoma (hepatoma)

\section{UNCOMMON}

1. Anaplastic carcinoma

2. Carcinoma of cervix

3. Vascular primaries (carcinoid; islet cell carcinoma of pancreas; choriocarcinoma; renal cell carcinoma)

\section{HYPERECHOIC LESIONS WITH SHADOWING (25\%)}

\section{COMMON}

1. Adenocarcinoma of stomach

2. Cystadenocarcinoma of pancreas

3. Melanoma

4. Mucinous or colloid carcinoma of colon or breast

5. Pseudomucinous cystadenocarcinoma of ovary 


\section{UNCOMMON}

1. Chondrosarcoma

2. Neuroblastoma

3. Osteosarcoma

4. Teratocarcinoma

\section{CYSTIC METASTASES (See G-169)}

1. Central necrosis of any malignant lesion, esp. sarcomas

2. Mucin-secreting metastases from carcinoma of the ovary, colon, pancreas, or stomach

\section{BULL'S-EYE OR TARGET PATTERN}

1. Carcinoma of lung

2. Other carcinomas (occasionally)

\section{INFILTRATIVE PATTERN}

1. Carcinoma of breast

2. Carcinoma of lung

3. Melanoma

\section{References}

1. Dähnert W: Radiology Review Manual. (ed 4) Baltimore: Williams \& Wilkins, 1999, p 597

2. Gore RM: Liver: Differential diagnosis. In Gore RM, Levine MS (eds): Textbook of Gastrointestinal Radiology. (ed 2) Philadelphia: WB Saunders, 2000, pp 1712-1727

3. Paley MR, Ros PR: Hepatic metastases. Radiol Clin North Am 1998;36:349-364

\section{Gamut G-171}

\section{AVASCULAR OR HYPOVASCULAR LIVER LESION (ANGIO, POSTCONTRAST CT OR MRI)}

\section{COMMON}

*1. Abscess (eg, pyogenic, amebic, or fungal)

2. Cholangiocarcinoma

*3. Cyst (congenital; inflammatory; hydatid; traumatic)

4. [Extrinsic mass (eg, gallbladder; adrenal neoplasm; subphrenic abscess)]

*5. Focal fatty change

*6. Metastasis

\section{UNCOMMON}

1. Adrenal rest

2. [Biloma, traumatic or iatrogenic (eg, needle biopsy)]

3. Hematoma

*4. Lymphoma ${ }_{\mathrm{g}}$

5. Mesenchymal hamartoma

6. Neoplasm, benign or malignant, other (eg, gastrointestinal stromal tumor ${ }_{\mathrm{g}}$; teratoma; lipoma)

*7. Polycystic disease of liver

* May be multiple.

[ ] This condition does not actually cause the gamuted imaging finding, but can produce imaging changes that simulate it.

\section{References}

1. Guermazi A, Brice P, de Kerviler E, et al: Extranodal Hodgkin disease: spectrum of disease. RadioGraphics 2001; 21:161-179

2. Ros PR, Taylor HM: Benign and malignant tumors of the liver. In: Gore RM, Levine MS: Textbook of Gastrointestinal Radiology. (ed 2) Philadelphia: WB Saunders, 2000, pp 1487-1568 


\section{Gamut G-172 \\ HYPERVASCULAR OR HYPERDENSE LIVER LESION (ANGIO, POSTCONTRAST CT OR MRI)}

\section{COMMON}

*1. Focal fatty sparing (simulates enhancing mass)

*2. Focal nodular hyperplasia

*3. Hemangioma, cavernous or capillary

4. Hepatoblastoma

5. Hepatocellular adenoma

6. Hepatocellular carcinoma (hepatoma)

*7. Metastasis, hypervascular (eg, carcinoid; islet cell tumor of pancreas; pheochromocytoma; leiomyosarcoma; other sarcomas; choriocarcinoma; carcinoma of breast, colon, kidney, or thyroid; malignant melanoma)

\section{UNCOMMON}

1. Aneurysm of hepatic artery, true or false (incl. arteriobiliary fistula) or portal vein

2. Angiosarcoma of liver

*3. Arteriovenous fistula (eg, congenital; traumatic; iatrogenic); arterio-portal shunt in some hepatomas

4. Fibrolamellar carcinoma

5. Hemangioendothelioma, epithelioid

6. Vascular phenomena (eg, SVC obstruction; BuddChiari S.; transient hepatic attenuation difference $\{$ THAD $\}$ )

* May be multiple.

\section{References}

1. Adler J, Goodgold M, Mitty H, et al: Arteriovenous shunts involving the liver. Radiology 1978;129:315-322

2. Bressler EL, Alpern MB, Glazer GM, et al: Hypervascular hepatic metastases: CT evaluation. Radiology 1987;162; $49-51$

3. Kehagias D, Moulopoulos L, Antoniou A, et al: Focal nodular hyperplasia: imaging findings. Eur Radiol 2001;11: 202-212

4. Murakami T, Kim T, Takamura M, et al: Hypervascular hepatocellular carcinoma: detection with double arterial phase multi-detector helical CT. Radiology 2001;218:763-767
5. Oliver JH, Baron RL, Federle MP, et al: Hypervascular liver metastasis: do unenhanced and hepatic arterial phase CT images affect tumor detection? Radiology 1997;205: 709-715

6. Quiroga S, Sebastia C, Pallisa E, et al: Improved diagnosis of hepatic perfusion disorders: Value of hepatic arterial phase imaging during helical CT. RadioGraphics 2001; $21: 65-81$

7. Slone RM, Fisher AJ: Pocket Guide to Body CT Differential Diagnosis. New York: McGraw-Hill, 1999, pp 219-220

\section{Gamut G-173}

\section{ANECHOIC LIVER LESION (USUALLY CYSTIC ON US)}

\section{COMMON}

*1. Caroli disease

*2. Congenital simple cyst

3. Metastasis, cystic (eg, from mucinous carcinoma of colon; carcinoma of stomach or uterus; cystadenocarcinoma of ovary or pancreas; squamous cell carcinoma, esp. bronchogenic; carcinoid; malignant melanoma; sarcoma, esp. leiomyosarcoma)

*4. Polycystic liver disease

\section{UNCOMMON}

1. Abscess (pyogenic, amebic, or fungal)

*2. Acquired cyst (eg, posttraumatic; inflammatory)

*3. Aneurysm of hepatic artery or portal vein

4. Biliary cystadenoma, cystadenocarcinoma

*5. Biloma

*6. [Chilaiditi disease (interposition of colon between liver and diaphragm)]

*7. Choledochal cyst

8. Cystic or necrotic neoplasm

*9. Cystic duct remnant with mucocele

10. Hematoma, acute

11. Hydatid cyst

*12. Intrahepatic gallbladder

13. Lymphoma ${ }_{g}$

* Usually unilocular with smooth walls and distal acoustic enhancement. [ ] This condition does not actually cause the gamuted imaging finding, but can produce imaging changes that simulate it. 


\section{References}

1. Eisenberg RL: Clinical Imaging: An Atlas of Differential Diagnosis. (ed 3) Philadelphia: Lippincott-Raven, 1997, pp 484-487

2. Parulekar SG, Bree RL: Liver. In: McGahan JP, Goldberg BB (eds): Diagnostic Ultrasound. Philadelphia: LippincottRaven, 1997, chapter 21

3. Skolnick ML: Guide to the Ultrasound Examination of the Abdomen. New York: Springer-Verlag, 1986, pp 89-90

4. Williamson MR: Abdominal ultrasound. In: Essentials of Ultrasound. Philadelphia: WB Saunders, 1996, p 81

5. Withers CE, Wilson SR: The liver. In: Rumack CM, Wilson SR, Charboneau JW (eds): Diagnostic Ultrasound. (ed 2) St. Louis: Mosby, 1998, pp 87-154

\section{Gamut G-174}

\section{HYPOECHOIC LIVER LESION (US)}

\section{COMMON}

1. Abscess (pyogenic, amebic, or fungal)

2. Cyst, complicated (eg, with cholesterol crystals)

*3. Cystic liver tumor (eg, cholangiocarcinoma; biliary cystadenoma or cystadenocarcinoma; lymphangioma; mesenchymal hamartoma)

+4. Focal nodular hyperplasia

5. Hematoma, acute

+6. Hepatocellular carcinoma (hepatoma)

7. Infarct

8. Lymphoma ; $_{\mathrm{g}}$ Burkitt lymphoma

+9. Metastasis, incl. cystic metastasis (eg, from carcinoma of breast, lung, colon, stomach, ovary, pancreas)

\section{UNCOMMON}

1. Biloma

+2. Candidiasis (wheel within a wheel)

3. Extramedullary hematopoiesis

4. Focal hepatic necrosis

5. Focal sparing in fatty liver (steatosis)

6. Granuloma (eg, tuberculosis)

7. Hemangioendothelioma of liver

8. Hemangioma, cavernous (more often hyperechoic)
+9. Hepatocellular adenoma

*10. Hydatid disease (Echinococcus granulosus or E. multilocularis)

* Usually a multicystic image on ultrasound.

+ Hepatic mass with hypoechoic halo.

\section{References}

1. Gore RM: Liver: differential diagnosis. In: Gore RM, Levine MS: Textbook of Gastrointestinal Radiology. (ed 2) Philadelphia: WB Saunders, 2000, pp 1712-1726

2. Parulekar SG, Bree RL: Liver. In: McGahan JP, Goldberg BB (eds): Diagnostic Ultrasound. Philadelphia: LippincottRaven, 1997, chapter 21

3. Skolnick ML: Guide to the Ultrasound Examination of the Abdomen. New York: Springer-Verlag, 1986, pp 91-92

4. Weill FS: Ultrasound Diagnosis of Digestive Diseases. (ed 3 revised) Berlin: Springer-Verlag, 1990, pp 239-246

5. Withers CE, Wilson SR: The liver. In: Rumack CM, Wilson SR, Charboneau JW (eds): Diagnostic Ultrasound. St. Louis: Mosby-Year Book, 1998, pp 87-154

\section{Gamut G-175}

\section{HYPERECHOIC LIVER LESION (US)}

\section{COMMON}

1. Cirrhosis (multifocal regenerating nodules)

2. Focal fatty infiltration (focal steatosis)

*3. Hemangioma

4. Hepatocellular adenoma

*5. Hepatocellular carcinoma (hepatoma)

6. Lipoma

7. Metastasis \{esp. from carcinoma of colon, stomach, ovary, kidney or pancreas \{incl. islet cell carcinoma \}; carcinoid*; choriocarcinoma)

\section{UNCOMMON}

1. Angiomyolipoma

2. Debris inside abscess or hematoma

3. Dysplastic nodule

4. Focal nodular hyperplasia

*5. Hemangioendothelioma

6. Hepatic fissure

(continued) 
7. Hydatid disease (old healed, calcified E. granulosus cyst; Echinococcus multilocularis-multifocal)

8. Infection (eg, cytomegalovirus or Candida)

9. Lymphoma

10. Omentum inserted into bed of hepatic resection

*11. Peliosis hepatis

12. Postradiation therapy

13. Solitary fibrous tumor of the liver

* Hyperechoic hepatic mass(es) with punctate calcifications and/or acoustic enhancement on ultrasound.

\section{References}

1. Fuksbrumer MS, Klimstra D, Panicek DM: Solitary fibrous tumor of the liver: Imaging findings. AJR 2000;175:16831687

2. Numata K, Tanaka K, Kiba T, et al: Contrast-enhanced, wide-band harmonic gray scale imaging of hepatocellular carcinoma. J Ultrasound Med 2001;20:89-98

3. Parulekar SG, Bree RL: Liver. In: McGahan JP, Goldberg BB (eds): Diagnostic Ultrasound. Philadelphia: LippincottRaven, 1997, chapter 21

4. Ros PR, Taylor HM: Benign and malignant tumors of the liver. In: Gore RM, Levine MS: Textbook of Gastrointestinal Radiology. (ed 2) Philadelphia: WB Saunders, 2000, pp 1487-1568

5. Rumack CM, Wilson SR, Charboneau JW (eds): Diagnostic Ultrasound. St. Louis: Mosby, 1998

6. Weill FS: Ultrasound Diagnosis of Digestive Diseases. (ed 3 revised) Berlin: Springer-Verlag, 1990, pp 239-246

7. Withers CW, Wilson SR: The liver. In: Rumack CM, Wilson SR, Charboneau JW (eds): Diagnostic Ultrasound. St. Louis: Mosby-Year Book, 1998, pp 87-154

\section{Gamut G-176}

\section{ISOECHOIC LIVER LESION (US)}

1. Focal nodular hyperplasia

2. Hepatocellular adenoma

3. Hepatocellular carcinoma (hepatoma)

\section{Reference}

1. Withers CW, Wilson SR: The liver. In: Rumack CM, Wilson SR, Charboneau JW (eds): Diagnostic Ultrasound. St. Louis: Mosby-Year Book, 1998, pp 87-154
Gamut G-177

\section{HETEROGENEOUS HEPATIC ECHOGENICITY ON ULTRASOUND (COMPLEX HEPATIC MASS OR DIFFUSE PARENCHYMAL INVOLVEMENT)}

\section{COMMON}

1. Abscess

2. Cavernous hemangioma

*3. Cirrhosis

*4. Fatty change in liver

5. Focal nodular hyperplasia

6. Hepatocellular carcinoma (hepatoma)

7. Hydatid cyst (with collapsed daughter cysts)

*8. Metastasis with mixed echogenicity or diffuse infiltration (eg, from carcinoma of breast, lung, or ovary; melanoma)

9. Neoplasm with liquefaction necrosis (hypoechoic) and infarcted nonliquefied areas (hyperechoic)

\section{UNCOMMON}

1. Biliary cystadenoma

2. Cholangiocarcinoma

3. Hemangioendothelioma; angiosarcoma

4. Hepatic adenoma, esp. after oral contraceptive use, or in glycogen storage disease, type I (von Gierke disease)

5. Hepatoblastoma

*6. Peliosis hepatitis

* Usually generalized or multifocal.

\section{References}

1. Eisenberg RL: Clinical Imaging: An Atlas of Differential Diagnosis. Gaithersburg, MD: Aspen Publ, 1992

2. Parulekar SG, Bree RL: Liver. In: McGahan JP, Goldberg BB (eds): Diagnostic Ultrasound. Philadelphia: LippincottRaven, 1997, chapter 21

3. Skolnick ML: Guide to the Ultrasound Examination of the Abdomen. New York: Springer-Verlag, 1986, p 93

4. Withers CW, Wilson SR: The liver. In: Rumack CM, Wilson SR, Charboneau JW (eds): Diagnostic Ultrasound. St. Louis: Mosby, 1998, pp 87-154 


\section{Gamut G178-S}

\section{HEPATIC PSEUDOLESION (US)}

1. Diaphragmatic leaflets: peripheral echogenic pseudolesion may simulate hemangiomas

2. Falciform ligament: echogenic "mass" (pseudolesion) in left lobe

3. Focal fatty infiltration: echogenic pseudolesion may simulate metastases

4. Focal hepatic sparing in steatosis: hypoechoic pseudolesion often seen in porta hepatis

5. Gallbladder inflammation: hypoechoic hepatic pseudolesion in adjacent parenchyma

6. Ligamentum venosum: fibrous tissue attenuates sound, causing hypoechoic pseudolesion in caudate lobe

7. Perihepatic fat may invaginate liver causing hyperechoic masses

\section{Reference}

1. Gore RM: Liver: Differential diagnosis. In Gore RM, Levine MS (eds): Textbook of Gastrointestinal Radiology. (ed 2) Philadelphia: WB Saunders, 2000, pp 1712-1727

\section{Gamut G-179}

\section{INTRAHEPATIC ACOUSTIC SHADOWING (LINEAR VERSUS FOCAL) ON US}

\section{LINEAR OR BRANCHING SHADOWING}

1. Air in biliary tract

2. Air in portal vein

3. Calculi in biliary ducts

\section{FOCAL SHADOWING}

1. Calcification (eg, primary or metastatic tumor; granuloma; healed abscess; aneurysm)

2. Foreign material (eg, surgical clips, drains, catheters, stents, sponges)
3. Gas (eg, in abscess; necrotic tumor; sequela of tumor embolization or biopsy)

4. Parasites in bile ducts (eg, Clonorchis; Ascaris; Fasciola) (may also show linear shadowing)

5. Parasitic disease in liver parenchyma (eg, hydatid disease; amebic abscess; pentastomiasis (Armillifer infection)

6. Refractile artifacts (eg, junction of vessels; gallbladder neck)

\section{References}

1. Gore RM: Liver: Differential diagnosis. In Gore RM, Levine MS (eds): Textbook of Gastrointestinal Radiology. (ed 2) Philadelphia: WB Saunders, 2000, pp 1712-1727

2. Parulekar SG, Bree RL: Liver. In: McGahan JP, Goldberg BB (eds): Diagnostic Ultrasound. Philadelphia: LippincottRaven, 1997, chapter 21

3. Withers CW, Wilson SR: The liver. In: Rumack CM, Wilson SR, Charboneau JW (eds): Diagnostic Ultrasound. St. Louis: Mosby, 1998, pp 87-154

\section{Gamut G-180}

\section{FOCAL LOW DENSITY (DECREASED ATTENUATION) LIVER LESION (NONENHANCED CT)}

\section{COMMON}

1. Abscess (pyogenic, amebic, or fungal)

2. Cyst (eg, congenital; epithelial; posttraumatic; hydatid)

3. Focal fatty infiltration (focal steatosis)

4. Focal nodular hyperplasia

5. Hemangioma

6. Hematoma; laceration (posttraumatic)

7. Hepatocellular adenoma

8. Hepatocellular carcinoma (hepatoma)

9. Hydatid disease (Echinococcus granulosis and E. multilocularis)

10. Lymphoma

11. Metastasis (esp. from carcinoma of lung, breast, colon, kidney) 


\section{UNCOMMON}

1. Angiosarcoma of liver (may have high density regions with hemorrhage)

2. Biliary cystadenoma, cystadenoarcinoma (near water density on CT)

3. Biloma

4. Caroli disease

5. Cholangiocarcinoma

6. Choledochal cyst

7. Fibrolamellar carcinoma

8. Fungus disease with multiple microabscesses (eg, Candida; Cryptococcus)

9. Hemangioendothelioma

10. Hepatoblastoma

11. Infarct

12. Mesenchymal hamartoma

13. Multiple bile duct hamartoma

14. Polycystic liver disease

15. Radiation therapy (fatty replacement)

16. Schistosomiasis

17. Von Hippel-Lindau disease

\section{References}

1. Mathieu D, Bruneton JN, Drouillard J, et al: Hepatic adenomas and focal nodular hyperplasia: dynamic CT study. Radiology 1986;160:53-58

2. Slone RM, Fisher AJ: Pocket Guide to Body CT Differential Diagnosis. New York: McGraw-Hill, 1999, pp 212-214

\section{Gamut G-181}

\section{FOCAL LOW DENSITY LIVER LESION (POSTCONTRAST CT)}

\section{COMMON}

1. Abscess (pyogenic, amebic, or fungal)

2. Cyst (eg, congenital; epithelial; posttraumatic; hydatid)

3. Focal fatty infiltration (focal steatosis)

4. Hemangioma, giant

5. Hematoma

6. Hydatid disease (Echinococcus granulosis and E. multilocularis)
7. Metastasis (esp. from carcinoma of lung, breast, colon, kidney)

8. Regenerating nodules

\section{UNCOMMON}

1. Biloma

2. Caroli disease

3. Cholangiocarcinoma (late enhancement)

4. Fungus disease with multiple microabscesses (eg, Candida; Cryptococcus)

5. Lymphangioma

6. Polycystic liver disease

7. Radiation therapy (fatty replacement)

8. Schistosomiasis

9. Von Hipple-Lindau disease

\section{Reference}

1. Slone RM, Fisher AJ: Pocket Guide to Body CT Differential Diagnosis. New York: McGraw-Hill, 1999, pp 215-216

\section{Gamut G-182}

\section{FOCAL HIGH DENSITY LIVER LESION (NONENHANCED CT)}

1. Granuloma (esp. tuberculoma)

2. Hematoma, subcapsular or intrahepatic (first few days)

3. Hepatic tumor (eg, hepatocellular carcinoma \{hepatoma ; hepatoblastoma; hemangioendothelioma)

4. Hepatocellular adenoma with acute hemorrhage

5. Hydatid disease (old healed, calcified E. granulosus cyst; Echinococcus multilocularis-with calcification)

6. Metastasis with calcification (eg, from colon, rectum, stomach, ovary)

\section{References}

1. Bressler EL, et al: Hypervascular hepatic metastases: CT evaluation. Radiology 1987; 162:49-51

2. Chapman S, Nakielny R: Aids to Radiological Differential Diagnosis. (ed 2) London, Bailliere Tindall, 1990, p 235. 
3. Ros PR, Rosado de Christenson ML, Buetow PC, et al: Image interpretation session: 1997. Radiographics 1998;18: 199-202
4. Scatarige JC, Fishman EK, Saksouk FA, Siegelman SS: Computed tomography of calcified liver masses. J Comput Assist Tomogr 1983; 7:83-89

\section{Gamut G-184-S}

\section{MRI CHARACTERISTICS OF VARIOUS LIVER LESIONS}

\author{
1. Adenoma \\ 2. Focal nodular hyperplasia \\ central scar \\ margins \\ 3. Hemangioma \\ 4. Hemochromatosis/ \\ Iron deposition \\ 5. Hepatocellular carcinoma
}

6. Metastasis

7. Regenerating nodule
T1W

Hyperintense

Hypointense

Isointense

Hypointense

Hypointense

Hypo-, iso-, or hyperintense (due to fat degeneration)

Hypointense

Hypo- or insointense
T2W

Hypointense

Gadolinium

Hyperintense+

Hyperintense

Hyperintense ++

Hypointense++

Hyperintense

Hyperintense

Hypointense

\section{References}

1. Chapman S, Nakielny R: Aids to Radiological Differential Diagnosis. (ed 3) London: Saunders, 1995, p 279

2. Choi BI, Takayasu K, Han MC: Small hepatocellular carcinomas and associated nodular lesions of the liver: pathology, pathogenesis and imaging findings. AJR 1993;160:1177-1187

3. Vilgrain V, Flejou J-F, Arrive L: Focal nodular hyperplasia of the liver: MR imaging and pathologic correlation in 37 patients. Radiology 1992;184:699-703

\section{Gamut G-185 LIVER LESIONS WITH CIRCUMFERENTIAL RIM ON MRI}

\section{HYPOINTENSE RIM ON T1-WEIGHTED IMAGE}

1. Abscess, pyogenic or amebic (concentric rims; rim of collagen)

2. Hematoma, chronic (hemosiderin)

3. Hepatocellular carcinoma (hepatoma) (pseudocapsular rim is thin)

4. Hydatid cyst (thick, homogeneous rim; no perilesional edema)

\section{HYPOINTENSE RIM ON T2-WEIGHTED IMAGE}

1. Abscess, pyogenic or amebic (one or two concentric rings of mixed signal intensity)

2. Hematoma, subacute to chronic (white rim also seen on T1-weighted images)

3. Hydatid cyst (thick, homogeneous rim; no perilesional edema; high signal cyst contents on T2)

4. Metastasis (peritumoral edema with double ring pattern)

\section{NO RIM}

1. Cavernous hemangioma

2. Cyst, simple 
3. Focal nodular hyperplasia

4. Hepatocellular adenoma

\section{References}

1. Elizondo G, Weissleder R, Stark DD, et al: Amebic liver abscess-diagnosis and treatment evaluation with MR imaging. Radiology 1987; 165:795-800

2. Gore RM: Liver: Differential diagnosis. In Gore RM, Levine MS (eds): Textbook of Gastrointestinal Radiology. (ed 2) Philadelphia: WB Saunders, 2000, pp 1712-1727

3. Hahn PF, Stark DD, Saini S, et al: The differential diagnosis of ringed hepatic lesions in MR imaging. AJR 1990;154: 287-290

4. Hoff FL, Aisen AM, Walden ME, Glazer GM: MR imaging in hydatid disease of the liver. Gastrointest Radiol 1987;12: 39-42

5. Rummeny E, Weissleder R, Stark DD, et al: Primary liver tumors: diagnosis by MR imaging. AJR 1989;152:63-72

6. Wittenberg J, Stark DD, Forman BH, et al: Differentiation of hepatic metastases from hepatic hemangiomas and cysts by using MR imaging. AJR 1988;151:79-84

\section{Gamut G-186}

\section{FOCAL HIGH SIGNAL INTENSITY LIVER LESION ON T1-WEIGHTED MR IMAGES}

1. [Contrast agent (eg, gadolinium; lipiodol)]

2. Dysplastic nodule(s)

3. Fatty lesion (eg, lipoma; angiomyolipoma; focal fatty deposit; surgical defect packed with omental fat; occasional hepatoma with fatty degeneration)

4. Focal nodular hyperplasia

5. Hematoma or hemorrhage, acute (eg, trauma; blood dyscrasia; anticoagulants)

6. Hepatocellular adenoma

7. Hepatocellular carcinoma (hepatoma)

8. Metastasis (esp. melanoma)

9. Normal signal intensity liver surrounded by low signal intensity liver (eg, hemochromatosis; hemosiderosis; cirrhosis with regenerating nodules; edema)
10. Proteinaceous material in dependent portion of abscess or hematoma

11. [Pulsation artifact from aorta]

[ ] This condition does not actually cause the gamuted imaging finding, but can produce imaging changes that simulate it.

\section{References}

1. Chapman S, Nakielny R: Aids to Radiological Differential Diagnosis. (ed 3) London:WB Saunders, 1995, p 280

2. Gore RM: Liver: Differential diagnosis. In Gore RM, Levine MS (eds): Textbook of Gastrointestinal Radiology. (ed 2) Philadelphia: WB Saunders, 2000, pp 1712-1727

3. Lee MJ, Hahn PF, Saini S, Mueller PR: Differential diagnosis of hyperintense liver lesions on T1W MR images. AJR 1992;159:1017-1020

4. Mathieu D, Paret M, Mahfouz AE, et al.: Hyperintense benign liver lesions on spin-echo T1-weighted MR images: pathologic correlations. Abdom Imaging 1997;22:410-417

5. Mergo PJ, Ros PR: Benign lesions of the liver. Radiol Clin North Am 1998;36:319-332

\section{Gamut G-187}

\section{DAMPING OF HEPATIC VEIN DOPPLER WAVEFORM (US)}

1. Budd-Chiari syndrome

2. Cirrhosis

3. Extrinsic compression of hepatic veins

4. Passive hepatic congestion

5. Various parenchymal abnormalities of liver

\section{Reference}

1. Gore RM, Marn CS, Baron RL: Vascular disorders of the liver and splanchnic circulation. In: Gore RM, Levine MS (eds): Textbook of Gastrointestinal Radiology. (ed 2) Philadelphia: WB Saunders, 2000, pp 1639-1668 


\section{Gamut G-188}

\section{HEPATIC VEIN DILATATION}

\section{COMMON}

1. Constrictive pericarditis

2. Inferior vena cava obstruction or thrombus

3. Normal with Valsalva maneuver in young patient

4. Right-sided congestive heart failure

5. Thrombus in hepatic vein

6. Tricuspid atresia or stenosis

\section{UNCOMMON}

1. Right atrial tumor

\section{Reference}

1. Gore RM, Marn CS, Baron RL: Vascular disorders of the liver and splanchnic circulation. In: Gore RM, Levine MS (eds): Textbook of Gastrointestinal Radiology. (ed 2) Philadelphia: WB Saunders, 2000, pp 1639-1668

\section{Gamut G-189}

\section{HEPATIC VEIN THROMBOEMBOLISM OR OBSTRUCTION (BUDD-CHIARI S.) (INCL. OBSTRUCTION OF UPPER INFERIOR VENA CAVA)}

\section{COMMON}

1. Cirrhosis

2. Heart failure

3. Idiopathic

4. Neoplasm invading inferior vena cava (esp. renal cell carcinoma; hepatocellular carcinoma)

5. Thrombophlebitis

\section{UNCOMMON}

1. Alkaloid ingestion (bush tea disease)

2. Behçet syndrome
3. Coagulopathy (incl. polycythemia vera; sickle cell disease)

4. Iatrogenic (eg, catheterization; vena cavography)

5. Infection of liver (eg, aspergillosis)

6. Intravenous web

7. Metastasis, hepatic

8. Neoplastic compression (eg, carcinoma of pancreas; retroperitoneal sarcoma; lymphoma ${ }_{\mathrm{g}}$ )

9. Oral contraceptive use

10. Parasitic disease (eg, amebic liver abscess; schistosomiasis; hydatid disease)

11. Postoperative (eg, splenectomy)

12. Pregnancy; postpartum

13. Trauma

\section{References}

1. Deutsch V, Rosenthal T, Adar R, et al: Budd-Chiari syndrome. AJR 1972;116:430-439

2. Gore RM, Marn CS, Baron RL: Vascular disorders of the liver and splanchnic circulation. In: Gore RM, Levine MS: Textbook of Gastrointestinal Radiology. (ed 2) Philadelphia: WB Saunders, 2000, pp 1639-1668

3. Henderson JM, Boyer TD: Budd-Chiari syndrome. In: Zakim D, Boyer TD: Hepatology. (ed 3) Philadelphia: WB Saunders, 1996

4. Young RC: The Budd-Chiari syndrome caused by aspergillus. Arch Intern Med 1969;124:754-757

\section{Gamut G-190}

\section{PORTAL VEIN THROMBOSIS OR OBSTRUCTION*}

\section{COMMON}

1. Cirrhosis plus portal hypertension

2. Extrinsic compression or invasion by carcinoma of pancreas or stomach; lymphadenopathy of porta hepatis

3. Hepatocellular carcinoma (hepatoma) or cholangiocarcinoma (tumor thrombus or invasion)

4. Hypercoagulable state (eg, blood dyscrasia; clotting disorder; polycythemia vera; protein c or s deficiency; paroxysmal nocturnal hemoglobinuria)

(continued) 
5. Iatrogenic (eg, TIPS; umbilical venous catheterization; estrogen therapy; oral contraceptive use)

6. Idiopathic

7. Neonatal sepsis; perinatal omphalitis

8. Pancreatitis

9. Postoperative (esp. postsplenectomy)

10. Schistosomiasis (periportal fibrosis causing presinusoidal intrahepatic obstruction)

11. Sclerosing cholangitis

\section{UNCOMMON}

1. Behçet $S$.

2. [Budd-Chiari S. causing reversal of portal venous blood return]

3. Dehydration, severe

4. Liver transplantation

5. Portal phlebitis (from abdominal infection)

6. Postpartum

7. Trauma

* May result in cavernous transformation of portal vein.

[ ] This condition does not actually cause the gamuted imaging finding, but can produce imaging changes that simulate it.

\section{References}

1. Dähnert W; Radiology Review Manual. (ed 4) Baltimore: Williams \& Wilkins, 1999, p 610

2. Gore RM, Marn CS, Baron RL: Vascular disorders of the liver and splanchnic circulation. In: Gore RM, Levine MS: Textbook of Gastrointestinal Radiology. (ed 2) Philadelphia: WB Saunders, 2000, pp 1639-1668

3. Mitchel DG, Nazarian LN: Hepatic vascular disease: CT and MRI. Semin US, CT, MR 1995;16:49-68

\section{Gamut G-191}

\section{PORTAL HYPERTENSION}

\section{COMMON}

1. Cardiac (eg, heart failure; constrictive pericarditis)

2. Cirrhosis (incl. hepatitis; liver atrophy)
3. Pancreatic disease with portal obstruction (eg, neoplasm; pancreatitis)

4. Portal vein thrombosis

5. Schistosomiasis (periportal fibrosis causing presinusoidal intrahepatic obstruction)

6. Splenic vein thrombosis

\section{UNCOMMON}

1. Alagille S. (arteriohepatic S.)

2. Alpha 1-antitrypsin deficiency

3. Banti S.

4. Budd-Chiari S.

5. Caroli disease

6. [Cavernous transformation of portal vein]

7. Cholesterol ester storage disease

8. Cruveilhier-Baumgarten S.

9. Cystic fibrosis (mucoviscidosis)

10. Fatty change in liver (steatosis) (See G-144)

11. Gaucher disease

12. Glycogen storage disease, types III and IV

13. Hemochromatosis; multiple transfusion effect

14. Hepatic fibrosis-renal cystic disease

15. Increased portal flow (eg, splenomegaly; splenic, mesenteric, or hepatic arteriovenous fistula)

16. Nodular regenerative hyperplasia

17. Osler-Weber-Rendu S.

18. Polycythemia vera

19. Retroperitoneal inflammatory disease

20. Tropical splenomegaly S.

21. Wilson disease

[ ] This condition does not actually cause the gamuted imaging finding, but can produce imaging changes that simulate it.

\section{References}

1. Baron RL, Gore RM: Diffuse liver disease. In: Gore RM, Levine MS: Textbook of Gastrointestinal Radiology. (ed 2) Philadelphia: WB Saunders, 2000, pp 1590-1638 
2. Sutton D, Young JWR: A Short Textbook of Clinical Imaging. London: Springer-Verlag, 1990, p 252

3. Taybi H, Lachman RS: Radiology of Syndromes, Metabolic Disorders, and Skeletal Dysplasias. (ed 4) St. Louis: MosbyYear Book, 1996, pp 973-974

\section{Gamut G-192}

\section{GAS IN THE PORTAL VEINS}

\section{COMMON}

1. Abscess, abdominal $_{\mathrm{g}}$ or pelvic (eg, gas abscess of pancreas; diverticulitis)

2. [Gas in the biliary tract]

3. Iatrogenic (eg, catheterization of umbilical or mesenteric artery or vein; percutaneous abscess drainage; hydrogen peroxide enema or gastric lavage; post-hepatic artery embolization)

4. Mechanical bowel obstruction with ischemia (incl. closed loop obstruction)

5. Mesenteric ischemia or occlusion and bowel infarction (See G-69)

6. Necrotizing enterocolitis

\section{UNCOMMON}

1. Acute gastric dilatation

2. Carcinoma of colon, necrotic

3. Corrosive ingestion causing gastritis

4. Diabetic acidosis or coma

5. Emphysematous cholecystitis

6. Erythroblastosis fetalis

7. Gastric emphysema

8. Hemorrhagic pancreatitis
9. Neonatal gastroenteritis

10. Peptic ulcer eroding into mesenteric vein

11. Postcolonoscopy or post-air contrast barium enema in inflammatory bowel disease (eg, diverticulitis; Crohn's disease; ulcerative colitis;)

12. Postoperative (eg, bowel resection; operation for congenital bowel obstruction)

13. Pseudomembranous colitis

14. Sepsis

15. Toxic megacolon

[ ] This condition does not actually cause the gamuted imaging finding, but can produce imaging changes that simulate it.

\section{References}

1. Bach MC, Anderson LG, Martin TA: Gas in the hepatic portal venous system. A diagnostic clue to an occult intraabdominal abscess. Arch Intern Med 1982; 142:1725-1726

2. Benson MB: Adult survival with intrahepatic portal venous gas secondary to acute gastric dilatation, with a review of portal venous gas. Clin Radiol 1985; 36:441-443

3. Eisenberg RL: Gastrointestinal Radiology: A Pattern Approach. (ed 3) Philadelphia: Lippincott, 1996, pp 894-899

4. Gore RM: Liver: differential diagnosis. In: Gore RM, Levine MS: Textbook of Gastrointestinal Radiology. (ed 2) Philadelphia: WB Saunders, 2000, pp 1712-1726

5. Kirks DR, O'Byrne SA: The value of the lateral abdominal roentgenogram in the diagnosis of neonatal hepatic portal venous gas (HPVG). AJR 1974;122:153-158

6. Merritt CRB, Goldsmith JP, Shary MJ: Sonographic detection of portal venous gas in infants with NEC. Am J Roentgenol 1984;143:1059

7. Mindelzun R, McCort JJ: Hepatic and perihepatic radiolucencies. Radiol Clin North Am 1980; 18:221-238

8. Sisk PB: Gas in the portal venous system. Radiology 1961;77:103-106

9. Wiot JF, Felson B: Gas in the portal venous system. AJR 1961;86:920-929 


\section{Gamut G-193}

\section{PERIPORTAL HYPOECHOGENICITY ON US/PERIPORTAL LOW DENSITY ON CT}

1. AIDS-related cholangitis

2. Cholangitis

3. Congestive hepatomegaly

4. Liver transplant rejection (occasionally seen in nonrejecting liver transplant)

5. Malignant lymphatic obstruction

6. Schistosomiasis mansoni

7. Trauma, blunt abdominal

8. Viral hepatitis

\section{References}

1. Chapman S, Nakielny R: Aids to Radiological Differential Diagnosis. (ed 3) London: WB Saunders, 1995, p 272

2. Fataar S, Bassiony H, Satyanath S, et al: CT of hepatic Schistosomiasis mansoni. AJR 1985;145:63-66

3. Kaplan SB, Sumkin JH, Campbell WL, et al: Periportal lowattenuation areas on CT: value as evidence of liver transplant rejection. AJR 1989;152:285-287

4. Shanmuganathan K, Mirvis SE, Amorosa M: Periportal low density on CT in patients with blunt trauma: association with elevated venous pressure. AJR 1993;160:279-283

5. Siegel MJ, Herman TE: Periportal low attenuation at CT in childhood. Radiology 1992;183:685-688

6. Wechsler RJ, Munoz SJ, Needleman L, et al: The periportal collar: a CT sign of liver transplant rejection. Radiology 1987;165:57-60

\section{Gamut G-194}

\section{PROMINENT PERIPORTAL ECHOES (STARRY SKY LIVER) IN ADULTS (US)}

\section{COMMON}

1. Air in biliary tree (pneumobilia)

2. Cholangiocarcinoma

3. Cholangitis

4. Cholecystitis, acute or chronic
5. Hepatitis

6. Hepatocellular carcinoma (hepatoma)

7. Recurrent pyogenic cholangitis (Oriental cholangiohepatitis)

8. Sclerosing cholangitis

\section{UNCOMMON}

1. Cystic fibrosis (mucoviscidosis)

2. Embolism of oily contrast medium after lymphangiogram

3. Infectious mononucleosis

4. Kaposi sarcoma of liver

5. Lymphoma ${ }_{\mathrm{g}}$; Burkitt lymphoma; leukemia

6. Periportal fibrosis (incl. schistosomiasis)

7. Right heart failure

8. Toxic shock syndrome

\section{References}

1. Gore RM: Liver: Differential diagnosis. In Gore RM, Levine MS (eds): Textbook of Gastrointestinal Radiology. (ed 2) Philadelphia: WB Saunders, 2000, pp 1712-1727

2. Williamson MR: Abdominal ultrasound. In: Essentials of Ultrasound. Philadelphia: WB Saunders, 1996, p 81

\section{Gamut G-195}

\section{PROMINENT PERIPORTAL ECHOES IN NEONATES ON ULTRASOUND}

\section{COMMON}

1. Acute hepatitis

2. Biliary atresia

3. [Idiopathic; transient (disappears within a year)]

4. Idiopathic neonatal jaundice

\section{UNCOMMON}

1. [Air in portal venous system]

2. Alpha-1-antitrypsin deficiency

3. Cytomegalovirus infection

[ ] This condition does not actually cause the gamuted imaging finding, but can produce imaging changes that simulate it. 


\section{Reference}

1. Gore RM: Liver: Differential diagnosis. In Gore RM, Levine MS (eds): Textbook of Gastrointestinal Radiology. (ed 2) Philadelphia: WB Saunders, 2000, pp 1712-1727

\section{Gamut G-196}

\section{INCREASED PERIPORTAL SIGNAL INTENSITY ON MRI}

1. Acute hepatitis

2. AIDS-related cholangitis

3. Cholangiocarcinoma

4. Cholangitis

5. Cirrhosis

6. Obstructive jaundice

\section{Reference}

1. Gore RM: Liver: Differential diagnosis. In Gore RM, Levine MS (eds): Textbook of Gastrointestinal Radiology. (ed 2) Philadelphia: WB Saunders, 2000, pp 1712- 1727

\section{Gamut G-197}

\section{SPLENOMEGALY: \\ A CLASSIFICATION (See G-198)}

\section{BLOOD DYSCRASIA}

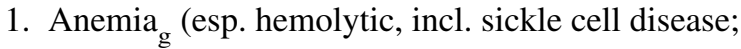 thalassemia; hereditary spherocytosis; pyruvate kinase deficiency)
2. Dysgammaglobulinemia
3. Extramedullary hematopoiesis
4. Hemochromatosis
5. Leukemia (esp. chronic myelogenous)
6. Myelofibrosis (hypersplenism)
7. Osteopetrosis
8. Polycythemia vera

9. Thrombotic thrombocytopenic purpura

10. Waldenström macroglobulinemia

\section{INFECTION}

1. Bacterial (eg, miliary tuberculosis; tularemia; subacute bacterial endocarditis; septicemia; typhoid fever; brucellosis; syphilis; pyogenic abscess; tropical splenic abscess)

2. Chronic granulomatous disease of childhood

3. Fungal (esp. histoplasmosis; candidiasis)

4. Parasitic disease (eg, malaria; schistosomiasis; hydatid disease; kala-azar \{leishmaniasis $\}$ )

5. Rickettsial (eg, typhus)

6. Viral (eg, viral hepatitis; AIDS; infectious mononucleosis; cytomegalovirus infection; herpes simplex; rubella)

\section{NEOPLASM}

1. Benign neoplasm (eg, fibroma; hamartoma)

2. Cyst, epidermoid (simple, primary); dermoid; posttraumatic; hydatid

3. Hemangioma; cystic lymphangioma

4. Lymphoma

5. Metastases (esp. from carcinoma of breast, lung, colon, ovary; melanoma)

6. Sarcoma (esp. angiosarcoma)

\section{PORTAL HYPERTENSION}

1. Cirrhosis, nutritional or alcoholic

2. Congestive splenomegaly (Banti syndrome)

3. Schistosomiasis

4. Splenic or portal vein obstruction (eg, thrombosis; pancreatic neoplasm; lymphadenopathy)

\section{STORAGE DISEASES}

1. Gaucher disease

2. Glycogen storage disease

3. Langerhans cell histiocytosis

4. Mucopolysaccharidoses (incl. gargoylism); mucolipidoses (See J-4)

5. Niemann-Pick disease 


\section{TRAUMA}

1. Hematoma (subcapsular; intrasplenic; perisplenic)

2. Hemorrhagic pseudocyst

\section{OTHER}

1. Alpha-1-antitrypsin deficiency

2. Amyloidosis

3. Congenital syndromes (See G-198)

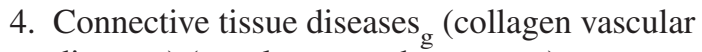
diseases) (esp. lupus erythematosus)

5. Cystic fibrosis (mucoviscidosis)

6. Diabetes

7. Heart failure

8. Hemodialysis

9. Infarction

10. Rheumatoid arthritis (Felty S.); juvenile rheumatoid arthritis; juvenile chronic arthritis (Still's disease)

11. Sarcoidosis

12. Tropical splenomegaly

\section{References}

1. Ayers AB: The spleen. In: Grainger RG, Allison DJ: Diagnostic Radiology. An Anglo-American Textbook of Imaging. (ed 2) Edinburgh: Churchill Livingstone, 1992, vol 3, p 2408

2. Dähnert W: Radiology Review Manual. (ed 4) Baltimore, Williams \& Wilkins, 1999, p 556

3. Gore RM: Spleen: Differential diagnosis. In: Gore RM, Levine MS: Textbook of Gastrointestinal Radiology. (ed 2) Philadelphia: WB Saunders, 2000, pp 1925-1928

4. Siegel MJ: Pediatric Body CT. Philadelphia: Lippincott Williams \& Wilkins, 1999

5. Teplick JG, Haskin ME: Roentgenographic Diagnosis. (ed 3) Philadelphia: WB Saunders, 1976

\section{Gamut G-198}

\section{CONGENITAL SYNDROMES WITH SPLENOMEGALY}

\section{COMMON}

1. Anemia ${ }_{g}$ (eg, sickle cell disease; thalassemia; spherocytosis; pyruvate kinase deficiency)

2. Chronic granulomatous disease of childhood

3. Dysgammaglobulinemia

4. Fetal infection (eg, rubella; cytomegalovirus; herpes simplex)

5. Gaucher disease; Niemann-Pick disease

6. Glycogen storage disease, type I (von Gierke disease) and type III

7. Hemochromatosis

8. Langerhans cell histiocytosis ${ }_{\mathrm{g}}$

9. Mucopolysaccharidoses; mucolipidoses; $\mathrm{GM}_{1}$ gangliosidosis (See J-4)

\section{UNCOMMON}

1. Aase $\mathrm{S}$.

2. Alpha-1-antitrypsin deficiency

3. Aspartylglucosaminuria

4. Beckwith-Wiedemann S.

5. Chédiak-Higashi S.

6. Cholesterol ester storage disease

7. Cogan S.

8. Cruveilhier-Baumgarten S.

9. Cystinosis

10. Ethanolaminosis

11. Farber disease (lipogranulomatosis)

12. Felty S.

13. Fucosidosis; galactosialidosis; mannosidosis

14. Hepatic fibrosis-renal cystic disease

15. Hyperlipoproteinemia

16. Infantile multisystem inflammatory disease (NOMID)

17. Lipoatrophic diabetes

18. Osteopetrosis

19. POEMS S.

20. Sea-blue histiocyte $S$. 
21. Tyrosinemia, type I

22. Vaquez-Osler $\mathrm{S}$.

23. Wilson disease

24. Wolman disease

\section{Reference}

1. Taybi H, Lachman RS: Radiology of Syndromes, Metabolic Disorders, and Skeletal Dysplasias. (ed 4) St. Louis: MosbyYear Book, 1996, p 961

\section{Gamut G-199}

\section{SPLENOMEGALY WITH NORMAL ECHOGENICITY (US)}

1. Congestion from portal hypertension

2. Felty syndrome

3. Hemolysis

4. Hereditary spherocytosis

5. Infection

6. Juvenile chronic arthritis (Still's disease) (eg, juvenile rhematoid arthritis)

7. Myelofibrosis

8. Leukemia, myelogenous

9. Parasitic disease (eg, malaria; leishmaniasis \{kalaazar\}; schistosomiasis)

10. Polycythemia vera

11. Sickle cell disease (early)

12. Wilson disease

\section{Reference}

1. Gore RM: Spleen: Differential diagnosis. In: Gore RM, Levine MS: Textbook of Gastrointestinal Radiology. (ed 2) Philadelphia: WB Saunders, 2000, pp 1925-1928

\section{Gamut G-200}

\section{SPLENOMEGALY WITH DIFFUSE HYPOECHOIC PATTERN (US)}

1. Congestion from portal hypertension

2. Leukemia (esp. chronic lymphocytic)

3. Lymphoma

4. Multiple myeloma

5. Noncaseating granulomatous disease

\section{Reference}

1. Gore RM: Spleen: Differential diagnosis. In: Gore RM, Levine MS: Textbook of Gastrointestinal Radiology. (ed 2) Philadelphia: WB Saunders, 2000, pp 1925-1928

\section{Gamut G-201}

\section{SPLENOMEGALY WITH DIFFUSE HYPERECHOIC PATTERN (US)}

\section{COMMON}

1. Hematoma

2. Infection (eg, malaria; brucellosis; tuberculosisesp. miliary)

3. Leukemia (acute lymphocytic, chronic lymphocytic, or myelogenous after chemotherapy or radiation therapy)

4. Lymphoma ${ }_{\mathrm{g}}$

5. Myelofibrosis (hypersplenism)

\section{UNCOMMON}

1. Dysgammaglobulinemia

2. Hereditary spherocytosis

3. Malignant neoplasm (esp. angiosarcoma)

4. Metastasis

5. Polycythemia

6. Portal vein thrombosis

7. Sarcoidosis 


\section{References}

1. Gore RM: Spleen: Differential diagnosis. In: Gore RM, Levine MS: Textbook of Gastrointestinal Radiology. (ed 2) Philadelphia: WB Saunders, 2000, pp 1925-1928

2. Mathieson JR, Cooperberg PL: The Spleen. In Rumack CM, Wilson SR, Charboneau JW (eds): Diagnostic Ultrasound. (ed 2) St. Louis: Mosby, 1998, pp 155-174

3. Siler J, Hunter TB, Weiss J, et al: Increased echogenicity of the spleen in benign and malignant disease. AJR 1980; 134:1011-1014

\section{Gamut G-202}

\section{SMALL OR NONVISUALIZED SPLEEN}

1. Asplenia S.; polysplenia S.

2. Atrophy

3. Hereditary hypoplasia

4. Inflammatory bowel disease

5. Postinfarction (esp. sickle cell disease)

6. Postradiation therapy

7. Sickle cell disease

8. Traumatic fragmentation

9. Wandering spleen

\section{References}

1. Dähnert W: Radiology Review Manual. (ed 4) Baltimore, Williams \& Wilkins, 1999, p 556

2. Gore RM: Spleen: Differential diagnosis. In: Gore RM, Levine MS: Textbook of Gastrointestinal Radiology. (ed 2) Philadelphia: WB Saunders, 2000, pp 1925-1928

\section{Gamut G-203}

\section{SPLENIC CALCIFICATION-SOLITARY}

\section{COMMON}

1. Splenic artery aneurysm

2. Splenic artery atherosclerosis

\section{UNCOMMON}

1. Abscess, healed (eg, pyogenic; Candida; tuberculous)

2. Capsular ascites ("zuckerguss" spleen)

3. Cyst (eg, congenital; dermoid; epidermoid; hydatid; posttraumatic)

4. Granuloma (esp. tuberculosis)

5. Hamartoma

6. Hemangioma; phlebolith

7. Hematoma, healed

8. Infarct, healed

9. Metastasis

\section{References}

1. Eisenberg RL: Gastrointestinal Radiology: A Pattern Approach. (ed 3) Philadelphia: Lippincott, 1996, pp 991-997

2. Gore RM: Spleen: Differential diagnosis. In: Gore RM, Levine MS: Textbook of Gastrointestinal Radiology. (ed 2) Philadelphia: WB Saunders, 2000, pp 1925-1928

\section{Gamut G-204}

\section{SPLENIC CALCIFICATIONS-MULTIPLE}

\section{COMMON}

1. Granulomas, healed (eg, tuberculosis; histoplasmosis)

2. Phleboliths; hemangioma

3. Splenic artery

\section{UNCOMMON}

1. AIDS (healed Pneumocystis carinii infection)

2. Brucellosis 
3. Hematomas

4. Hemochromatosis

5. Hemosiderosis

6. Infarcts

7. Metastases

8. Parasitic disease, esp. pentastomiasis (Armillifer infection); hydatid disease

9. Sarcoidosis

10. Sickle cell disease (stippled)

11. [Thorotrast residual]

[ ] This condition does not actually cause the gamuted imaging finding, but can produce imaging changes that simulate it.

\section{References}

1. Baker SR, Cho KC: The Abdominal Plain Film with Correlative Imaging. Norwalk, CT: Appleton \& Lange, 1998

2. Eisenberg RL: Gastrointestinal Radiology: A Pattern Approach. (ed 3) Philadelphia: Lippincott, 1996, pp 991-997

3. Gore RM: Spleen: Differential diagnosis. In: Gore RM, Levine MS: Textbook of Gastrointestinal Radiology. (ed 2) Philadelphia: WB Saunders, 2000, pp 1925-1928

\section{Gamut G-205}

\section{SOLITARY LESION OF THE SPLEEN (US, CT, MRI)}

\section{COMMON}

1. Aneurysm of splenic artery

2. Cyst (congenital; epidermoid; dermoid or teratoma; hydatid; traumatic)

3. Hemangioma, cavernous or capillary

4. Hematoma (posttraumatic)

5. Infarct (esp. sickle cell disease)

6. Lymphoma ${ }_{\mathrm{g}}$; leukemia

\section{UNCOMMON}

1. Abscess (pyogenic; Candida; Pneumocystis carinii)

2. Arteriovenous malformation

3. Cystic lymphangioma

4. Hamartoma

5. Hemangiosarcoma
6. Metastasis (esp. from carcinoma of breast, lung, colon, ovary; melanoma)

7. Peliosis

8. Pseudocyst (secondary to pancreatitis)

9. Sarcoidosis

10. Splenic sequestration crisis

\section{References}

1. Dachman AH, Ros PR, Murari PJ, et al: Nonparasitic splenic cysts: A report of 52 cases with radiologic-pathologic correlation. AJR 1986;147:537-542

2. Guermazi A, Brice P, deKerviler E, et al: Extranodal Hodgkin disease: spectrum of disease. RadioGraphics 2001; 21:161-179

3. Mathieson JR, Cooperberg PL: Chapter 5. In: Rumack CM, Wilson SR, Charboneau JW (eds): Diagnostic Ultrasound. St. Louis: Mosby, 1998, pp 162-171

4. Reddy SC: Hemangiosarcoma of the spleen: Helical computed tomography features. South Med J 2000;93:825-827

5. Slone RM, Fisher AJ: Pocket Guide to Body CT Differential Diagnosis. New York: McGraw-Hill, 1999, pp 237-238

6. Solviati L, Bossi MC, Bellotti E, et al: Focal lesions in the spleen: Sonographic patterns and guided biopsy. AJR 1983; 140:59-65

7. Urrutia M, Mergo PJ, Ros LH, Torres GM, Ros PR: Cystic masses of the spleen: radiologic-pathologic correlation. RadioGraphics 1996;16:107-129

\section{Gamut G-206}

\section{FOCAL CYSTIC OR LOW DENSITY SPLENIC LESION (CT, MRI-USUALLY HYPOECHOIC ON US)}

\section{COMMON}

1. Abscess (pyogenic; Candida; Pneumocystis carinii)

2. Aneurysm of splenic artery

3. Cyst (congenital; epidermoid; dermoid or teratoma; hydatid; posttraumatic)

4. Hematoma

5. Infarction

\section{UNCOMMON}

1. Cystic lymphangioma

2. Hamartoma

3. Hemangioma, cavernous or capillary

(continued) 
4. Hemangiosarcoma

5. Metastasis

6. Peliosis

7. Pseudocyst (secondary to pancreatitis)

\section{References}

1. Dachman AH, Ros PR, Murari PJ, et al: Nonparasitic splenic cysts: A report of 52 cases with radiologic-pathologic correlation. AJR 1986;147:537-542

2. Mathieson JR, Cooperberg PL: Chapter 5. In: Rumack CM, Wilson SR, Charboneau JW (eds): Diagnostic Ultrasound. St. Louis: Mosby, 1998, pp 162-171

3. Solviati L, Bossi MC, Bellotti E, et al: Focal lesions in the spleen: Sonographic patterns and guided biopsy. AJR 1983;140:59-65

4. Urrutia M, Mergo PJ, Ros LH, Torres GM, Ros PR: Cystic masses of the spleen: radiologic-pathologic correlation. RadioGraphics 1996;16:107-129

\section{Gamut G-207}

\section{FOCAL HYPERECHOIC SPLENIC LESION (US)}

\section{COMMON}
1. Hematoma
2. Infarct
3. Lymphoma $_{\mathrm{g}}$

\section{UNCOMMON}

1. Abscess with gas bubbles

2. Hemangioma

3. Hemangiosarcoma

4. Hydatid cyst with "hydatid sand"

5. Metastasis

6. Plasmacytoma

7. Simple cyst with cholesterol crystals

\section{References}

1. Gore RM: Spleen: Differential diagnosis. In: Gore RM, Levine MS: Textbook of Gastrointestinal Radiology. (ed 2) Philadelphia: WB Saunders, 2000, pp 1925-1928
2. Mittelstaedt CA, Partain CL: Ultrasonic-pathologic classification of splenic abnormalities: Gray-scale patterns. Radiology 1980;134:697-705

3. Solviati L, Bossi MC, Bellotti E, et al: Focal lesions in the spleen: Sonographic patterns and guided biopsy. AJR 1983;140:59-65

\section{Gamut G-208 \\ DIFFUSE INCREASED SPLENIC DENSITY ON CT}

1. Fanconi anemia

2. Hemochromatosis

3. Hemosiderosis

4. Lymphoma (treated)

5. Sickle cell disease

6. Thorotrast residual

\section{References}

1. Kawashima A, Urban BA, Fishman EK: Benign lesions of the spleen. In: Gore RM, Levine MS (eds): Textbook of Gastrointestinal Radiology. (ed 2) Philadelphia: WB Saunders, 2000, pp. 1879-1904

2. Gore RM: Spleen: Differential diagnosis. In: Gore RM, Levine MS (eds): Textbook of Gastrointestinal Radiology. (ed 2) Philadelphia: WB Saunders, 2000, pp. 1925-1929

\section{COMMON}

*1. Abscesses (pyogenic)

*2. Cysts (eg, congenital; hydatid; posttraumatic)

3. Gamna-Gandy bodies

4. Granulomas, calcified

*5. Hemangiomas

*6. Hematomas

*7. Infarcts 
*8. Metastases (incl. hemorrhagic choriocarcinoma)

*9. Lymphoma $_{\mathrm{g}}$

\section{UNCOMMON}

1. Amyloidosis

2. Flow void in arteriovenous malformation or OslerWeber-Rendu S.

3. Fungemia with multifocal microabscess formation

*4. Peliosis

5. Sarcoidosis

* On T1-weighted images.

\section{References}

1. Gore RM: Spleen: Differential diagnosis. In: Gore RM, Levine MS: Textbook of Gastrointestinal Radiology. (ed 2) Philadelphia: WB Saunders, 2000, pp 1925-1928

2. Kelekis NL, Burdeny DA, Semelka RC: Spleen. In: Semelka RC, Ascher SM, Reinhold C (eds): MRI of the Abdomen and Pelvis. New York: Wiley-Liss, 1997, pp 239-256

\section{Gamut G-210 MULTIPLE SPLENIC HYPERINTENSITIES ON T2-WEIGHTED MR IMAGES}

\section{COMMON}

1. Abscesses (pyogenic)

2. Cysts (eg, congenital; hydatid; posttraumatic)

3. Hemangiomas

4. Hematomas

5. Infarcts

6. Metastases

\section{UNCOMMON}

1. Fungemia with multifocal microabscess formation

2. Lymphangioma (T1 and T2-WI)

3. Peliosis

\section{References}

1. Gore RM: Spleen: Differential diagnosis. In: Gore RM, Levine MS: Textbook of Gastrointestinal Radiology. (ed 2) Philadelphia: WB Saunders, 2000, pp 1925-1928

2. Kelekis NL, Burdeny DA, Semelka RC: Spleen. In: Semelka RC, Ascher SM, Reinhold C (eds): MRI of the Abdomen and Pelvis. New York: Wiley-Liss, 1997, pp 239-256

\section{Gamut G-211}

\section{ABNORMALITY ON SPLENIC ARTERIOGRAPHY}

\section{COMMON}

1. Accessory spleen

2. Anomalous origin of splenic or celiac artery

3. Splenic artery arteriosclerosis; aneurysm; fibromuscular hyperplasia (incl. stenosis; occlusion)

4. Splenic vein abnormality (See G-212)

\section{UNCOMMON}

1. Arteriovenous fistula; angiomatous malformation

2. Avascular mass
a. Abscess
b. Cyst (eg, dermoid; epidermoid; hydatid; post- traumatic)
c. Infarction
d. Lymphoma
e. Metastasis

3. Displacement by extrinsic mass

4. Splenic artery encasement (eg, pancreatic carcinoma)

5. Splenic laceration; pericapsular hematoma

6. Thromboembolism

7. Vascular mass (eg, metastasis, esp. melanoma; hemangiosarcoma of spleen) 


\section{Gamut G-212}

\section{SPLENIC VEIN OBSTRUCTION (US, POSTCONTRAST CT, MRI, OR ANGIO)}

\section{COMMON}

1. Lymphadenopathy (eg, lymphoma ${ }_{\mathrm{g}}$; metastases)

2. Neoplasm (eg, pancreatic; retroperitoneal sarcoma)

3. Pancreatitis (incl. pancreatic pseudocyst)

4. Thromboembolism of portal or splenic vein (esp. portal hypertension; polycythemia vera; heart failure)

\section{UNCOMMON}

1. Aneurysm of splenic artery or aorta

2. Postoperative

3. Retroperitoneal inflammation

4. Schistosomiasis

5. Trauma; hematoma

\section{References}

1. Gore RM, Marn CS, Baron RL: Vascular disorders of the liver and splanchnic circulation. In Gore RM, Levine MS (eds): Textbook of Gastrointestinal Radiology. (ed 2) Philadelphia: WB Saunders, 2000, pp 1639-1668

2. Rosch J, Dotter CT: Extrahepatic portal obstruction in childhood and its angiographic diagnosis. AJR 1971;112: 143-149

3. Yale CE, Crummy AB: Splenic vein thrombosis and bleeding esophageal varices. JAMA 1971; 217:317-320

\section{Gamut G-213}

\section{SOLID PANCREATIC LESION (US, CT, MRI)}

\section{UNCOMMON}

1. [Accessory spleen]

2. Aneurysm, thrombosed

3. Annular pancreas; pancreatic divisum

4. Hemangioma

5. Islet cell tumor (eg, insulinoma; gastrinoma; glucagonoma; somatostatinoma; VIPoma; nonfunctioning)

6. Lipoma

7. [Lymphadenopathy in celiac axis (eg, metastatic; tuberculous; Castleman disease)]

8. Lymphoma

9. Metastasis (esp. from carcinoma of breast, lung, stomach, gallbladder, kidney; melanoma)

10. Pancreaticoblastoma

11. Sarcoma

12. Serous cystadenoma (microcystic adenoma)

13. Solid and papillary epithelial neoplasm

[ ] This condition does not actually cause the gamuted imaging finding, but can produce imaging changes that simulate it.

\section{References}

1. Cheszmar JL: Pancreatic neoplasms. In: Gore RM, Levine MS (eds): Textbook of Gastrointestinal Radiology. (ed 2) Philadelphia: WB Saunders, 2000, pp 1796-1811

2. Clark LR, Jaffe MH, Choyke PL, et al: Pancreatic imaging. Radiol Clin North Am 1985;23:489-501

3. Helmberger TK, Ros LH, Baretton G, et al: Solid pancreatic lesions. Eur Radiol 1999;9(suppl 1):s195-s205

4. Sato M, Ishida H, Konno K, et al: Pancreatic metastasis: sonographic findings. Abdom Imaging 2001;26:72-75

5. Sato M, Ishida H, Konno K, et al: Pancreatic uncinate carcinoma: sonographic findings. Abdom Imaging 2001;26: 64-68

6. Scatarige JC, Horton KM, Sheth S, et al: Pancreatic parenchymal metastases: observations on helical CT. AJR 2001;176:695-701

7. Slone RM, Fisher AJ: Pocket Guide to Body CT Differential Diagnosis. New York: McGraw-Hill, 1999, pp 237-238

\section{COMMON}

1. Adenocarcinoma

2. Pancreatitis, focal 


\section{Gamut G-214 \\ CYSTIC OR LOW DENSITY PANCREATIC LESION (CT, MRI-HYPOECHOIC ON US)}

\section{COMMON}

1. Acute pancreatitis, focal

2. Chronic pancreatitis (inhomogeneous, often hyperechoic)

3. Fluid collection, peripancreatic

4. Mucinous cystadenoma or cystadenocarcinoma (macrocystic adenoma)

5. Pancreatic duct dilatation, ectasia, or obstruction (from carcinoma; calculus; stricture)

6. Pancreatic ductal adenocarcinoma (eg, duct ectatic mucinous adenocarcinoma; papillary intraductal adenocarcinoma; anaplastic adenocarcinoma)

7. Pseudocyst of pancreas

8. Serous cystadenoma (microcystic adenoma)

\section{UNCOMMON}

1. Abscess $_{\mathrm{g}}$

2. [Aneurysm or pseudoaneurysm of aorta, pancreaticoduodenal arcade, or splenic artery]

3. [Biloma]

4. [Cystic lymphangioma, retroperitoneal]

5. Cystic teratoma

6. Cysts, congenital (in adult polycystic kidney disease; Von Hippel-Lindau disease; or cystic fibrosis)

7. [Duodenal cyst or diverticulum]

8. Hemangioma

9. Hematoma

10. Hydatid cyst

11. Islet cell tumor or insulinoma, cystic

12. Liquefaction necrosis of pancreas

13. Lymphoma

14. Metastasis, cystic (eg, renal cell carcinoma; hepatoma; carcinoma of lung, breast, or ovary; melanoma)

15. Posttraumatic cyst
16. Solid and papillary epithelial neoplasm

17. [Varix of left renal vein]

[ ] This condition does not actually cause the gamuted imaging finding, but can produce imaging changes that simulate it.

\section{References}

1. Curry CA, Eng J, Horton KM, et al: CT of primary cystic pancreatic neoplasms: can CT be used for patient triage and treatment? AJR 2000;175:99-103

2. Kobayashi T, Kawabe A, Uenoyama S, et al: Macrocystic serous cystadenoma of the pancreas: case report. Abdom Imaging 2001;26:69-71

3. Ros PR, Hamrick-Turner JE, Chiechi MV, et al: Cystic pancreatic mass. Radiographics 1992;12:673-686

4. Ros LH, Helmberger T, Ros PR: Cystic pancreatic lesions. Eur Radiol 1999;9(suppl 1):s207-s215

5. Sato M, Ishida H, Konno K, et al: Pancreatic metastasis: sonographic findings. Abdom Imaging 2001:26:72-75

6. Scatarige JC, Horton KM, Sheth S, et al: Pancreatic parenchymal metastases: observations on helical CT. AJR 2001;176:695-701

7. Skolnick ML: Guide to the Ultrasound Examination of the Abdomen. New York: Springer-Verlag, 1986, pp 137-142

8. Williamson MR: Abdominal ultrasound. In: Essentials of Ultrasound. Philadelphia: WB Saunders, 1996, p 72

\section{Gamut G-215}

\section{COMPLEX PANCREATIC LESION (US)}

\section{COMMON}

1. Mucinous cystadenoma or cystadenocarcinoma (macrocystic adenoma)

2. Pseudocyst of pancreas

\section{UNCOMMON}

1. Hematoma, nonacute

2. Metastasis

3. Serous cystadenoma (microcystic adenoma)

\section{References}

1. Sato M, Ishida H, Konno K, et al: Pancreatic metastasis: sonographic findings. Abdom Imaging 2001:26:72-75

2. Skolnick ML: Guide to the Ultrasound Examination of the Abdomen. New York: Springer-Verlag, 1986, pp 137-142 


\section{Gamut G-216}

\section{INCREASED ECHOGENICITY OF PANCREAS (US)}

\section{COMMON}

1. Advanced age

2. Chronic pancreatitis

3. Fatty infiltration

4. Hemorrhagic pancreatitis

\section{UNCOMMON}

1. Adenocarcinoma of pancreas

2. Cystic fibrosis

3. Diabetes

4. Hereditary pancreatitis

5. Malabsorption

6. Pancreatic insufficiency

7. Shwachman-Diamond S.

8. Steroid ingestion

\section{References}

1. Scafidi DE, Young LW: Diseases of the pediatric pancreas. In: Gore RM, Levine MS (eds): Textbook of Gastrointestinal Radiology. (ed 2) Philadelphia: WB Saunders, 2000, pp 2162-2173

2. Williamson MR: Abdominal ultrasound. In: Essentials of Ultrasound. Philadelphia: WB Saunders, 1996, p 70

\section{Gamut G-217}

\section{FOCAL SHADOWING PANCREATIC LESION (US)}

\section{COMMON}

1. Calcifications in chronic pancreatitis

2. Calculi in pancreatic duct

\section{UNCOMMON}

1. Arterial calcification

2. Gas in pancreatic abscess
3. Gas in pancreatic pseudocyst communicating with gut

4. Serous cystadenoma (microcystic adenoma) with calcifications

5. Solid and papillary epithelial neoplasm

\section{References}

1. Gore RM: Pancreas: Differential diagnosis. In: Gore RM, Levine MS: Textbook of Gastrointestinal Radiology. (ed 2) Philadelphia: WB Saunders, 2000, pp 1836-1843

2. Skolnick ML: Guide to the Ultrasound Examination of the Abdomen. New York: Springer-Verlag, 1986, pp 137-142

\section{Gamut G-218}

\section{PANCREATIC CALCIFICATION WITHOUT MASS}

\section{COMMON}

1. Chronic pancreatitis

\section{UNCOMMON}

1. Acute pancreatitis (saponification)

2. [Aneurysm or atherosclerosis of aorta or its branches]

3. Cystic fibrosis (mucoviscidosis)

4. Hemochromatosis

5. Hemorrhage (eg, trauma; infarction)

6. Hereditary pancreatitis (large clumps of calcium)

7. Hyperparathyroidism with pancreatitis

8. Idiopathic (pancreatic duct stenosis)

9. Kwashiorkor

10. Pancreatitis due to biliary disease (eg, gallstone in common duct)

[ ] This condition does not actually cause the gamuted imaging finding, but can produce imaging changes that simulate it.

\section{References}

1. Burgener FA, Kormano M: Differential Diagnosis in Conventional Radiology. (ed 2) New York: Thieme Medical Publ, 1991, pp 538-540 
2. Eisenberg RL: Gastrointestinal Radiology: A Pattern Approach. (ed 3) Philadelphia: Lippincott, 1996, pp 997-1002

3. Ring EJ, Eaton SB, Ferrucci JT, Short WF: Differential diagnosis of pancreatic calcification. AJR 1973;177:446-452

\section{Gamut G-219 \\ PANCREATIC LESION CHARACTERIZED BY CALCIFICATION}

\section{COMMON}

1. [Aneurysm of aorta, pancreaticoduodenal arcade, or splenic artery, thrombosed]

2. Chronic pancreatitis, focal

3. Hemorrhage, intraparenchymal (eg, old abscess; hematoma; infarction)

\section{UNCOMMON}

1. Adenocarcinoma of pancreas (rarely)

2. Hemangioma (plebolith)

3. Hydatid cyst

4. Islet cell tumor

5. Lymphangioma, cavernous

6. Lymphoma (treated)

7. Metastasis (esp. mucinous adenocarcinoma of colon)

8. Mucinous cystadenoma or cystadenocarcinoma (macrocystic adenoma) (peripheral calcium)

9. Pancreaticoblastoma

10. Pseudocyst of pancreas

11. Serous cystadenoma (microcystic adenoma) (central stellate calcium)

12. Solid and papillary epithelial neoplasm

13. Teratoma

[ ] This condition does not actually cause the gamuted imaging finding, but can produce imaging changes that simulate it.

\section{References}

1. Burgener FA, Kormano M: Differential Diagnosis in Conventional Radiology. (ed 2) New York: Thieme Medical Publ, 1991, pp 538-540

2. Eisenberg RL: Gastrointestinal Radiology: A Pattern Approach. (ed 3) Philadelphia: Lippincott, 1996, pp 997-1002

3. Ring EJ, Eaton SB, Ferrucci JT, Short WF: Differential diagnosis of pancreatic calcification. AJR 1973;177:446-452

\section{Gamut G-220 \\ PANCREATIC LESION \\ CHARACTERIZED BY BLOOD OR HEMORRHAGE}

1. [Aneurysm of aorta, pancreaticoduodenal arcade, or splenic artery]

2. Hemangioma

3. Hemorrhagic pancreatitis

4. Hemorrhagic pseudocyst

5. Islet cell tumor

6. Metastasis (esp. from carcinoma of colon)

7. Serous cystadenoma (microcystic adenoma)

8. Sarcoma

9. Solid and papillary epithelial neoplasm

[ ] This condition does not actually cause the gamuted imaging finding, but can produce imaging changes that simulate it.

\section{Reference}

1. Amano Y, Oishi, Takahashi M, et al: Nonenhanced magnetic resonance imaging of mild acute pancreatitis. Abdom Imaging 2001;26:59-63

\section{Gamut G-221 \\ PANCREATIC LESION CHARACTERIZED BY FAT}

\section{UNCOMMON}

1. Focal fat deposit

2. Lipoma

3. Liposarcoma (possible in well-differentiated type)

4. Shwachman-Diamond S.

5. Teratoma

\section{Reference}

1. Itai Y, Saida Y, Kurosaki Y, et al: Focal fatty masses of the pancreas. Acta Radiol 1995;36:178-181 


\section{Gamut G-222}

\section{FATTY REPLACEMENT OR INFILTRATION OF PANCREAS}

\section{COMMON}

1. Aging

2. Cystic fibrosis (mucoviscidosis)

3. Obesity

4. Steroid therapy

\section{UNCOMMON}

1. Chronic pancreatitis

2. Diabetes mellitus

3. Hemochromatosis

4. Hereditary pancreatitis

5. Johanson-Blizzard S.

6. Lipomatous pseudohypertrophy

7. Malnutrition

8. Shwachman-Diamond S.

\section{References}

1. Barzilai M, Lerner A, Branski D: Increased reflectivity of the pancreas in rare hereditary pancreatic insufficiency syndromes. Clin Radiol 1996;51:575-576

2. Gore RM: Pancreas: Differential diagnosis. In: Gore RM, Levine MS: Textbook of Gastrointestinal Radiology. (ed 2) Philadelphia: WB Saunders, 2000, pp 1836-1843

3. Matsumoto S, Mori H, Miyake H, et al: Uneven fatty replacement of the pancreas: evaluation with CT. Radiology 1995; 194:453-458

4. Scafidi DE, Young LW: Diseases of the pediatric pancreas. In: Gore RM, Levine MS (eds): Textbook of Gastrointestinal Radiology. (ed 2) Philadelphia: WB Saunders, 2000, pp 2162-2173

5. Siegel MJ: Pediatric Body CT. Philadelphia: Lippincott Williams \& Wilkins, 1999

\section{Gamut G-223}

\section{HYPERVASCULAR PANCREATIC LESION (POSTCONTRAST CT, MRI, OR ANGIO)}

\section{COMMON}

1. Acute pancreatitis

2. Adenocarcinoma, pancreatic ductal (hypovascular but with neovascularity)

3. Aneurysm or pseudoaneurysm of hepatic, gastroduodenal, pancreaticoduodenal or splenic arteries

4. Islet cell tumor, incl. insulinoma; gastrinoma (esp. with Zollinger-Ellison S. or MEN S. Type I (tumor blush); glucagonoma; nonfunctioning islet cell tumor; somatostatinoma)

5. Metastasis, hypervascular (eg, from angiosarcoma; leiomyosarcoma; carcinoid; melanoma; renal cell carcinoma; carcinoma of adrenal or thyroid)

6. Mucinous cystadenoma or cystadenocarcinoma (macrocystic adenoma)

7. Serous cystadenoma (microcystic adenoma)

\section{UNCOMMON}

1. Abscess of pancreas

2. Castleman disease

3. Hemangioma

4. Intraductal papillary mucinous tumor of pancreas

5. Solid and papillary epithelial neoplasm (hypovascular but with enhanced solid tissue toward center of mass)

\section{References}

1. Adsay NV, Longnecker DS, Klimstra DS: Pancreatic tumors with cystic dilatation of the ducts: intraductal papillary mucinous neoplasms and intraductal oncocytic papillary neoplasms. Semin Diagn Pathol 2000;17:16-30

2. Gore RM: Pancreas: Differential diagnosis. In: Gore RM, Levine MS: Textbook of Gastrointestinal Radiology. (ed 2) Philadelphia: WB Saunders, 2000, pp 1836-1843

3. Irie $\mathrm{H}$, Honda $\mathrm{H}$, Kuroiwa $\mathrm{T}$, et al: Pitfalls in MR cholangiopancreatographic interpretation. RadioGraphics 2000; 24:229-234 
4. Larena JA, Astigarraga E, Saralegui I, et al: Magnetic resonance cholangiopancreatography in the evaluation of pancreatic duct pathology. Br J Radiol 1998;71:1100-1106

5. Scatarige JC, Horton KM, Sheth S, et al: Pancreatic parenchymal metastases: observations on helical CT. AJR 2001;176:695-701

\section{Gamut G-224}

\section{PANCREATIC ANGIOGRAPHIC ABNORMALITY (VIA CELIAC, SUPERIOR MESENTERIC, OR SUBSELECTIVE ARTERIOGRAPHY)}

\section{DISPLACEMENT OF VESSELS}

1. Gallbladder enlargement

2. Lymphoma

3. Pancreatic mass (See G-213)

\section{ENCASEMENT}

1. Irregular (esp. carcinoma of pancreas)

2. Long segment with arterial cuffing (esp. carcinoma)

3. Smooth (eg, carcinoma; pancreatitis)

\section{EXTRAVASATION}

1. Pancreatic abscess

2. Pancreatitis

\section{HYPERVASCULARITY (See G-223)}

1. Carcinoma

2. Cystadenoma; cystadenocarcinoma (florid neovascularity)

3. Islet cell tumor, esp. Zollinger-Ellison S.; MEN S. Type I (tumor blush)

4. Pancreatic abscess

5. Pancreatitis

\section{HYPOVASCULARITY}
1. Lymphoma ${ }_{g}$
2. Metastasis

3. Pancreatic pseudocyst

4. Pancreatitis

\section{OTHER}

1. Microaneurysms (esp. pancreatitis; polyarteritis nodosa)

2. Occlusion or truncation, arterial or venous (eg, carcinoma; surgical ligation; vascular disease)

\section{Gamut G-225 \\ DILATED PANCREATIC DUCT}

1. Aging

2. Calculus in distal common duct

3. Chronic pancreatitis

4. Pancreatic or ampullary mass (esp. carcinoma of pancreas or ampulla; focal acute pancreatitis)

\section{NOTE: NATURE OF DILATATION}

\section{Suggesting Pancreatitis}

1. Calculi in duct

2. Irregular dilatation of duct

\section{Suggesting Neoplasm}

1. Duct occupies $>50 \%$ of anteroposterior gland diameter

2. Smooth and beaded appearance of duct

\section{References}

1. Cheszmar JL: Pancreatic neoplasms. In: Gore RM, Levine MS (eds): Textbook of Gastrointestinal Radiology. (ed 2) Philadelphia: WB Saunders, 2000, pp 1796-1811

2. Gore RM: Pancreas: Differential Diagnosis. In Gore RM, Levine MS (eds): Textbook of Gastrointestinal Radiology. (ed 2) Philadelphia: WB Saunders, 2000, pp 1836-1843 


\section{Gamut G-226}

\section{PANCREATIC DUCT STRICTURE (ERCP, MRCP)}

\section{WITH NORMAL PANCREATIC PARENCHYMA}

1. Osteophyte of spine

2. Vascular compression (eg, aneurysm or pseudoaneurysm of aorta, gastroduodenal, pancreaticoduodenal, or splenic arteries)

\section{WITH ABNORMAL PARENCHYMA}

1. Carcinoma of pancreas

2. Chronic pancreatitis

3. Duct hyperplasia

\section{Reference}

1. Gore RM: Pancreas: Differential Diagnosis. In Gore RM, Levine MS (eds): Textbook of Gastrointestinal Radiology. (ed 2) Philadelphia: WB Saunders, 2000, pp 1836-1843.

\section{Gamut G-227}

\section{GAS IN PANCREATIC DUCT}

\section{COMMON}

1. Abscess

2. Patulous Vaterian sphincter

3. Post-endoscopic retrograde cholangiopancreatography (ERCP)

4. Prior Vaterian papillotomy

\section{UNCOMMON}

1. Duodenal diverticulum communication

2. Enteropancreatic fistula (spontaneous; surgical)

\section{Reference}

1. Gore RM: Pancreas: Differential diagnosis. In: Gore RM, Levine MS: Textbook of Gastrointestinal Radiology. (ed 2) Philadelphia: WB Saunders, 2000, pp 1836-1843

\section{Gamut G-228 \\ CYSTIC MESENTERIC OR INTRAPERITONEAL LESION (US, CT, MRI)}

\section{COMMON}

1. Abscess

2. Cystic lymphangioma

3. Hematoma

4. Mesenteric cyst

\section{UNCOMMON}

1. Ascites, complicated

2. Cystic mesothelioma

3. Cystic or necrotic stromal cell tumor ${ }_{g}$ (eg, leiomyoma; leiomyosarcoma)

4. Duplication cyst (enteric)

5. Hydatid disease

6. Lymphadenopathy, cystic or necrotic (eg, bacillary angiomatosis in AIDS)

7. Papillary serous carcinoma of peritoneum

8. Pseudocyst of pancreas invading mesentery

9. Pseudomyxoma peritonei

10. Teratoma

\section{References}

1. Chopra S, Laurie LR, Chintapalli KN, et al: Primary papillary serous carcinoma of the peritoneum: CT-pathologic correlation. J Comput Assist Tomogr 2000;24:395-399

2. Inman DS, Lambert AW, Wilkins DC: Multicystic peritoneal inclusion cysts: the use of CT guided drainage for symptom control. Ann R Coll Surg Eng 2000; 82:196-197

3. Ros P, Olmsted W, Moser R, Dachman A, et al: Mesenteric and omental cysts: Histologic classification with imaging correlation. Radiology 1987;164:327-332

4. Ros PR: Bubbles and marbles of the belly: cystic and solid masses of the mesentery and omentum. In: Balfe DM, Levine MS: RSNA Categorical Course in Diagnostic Radiology: Gastrointestinal. 1997, pp 59-66

5. Silverman PM, Cooper C: Mesenteric and omental lesions. In Gore RM, Levine MS (eds): Textbook of Gastrointestinal Radiology. (ed 2) Philadelphia: WB Saunders, 2000, pp 1980-1992 


\section{Gamut G-229}

\section{SOLID LESION OF THE MESENTERY OR MESENTERIC ROOT (US, CT, MRI)}

\section{COMMON}

1. Abscess $_{\mathrm{g}}$

2. Hematoma (trauma; bleeding disorder)

3. Lymphadenopathy (eg, metastatic carcinoma; tuberculosis; Crohn's disease; Castleman disease; AIDS-bacillary angiomatosis)

4. Lymphoma

5. Metastasis (esp. from colon or ovary)

6. Omental "cakes" (eg, metastatic disease; carcinoid; lymphoma; tuberculosis)

7. Stromal cell tumor ${ }_{\mathrm{g}}$ (esp. leiomyosarcoma; leiomyoma; neurofibroma)

\section{UNCOMMON}

1. Desmoid tumor (mesenteric fibromatosis); desmoplastic small round cell tumor

2. Hemangioma

3. Hydatid disease

4. Lipoma; lipomatosis; liposarcoma

5. Lymphangioma

6. Malignant fibrous histiocytoma

7. Mesothelioma

8. Phlegmon (pancreatitis); pseudocyst of pancreas

9. Retractile mesenteritis (chronic fibrosing mesenteritis; mesenteric lipodystrophy; panniculitis; WeberChristian disease)

10. Splenosis

11. Teratoma

\section{References}

1. Nicolas AI, Ros PR: Imaging of the mesentery and omentum. In: Grainger RG, Allison DJ: Grainger \& Allison's Diagnostic Radiology. (ed 3) New York: Churchill Livingstone, 1997, pp 1059-1079

2. Silverman PM, Cooper C: Mesenteric and omental lesions. In Gore RM, Levine MS (eds): Textbook of Gastrointestinal Radiology. (ed 2) Philadelphia: WB Saunders, 2000, pp 1980-1992

3. Slone RM, Fisher AJ: Pocket Guide to Body CT Differential Diagnosis. New York: McGraw-Hill, 1999, pp 191-192

\section{Gamut G-230}

\section{ALTERATION IN DENSITY OF THE MESENTERIC FAT ON CT ("Misty Mesentery")}

1. Hemorrhage (eg, trauma)

2. Inflammation (eg, Crohn's disease)

3. Lymphedema

4. Mesenteric edema

5. Neoplasm, eg, carcinomatosis; mesothelioma (esp. after chemotherapy)

6. Retractile mesenteritis (chronic fibrosing mesenteritis; mesenteric lipodystrophy; panniculitis; WeberChristian disease)

\section{References}

1. Mindelzun RE, Jeffrey RB Jr, Lane MJ, Silverman PM: The misty mesentery on CT: differential diagnosis. AJR 1996; 167:61-65

2. Silverman PM, Cooper C: Mesenteric and omental lesions. In Gore RM, Levine MS (eds): Textbook of Gastrointestinal Radiology. (ed 2) Philadelphia: WB Saunders, 2000, pp 1980-1992

\section{Gamut G-231 \\ PERITONEAL DISEASE}

\section{COMMON}

1. Abscess ${ }_{\mathrm{g}}$, abdominal or pericolic (See G-89)

2. Adhesions (eg, inflammatory; postoperative); congenital peritoneal bands (Ladd's bands)

3. Crohn's disease

4. Endometriosis

5. Fluid, ascites (See G-233, 234)

6. Lymphoma

7. Metastasis, implantation, or invasion by malignant neoplasm

8. Obesity

(continued) 
9. Pancreatitis (incl. saponification)

10. Peritonitis

11. Tuberculosis

\section{UNCOMMON}

1. Amyloidosis

2. Carcinoid

3. Cyst of mesentery

4. Desmoid tumor (mesenteric fibromatosis), isolated or with Gardner S.

5. Fungus disease (eg, actinomycosis) $_{\mathrm{g}}$

6. Medication (eg, practolol peritonitis; Sansert)

7. Mesothelioma

8. Parasitic disease (esp. hydatid disease; amebiasis; pentastomiasis-Armillifer infection)

9. Pseudomyxoma peritonei

10. Radiation peritonitis

11. Retractile mesenteritis (chronic fibrosing mesenteritis; mesenteric lipodystrophy; panniculitis; WeberChristian disease)

12. Sarcoma

13. Typhoid fever

\section{References}

1. Banner MP, Gohel VK: Peritoneal mesothelioma. Radiology 1978; 129:637-640

2. Sacks B, Joffe N, Harris N: Isolated mesenteric desmoids (mesenteric fibromatosis). Clin Radiol 1978; 29:95-100

3. Silverman PM, Cooper C: Mesenteric and omental lesions. In Gore RM, Levine MS (eds): Textbook of Gastrointestinal Radiology. (ed 2) Philadelphia: WB Saunders, 2000, pp 1980-1992

\section{Gamut G-232}

\section{DIFFUSE PERITONEAL THICKENING} (US, CT, MRI)

\section{COMMON}

1. Carcinomatosis

2. Peritonitis

3. Postoperative state

\section{UNCOMMON}

1. Lymphoma

2. Mesothelioma

3. Pseudomyxoma peritonei

4. Sarcomatosis

5. Tuberculosis

\section{Reference}

1. Silverman PM, Cooper C: Mesenteric and omental lesions. In Gore RM, Levine MS (eds): Textbook of Gastrointestinal Radiology. (ed 2) Philadelphia: WB Saunders, 2000, pp 1980-1992

\section{Gamut G-233}

\section{PERITONEAL FLUID COLLECTION (ASCITES) IN AN ADULT (ESP. ON US, CT, MRI)}

\section{INFECTION OR INFLAMMATION OF THE PERITONEAL CAVITY (PERITONITIS)}

1. Abscess

2. Pancreatitis

3. Pelvic inflammatory disease (PID)

4. Rupture of a hollow viscus (eg, appendicitis; diverticulitis; empyema of gallbladder; peptic ulcer; typhoid fever)

5. Tuberculosis or other primary infection causing peritonitis 


\section{LYMPHATIC OBSTRUCTION WITH CHYLOUS OR LYMPH ASCITES (See G-235)}

\section{Congenital}

1. Milroy disease

2. Tuberous sclerosis

\section{Elevated lymphatic pressure}

1. Cirrhosis

2. Constrictive pericarditis

3. Heart failure

\section{Infection}

1. Filariasis

\section{Neoplastic disease}

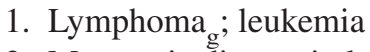

2. Metastatic disease in lymphatics

\section{Radiation therapy}

\section{Trauma}

1. Rupture of abdominal lymphatics

\section{NEOPLASM}

1. Benign (eg, Meigs S. - benign ovarian tumor)

2. Malignant (eg, carcinoma of gastrointestinal tract or ovary with mesenteric or peritoneal metastases; mesothelioma)

\section{VENOUS OBSTRUCTION}

\section{Cardiac disease with chronic elevation of venous pressure}

1. Chronic right heart failure due to other causes

2. Constrictive pericarditis

3. Mitral stenosis

\section{External pressure on the portal vein}

1. Inflammatory lymph nodes (eg, tuberculosis)

2. Lymphoma

3. Malignant tumor, primary or metastatic

4. Sarcoidosis
Obstruction of the inferior vena cava above the hepatic vein

1. Mediastinal mass

2. Thrombosis of IVC

\section{Portal vein obstruction secondary} to diffuse hepatic disease

1. Hepar lobatum

2. Portal cirrhosis

3. Schistosomiasis

\section{Portal vein thrombosis}

1. Chronically ill patients

2. Invasion by neoplastic disease

\section{Veno-occlusive disease}

Thrombosis or web in inferior vena cava

\section{OTHER CAUSES}

1. Bile peritonitis

2. Ectopic pregnancy

3. Hemoperitoneum (eg, trauma; surgery)

4. Hypoalbuminemia (eg, nephrotic S.; protein-losing gastroenteropathy)

5. Peritoneal lavage; dialysis

\section{[CONDITIONS THAT MAY MIMIC ASCITES]}

1. [Mesenteric cyst]

2. [Ovarian cyst]

3. [Pancreatic pseudocyst]

4. [Pregnancy]

\section{References}

1. Gore RM, Gore MD: Ascites and peritoneal fluid collections. In Gore RM, Levine MS (eds): Textbook of Gastrointestinal Radiology. (ed 2) Philadelphia: WB Saunders, 2000, pp 1969-1979

2. Williamson MR: Chapter 5. Abdominal Ultrasound. In: Essentials of Ultrasound. Philadelphia: WB Saunders, 1996, p 66 


\section{Gamut G-234}

\section{PERITONEAL FLUID (ASCITES) IN AN INFANT OR CHILD}

\section{COMMON}

1. Appendiceal perforation

2. Cardiac disease (eg, anasarca; constrictive pericarditis)

3. Cirrhosis, portal or biliary (eg, biliary atresia; polycystic liver)

4. Hemorrhage

5. Hydrops fetalis; erythroblastosis fetalis

6. Hypoproteinemia (eg, malnutrition; kwashiorkor; intestinal lymphangiectasia)

7. Peritonitis (eg, meconium; sepsis; toxoplasmosis; rubella S.; cytomegalovirus infection; typhoid fever; amebiasis)

8. Portal vein or IVC obstruction, extrahepatic (eg, neoplasm; lymphadenopathy; thromboembolism)

9. Renal failure (eg, nephrotic S. with hypoproteinemia; glomerulonephritis; uremia)

10. Trauma; surgery

11. Urinary outlet obstruction with hydronephrosis (urine ascites)

12. Ventriculoperitoneal shunt

\section{UNCOMMON}

1. Antitrypsinase deficiency

2. Chylous or lymphatic ascites, neonatal (See G-235)

3. Galactosemia; tyrosinemia

4. Gaucher disease; lysosomal storage disease

5. Hemolytic anemia with ascites

6. Hydrometrocolpos with rupture and plastic peritonitis

7. Meckel diverticulum, perforated

8. Meigs S. (benign ovarian tumor)

9. Metastatic disease to peritoneum

10. Ovarian cyst, ruptured

11. Pancreatitis, acute

12. Preduodenal portal vein

13. Schistosomiasis
14. Thromboembolism of inferior vena cava, renal vein, or hepatic veins

15. Tuberculosis

16. Wilson disease

\section{References}

1. Meyer JI, Donaldson JS: Diseases of the pediatric abdominal wall, peritoneum, and mesentery. In Gore RM, Levine MS (eds): Textbook of Gastrointestinal Radiology. (ed 2) Philadelphia: WB Saunders, 2000, pp 2179-2185

2. Moncada R, Wang JJ, Love L, Bush I: Neonatal ascites associated with urinary outlet obstruction (urine ascites). Radiology 1968; 90:1165-1170

3. Siegel MJ: Pediatric Body CT. Philadelphia: Lippincott Williams \& Wilkins, 1999

4. Silverman FN (ed): Caffey's Pediatric X-ray Diagnosis: An Integrated Imaging Approach. (ed 8) Chicago: Year Book Medical Publ, 1985

5. Swischuk LE: Radiology of the Newborn, Infant, and Young Child. (ed 3) Baltimore: Williams \& Wilkins, 1989

6. Swischuk LE, John SD: Differential Diagnosis in Pediatric Radiology. (ed 2) Baltimore: Williams \& Wilkins, 1995, pp 160-16

\section{Gamut G-235}

\section{CHYLOUS OR LYMPHATIC ASCITES*}

\section{COMMON}

1. Filariasis

2. Lymphoma

3. Metastatic disease (esp. to lymph nodes)

4. Neoplasm, benign (esp. lymphangioma)

5. Neoplasm, malignant, compressing or invading lymphatic system (eg, carcinoma of pancreas, lung, esopaghus; mediastinal tumor)

6. Postoperative

7. Trauma

\section{UNCOMMON}

1. Adhesive bands

2. Cirrhosis (hepatic lymphatics visible)

3. Congenital absence or hypoplasia of lymphatic system 
4. Idiopathic

5. Lymphangioleiomyomatosis; tuberous sclerosis

6. Lymphangiomatosis, disseminated (benign metastasizing lymphangiomatosis)

7. Tuberculosis; histoplasmosis

* Obstruction of abdominal lymphatic vessels, cisterna chyli, or thoracic duct on lymphangiography.

\section{References}

1. Gore RM, Gore MD: Ascites and peritoneal fluid collections. In Gore RM, Levine MS (eds): Textbook of Gastrointestinal Radiology. (ed 2) Philadelphia: WB Saunders, 2000, pp 1969-1979

2. Griscom NT, Colodny AH, Rosenberg HK, et al: Diagnostic aspects of neonatal ascites: Report of 27 cases. AJR 1977;128:961-970

\section{Gamut G-236}

\section{SPONTANEOUS PNEUMOPERITONEUM WITHOUT PERITONITIS}

\section{COMMON}

1. Iatrogenic (incl. postoperative; anesthesia; respiratory therapy; peritoneal dialysis; Rubin test; bronchoscopy; endoscopy; diagnostic pneumoperitoneum)

2. Idiopathic

3. Perforation of GI tract, forme fruste type (eg, peptic ulcer; neoplasm; Crohn's disease; bowel infarction or obstruction)

4. Vaginal "aspiration" (eg, douching; sudden squatting; oral sex; postpartum exercises)

\section{UNCOMMON}

\section{ARDS}

2. Distention of stomach (eg, aerophagia; gastroscopy; sodium bicarbonate ingestion; misplaced oxygen tube)

3. Idiopathic dilatation of colon (eg, pseudo-obstruction)

4. Jejunal diverticulosis
5. Pneumatosis cystoides intestinalis (See G-70)

6. Pneumomediastinum (eg, pulmonary emphysema; ruptured bulla)

7. Pneumothorax (eg, via congenital pleuroperitoneal fistula)

8. Sclerotherapy of esophageal varices

\section{References}

1. Ashai S, Lipton D, Colon A, et al: Pneumoperitoneum secondary to cunnilingus. N Engl J Med 1976; 295:117

2. Baker SR, Cho KC: The Abdominal Plain Film with Correlative Imaging. Norwalk, CT: Appleton \& Lange, 1998

3. Campbell RE, Boggs TR Jr, Kirkpatrick JA Jr: Early neonatal pneumoperitoneum from progressive massive tension pneumomediastinum. Radiology 1975;114:121-126

4. Eisenberg RL: Gastrointestinal Radiology: A Pattern Approach. (ed 3) Philadelphia: Lippincott, 1996, pp 939-949

5. Felson B, Wiot JF: Another look at pneumoperitoneum. Semin Roentgenol 1973; 8:437-443

6. Stringfield JT III, Graham JP, Watts CM, et al: Pneumoperitoneum: a complication of mechanical ventilation. JAMA 1976;235:744-746

\section{Gamut G-237 \\ PNEUMOPERITONEUM WITH PERITONITIS}

\section{COMMON}

1. Perforated hollow viscus (eg, peptic ulcer; intestinal obstruction; diverticulitis; appendicitis; necrotic tumor; penetrating or blunt abdominal trauma; emphysematous cholecystitis) (See G-238)

\section{UNCOMMON}

1. Meconium peritonitis

2. Megacolon with rupture (eg, Chagas' disease; Hirschsprung disease)

3. Perforation of colon following immunosuppressive therapy in renal transplant patient

4. Septic peritonitis with gas forming organism

(continued) 
5. Ulcerating bowel disease (eg, typhoid fever; amebiasis; toxic megacolon; ulcerative colitis; Crohn's disease; tuberculosis; pseudomembranous colitis; necrotizing enterocolitis; ischemic colitis; lymphogranuloma venereum)

\section{References}

1. Baker SR, Cho KC: The Abdominal Plain Film with Correlative Imaging. Norwalk, CT: Appleton \& Lange, 1998

2. Eisenberg RL: Gastrointestinal Radiology: A Pattern Approach. (ed 3) Philadelphia: Lippincott, 1996, pp 939-946

\section{Gamut G-238 \\ PERFORATED HOLLOW VISCUS IN AN INFANT}

\section{COMMON}

1. Iatrogenic (eg, intubation; rectal thermometer; enema; resuscitation; intrauterine transfusion)

2. Intestinal obstruction (eg, atresia; volvulus)

3. Meconium ileus (cystic fibrosis $\{$ mucoviscidosis $\}$ )

4. Necrotizing enterocolitis

5. Respirator therapy

6. Trauma; battered child S.

\section{UNCOMMON}

1. Gastric rupture of newborn

2. Hirschsprung disease

3. Idiopathic

4. Ischemic enteritis (eg, umbilical artery thromboembolism; sepsis; malrotation with midgut volvulus)

5. Meconium plug $\mathrm{S}$.

6. Perforated peptic or stress ulcer

7. [Pneumoperitoneum without peritonitis] (See G-236)

8. Small left colon $\mathrm{S}$.

\section{References}

1. Fernbach SK: Neonatal gastrointestinal radiology. In: Gore RM, Levine MS (eds): Textbook of Gastrointestinal Radiology. (ed 2) Philadelphia: WB Saunders, 2000, pp 20422073
2. Leonidas JC, Hall RT, Rhodes PG, et al: Pneumoperitoneum in ventilated newborns. A medical or a surgical problem? Am J Dis Child 1974;128:677-6803.

3. Tucker AS, Soine L, Izant Jr RJ: Gastrointestinal perforations in infancy: Anatomic and etiologic gamuts. AJR 1975; 123:755-763

\section{Gamut G-239}

\section{ABNORMAL GAS COLLECTION IN THE RIGHT UPPER QUADRANT}

\section{COMMON}

1. Abscess ${ }_{\mathrm{g}}$, abdominal (eg, subphrenic, hepatic, pericolic, renal, or perirenal) (See G-243)

2. Colon interposition (Chilaiditi S.); colon distention

3. Gas in the biliary tract; biliary fistula (See G-128, G-139)

4. Pneumoperitoneum, free or loculated (eg, perforation of hollow viscus; postoperative) (See G-236-238)

5. Subhepatic gas or abscess (eg, perforated duodenal ulcer or appendix)

\section{UNCOMMON}

1. Abdominal wall gas or abscess (postoperative; traumatic; drainage tube)

2. Emphysematous cholecystitis

3. Gastric rupture of newborn

4. Iatrogenic pneumoretroperitoneum (incl. interventional embolization of a viscus; needle or catheter drainage; postnephrectomy; endoscopy)

5. Pneumatosis intestinalis (See G-70)

6. Pneumomediastinum with retroperitoneal extension

7. Portal vein gas (See G-192)

8. Retroperitoneal rupture of gut (esp. duodenum or rectum)

9. Stomach (heterotaxy)

\section{References}

1. Anschuetz SL: Extraluminal gas in the upper abdomen. Semin Roentgenol 1984;19:255 
2. Baker SR, Cho KC: The Abdominal Plain Film with Correlative Imaging. Norwalk, CT: Appleton \& Lange, 1998

3. Eisenberg RL: Gastrointestinal Radiology: A Pattern Approach. (ed 3) Philadelphia: Lippincott, 1996, pp 950-964

4. Love L, Baker D, Ramey R: Gas producing perinephric abscess. AJR 1973;119:783-7925.

5. Rice RP, Thompson WM, Gedgaudas RK: The diagnosis and significance of extraluminal gas in the abdomen. Radiol Clin North Am 1982;20:819-837

\section{Gamut G-240}

\section{ABNORMAL GAS COLLECTION IN THE LEFT UPPER QUADRANT}

\section{COMMON}

1. Abscess ${ }_{\mathrm{g}}$, other abdominal (eg, subphrenic, splenic, pericolic, renal or perirenal)

2. Pneumoperitoneum, free or loculated (eg, perforation of hollow viscus; postoperative) (See G-236-238)

\section{UNCOMMON}

1. Abdominal wall gas or abscess (postoperative; traumatic; drainage tube)

2. Emphysematous gastritis

3. Iatrogenic pneumoretroperitoneum (incl. interventional embolization of a viscus; needle or catheter drainage; postnephrectomy; endoscopy)

4. Lesser sac gas or abscess (eg, postsplenectomy; perforation of gut; pancreatic abscess or infected pseudocyst)

5. Pancreatic gas abscess (peritoneal fat necrosis)

6. Pneumatosis intestinalis

7. Pneumomediastinum with retroperitoneal extension

8. Retroperitoneal rupture of gut (esp. duodenum or rectum)

\section{References}

1. Anschuetz SL: Extraluminal gas in the upper abdomen. Semin Roentgenol 1984;19:255

2. Baker SR, Cho KC: The Abdominal Plain Film with Correlative Imaging. Norwalk, CT: Appleton \& Lange, 1983.
3. Eisenberg RL: Gastrointestinal Radiology: A Pattern Approach. (ed 3) Philadelphia: Lippincott, 1996, pp 950-964.

4. Love L, Baker D, Ramey R: Gas producing perinephric abscess. AJR 1973;119:783-792

\section{Gamut G-241}

\section{LARGE ABDOMINAL GAS POCKET}

\section{COMMON}

1. Abscess $_{\mathrm{g}}$, abdominal (eg, subphrenic, splenic, renal or perirenal, pericolic, pancreatic, lesser sac, abdominal wall)

2. Bladder distended with air (eg, iatrogenic; emphysematous cystitis)

3. Cecal distention (eg, nonobstructive "paralytic" ileus; pseudo-obstruction; colon obstruction)

4. Diabetic gastropathy; acute gastric dilatation (eg, cast S.)

5. Duodenal obstruction (See G-42, 43)

6. Gastric outlet obstruction (See G-30)

7. Hernia, obstructed (eg, hiatal; diaphragmatic; internal; external)

8. Pneumoperitoneum, free or loculated (subphrenic; lesser sac; greater sac)

9. Retroperitoneal or extraperitoneal gas (eg, duodenal or rectal perforation)

10. Sentinel loop (localized ileus) (See G-80)

11. Small bowel or colon obstruction, chronic (eg, Crohn's disease; adhesions; congenital stenosis)

\section{UNCOMMON}

1. Abdominal wall gas (postoperative)

2. Amnionitis; endometritis (physometra); infected fetus

3. Blind loop S. (esp. postoperative) (See G-58)

4. Diverticulum of colon or duodenum (giant size)

5. Duplication cyst, communicating

6. Emphysematous cholecystitis

7. Emphysematous peritonitis

8. Fibroid, infected 
9. Gangrene of liver

10. Hydropneumometrocolpos; vaginitis emphysematosa

11. Meckel's diverticulum (giant size)

12. Ovarian cyst (eg, gas infection; intestinal fistula)

\section{References}

1. Altemeier WA, Culbertson WR, Fullen WD, et al: Intra-abdominal abscesses. Am J Surg 1973;125:70-79

2. Blatt ES, Schneider HJ, Wiot JF, et al: Roentgen findings in obstructed diaphragmatic hernia. Radiology 1962;79:6486573.

3. Felson B, Wiot JF: Case of the Day. Springfield, IL: CC Thomas, 1967, p 764.

4. Gaillard S, Pascaud E, Chardac J, et al: An unusual cause of intra-abdominal gas pouches. Ann Radiol 1980;23:6756785.

5. Messmer JM: Gas and soft tissue abnormalities. In Gore RM, Levine MS (eds): Textbook of Gastrointestinal Radiology. (ed 2) Philadelphia: WB Saunders, 2000, pp 157-177

6. Phillips JC: A spectrum of radiologic abnormalities due to tubo-ovarian abscess. Radiology 1974;110:307-311

\section{Gamut G-242}

\section{ABDOMINAL OR PELVIC ABSCESS}

\section{COMMON}

1. Appendicitis

2. Carcinoma or other malignancy with perforation

3. Crohn's disease

4. Diverticultis

5. Pancreatitis

6. Pelvic inflammatory disease (eg, endometritis)

7. Peptic ulcer perforation

8. Postoperative

9. Posttraumatic (eg, gunshot or knife wound)

10. Tubo-ovarian (eg, sexually transmitted diseases; ectopic pregnancy)

\section{UNCOMMON}

1. Intestinal perforation, other (eg, intestinal obstruction; meconium ileus)

2. Ischemic colitis

3. Lymphogranuloma venereum

4. Parasitic disease (eg, amebiasis; schistosomiasis; ascariasis)

5. Perinephric abscess; pyelonephritis

6. Tuberculosis

7. Typhoid fever

\section{Gamut G-243}

\section{RIGHT ANTERIOR PARARENAL SPACE ABSCESS (ESPECIALLY ON US, CT, OR MRI)}

1. Duodenal perforation secondary to
a. Ulcer
b. Foreign body
c. Inflamed diverticulum of duodenum

2. Crohn's disease of small bowel or ascending colon

3. Colitis involving ascending colon
a. Amebiasis
b. Diverticulitis
c. Tuberculosis

4. Ruptured retrocecal or retroperitoneal appendix

5. Pancreatic abscess or infected pseudocyst

6. Renal or perirenal abscess extending into pararenal space

\section{Reference}

1. Meyers MA: Dynamic Radiology of the Abdomen (ed 5) New York: Springer-Verlag, 2000 


\section{Gamut G-244-S}

\section{ABSCESS MIMICS ON ABDOMINAL US, CT, OR MRI}

\section{COMMON}

1. Cyst (eg, ovarian; renal; splenic; dermoid; mesenteric; hepatic; hydatid)

2. Hematoma

3. Loop of bowel

4. Necrotic neoplasm (eg, hepatoma; metastasis)

5. Pseudocyst of pancreas

\section{UNCOMMON}

1. Biloma

2. Iatrogenic (eg, retained Foley catheter)

3. Herniation of bowel through diaphragm

4. Lymphocele

5. Seroma

6. Urinoma

\section{Gamut G-245}

\section{DECREASED ABDOMINAL GAS IN A NEWBORN}

\section{COMMON}

1. Congenital diaphragmatic hernia

2. Duodenal atresia or stenosis; annular pancreas

3. Endotracheal intubation

4. Esophageal obstruction (eg, atresia without T-E fistula; web) (See G-12, G-19)

5. Medication (esp. maternal)

6. Neonatal sepsis

7. Obstruction of pylorus (eg, hypertrophic pyloric stenosis at 2 to 8 weeks), duodenum, or proximal jejunum (esp. atresia or stenosis)

8. Suction, orogastric or nasogastric

\section{UNCOMMON}

1. Dehydration

2. Dysphagia; impaired swallowing physiology (eg, severe prematurity; depressed swallowing reflex; impending death)

3. Fluid-filled bowel

4. Large abdominal mass

5. Midgut volvulus

6. Normal

7. Peritoneal fluid (ascites)

8. Vomiting (eg, gastroenteritis; electrolyte imbalance)

\section{References}

1. Cohen MD, Jansen R, Lemons J, et al: Evaluation of the gasless abdomen in the newborn and young infant with metrizamide. AJR 1984;142:393-396

2. Fernbach SK: Neonatal gastrointestinal radiology. In Gore RM, Levine MS (eds): Textbook of Gastrointestinal Radiology. (ed 2) Philadelphia: WB Saunders, 2000, pp 20422073

\section{Gamut G-246}

\section{DECREASED ABDOMINAL GAS IN AN ADULT}

\section{COMMON}

1. Acute pancreatitis

2. Ascites

3. Normal

\section{UNCOMMON}

1. Dysphagia

2. Fluid-filled bowel (eg, closed loop obstruction)

3. Hernia (esp. hiatal)

4. Large abdominal mass displacing bowel laterally

5. Mesenteric infarction, early

6. Obstruction of esophagus (eg, cardiospasm; neoplasm), stomach (eg, volvulus; pyloric obstruction from ulcer or neoplasm), or duodenum (eg, neoplasm)

7. Vomiting; inanition 


\section{Gamut G-247-1}

\section{ABDOMINAL CALCIFICATION(S) IN AN INFANT OR CHILD (See G-143, G-203, 204, G-247-2)}

\section{COMMON}

1. Adrenal calcification (See H-123)

2. Appendiceal fecalith (calculus)

3. [Foreign material; pills; pica; medication; contrast medium; heavy metal]

4. Histoplasmosis or tuberculosis in liver, spleen, lymph node, peritoneum

5. Meconium in peritoneum (meconium peritonitis from intestinal perforation), intestinal lumen or wall

6. Neuroblastoma; other neurogenic neoplasm

7. Urinary tract calcification (See H-24)

\section{UNCOMMON}

1. Abscess $_{\mathrm{g}}$, abdominal (esp. liver)

2. Arterial calcification (eg, metastatic calcinosis; primary or secondary hyperparathyroidism)

3. Calculus in Meckel's diverticulum or urachal cyst

4. Chronic granulomatous disease of childhood

5. Congenital syndromes (See G-247-2)

6. Cyst of spleen, kidney, pancreas, ovary, mesentery

7. Dermoid cyst; teratoma; fetus in fetu

8. Duplication, atresia, other obstruction of gut (mural or luminal calcification)

9. Enterolith; also enterolithiasis associated with anal atresia

10. Fetal infection (toxoplasmosis; rubella; cytomegalovirus; herpes simplex; varicella)

11. Gallstone (esp. thalassemia; sickle cell disease; other hemolytic anemia)

12. Hemangioma; lymphangioma (phlebolith; lympholith)

13. Hematoma, old (liver; spleen; retroperitoneal)

14. Hemochromatosis of liver

15. Hydrometrocolpos (ruptured) with plastic peritonitis

16. Ischemic infarct or necrosis of liver
17. Liver neoplasm (hemangioma; hamartoma; metastatic neuroblastoma; occasionally hepatoma; hepatoblastoma) (See G-154, 155)

18. Mucocele of appendix

19. Pancreatitis, chronic

20. Parasitic disease (eg, hydatid cysts or Armillifer infestation of peritoneum or mesentery; guinea worm infection or cysticercosis of abdominal wall or back)

21. Retroperitoneal neoplasm

22. Thromboembolism of inferior vena cava, portal vein, renal vein

23. Tuberculous psoas abscess

[ ] This condition does not actually cause the gamuted imaging finding, but can produce imaging changes that simulate it.

\section{References}

1. Baker SR, Cho KC: The Abdominal Plain Film with Correlative Imaging. Norwalk, CT: Appleton \& Lange, 1998

2. Berdon WE, Baker DH, Wigger HJ, et al: Calcified intraluminal meconium in newborn males with imperforate anus: enterolithiasis in the newborn. AJR 1975;125:449-455

3. Friedman AP, Haller JO, Boyer B, Cooper R: Calcified portal vein thromboemboli in infants: Radiography and ultrasonography. Radiology 1981;140:381-382

4. Nguyen DL, Leonard JC: Ischemic hepatic necrosis: A cause of fetal liver calcification. AJR 1986;147:596-597

5. Schackelford GD, Kirks DR: Neonatal hepatic calcification secondary to transplacental infection. Radiology 1977;122: 753-757

6. Siegel MJ: Pediatric Body CT. Philadelphia: Lippincott Williams \& Wilkins, 1999

7. Swischuk LE, John SD: Differential Diagnosis in Pediatric Radiology. (ed 2) Baltimore: Williams \& Wilkins, 1995

\section{Gamut G-247-2}

\section{CONGENITAL SYNDROMES WITH ABDOMINAL CALCIFICATIONS}

1. Beckwith-Wiedemann S. (adrenal)

2. Cholesterol ester storage disease (adrenal)

3. Chondroectodermal dysplasia (Ellis-van Creveld S.) (gallstones; nephrocalcinosis)

4. Chronic granulomatous disease of childhood (liver; spleen; lymph nodes) 
5. Cushing S. (adrenal)

6. Cystic fibrosis (mucoviscidosis) (pancreas; meconium peritonitis)

7. Cystinosis (urinary calculi)

8. Fetal cytomegalovirus infection (gonads)

9. Fetal herpes simplex infection (liver; adrenal)

10. Fetal toxoplasmosis infection (liver)

11. Fetal varicella infection (liver)

12. Gorlin S. (nevoid basal cell carcinoma S.) (ovarian)

13. Hirschsprung disease (enterolith)

14. Milk-alkali S. (nephrocalcinosis)

15. Multiple endocrine neoplasia (MEN) S., type IIA and IIB (eg, pheochromocytoma) (See J-5)

16. Oxalosis (nephrocalcinosis)

17. Prune-belly S. (Eagle-Barrett S.) (bladder wall; renal)

18. Renal tubular acidosis S. (nephrocalcinosis)

19. Wolman disease (familial xanthomatosis) (adrenal)

\section{References}

1. Kirks DR, Taybi H: Prune belly syndrome: an unusual cause of neonatal abdominal calcification. AJR 1975;123:7787822.

2. Swischuk LE, John SD: Differential Diagnosis in Pediatric Radiology. (ed 2) Baltimore: Williams \& Wilkins, 1995

3. Taybi H, Lachman RS: Radiology of Syndromes, Metabolic Disorders, and Skeletal Dysplasias. (ed 4) St. Louis: MosbyYear Book, 1996, p 958

\section{Gamut G-248}

\section{NONVISCERAL ABDOMINAL CALCIFICATION}

\section{COMMON}

1. Aneurysm; arteriovenous malformation

*2. Appendiceal fecalith (calculus), extruded into peritoneal sac

3. Atherosclerosis

*4. Dermoid cyst; teratoma; fetus in fetu

*5. [Foreign material (eg, pill; pica; medication; contrast medium; heavy metal)]
*6. Gallstone extruded into peritoneal sac

*7. Lymph nodes (eg, tuberculosis; histoplasmosis)

*8. Meconium in peritoneum, intestinal wall or lumen

*9. Phlebolith; lympholith (incl. hemangioma; lymphangioma)

*10. [Rib cartilage]

\section{UNCOMMON}

*1. [Barium in peritoneum]

2. [Bone lesion with matrix or sclerosis]

*3. Epiploic appendage

4. Fluorosis (ligamentous)

5. Hydrometrocolpos

6. Lipoma

*7. Lithopedion; extrauterine pregnancy

*8. Mesenteric cyst

9. Mesothelioma of peritoneum

10. Metastasis (esp. from colloid carcinoma; papillary cystadenocarcinoma of ovary)

*11. Mineral oil granuloma (instilled in peritoneum)

12. Myositis (fibrodysplasia) ossificans progressiva

13. Neoplasm of soft tissues (eg, osteoma; osteosarcoma; chondrosarcoma; undifferentiated abdominal malignancy)

14. Neuroblastoma

15. Pancreatitis with saponification; fat necrosis; Weber-Christian disease

*16. Parasitic disease (eg, hydatid cysts or Armillifer infestation of peritoneum or mesentery; guinea worm infection or cysticercosis of abdominal wall or back)

17. Pheochromocytoma

18. Pseudomyxoma peritonei (eg, from pseudomucinous cystadenoma of ovary; mucocele of appendix)

19. Retroperitoneal hematoma

20. Retroperitoneal neoplasm

21. Scar or burn (abdominal wall)

22. Scleroderma; dermatomyositis

23. Thrombosis of portal vein, renal vein, or inferior vena cava

24. Tuberculous peritonitis, psoas abscess

* May be mobile.

[ ] This condition does not actually cause the gamuted imaging finding, but can produce imaging changes that simulate it. 


\section{References}

1. Baker SR, Cho KC: The Abdominal Plain Film with Correlative Imaging. Norwalk, CT: Appleton \& Lange, 1998

2. Eisenberg RL: Gastrointestinal Radiology: A Pattern Approach. (ed 3) Philadelphia: Lippincott, 1996, pp 10431064

\section{Gamut G-249}

\section{ABDOMINAL CALCIFICATIONS THAT LAYER IN THE UPRIGHT POSITION}

\section{COMMON}

1. Bladder calculi

2. Gallstones

\section{UNCOMMON}

1. [Contrast medium; barium]

2. Dermoid cyst (eg, teeth)

3. Enteroliths in small bowel or Meckel's diverticulum

4. Milk of calcium (eg, in gallbladder or kidney, Pott's abscess, meconium, granulomatous lymph node, or chronic tubo-ovarian abscess)

5. Myxoglobulosis of appendix

6. Renal calculi (eg, in calyceal diverticulum or hydronephrosis)

\section{Urachal cyst calculi}

[ ] This condition does not actually cause the gamuted imaging finding, but can produce imaging changes that simulate it.

\section{Reference}

1. Baker SR, Cho KC: The Abdominal Plain Film with Correlative Imaging. Norwalk, CT: Appleton \& Lange, 1998

\section{Gamut G-250}

\section{ABDOMINAL CALCIFICATION(S) WITH A CONCRETION OR ANNULAR MORPHOLOGY}

\section{COMMON}

1. Aneurysm; arteriovenous malformation; atherosclerosis

2. Appendiceal fecalith (calculus)

3. [Foreign body (eg, pill)]

4. Gallstone

5. Meconium

6. Pancreatic calculus

7. Phlebolith or lympholith (eg, normal pelvic veins; splenolith or hepatolith; hemangioma or lymphangioma; varicocele)

8. Prostatic calculus

9. Urinary tract calculus

10. Varix

\section{UNCOMMON}

1. Cyst (mesenteric; ovarian; renal; splenic)

2. Dermoid cyst

3. Diverticulum calculus (eg, colonic; duodenal; Meckel's)

4. Enterolith

5. Epiploic appendage

6. Lithopedion

7. Mineral oil granuloma

8. Mucocele or myxoglobulosis of appendix

9. Parasitic disease (esp. hydatid disease-Echinococcus granulosus or E. multilocularis; Armillifer infection; guinea worm infection or cysticercosis of abdominal wall or back)

10. Pseudomyxoma peritonei (eg, from pseudomucinous cystadenoma of ovary; mucocele of appendix)

11. Urachal calculus

12. Urethral calculus

[ ] This condition does not actually cause the gamuted imaging finding, but can produce imaging changes that simulate it. 


\section{Reference}

1. Baker SR, Cho KC: The Abdominal Plain Film with Correlative Imaging. Norwalk, CT: Appleton \& Lange, 1998

\section{Gamut G-251}

\section{WIDESPREAD ABDOMINAL CALCIFICATIONS}

\section{COMMON}

1. Atherosclerosis

2. Phleboliths (eg, normal; hemangioma)

3. Tuberculosis or histoplasmosis in liver, spleen, lymph nodes, or peritoneum

\section{UNCOMMON}

1. Fat necrosis (esp. pancreatitis; Weber-Christian disease)

2. [Hemochromatosis (liver and spleen)]

3. Meconium peritonitis

4. Metastases (eg, neuroblastoma; ovarian cystadenocarcinoma; colloid carcinoma of colon)

5. Mineral oil granulomas (peritoneal instillation)

6. Myositis (fibrodysplasia) ossificans progressiva (esp. abdominal wall)

7. Parasitic disease (esp. hydatid cysts or Armillifer infestation of peritoneum or mesentery; guinea worm infection or cysticercosis of abdominal wall or back)

8. Pseudomyxoma peritonei (eg, from pseudomucinous cystadenoma of ovary; mucocele of appendix)

9. Scleroderma; dermatomyositis (abdominal wall)

10. Undifferentiated abdominal malignancy

[ ] This condition does not actually cause the gamuted imaging finding, but can produce imaging changes that simulate it.

\section{References}

1. Baker SR, Cho KC: The Abdominal Plain Film with Correlative Imaging. Norwalk, CT: Appleton \& Lange, 1998

2. Eisenberg RL: Gastrointestinal Radiology: A Pattern Approach. (ed 3) Philadelphia: Lippincott, 1996, pp 1044-1049

3. Swischuk LE, John SD: Differential Diagnosis in Pediatric Radiology. (ed 2) Baltimore: Williams \& Wilkins, 1995

\section{Gamut G-252}

\section{ABDOMINAL WALL CALCIFICATION}

1. Fat necrosis

2. Hypercalcemic state; idiopathic calcinosis

3. Myositis (fibrodysplasia) ossificans progressiva; myositis ossificans

4. Neoplasm of abdominal wall (incl. osteoma; osteosarcoma; chondrosarcoma)

5. [Opaque medication]

6. Parasitic disease (esp. guinea worm infection or cysticercosis of abdominal wall or back; Armillifer infection of peritoneum)

7. Scar; burn

8. Scleroderma; dermatomyositis

9. [Skin nodule; tattoo; colostomy orifice]

[ ] This condition does not actually cause the gamuted imaging finding, but can produce imaging changes that simulate it.

\section{References}

1. Baker SR, Cho KC: Abdominal calcifications. In Gore RM, Levine MS (eds): Textbook of Gastrointestinal Radiology. (ed 2) Philadelphia: WB Saunders, 2000, pp 178-189

2. Eisenberg RL: Gastrointestinal Radiology: A Pattern Approach. (ed 3) Philadelphia: Lippincott, 1996, pp 10491051 


\section{Gamut G-253}

\section{ABDOMINAL WALL MASS (ESP. ON US, CT, OR MRI)}

\section{COMMON}

1. Abscess $_{\mathrm{g}}$

2. Hematoma

3. Umbilical or ventral hernia

\section{UNCOMMON}

1. Endometrioma in scar

2. Neoplasm (eg, desmoid tumor; lipoma; neurofibroma; malignant fibrous histiocytoma; metastasis)

3. Omphalomesenteric cyst

4. Seroma; cellulitis

5. Urachal cyst

\section{References}

1. Marn CS: Anterior abdominal wall. In: Gore RM, Levine MS (eds): Textbook of Gastrointestinal Radiology. (ed 2) Philadelphia: WB Saunders, 2000, pp 2010-2019

2. Siegel MJ: Pediatric Body CT. Philadelphia: Lippincott Williams \& Wilkins, 1999

3. Williamson MR: Chapter 5. Abdominal ultrasound. In: Essentials of Ultrasound. Philadelphia: WB Saunders, 1996, p 68

\section{Gamut G-254}

\section{CYSTIC ABDOMINAL MASS IN A FETUS OR NEWBORN (US)}

\section{COMMON}
1. Dilated bladder
2. [Dilated bowel]
3. Hydronephrosis
4. Multicystic dysplastic kidney
5. Ovarian cyst

\section{UNCOMMON}

1. Choledochal cyst

2. Dilated ureter

3. Enteric duplication cyst

4. Hepatic cyst or cystic tumor (eg, mesenchymal hamartoma)

5. Hydrometrocolpos

6. Lymphangioma (incl. mesenteric cyst)

7. Persistent cloaca

8. Splenic cyst

9. Urachal cyst

10. Urinoma

[ ] This condition does not actually cause the gamuted imaging finding, but can produce imaging changes that simulate it.

\section{References}

1. Bryan, PJ: Lecture at Eastern Radiological Society Meeting, Dublin, Ireland, 1990.

2. Fernbach SK: Neonatal gastrointestinal radiology. In: Gore RM, Levine MS (eds): Textbook of Gastrointestinal Radiology. (ed 2) Philadelphia: WB Saunders, 2000, pp 2042-2073

\section{Gamut G-255}

\section{UPPER ABDOMINAL MASS IN A NEONATE OR CHILD}

\section{COMMON}

1. Abscess ${ }_{\mathrm{g}}$, abdominal (eg, retrocecal appendiceal)

2. Adrenal hemorrhage, cyst, or tumor (See H-124)

3. Fecal masses (esp. Hirschsprung disease)

4. Gastric dilatation (fluid filled stomach)

5. Hematoma (esp. splenic)

6. Hepatomegaly (See G-141-1 and -2)

7. Hydronephrosis

8. Intestinal obstruction (fluid-filled loop)

9. Intussusception

10. Lymphoma

11. Metastasis

12. Multicystic dysplastic kidney

13. Neuroblastoma; other neural tumor 
14. Pyloric stenosis

15. Renal neoplasm (eg, Wilms' tumor; mesoblastic nephroma) (See H-42)

16. Splenomegaly (See G-197, 198)

\section{UNCOMMON}

1. Bezoar, gastric

2. Bone lesion (eg, aneurysmal bone cyst or sarcoma of spine)

3. Cyst (eg, mesenteric; omental; renal; splenic; pancreatic; hepatic; choledochal; duplication; dermoid; hydatid)

4. Hepatic mass (eg, abscess; cyst; hepatoblastoma; hemangioma; hemangioendothelioma; hepatoma) (See G-154-157)

5. Infantile polycystic kidneys

6. Lymphangioma of mesentery or omentum

7. Meconium (eg, in cystic fibrosis)

8. Meningocele (anterior; lateral)

9. Renal vein thrombosis

10. Retroperitoneal lesion (eg, teratoma; sarcoma; hematoma)

11. Urinoma; lymphocele

\section{References}

1. Chan CY, Chan SM, Liauw L: A large abdominal mass in a young girl. Br J Radiol 2000;7:913-914

2. Fernbach SK: Neonatal gastrointestinal radiology. In: Gore RM, Levine MS (eds): Textbook of Gastrointestinal Radiology. (ed 2) Philadelphia: WB Saunders, 2000, pp 20422073

3. Kirks DR, Merten DF, Grossman H, Bowie JD: Diagnostic imaging of pediatric abdominal masses: an overview. Radiol Clin North Am 1981;19:527-545

4. Koop CE: Abdominal mass in the newborn infant. N Engl J Med 1973;289:569-571

5. Melicow MM, Uson AC: Palpable abdominal masses in infants and children: A report based on a review of 653 cases. J Urol 1959;81:705-710

6. Siegel MJ: Pediatric Body CT. Philadelphia: Lippincott Williams \& Wilkins, 1999

7. Swischuk LE, John SD: Differential Diagnosis in Pediatric Radiology. (ed 2) Baltimore: Williams \& Wilkins, 1995

8. Tank ES, Poznanski AK, Holt JF: The radiologic discrimination of abdominal masses in infants. J Urol 1973;109: $128-132$

9. Wedge JJ, Grosfeld JL, Smith JP: Abdominal masses in the newborn: 63 cases. J Urol 1971;106:770-775

\section{Gamut G-256}

\section{LOWER ABDOMINAL MASS IN A NEONATE OR CHILD}

\section{COMMON}

1. Abscess ${ }_{\mathrm{g}}$, abdominal (eg, appendiceal)

2. Bladder, distended

3. Fecal impaction and masses (esp. Hirschsprung disease)

4. Intestinal obstruction (fluid-filled loop)

5. Intussusception

6. Lymphoma

7. Metastasis

8. Ovarian cyst

9. Pregnancy

\section{UNCOMMON}

1. Bone lesion (eg, aneurysmal bone cyst or sarcoma of pelvis or LS spine)

2. Cyst, other (eg, mesenteric; dermoid; urachal; duplication; hydatid)

3. Hydrometrocolpos

4. Meconium (eg, in cystic fibrosis)

5. Meningocele (anterior; lateral)

6. Retroperitoneal lesion (eg, teratoma; sarcoma; hematoma)

7. Urinoma; lymphocele

\section{References}

1. Chan CY, Chan SM, Liauw L: A large abdominal mass in a young girl. Br J Radiol 2000; 7:913-914

2. Fernbach SK: Neonatal gastrointestinal radiology. In: Gore RM, Levine MS (eds): Textbook of Gastrointestinal Radiology. (ed 2) Philadelphia: WB Saunders, 2000, pp 20422073

3. Kirks DR, Merten DF, Grossman H, Bowie JD: Diagnostic imaging of pediatric abdominal masses: an overview. Radiol Clin North Am 1981;19:527-545

4. Koop CE: Abdominal mass in the newborn infant. N Engl J Med 1973;289:569-571

5. Melicow MM, Uson AC: Palpable abdominal masses in infants and children: A report based on a review of 653 cases. J Urol 1959;81:705-710 
6. Moss H, Wright DW, Weiss RG: Abdominal masses in pediatric patients. In: Wyllie R, Hyams JS: Pediatric Gastrointestinal Disease. (ed 2) Philadelphia: WB Saunders, 1999, pp 126-127

7. Siegel MJ: Pediatric Body CT. Philadelphia: Lippincott Williams \& Wilkins, 1999

8. Swischuk LE, John SD: Differential Diagnosis in Pediatric Radiology. (ed 2) Baltimore: Williams \& Wilkins, 1995

9. Tank ES, Poznanski AK, Holt JF: The radiologic discrimination of abdominal masses in infants. J Urol 1973;109: $128-132$

10. Wedge JJ, Grosfeld JL, Smith JP: Abdominal masses in the newborn: 63 cases. J Urol 1971;106:770-775

\section{Gamut G-257}

\section{ABDOMINAL LYMPHADENOPATHY (US, CT, MRI)}

\section{BENIGN}

+1 . AIDS (eg, bacillary angiomatosis); AIDS related complex

2. Amyloidosis

3. Castleman disease

4. Cavitary mesenteric lymph node syndrome

5. Cirrhosis (alcohol induced); primary biliary cirrhosis

6. Crohn's disease

7. Diverticulitis

*8. Mycobacterial infection (eg, Mycobacterium tuberculosis; M. avium intracellulare)

9. Pyogenic infection

10. Sarcoidosis

11. Sclerosing cholangitis

12. Whipple's disease

\section{MALIGNANT}

+1. Kaposi sarcoma

2. Lymphoma ${ }_{\mathrm{g}}$; Hodgkin disease; leukemia

*3. Metastatic disease

* Lymph nodes may show low attenuation.

+ Lymph nodes may show very high attenuation.

\section{References}

1. Chintapalli KN, Esola CC, Chopra S, et al: Pericolic mesenteric lymph nodes: an aid in distinguishing diverticulitis from cancer of the colon. AJR 1997;1253

2. Dodd GD, Baron RL, Oliver JH, et al: Enlarged abdominal lymph nodes in end-stage cirrhosis: CT-histopathologic correlation in 507 patients. Radiology 1997; 203:127-130

3. Dodd GD, Baron RL, Oliver JH, et al: Spectrum of imaging findings of the liver in endstage cirrhosis: part 1, gross morphology and diffuse abnormalities. AJR 1999;173:11851192

4. Okada Y, Yao YK, Yunoki M, et al: Lymph nodes in the hepatoduodenal ligament: US appearances with CT and MR correlation. Clin Radiol 1996;51:160-166

5. Warshauer DM, Dumbleton SA, Molina PL, et al: Abdominal CT findings in sarcoidosis: radiologic and clinical correlation. Radiology 1994;192:93-98

\section{Gamut G-258}

\section{FAT DENSITY IN THE ABDOMEN (CT, MRI)}

\section{COMMON}

1. Dermoid cyst; teratoma

2. Fatty change in liver or pancreas

3. Lipoma; liposarcoma

4. Lipomatosis (pelvic; retroperitoneal; renal sinus)

5. Obesity

\section{UNCOMMON}

1. Angiomyolipoma of kidney or liver

2. Omental or mesenteric hernia

3. Steroid therapy; Cushing syndrome

4. Xanthogranulomatous pyelonephritis

\section{Reference}

1. Katz DL: Fatty densities on CT. Semin Roentgenol 1978; 13:187 


\section{Gamut G-259}

\section{"PSEUDOKIDNEY" OR “BULL'S-EYE” SIGN IN THE ABDOMEN (US)}

\section{COMMON}

1. Gastroesophageal junction

2. Hypertrophic pyloric stenosis

3. Inflammatory bowel disease with wall thickening (eg, amebiasis; Crohn's disease; diverticulitis; Whipple's disease)

4. Intussusception (multiple concentric rings)

5. Malignant neoplasm with thickening of bowel wall

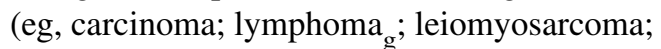
metastasis to serosa)

\section{UNCOMMON}

1. Amyloidosis

2. Intramural hematoma (eg, trauma; anticoagulants; blood dyscrasia ${ }_{\mathrm{g}}$ )

\section{Reference}

1. Eisenberg RL: Clinical Imaging: An Atlas of Differential Diagnosis. Gaithersburg, MD: Aspen Publ, 1992, pp 452-453

\section{Gamut G-260}

\section{ABNORMAL ABDOMINAL VESSELS ON ANGIOGRAPHY}

\section{COMMON}

1. Aneurysm

2. Anomalous origin or congenital absence of a vessel (eg, sequestration of lung)

3. Arterial occlusion, incl. collateral circulation (eg, via artery of Drummond; arc of Riolan; meandering mesenteric artery)

4. Arteritis, microaneurysms (eg, Behçet S.; Takayasu arteritis; necrotizing angiitis from drug abuse; my- cotic aneurysms; polyarteritis nodosa; other connective tissue disease $\{\text { collagen disease }\}_{\mathrm{g}}$ )

5. Atherosclerosis

6. Fibromuscular dysplasia

7. Neoplasm, incl. neovascularity (eg, angiomyolipoma; pheochromocytoma; renal cell carcinoma) or vascular cuffing or displacement (esp. carcinoma)

8. Thromboembolism

9. Trauma (lacerated or transected vessel)

10. Varices (eg, portal venous hypertension or obstruction; inferior vena cava obstruction)

\section{UNCOMMON}

1. Anomalous pulmonary vein draining below the diaphragm (incl. venolobar S.)

2. Arteriovenous communication

3. Azygos continuation of inferior vena cava

4. Coarctation of abdominal aorta

5. Neurofibromatosis, arterial

6. Phlebitis (esp. pylephlebitis)

7. Portal vein occlusion ("cavernous transformation")

8. Pregnancy (eg, hypertrophied uterine vessels; compression of iliac vein)

9. Pseudoxanthoma elasticum

10. Telangiectasia (eg, Osler-Weber-Rendu S.)

\section{References}

1. Moskowitz M, Zimmerman H, Felson B: The meandering mesenteric artery of the colon. AJR 1964;92:1088-1099

2. Nemcek AA, Vogelzang RL: Angiography and interventional radiology of the alimentary tract. In: Gore RM, Levine MS (eds): Textbook of Gastrointestinal Radiology. (ed 2) Philadelphia: WB Saunders, 2000, pp 509-511 


\section{Gamut G-261}

\section{COMPLICATIONS OF AIDS IN THE GASTROINTESTINAL TRACT AND ABDOMEN}

\section{ESOPHAGITIS}

1. Candidiasis (moniliasis)

2. Cytomegalovirus

3. Herpes

4. HIV

\section{GASTRITIS}

1. Cryptosporidium antritis

2. Cytomegalovirus infection (aphthoid ulcers; thick rugae, esp. at esophagogastric junction and antrum)

\section{ENTERITIS WITH THICK IRREGULAR FOLDS, SPASM, AND OCCASIONAL DILATATION}

1. Cryptosporidiosis

2. Cytomegalovirus infection

3. Mycobacterium avium-intracellulare infection

4. Parasitic disease (esp. giardiasis; strongyloidiasis)

\section{COLITIS}

1. Acute appendicitis

2. Cytomegalovirus infection of ileum and colon

3. Infectious colitis, esp. in rectum of homosexual men (eg, amebiasis; shigellosis; lymphogranuloma venereum \{chlamydial proctitis\}; gonorrhea)

4. Pseudomembranous colitis

\section{NEOPLASM OF GI TRACT, OCCASIONALLY WITH INTUSSUSCEPTION OR OBSTRUCTION}

1. Kaposi sarcoma

2. Lymphoma ${ }_{\mathrm{g}}$

\section{LIVER AND BILE DUCT INVOLVEMENT}

1. AIDS-related cholangitis (eg, cytomegalovirus;

Cryptosporidium)
2. Liver abscess, infection (eg, Mycobacterium aviumintracellulare; Pneumocystis carinii; cytomegalovirus; histoplasmosis; cryptococcosis; bacillary angiomatosis)

3. Neoplasm (incl. lymphoma)

4. Peliosis hepatis

\section{SPLENIC INVOLVEMENT}

1. Lymphoma

2. Splenic abscess, infection (eg, Mycobacterium avium-intracellulare; istoplasmosis; cryptococcosis; cytomegalovirus; Pneumocystis carinii)

3. Splenic infarcts (septic emboli)

4. Splenomegaly

\section{References}

1. Gore RM, Miller FH, Yaghmai V: Acquired immunodeficiency syndrome (AIDS) of the abdominal organs; imaging features. Semin US, CT, and MR 1998;19:175-189

2. Janoff EN, Smith PD: Emerging concepts in gastrointestinal aspects of HIV-1 pathogenesis and management. Gastroenterology 2001;120:607-621 